Alexander Pastwa

\title{
Serviceorientierung im betrieblichen Berichtswesen
}

Entwicklung eines Architektur- und Vorgehensmodells zur konzeptionellen Gestaltung von Berichtsprozessen auf Basis einer SOA und XBRL 


\section{Alexander Pastwa}

\section{Serviceorientierung im betrieblichen Berichtswesen}

Die Herausforderung, Entscheidungsträger mit „den richtigen Informationen zum richtigen Zeitpunkt in der richtigen Form" zu versorgen, legt nahe, die effiziente und effektive Verarbeitung der Berichtsinformationen aus den Perspektiven der Prozess- und Serviceorientierung zu betrachten. Damit sich Berichtsinformationen mit Hilfe von Berichtsservices zu einem Serviceorientierten Berichtsprozess zusammenfügen lassen, müssen die entsprechenden Dienste konzeptionell gestaltet und der resultierende Berichtsprozess in einem Architekturmodell eingebettet werden. Der Autor zeigt in dieser Arbeit, wie sich die innovativen Konzepte einer SOA und XBRL zu einem Architekturkonzept und Vorgehensmodell für die konzeptionelle Gestaltung Serviceorientierter Berichtsprozesse integrieren lassen.

Alexander Pastwa wurde 1976 in Kattowitz (Polen) geboren. Er schloss sein Studium der Wirtschaftswissenschaft an der Universität Bochum in 2003 mit dem Diplom ab. Im Herbst 2009 folgte die Promotion an der Fakultät für Wirtschaftswissenschaft. Während seiner Zeit als Wissenschaftlicher Mitarbeiter am Lehrstuhl für Wirtschaftsinformatik arbeitete er schwerpunktmäßig im Bereich Business Intelligence und war an verschiedenen Projekten und zahlreichen Veröffentlichungen zu diesem Themenfeld beteiligt. 
Serviceorientierung im betrieblichen Berichtswesen 


\section{BOCHUMER BEITRÄGE ZUR UNTERNEHMENSFÜHRUNG}

Herausgegeben von

Prof. Dr. Michael Abramovici, Prof. Dr. Dr. h.c. mult. Walther Busse von Colbe, Prof. Dr. Dr. h.c. Werner H. Engelhardt, Prof. Dr. Roland Gabriel, Prof. Dr. Wolfgang Maßberg, Prof. Dr. Horst Meier, Prof. Dr. Heiko Müller, Prof. Dr. Bernhard Pellens, Prof. Dr. Mario Rese, Prof. Dr. Marion Steven, Prof. Dr. Rolf Wartmann, Prof. Dr. Brigitte Werners

Band 79

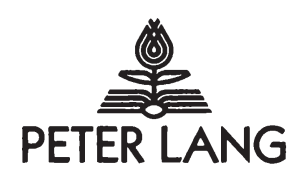

Frankfurt am Main - Berlin - Bern · Bruxelles - New York · Oxford - Wien 


\title{
Alexander Pastwa
}

\section{Serviceorientierung im betrieblichen Berichtswesen}

\author{
Entwicklung eines Architektur- und \\ Vorgehensmodells zur konzeptionellen \\ Gestaltung von Berichtsprozessen \\ auf Basis einer SOA und XBRL
}

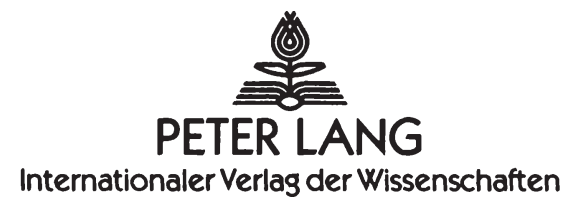


Bibliografische Information der Deutschen Nationalbibliothek Die Deutsche Nationalbibliothek verzeichnet diese Publikation in der Deutschen Nationalbibliografie; detaillierte bibliografische Daten sind im Internet über http://dnb.d-nb.de abrufbar.

Open Access: The online version of this publication is published on www.peterlang.com and www.econstor.eu under the international Creative Commons License CC-BY 4.0. Learn more on how you can use and share this work: http://creativecommons.org/ licenses/by/4.0.

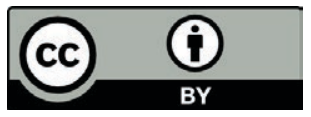

This book is available Open Access thanks to the kind support of ZBW - Leibniz-Informationszentrum Wirtschaft.

Zugl.: Bochum, Univ., Diss., 2009

Umschlaggestaltung: Atelier Platen, Friedberg

Gedruckt auf alterungsbeständigem, säurefreiem Papier.

\author{
D 294 \\ ISSN $1860-479 x$ \\ ISBN 978-3-631-60840-1 \\ ISBN 978-3-631-75488-7 (eBook) \\ (C) Peter Lang GmbH \\ Internationaler Verlag der Wissenschaften \\ Frankfurt am Main 2010 \\ Alle Rechte vorbehalten.
}

Das Werk einschließlich aller seiner Teile ist urheberrechtlich geschützt. Jede Verwertung außerhalb der engen Grenzen des Urheberrechtsgesetzes ist ohne Zustimmung des Verlages unzulässig und strafbar. Das gilt insbesondere für Vervielfältigungen, Übersetzungen, Mikroverfilmungen und die Einspeicherung und Verarbeitung in elektronischen Systemen.

www.peterlang.de 
Meinen Eltern.

Und meinen Großeltern, die dieses

Buch gerne in Händen gehalten hätten. 
Alexander Pastwa - 978-3-631-75488-7

Downloaded from PubFactory at 01/11/2019 04:20:29AM

via free access 


\section{Geleitwort}

Trotz seiner Bedeutung als Instrument zur Unternehmenssteuerung und der Existenz vieler Beiträge, die sich mit der Gestaltung und dem Betrieb von Informationssystemen (IS) auseinandersetzen, sind das interne und externe ISgestützte Reporting nach wie vor mit diversen Problemfeldern konfrontiert, die ein aussagekräftiges und verlässliches Berichtswesen beeinträchtigen. Die Störungsursachen treten infolge einer mangelnden Integration der zum Einsatz kommenden Berichtsdaten, -funktionen, -prozesse und -systeme auf. Aus der Perspektive der Wirtschaftsinformatik interessieren in diesem Zusammenhang neben den Einsatzmöglichkeiten innovativer und leistungsfähiger Informationstechnik (IT) auch die Gestaltbarkeit und Umsetzbarkeit geeigneter Prozesse zur Unterstützung der Berichtszwecke. Fragestellungen, die sich mit dem gezielten Einsatz von IT zur Informationsversorgung von Entscheidungsträgern sowie zur Unterstützung von Geschäftsprozessen beschäftigen, sind eine zentrale Säule in der Forschung des Lehrstuhls für Wirtschaftsinformatik an der Ruhr-Universität Bochum. Eine Reihe von wissenschaftlichen Beiträgen wurde zu diesem Themengebiet in den vergangenen Jahren veröffentlicht.

Die Herausforderung, Entscheidungsträger auf allen Hierarchieebenen einer Unternehmungsorganisation mit ,den richtigen Informationen zum richtigen Zeitpunkt in der richtigen Form" zu versorgen, legt nahe, die effiziente und effektive Verarbeitung der Berichtsinformationen nicht nur aus der Perspektive der Prozessorientierung zu betrachten. Bei der berichtsempfängerorientierten Erstellung, Ablage und Diffusion der Reports spielt auch zunehmend der Dienst- bzw. Servicegedanke eine wichtige Rolle. Damit sich Berichtsinformationen mit Hilfe derartiger „Berichtsservices“ zu einem Serviceorientierten Berichtsprozess zusammenfügen lassen, müssen die entsprechenden Dienste konzeptionell gestaltet und der resultierende Berichtsprozess in einem Architekturmodell eingebettet werden.

Innovative Konzepte, die aus der Perspektive der Wirtschaftsinformatik zur Unterstützung Serviceorientierter Berichtsprozesse in Frage kommen, werden aktuell unter den Begriffen Serviceorientierte Architektur (SOA) sowie Extensible Business Reporting Language (XBRL) diskutiert. Herrn Pastwa gelingt es in der vorliegenden Dissertationsschrift in ausgezeichneter Form, sowohl SOA als auch XBRL, die bis auf wenige Publikationen bisher isoliert voneinander betrachtet wurden, zu einem Architekturkonzept und Vorgehensmodell zur konzeptionellen Gestaltung Serviceorientierter Berichtsprozesse zu integrieren. 
Der Autor leistet mit seiner Arbeit einen wesentlichen Fortschritt zur Lösung des gegebenen Problems. Ich wünsche dem vorliegenden Buch eine gute Aufnahme in die weitere wissenschaftliche Diskussion, die auch im betrieblichen Rechnungswesen immer intensiver geführt wird.

Bochum, im Mai 2010

Roland Gabriel 


\section{Dankeswort}

Die vorliegende Arbeit habe ich im Rahmen meiner Tätigkeit als wissenschaftlicher Mitarbeiter am Lehrstuhl für Wirtschaftsinformatik von Professor Dr. Roland Gabriel angefertigt. Im Oktober 2009 wurde sie von der Fakultät für Wirtschaftswissenschaft der Ruhr-Universität Bochum als Dissertationsschrift angenommen.

Bekanntlich tut es immer gut, mit Rückenwind einen längeren Weg zu beschreiten. Das Verfassen einer Dissertationsschrift kommt einer Reise gleich, deren Ziel feststeht und deren Weg zu meistern, eine große Herausforderung darstellt. Viele Menschen haben mir reichlich Rückenwind geschenkt und mir damit geholfen, ans Ziel zu gelangen. Diesen Menschen spreche ich für die vielen Momente der Unterstützung meinen tiefen Dank aus. Ihnen gebühren diese Zeilen.

Allen voran danke ich meinem Doktorvater Herrn Professor Dr. Roland Gabriel. Ich danke Ihnen, Herr Gabriel, für Ihre fachlichen Anregungen und wissenschaftlichen Diskussionen, für Ihre engagierte Unterstützung sowie die mir eingeräumte Freiheit, mich mit diesem Thema in meiner Dissertationsschrift beschäftigen zu können. Für die Übernahme des Zweitgutachtens und die vielen wertvollen Ratschläge danke ich Frau Professor Dr. Brigitte Werners.

Ein besonderer Dank gilt meinem Büronachbarn Diplom-Ökonom Tobias Hoppe, der stets ein offenes Ohr für meine Fragen hatte. Lieber Tobias, ich danke Dir für den stets produktiven wissenschaftlichen Austausch. Mit deinem kompetenten und ehrlichen Urteil hast du maßgeblich zum Gelingen meiner Arbeit beigetragen. $\mathrm{Zu}$ Dank bin ich auch Dr. Sebastian Sowa verpflichtet, der mich als Ideengeber während der Realisierung meiner Dissertation in unschätzbarer Weise unterstützt hat.

Danken möchte ich Dr. Carsten Dittmar, der mich seinerzeit dazu motiviert hat, mich als wissenschaftlicher Mitarbeiter zu bewerben. Durch seine Begeisterung für das Thema Business Intelligence wurde auch in mir der Ansporn geweckt, mich wissenschaftlich mit diesem Thema auseinanderzusetzen. Ebenso danke ich Professor Dr. Peter Gluchowski, der nie das Interesse am Thema und am Fortschritt meiner Arbeit verloren hat und dessen Anregungen sowie Gespräche stets eine Bereicherung waren.

$\mathrm{Zu}$ Dank bin ich Dr. Thomas Lux sowie Dr. Roland Düsing für die vielen konstruktiven Gespräche bei der Themenfindung sowie Weiterentwicklung meiner Arbeit verpflichtet. Ein Dankeswort möchte ich auch an die Sekretärin des Lehrstuhls Frau Susanne Schutta richten, die in ihrer freundlichen und hilfsbereiten Art mir immer zur Seite stand. 
Ferner möchte ich allen meinen wissenschaftlichen Kolleginnen und Kollegen sowie allen studentischen Hilfskräften für die produktive, lehrreiche und nie langweilige Zusammenarbeit danken.

$\mathrm{Zu}$ guter letzt gebührt besonderer Dank meinen Eltern Ursula und Christian Pastwa. Es bereitet mir eine große Freude, ihnen mit dieser Arbeit für ihre $\mathrm{Zu}$ neigung, ihren Glauben an mich sowie ihr tagtägliches Vorleben, niemals das Ziel vor den Augen zu verlieren, ein klein wenig zurückzugeben.

Dortmund, im Mai 2010

Alexander Pastwa 


\section{Inhaltsverzeichnis}

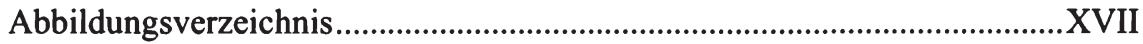

Abkürzungsverzeichnis .........................................................................

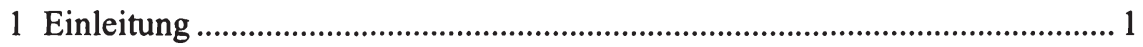

1.1 Problemstellung und Motivation der Arbeit ............................................. 1

1.2 Ziel und Gang der Untersuchung ............................................................. 2

2 Prozessunterstützung im betrieblichen Berichtswesen.................................... 7

2.1 Terminologische und konzeptionelle Grundlagen zum IT-gestützten Berichtswesen ......................................................................................... 10

2.1.1 Auslegungen des Berichtswesens.................................................... 11

2.1.2 Abgrenzung des Berichtswesens .................................................... 13

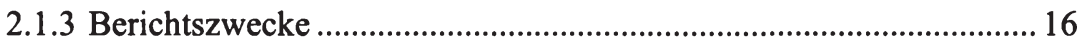

2.2 Berichtssysteme zur informationstechnischen Unterstützung von Berichtsprozessen ..................................................................................... 18

2.2.1 Berichtssysteme als Anwendungsbereich von Business Intelligence 18

2.2.1.1 Business Intelligence-Systeme (BI-Systeme) als übergeordnete Systemkategorie zur Gestaltung und Einordnung von Berichtsanwendungen ................................................ 18

2.2.1.2 Ausprägungen von Berichtssystemen .................................... 22

2.2.2 Kernprozesse des betrieblichen Berichtswesens ................................. 26

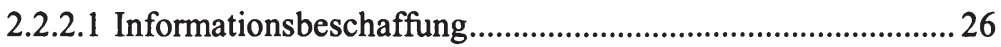

2.2.2.2 Berichtserstellung .................................................................... 27

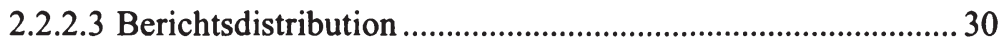

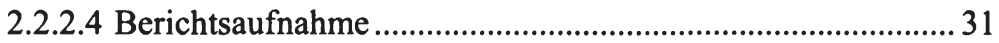


2.3 Defizite in den Kernprozessen des Berichtswesens................................... 33

2.3.1 Zweckbezogene Defizite für die Prozesse „Berichtsdistribution“ und ,Berichtsaufnahme““............................................................... 35

2.3.1.1 Geringe Empfängerorientierung............................................ 35

2.3.1.2 Niedrige Informationsaktualität .......................................... 39

2.3.2 Inhaltsbezogene Defizite für die Prozesse „Berichtserstellung“ und „Berichtsaufnahme“.

2.3.3 Syntaxbezogene Defizite für alle Teilprozesse des Berichtswesens.. 43

2.3.3.1 Technisch bedingte Störungen .............................................. 44

2.3.3.2 Personen bedingte Störungen................................................. 47

2.4 Zusammenfassung und kritische Würdigung........................................... 50

3 Serviceorientierte Architekturen (SOA) als innovatives Konzept für eine integrierte Informationsverarbeitung ............................................................53

3.1 Integrierte Informationsverarbeitung im Kontext aktueller SOA-Treiber... 55

3.1.1 Begriff der integrierten Informationsverarbeitung ............................. 55

3.1.2 Betriebliche Anwendungssysteme - State of the Art........................58

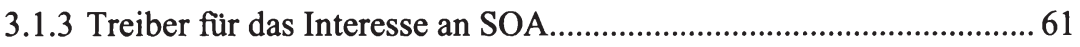

3.1.3.1 Kosten des IT-Betriebs........................................................ 62

3.1.3.2 Mangelnde IT-Unterstützung von Geschäftsprozessen ......... 68

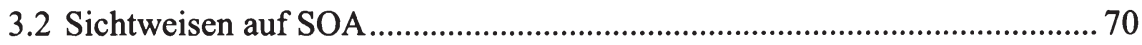

3.2.1 SOA als Informationssystem-Architektur ..................................... 72

3.2.2 SOA als konkrete technische Implementierung eines Softwaresystems auf Basis von Web Services.................................................. 75

3.2.3 SOA als Konzept zur Gestaltung prozessorientierter Anwendungen 76

3.3 Services als Basiselemente einer SOA ……............................................... 78

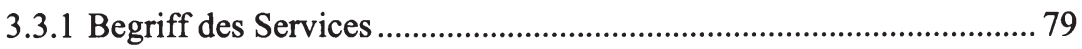


3.3.2 Prinzipien zum Design von Services 80

3.3.2.1 Prinzip der „Abstraktion von der Implementierungslogik“... 81

3.3.2.2 Prinzipien der „hohen Servicekohäsion“ und der „schwachen logischen Kopplung“

3.3.2.3 Prinzip der „Verwendung technischer und offener Industriestandards"

3.3.3 Rollenkonzept einer SOA 85

3.4 Web Services zur Implementierung Serviceorientierter Anwendungen...... 87

3.4.1 Grundverständnis zum Begriff des Web Services............................... 88

3.4.2 Bausteine einer Web Serviceorientierten Anwendung ......................89

3.4.2.1 Nachrichtenaustausch mit SOAP .........................................90

3.4.2.2 Schnittstellendefinition mit WSDL........................................ 93

3.4.2.3 Veröffentlichung mit UDDI ................................................ 95

3.5 Zusammenfassung und Ableitung eines integrierten SOA-Verständnisses 97

4 Architekturmodell für Serviceorientierte Berichtsprozesse ........................ 105

4.1 Begriff eines Serviceorientierten Berichtsprozesses................................. 106

4.2 SOA-Ebenenmodell als geeignetes Architekturkonzept für Serviceorientierte Berichtsprozesse

4.2.1 Ebene der Anwendungssysteme ................................................... 108

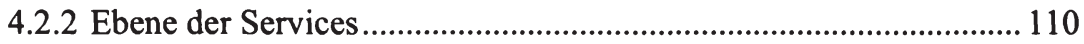

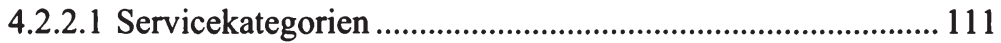

4.2.2.2 Middlewaredienste .......................................................... 113

4.2.2.3 Beziehungen zwischen den Komponenten der

Serviceebene ..................................................................... 114

4.2.3 Ebene der Prozesse ........................................................................... 115

4.2.3.1 Worflow Management-Systeme........................................... 115 
4.2.3.2 Kopplungsmechanismen zwischen Services........................ 118

4.2.3.3 Beziehungen zwischen den Komponenten der Prozessebene.

4.2.4 Ebene der Frontendsysteme

4.2.5 Eignung des SOA-Ebenenmodells für Serviceorientierte Berichtsprozesse

4.3 Erweiterung des SOA-Architekturmodells um eine XBRL-Ebene 125

4.3.1 Extensible Business Reporting Language (XBRL) als Enabler eines Serviceorientierten Berichtsprozesses.

4.3.1.1 XML zur semantischen Beschreibung von Informationen.. 125

4.3.1.2 Definition von XBRL 128

4.3.2 Bausteine des XBRL-Standards 129

4.3.2.1 XBRL-Taxonomie

4.3.2.1.1 XBRL-Schema als Normierungs- und Strukturvorschrift einer XBRL-Taxonomie. 130

4.3.2.1.2 Beziehungstypen einer Taxonomie 132

4.3.2.2 Taxonomiekategorien von XBRL. 133

4.3.2.2.1 XBRL Financial Reporting 133

4.3.2.2.2 XBRL Global Ledger 134

4.3.2.3 Werttragende Instanzdokumente.

4.3.3 Einbindung der XBRL-Konzepte in das SOA-Ebenenmodell

4.4 Zusammenfassung und Würdigung des um XBRL erweiterten SOAArchitekturmodells

5 Vorgehensmodell zur konzeptionellen Gestaltung Serviceorientierter Berichtsprozesse

5.1 Evaluation bestehender Vorgehensstrategien zur Gestaltung

Serviceorientierter Anwendungen

5.1.1 Top-down-Ansatz

5.1.2 Bottom-up-Ansatz 146 
5.1.3 Meet-in-the-middle-Ansatz

5.1.4 Bewertung der bestehenden Ansätze für die konzeptionelle Gestaltung Serviceorientierter Berichtsprozesse.

5.2 Konzeptionelle Gestaltung Serviceorientierter Berichtsprozesse.

5.2.1 Berichtsprozessanalyse aus der Perspektive der Serviceorientierung

5.2.1.1 Zielbestimmung der zu gestaltenden Serviceorientierten Berichtsprozesse anhand eines Ordnungsrahmens

5.2.1.2 Modellierungsmethodengestützte Erstellung neuer Berichtsprozesse oder Veränderung bestehender Berichtsprozesse

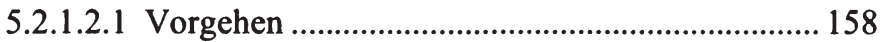

5.2.1.2.2 Einsatz von Modellierungsmethoden................. 160

5.2.1.3 Einbindung einer Lebenszyklusbetrachtung der

Berichtsservices als übergeordneter Prozess

5.2.2 Prozessmodellbasierte Serviceidentifikation. 167

5.2.2.1 Wiederverwendung als Ziel der prozessmodellbasierten

Serviceidentifikation 167

5.2.2.2 Top-down-gestützte Serviceidentifikation 169

5.2.2.2.1 Vorgehen

5.2.2.2.2 Beispiele für Berichtsservicekandidaten

5.2.2.2.2.1 Berechnung verknüpfter Kennzahlen im Rahmen des Prozesses „Berichtserstellung“.

5.2.2.2.2.2 XBRL-Validierung von

Jahresabschlussdaten im Rahmen

der Prozesse „Informations-

beschaffung“" und „Berichts-

distribution" 174

5.2.2.3 Bottom-up-gestützte Serviceidentifikation

5.2.3 Fachliche Servicegestaltung 176

5.2.3.1 Anforderungen zur fachlichen Servicegestaltung 177

5.2.3.2 Kategorisierung der potenziellen Berichtsservices 179 
5.2.3.3 Zuweisung von XBRL-Taxonomieelementen 180

5.2.3.4 Bewertung der Dienstgüte bereits vorhandener interner oder externer Berichtsservices

5.2.3.4.1 Qualitätskriterien zur Bewertung der Berichtsservices.

5.2.3.4.2 Vorgehen zur Bewertung der Dienstgüte. 184

5.2.3.5 Fachliche Gestaltung neuer Berichtsservices 186

5.3 Zusammenfassung und Würdigung des Vorgehensmodells 191

6 Zusammenfassung und Resümee 195

6.1 Allgemeine Zusammenfassung.

6.2 Zusammenfassung der Nutzenpotenziale des Architektur- und Vorgehensmodells zur Reduzierung der Defizite beim Reporting

6.2.1 Nutzenpotenziale zur Vermeidung zweckbezogener Defizite 197

6.2.2 Nutzenpotenziale zur Minderung inhaltsbezogener Defizite 198

6.2.3 Nutzenpotenziale zur Reduzierung syntaxbezogener Defizite 199

6.3 Ausblick und weiterer Forschungsbedarf. 202

Literaturverzeichnis XXIII 


\section{Abbildungsverzeichnis}

Abbildung 1: Grobgliederung der Untersuchung....................................................... 4

Abbildung 2: Einordnung des Berichtswesens nach Göpfert ................................. 12

Abbildung 3: Abgrenzung der Begriffe Berichtswesen, Unternehmenspublizität und Rechnungslegung.............................. 14

Abbildung 4: Aufbau eines BI-Systems ………………………………………..... 20

Abbildung 5: Übersicht zur Einordnung von Berichtssystemen.......................... 23

Abbildung 6: Phasen des Berichtsprozesses............................................................... 26

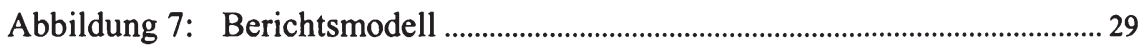

Abbildung 8: Übersicht zu den Problemfeldern bei der Informationsverarbeitung im betrieblichen Berichtswesen........ 35

Abbildung 9: Heterogenität der Datenstrukturen und Zugriffs-, Präsentations- sowie Datenaustauschformate .................................. 45

Abbildung 10: Ausgewählte Integrationsdimensionen ............................................ 57

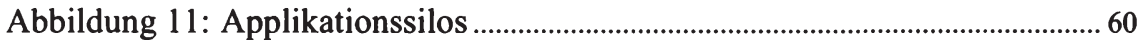

Abbildung 12: Literaturauswertung zu den Treibern einer SOA ........................... 62

Abbildung 13: Steigende Wartungskosten ...................................................................... 64

Abbildung 14: Zusammenhang zwischen Prozessaktivitäten, Services und Anwendungs systemen ............................................................................ 77

Abbildung 15: Servicemodell ................................................................................ 79

Abbildung 16: Aufruf eines Services ....................................................................... 82

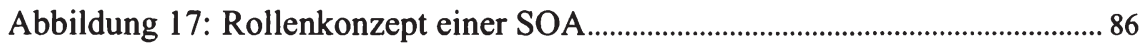

Abbildung 18: Aufruf eines Web Services............................................................ 91

Abbildung 19: Struktur einer SOAP-Nachricht .......................................................93

Abbildung 20: Aufbau eines WSDL-Dokuments....................................................... 94

Abbildung 21: Erweiterung der Integrationstypen um die Ebene der Serviceintegration.............................................................................. 100

Abbildung 22: Ebene der Anwendungssysteme ........................................................ 109

Abbildung 23: Erweiterung des SOA-Architekturmodells um die Ebene der Services . 
Abbildung 25: Zusammenhang zwischen Serviceorchestrierung und -choreografie

Abbildung 26: Erweiterung des SOA-Architekturmodells um die Prozessebene

Abbildung 27: Erweiterung des SOA-Architekturmodells um die Ebene der Frontendsysteme

Abbildung 28: Beispiel für ein XML-Dokument 128

Abbildung 29: Ausgewählte Attribute eines XBRL-Taxonomieelements. 131

Abbildung 30: Aufbau von XBRL GL 136

Abbildung 31: Beispiel eines XBRL-Instanzdokuments 137

Abbildung 32: Nutzungsmöglichen von XBRL 138

Abbildung 33: Erweiterung des SOA-Architekturmodells um die Ebene der Services 140

Abbildung 34: Top-down-Ansatz zur Einführung einer SOA 144

Abbildung 35: Vorgehen beim Top-down-Ansatz 146

Abbildung 36: Vorgehen beim Bottom-up-Ansatz 147

Abbildung 37: Meet-in-the-middle-Ansatz 150

Abbildung 38: Dimensionen zur Zielbestimmung eines SOBP 154

Abbildung 39: Exemplarische Zerlegung des Berichtsprozesses Informationsbeschaffung. 159

Abbildung 40: Lebenszyklus eines Berichtsservices 163

Abbildung 41: Beispiel eines aufbereiteten Aktivitätsdiagramms 170

Abbildung 42: ROI-Baum nach dem DuPont-Schema 173

Abbildung 43: Übersicht der Qualitätskriterien zur Beurteilung der Dienstgüte. 182

Abbildung 44: Beispiel einer Bewertungsmatrix 185 Abbildung 45: Übersicht der Schritte 1 bis 3 bei der fachlichen Servicegestaltung. 190 


\section{Abkürzungsverzeichnis}

$\begin{array}{ll}\text { AES } & \text { Advanced Encryption Standard } \\ \text { API } & \text { Application Programming Interface } \\ \text { BI } & \text { Business Intelligence } \\ \text { BPD } & \text { Business Process Diagram } \\ \text { BPEL } & \text { Business Process Execution Language } \\ \text { BPMN } & \text { Business Process Modeling Notation } \\ \text { CA } & \text { Composite Application }\end{array}$

CEBS Committee of European Banking Supervisors

CNMV Comisión Nacional del Mercado de Valores

COR Core

CORBA Common Object Request Broker Architecture

CRM Customer Relationship Management

CRUD Cread, Read, Update, Delete

CSS Cascading Style Sheets

CSV Comma Separated Values

DBS Datenbanksysteme

DCOM Distributed Component Object Model

DDL Data Definition Language

DML Data Manipulation Language

DMS Dokumentenmanagementsystem

DUNS Data Universal Numbering System

DWH Data Warehouse

eEPK Erweiterte Ereignisgesteuerte Prozesskette

EPK Ereignisgesteuerte Prozesskette

ER Entity Relationship

ERP Enterprise Resource Planning

ESB Enterprise Service Bus

ETL Extraktion, Transformation, Laden 
FR

Financial Reporting

FTP

File Transfer Protocol

GI

Gesellschaft für Informatik

GL

Global Ledger

GUI

Graphical User Interface

$\mathrm{GuV}$

Gewinn und Verlust

HGB

Handelsgesetzbuch

HTML

Hypertext Markup Language

HTTP

Hypertext Transfer Protocol

IAS

International Accounting Standards

IFRS

International Financial Reporting Standards

IS

Informationssystem

ISO

International Organisation for Standardization

IT

Informationstechnik

IuK

Information und Kommunikation

KMU

Kleine und mittlere Unternehmungen

KPI

Key Performance Indikator

MDB

Microsoft Database

MQE

Managed Query Environments

NAICS

North American Industry Classification System

OASIS

Organization for the Advancement of Structured Information Standards

ODBC Open Database Connectivity

OLAP

Online Analytical Processing

OLTP

Online Transaction Processing

PDF

Portable Document Format

QoS

Quality of Service

RMI

Remote Method Invocation

ROI

Return on Investment

RPC

Remote Procedure Call

SCM

Supply Chain Management 
sEPK Serviceorientierte Ereignisgesteuerte Prozesskette

SGML Standard Generalized Markup Language

SLA Service Level Agreement

SLO Service Level Object

SMTP Simple Mail Transfer Protocol

SOA Serviceorientierte Architektur

SOBP Serviceorientierter Berichtsprozess

SOPC Serviceorientiertes Prozesscontrolling

SQL Structured Query Language

SSE Shanghai Stock Exchange

TCP Transmission Control Protocol

UDDI Universal Description, Discovery and Integration

UNSPSC Universal Standard Products and Services Codes

US-GAAP United States Generally Accepted Accounting Principles

W3C World Wide Web Consortium

WES Workflow Enactment Service

WMS Workflow Management-System

WSCI Web Service Choreography Interface

WSDL Web Services Description Language

WSFL Web Services Flow Language

WWW World Wide Web

XBRL Extensible Business Reporting Language

XLS Excel Spreadsheet

XML Extensible Markup Language

XSL Extensible Stylesheet Language 
Alexander Pastwa - 978-3-631-75488-7

Downloaded from PubFactory at 01/11/2019 04:20:29AM

via free access 


\section{Einleitung}

Abschnitt 1.1 führt in die Problemstellung und die Motivation der Arbeit ein. Daraufhin werden in Abschnitt 1.2 die Ziele der Arbeit sowie der Gang der Untersuchung vorgestellt.

\subsection{Problemstellung und Motivation der Arbeit}

Das betriebliche Berichtswesen bzw. das Unternehmensreporting gilt heute als wichtiges Instrument zur Dokumentation von Ereignissen, zur Auslösung von Aktivitäten sowie zur Vorbereitung und Kontrolle von Entscheidungen. Die effiziente und effektive Verarbeitung von Geschäfts- und Finanzinformationen hängt nicht nur von der Leistungsfähigkeit der eingesetzten Informationstechnik (IT) ab. Neben dem Einsatz IT-gestützter Berichtssysteme liegt ein weiterer wichtiger Faktor für ein zuverlässiges Reporting in der Gestaltbarkeit und dem erfolgreichen Betrieb geeigneter Berichtsprozesse. Die Kernprozesse innerhalb eines Berichtsprozesses umfassen die Aufnahme der in den Berichten bzw. Reports enthaltenen Informationen, die Produktion der Berichte, die Weiterleitung der Reports an den jeweiligen Berichtsempfänger(-kreis) und die Aufnahme der Berichte.

Trotz seiner Bedeutung als Instrument zur Unternehmenssteuerung und der Existenz vieler Arbeiten, die sich mit der Gestaltung und dem Betrieb ITgestützter Berichtsprozesse auseinandersetzen, ist das Unternehmensreporting nach wie vor mit einer Reihe von Problemfeldern konfrontiert, die eine effektive und effiziente Ausführung der Berichtsprozesse beeinträchtigen. Während die Effektivität des betrieblichen Berichtswesens durch eine mangelnde Empfängerorientierung, eine geringe Informationsaktualität sowie eine unzureichende semantische Verarbeitung der berichteten Informationen gefährdet ist, ergeben sich Ineffizienzen infolge technisch- und personenbedingter Störungen. Im Ergebnis ist zu konstatieren, dass die Störungsursachen Folge einer mangelnden Integration der zum Einsatz kommenden Berichtsdaten, -funktionen, -prozesse und -anwendungen sind.

Einen Ausweg zur Reduzierung dieser Störungsursachen bietet der derzeit intensiv diskutierte Ansatz einer "Serviceorientierten Architektur“ (SOA), die auch als „Dienstorientierte Architektur" bzw. „Service Oriented Architecture“ bezeichnet wird. SOA beschäftigt sich mit der Frage, auf welche Weise Anwendungssysteme gestaltet, integriert und wirtschaftlich betrieben werden sollen. Aus der Perspektive der Wirtschaftsinformatik richtet sich die Erwartungshaltung an SOA auf die Unterstützung einer integrierten Informationsverarbeitung mit dem Ziel, eine stärkere Flexibilität und/oder Automatisierung der Ge- 
schäftsprozesse durch den Einsatz wiederverwendbarer (Software-)Services zu erzielen. In den aktuellen Beiträgen, die sich mit dem Themenfeld SOA auseinandersetzen, erfolgt insbesondere eine Diskussion der zum Einsatz kommenden Prinzipien, die sich für die Gestaltung Serviceorientierter Anwendungen nutzen lassen.

\subsection{Ziel und Gang der Untersuchung}

Obwohl in den vergangenen Jahren viele Beiträge zum Konzept einer SOA entstanden sind, ist festzustellen, dass die Grundlagenarbeit noch nicht abgeschlossen ist. Aus einer methodischen Perspektive betrachtet, liegt, neben der Notwendigkeit, ein einheitliches Begriffsverständnis zu etablieren, ein wichtiges Forschungsfeld darin, eine geeignete Methodik für die Gestaltung einer SOA im Allgemeinen sowie zur Bereitstellung geeigneter Services zur Integration von Produktion und Dienstleistung im Besonderen zu entwickeln und einzusetzen. ${ }^{1}$ In diesem Zusammenhang sind geeignete Vorgehensweisen zur Spezifizierung sowie Verknüpfung von Diensten zu erarbeiten. ${ }^{2}$ Ein weiteres wichtiges Forschungsfeld richtet sich auf die Definition einer geeigneten SOA-Referenzarchitektur, ${ }^{3}$ die den strukturellen Bezugsrahmen zur Gestaltung Serviceorientierter Anwendungen liefert.

Damit sich Berichtsinformationen mit Hilfe von Berichtsservices entlang einer Prozesskette verarbeiten lassen, ist es erforderlich, dass die entsprechenden Berichtsprozesse modelliert und die zum Einsatz kommenden Services konzeptionell gestaltet werden. Derartige Services werden im Folgenden als Berichtsservices bezeichnet, während für einen Berichtsprozess, der mit Hilfe verknüpfter Services ausgeführt wird, der Terminus Serviceorientierter Berichtsprozess (SOBP) verwendet wird. Die konzeptionelle Gestaltung der in einem SOBP zum Einsatz kommenden Berichtsservices besitzt eine Bedeutung, da nur wiederverwendbare Services einen Mehrwert für die SOA-betreibende Unternehmung schaffen. Da SOA u. a. das Ziel verfolgt, bei der Ausführung eines Prozesses Services zu nutzen, die von unternehmungsexternen Anbietern zur Verfügung gestellt werden, besitzt die Anforderung, wiederverwendbare Services zu konzipieren, eine umso wichtigere Bedeutung.

Vor dem Hintergrund des Untersuchungsgegenstands dieser Arbeit wird die Frage erörtert, wie sich Serviceorientierte Konzepte für die effiziente und effektive Verarbeitung von Geschäfts- und Finanzinformationen nutzen lassen, um die im Zusammenhang mit der Ausführung von Berichtsprozessen auftretenden Problemfelder zu reduzieren. Damit wird der Versuch unternommen, ein be-

1 Vgl. Höß et al. (2007), S. 46; Beverungen/Knackstedt/Müller (2008), S. 221.

2 Vgl. Eymann/Winter (2008), S. 70.

3 Vgl. Eymann/Winter (2008), S. 70. 
triebswirtschaftliches Anwendungsfeld mit einem in der Wissenschaft aktuell diskutierten Architekturkonzept zu verbinden.

Mit Blick auf den Untersuchungsgegenstand dieser Arbeit, der die konzeptionelle Gestaltung von Berichtsservices fokussiert, ist zu bemerken, dass auf Aspekte, die die technische Umsetzung eines SOBP betreffen, nicht eingegangen wird. Die Eingrenzung auf die konzeptionelle Gestaltung von Berichtsprozessen erfolgt vor dem Hintergrund, dass die Implementierung von Services derzeit keinen Forschungsschwerpunkt bei der Entwicklung Serviceorientierter Anwendungen darstellt. Da es in den vergangenen Jahren gelungen ist, Standards sowie Software-Werkzeuge zur Realisierung Serviceorientierter Prozesse $\mathrm{zu}$ entwickeln, die sich in der Industrie etabliert haben, wird in dieser Arbeit auf die Auseinandersetzung mit diesem Aspekt verzichtet. Stattdessen ist in der wissenschaftlichen Auseinandersetzung mit SOA nach wie vor das Defizit zu konstatieren, dass eine methodische Herangehensweise zum Aufbau einer SOA fehlt.

Abbildung 1 stellt den Gang der Untersuchung auf Hauptkapitelebene dar. Das Ziel des Kapitels 2 liegt darin, unter Einbezug aktueller Erkenntnisse, eine Zustandsbeschreibung der Prozessunterstützung im betrieblichen Berichtswesen zu präsentieren. Der Fokus liegt zum einen auf der Erarbeitung begrifflicher, konzeptioneller und informationstechnischer Grundlagen des Berichtswesens. Im Mittelpunkt stehen dabei die Kernprozesse des Berichtswesens. Zum anderen werden in diesem Kapitel Problemfelder thematisiert, mit denen das Berichtswesen aktuell konfrontiert ist. Die Problemfelder betreffen sowohl die Effektivität als auch die Effizienz der Informationsverarbeitung und lassen sich aus der Perspektive der Semiotik betrachten.

Während heute in vielen Unternehmungen Ausprägungen von Business Intelligence-Systemen zur Unterstützung von Berichtsprozessen genutzt werden, steht mit dem Konzept einer SOA ein Ansatz zur Verfügung, der sich ebenfalls für die Zwecke des Reportings nutzen lässt. Der derzeitige Erkenntnisstand um SOA ist durch eine Vielzahl von Vorschlägen gekennzeichnet, die darauf fokussieren, den Gegenstandsbereich einer SOA zu definieren und Nutzungsmöglichkeiten dieses Konzepts aufzuzeigen. Das Ziel des Kapitels 3 liegt darin, ausgehend von aktuell zu beobachtenden Problemfeldern im IT-Betrieb und vier verschiedenen Auslegungen, die sich in den Beiträgen zum SOA-Konzept identifizieren lassen, ein integriertes SOA-Verständnis zu präsentieren. Auch wenn die Auslegungen verschiedene Facetten einer SOA betonen, zeichnen sie sich durch die Gemeinsamkeit aus, dass die Serviceorientierung den Leitgedanken einer SOA darstellt. Daher wird im Folgenden auch die Frage erörtert, welche grundlegenden Eigenschaften und Gestaltungsprinzipien einen Service auszeichnen. Mit den Web Services wird daraufhin eine Technologie vorgestellt, die zur Implementierung von Services zum Einsatz kommen kann. 


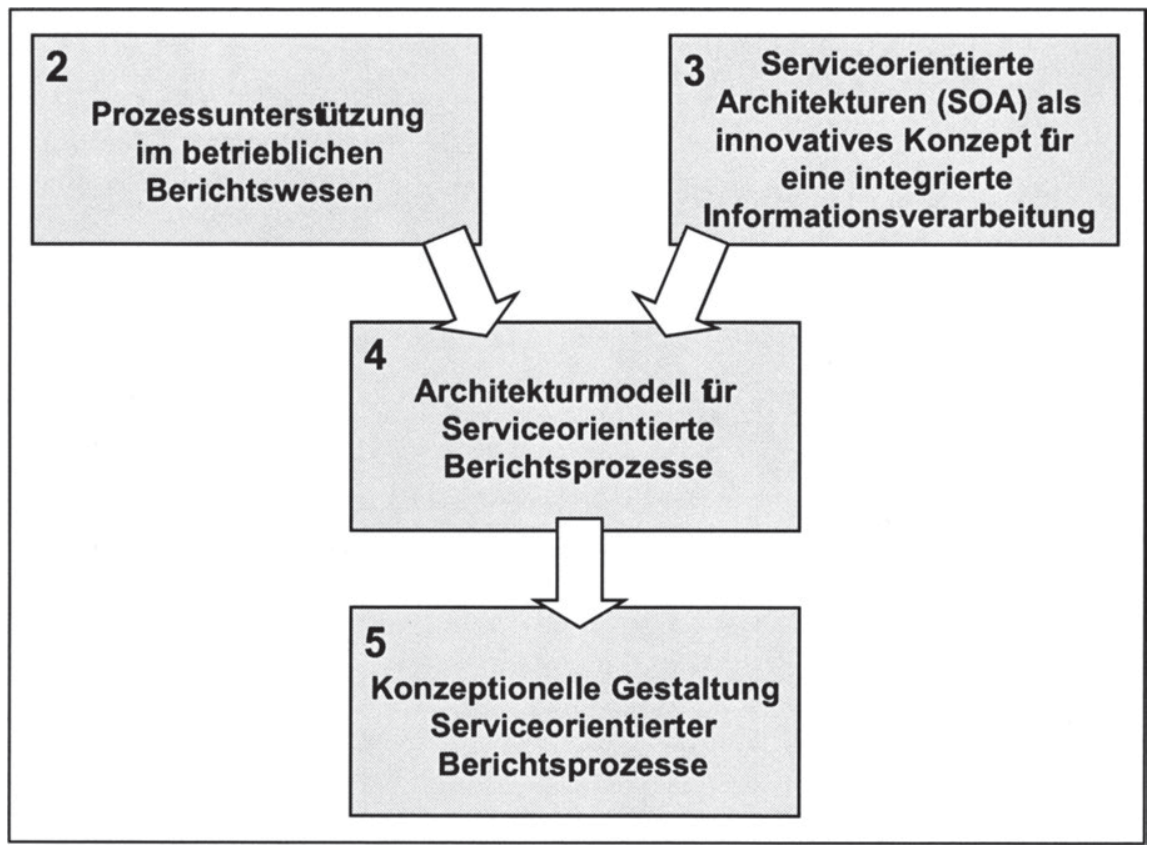

Abbildung 1: Grobgliederung der Untersuchung

Gegenstand des Kapitels 4 ist die Darstellung eines Architekturmodells, das sich für die Gestaltung Serviceorientierter Berichtsprozesse nutzen lässt. Zunächst wird hierzu der Begriff „Serviceorientierter Berichtsprozess (SOBP)“ eingeführt. Um eine SOBP auf Basis einer SOA zu entwickeln, ist es erforderlich, ein Architekturmodell aufzustellen, das der Entwicklung und Realisierung einer derartigen Anwendung zugrunde liegen soll. Die Herausforderung, ein geeignetes Architekturmodell zur Gestaltung eines SOBP zu entwerfen, besitzt eine große Bedeutung, da ein SOBP einen von vielen zu unterstützenden Prozessen darstellt, die sich auf Basis einer SOA automatisieren und/oder flexibilisieren lassen. Vor diesem Hintergrund sind Überlegungen zum architektonischen Aufbau einer SOA auch grundsätzlicher Art und unabhängig von der zu unterstützenden prozessorientierten Anwendung anzustellen. Als Ergebnis wird ein SOA-Ebenenmodell präsentiert, das zur Gestaltung und Ausführung Serviceorientierter Prozesse mehrheitlich in der Literatur favorisiert wird.

Die weiteren Ausführungen dieses Kapitels widmen sich der Frage, welche Erweiterungen am bestehenden SOA-Architekturmodell vorzunehmen sind, um die Informationsverarbeitungsprozesse im betrieblichen Berichtswesen durch die Ausführung von SOBP zu verbessern. Als Erweiterung des SOAEbenenmodells wird mit der Extensible Business Reporting Language (XBRL) 
ein Standard auf Basis der Extensible Markup Language (XML) vorgestellt und in das Architekturmodell integriert, der das Potenzial besitzt, unter semantischen Gesichtspunkten eine korrekte Verarbeitung der Geschäfts- und Finanzinformationen in einer SOA zu gewährleisten.

Nachdem in Kapitel 4 ein Architekturmodell für SOBP präsentiert wurde, stellt Kapitel 5 eine methodische Vorgehensweise vor, die zur konzeptionellen Gestaltung Serviceorientierter Berichtsprozesse zielführend ist. In diesem $\mathrm{Zu}$ sammenhang ist festzuhalten, dass sich generell für die methodische Entwicklung von Services, die in einer SOA genutzt werden sollen, bisher keine einheitliche Vorgehensweise durchsetzen konnte. ${ }^{4}$ Im ersten Teil dieses Kapitels werden zunächst bestehende Vorgehensstrategien, die im Zusammenhang mit der Einführung oder Entwicklung Serviceorientierter Anwendungen diskutiert werden, hinsichtlich ihrer Eignung untersucht, für die konzeptionelle Gestaltung von SOBP eingesetzt zu werden. Darauf aufbauend wird im zweiten Teil detailliert ein Ansatz präsentiert, der sich zur konzeptionellen Gestaltung von SOBP nutzen lässt. 
Alexander Pastwa - 978-3-631-75488-7

Downloaded from PubFactory at 01/11/2019 04:20:29AM

via free access 


\section{Prozessunterstützung im betrieblichen Berichtswesen}

Dass das betriebliche Berichtswesen ein wichtiges Instrument zur Unterstützung der Ereignisdokumentation, Planung, Steuerung und Kontrolle von Unternehmungen darstellt, 5 ist eine Einschätzung, die in der betriebswirtschaftlichen Literatur geteilt wird. ${ }^{6}$ Die Notwendigkeit, aussagekräftige und verlässliche $\mathrm{Be}-$ richte zu produzieren, gewinnt an Bedeutung, da die Shareholder und Stakeholder einer Unternehmung steigende Informationsanforderungen artikulieren. ${ }^{7} \mathrm{Als}$ Zugangsmedium und originäre Informationsquelle ist insbesondere das Internet in den Vordergrund gerückt. ${ }^{8}$ Mit Blick auf die wachsende Bedeutung internationaler Kapitalmärkte für die Eigenkapitalbeschaffung wird gefordert, das Berichtswesen stärker am Informationsbedarf der Investoren auszurichten. ${ }^{9}$

Aus der Perspektive der Wirtschaftsinformatik als interdisziplinäre Forschungsdisziplin zwischen der Betriebswirtschaftslehre und der Informatik, ${ }^{10}$ die sich mit der Gestaltung von Anwendungs- und Entscheidungsunterstützungssystemen befasst, ${ }^{11}$ interessiert die Frage, durch welche geeigneten fachlichen Konzepte und informationstechnischen Lösungen sich die Verarbeitung der von einer Unternehmung berichteten sowie zu berichtenden Informationen verbessern lässt. ${ }^{12}$ In einem allgemeinen Verständnis umfasst die Informations-

5 Die in der Literatur stattfindende Diskussion um die Begriffe Unternehmen und Unternehmung wird in dieser Arbeit nicht vertieft. Mit Blick auf die in der Literatur gewählte Terminologie werden beide Begriffe in den folgenden Ausführungen synonym verwendet. Zur Diskussion dieser Termini vgl. Kuhn (1990), S. 1

6 Vgl. dazu Becker/Köster/Sandmann (2006), S. 501; Blohm (1980), S. 317; Göpfert (2002), S. 147; Horváth (2006), S. 584. Da die Ausführungen dieser Arbeit sich ausschließlich auf das Berichtswesen von und innerhalb von Unternehmungen fokussieren, wird im Text zur Vermeidung einer sprachlichen Monotonie gelegentlich auf das Adjektiv „,betriebliches“ verzichtet.

7 Vgl. Gluchowski/Kemper (2006), S. 18. Zum Stakeholder- und Shareholderbegriff vgl. Abschnitt 2.1.2.

8 Vgl. Mertens (1999), S. 409; Lymer (1999), S. 289; Xiao/Jones/Lymer (2002), S. 245; Wagenhofer (2003), S. 262. Zu den Vorteilen und Problembereichen der Internetpublizität vgl. Schruff/Kayser (2002), S. $353 \mathrm{ff}$.

9 Vgl. Nutz/Strauß (2002), S. 447. RIDDER/BOMMER weisen in diesem Zusammenhang darauf hin, dass die Finanzierungsfähigkeit einer Unternehmung zu geringen Kapitalkosten maßgeblich von der Zufriedenheit der Kapitalmarktteilnehmer abhängt. Vgl. Ridder/Bommer (2006), S. 615.

10 Zum Gegenstandsbereich der Wirtschaftsinformatik vgl. Hansen/Neumann (2005), S. $101 \mathrm{ff}$.

11 Vgl. Buhl (2008), S. 433; Strangmeier (2008), S. 448.

12 Dass die Verbesserung des Berichtswesens eine Herausforderung für die Wirtschaftsinformatik darstellt, wird deutlich bei Becker/Seidel/Janiesch (2008), S. 229. 
verarbeitung alle Aktivitäten, die die zweckbezogene Informationsgewinnung und -verwendung sowie die Informationstransformation zum Gegenstand haben. ${ }^{13}$ Werden Informationen maschinell transformiert, so ist dieser Vorgang als Datenverarbeitung zu bezeichnen. ${ }^{14}$ Grundsätzlich lassen sich im betrieblichen Berichtswesen jegliche Arten von Informationen verarbeiten, die innerhalb einer Unternehmung anfallen. Die folgenden Ausführungen fokussieren dabei die Verarbeitung von Geschäfts- und Finanzinformationen.

Die effiziente und effektive Verarbeitung derartiger Informationen besitzt insofern eine Relevanz für eine berichtende oder berichtsempfangende Unternehmung, ${ }^{15}$ als einmal produzierte Inhalte - beispielsweise in Form eines Jahresabschlusses - zwischen verschiedenen Berichtsempfängern ausgetauscht und von diesen (weiter) verarbeitet werden können oder müssen. ${ }^{16}$ In Anlehnung an den Supply Chain-Gedanken wird die sich daraus ergebende Berichtslieferkette auch als Business Supply Chain, Business Reporting Supply Chain, Corporate Reporting Supply Chain, eReporting Supply Chain oder als Financial Supply Chain bezeichnet. ${ }^{17}$ Die bei der Verarbeitung der Informationen anfallenden Aufgaben umfassen die Interpretation, Aufbereitung, (Re-)Formatierung, Ein-

Mit Blick auf die Begriffsauslegung von GABRIEL/BEIER lässt sich diese Abgrenzung als Informationsverarbeitung i. w. S. auffassen. Vgl. Gabriel/Beier (2003), S. 34f.

Vgl. Gabriel/Beier (2003), S. 35. Daten und Informationen lassen sich mit Hilfe der Semiotik unterscheiden. Die Semiotik beschäftigt sich als allgemeine Sprach- und Zeichentheorie mit der syntaktischen, semantischen und pragmatischen Ebene von sprachlichen und nicht-sprachlichen Zeichensystemen. Während sich die Syntaktik mit den Regeln zur Verknüpfung von Zeichen befasst, beschäftigt sich die Semantik mit der inhaltlichen Bedeutung der Zeichen. Gegenstand der Semantik sind die Beziehungen zwischen den Zeichen und dem bezeichneten realen Objekt. Die Pragmatik fokussiert die Zweckorientierung von Informationen. Zu den Grundlagen der Semiotik vgl. Gabriel/ Beier (2003), S. 29ff. Daten bilden z. B. in Form einer Dezimalzahl, die aus den Ziffern „6“, „3“ und „9“ sowie dem Sonderzeichen „"“ entsprechend der syntaktischen Regeln zusammengesetzt ist, einzelne objektive Fakten zu Vorgängen oder Ereignissen ab. Indem eine Beziehung zur Realität hergestellt wird und die Daten in einen Kontext eingeordnet werden, entstehen aus Daten Informationen im Sinne der semantischen Ebene der Semiotik. Aus der Dezimalzahl 6,39 entsteht somit eine Kennzahl, wenn eine sachliche Beschreibung durch eine Dimensionsangabe wie z. B. Return on Investment (ROI) hinzugefügt wird.

15 Zum Effizienz- und Effektivitätsbegriff vgl. Abschnitt 2.3. Der Begriff der Gestaltung steht in diesem Zusammenhang für den gesamten Prozess der systematischen Entwicklung eines IS, wobei sich als Teilschritte dieses Prozesses die Phase der Planung, der Entwicklung, der Integration in eine Anwendungsumgebung sowie die Phase des Einsatzes identifizieren lassen. Vgl. Gluchowski/Gabriel/Chamoni (1997), S. 63.

16 Vgl. dazu Pandrangi (2003).

17 Vgl. Farewell (2006), S. 161; Ramin/Kesselmeyer/Ott (2006a), S. 179; Brown/Willis (2003), S. 70; Meyer-Pries/Gröner (2002), S. 53; Baars (2005), S. 194. 
gabe und Validierung der berichteten Informationen. ${ }^{18}$ Ferner kommt bei der Erstellung, Weitergabe und Aufnahme der Berichte eine Reihe von Personen, Informationssystemen (IS) und Daten- bzw. Dateiformate zum Einsatz, die eine schnelle und verlässliche Verarbeitung der Berichte erschweren. ${ }^{19}$ Als Berichte bzw. Reports kommen sämtliche Dokumente in Frage, in denen betriebswirtschaftliche Sachverhalte aufbereitet, bereinigt und miteinander verknüpft sind mit dem Ziel, die unternehmungsinternen und/oder -externen Adressaten mit relevanten und korrekten Informationen zu versorgen und sie damit bei der Erfüllung ihrer Aufgaben zu unterstützen. ${ }^{20}$ Die Berichte verarbeiten Informationen, die in den betrieblichen IS vorgehalten werden. ${ }^{21}$

In diesem Zusammenhang fordert GÖPFERT, beim betrieblichen Berichtswesen logistische Konzepte einzubeziehen mit dem Ziel, die effektive und effiziente Informationsversorgung der unternehmungsinternen und -externen $\mathrm{Be}$ richtsempfänger zu unterstützen. ${ }^{22}$ Losgelöst von der Frage, ob die Berichtsempfänger sich innerhalb oder außerhalb einer berichtenden Unternehmung befinden, hat die Forderung, logistische Konzepte für die Zwecke des betrieblichen Berichtswesens zu berücksichtigen, zur Konsequenz, den Fokus nicht nur auf die bloße informationstechnische Unterstützung der anfallenden Aufgaben bei der Verarbeitung der berichteten sowie zu berichtenden Informationen auszurichten. Gleichzeitig ist die bessere Koordination aller Verarbeitungsaktivitäten der berichtsanbietenden sowie -nachfragenden Unternehmungseinheiten zu

Vgl. Brown/Willis (2003), S. 70.

19 In der vorliegenden Arbeit orientiert sich der Begriff eines computergestützten IS an der Definition von HANSEN/NEUMANN. Dieser Begriffsauslegung zufolge handelt es sich bei einem computergestützten IS um ein System, „bei dem die Erfassung, Speicherung, Übertragung und/oder Transformation von Information durch den Einsatz der Informationstechnik teilweise automatisiert ist“. Hansen/Neumann (2005), S. 85. Zur Vermeidung einer sprachlichen Monotonie wird in dieser Arbeit der Begriff IT-Systeme synonym verwendet. Als soziotechnisches System setzt sich ein IS aus menschlichen und maschinellen Aufgabenträgern zusammen. Da die Kommunikation zwischen den Systemelementen eine systemimmanente Eigenschaft eines IS darstellt, wird im weiteren Verlauf dieser Arbeit die alternative Bezeichnung Informations- und Kommunikationssystem (IuK-System) nicht verwendet. Vgl. Fink/Schneidereit/Voß (2005), S. 3. Abzugrenzen sind rechnergestützte IS von den Anwendungssystemen, die im vorliegenden Arbeitbericht auch als Anwendungen, Applikationen oder betriebliche IS bezeichnet werden. Diese Systeme haben die computergestützte Unterstützung eines betrieblichen Anwendungs- bzw. Aufgabenbereichs zum Ziel und lassen sich daher neben den Hardwaresystemen als Teile eines IS auffassen. Vgl. Fink/Schneidereit/Voß (2005), S. 3f. Siehe dazu die Berichtszwecke in Abschnitt 2.1.3. Damit folgt das dieser Arbeit zugrunde liegende Verständnis eines Berichts der Vorstellung von Kemper/Mehanna/ Unger (2004), S. 110.

Vgl. Göpfert (2002), S. 155. 
fordern. Damit rückt die Gestaltung der Berichtsprozesse in den Vordergrund der Betrachtung.

Das vorliegende Kapitel widmet sich der Prozessunterstützung im betrieblichen Berichtswesen. ${ }^{23}$ Der Fokus liegt dabei sowohl auf der Erarbeitung begrifflicher, konzeptioneller und technischer Grundlagen einer Prozessunterstützung im Berichtswesen als auch auf der Diskussion aktueller Problemfelder, die eine effiziente und effektive Ausführung der Berichtsprozesse verhindern.

Abschnitt 2.1 widmet sich zunächst den wichtigsten Terminologien und herkömmlichen Sichtweisen auf das betriebliche Berichtswesen. Aus der Perspektive der Wirtschaftsinformatik interessiert die Frage, wie Berichtssysteme aufgebaut und ausgeprägt sein müssen, um die Ausführung der Berichtsprozesse zu ermöglichen. In diesem Zusammenhang ist zu klären, welche Kernprozesse sich im betrieblichen Berichtswesen identifizieren lassen. Diese Aspekte werden in Abschnitt 2.2 thematisiert. Der darauf folgende Abschnitt 2.3 fokussiert aktuell zu beobachtende Defizite, die die Ausführung von Berichtsprozessen behindern. Die Störungsursachen betreffen sowohl die Effektivität als auch die Effizienz der Informationsverarbeitung innerhalb der Berichtsprozesse. Das Kapitel schließt in Abschnitt 2.4 mit einer Zusammenfassung und Würdigung der Prozessorientierung im Berichtswesen.

\subsection{Terminologische und konzeptionelle Grundlagen zum IT-gestützten Berichtswesen}

Das Berichtswesen steht schon seit vielen Jahren im Fokus einer wissenschaftlichen Auseinandersetzung. Abschnitt 2.1.1 obliegt daher zunächst die Vorstellung und Erörterung verschiedener Sichtweisen zum Begriff des Berichtswesens. Abschnitt 2.1.2 verfolgt die Zielsetzung, das betriebliche Berichtswesen von konkurrierenden Termini abzugrenzen, zu denen die Unternehmenspublizität sowie die Rechnungslegung gehören. In Abschnitt 2.1.3 schließt sich die Darstellung der Zwecke an, die sich mit dem betrieblichen Berichtswesen verfolgen lassen.

23 In einem allgemeinen Verständnis lässt sich ein Prozess als eine Folge von logischen, miteinander verbundenen Einzelfunktionen auffassen. Vgl. Krcmar (2003), S. 99. Aus betriebswirtschaftlicher Perspektive ist die Gestaltung von Geschäftsprozessen (business processes) von Bedeutung. Als Synopse ausgewählter Definitionsvorschläge lassen sich Geschäftsprozesse als betriebswirtschaftlich relevante Prozesse verstehen, die über eine zusammengehörige Abfolge von zeitlich und sachlogisch gegliederten Funktionen und Ereignissen der Erstellung einer Leistung dienen, die von einem internen oder externen Kunden angefordert und abgenommen wird. Vgl. Scheer/Thomas (2005), S. 1069; Balzert (2001), S. 126; Fink/Schneidereit/Voß (2005), S. 95; Hansen/Neumann (2005), S. 233. 


\subsubsection{Auslegungen des Berichtswesens}

Unter den Vorschlägen zur Abgrenzung des Berichtswesens, dessen englischsprachiges Pendant als Reporting bezeichnet wird,24 steht einem engen Definitionsansatz eine weite Begriffsauslegung gegenüber. Nach dem Verständnis von BLOHM als Vertreter einer weiten Sichtweise steht das Berichtswesen für die Gesamtheit aller Einrichtungen, Mittel und Maßnahmen, die darauf ausgerichtet sind, Informationen über den Betrieb und seine Umwelt zu erarbeiten, weiterzuleiten oder zu verarbeiten. ${ }^{25}$ Ähnlich kommt die weite Sichtweise des Berichtswesens bei KüPPER zum Ausdruck. Seiner Auffassung nach sind unter Berichtswesen alle Personen, Organisationseinheiten, Vorschriften, Daten und Prozesse zu verstehen, die bei der Produktion sowie der Weitergabe der Berichte maßgeblich sind. ${ }^{26}$ In beiden Perspektiven wird deutlich, dass für die Gestaltung des Berichtswesens eine ganzheitliche Herangehensweise erforderlich ist, im Rahmen dieser neben den zu verarbeitenden Berichtsinformationen auch die Organisationsstruktur sowie die anfallenden Prozesse zur Verarbeitung der Berichte zu berücksichtigen sind. Damit umfasst diese Sichtweise sowohl den Aufbau als auch den Ablauf des Berichtswesens.

Im Vergleich zum weiten Begriffsansatz nach BLOHM und KüPPER lässt sich das Berichtswesen nach HORVÁTH enger fassen. ${ }^{27}$ Seiner Vorstellung zufolge steht das Berichtswesen für die Phase der Informationsübermittlung i. w. S. Als Informationsübermittlung i. w. S. versteht HORVÁTH die Erstellung und Weiterleitung von Berichtsinformationen. Darüber hinaus beschränkt sich die enge Sichtweise von HORVÁTH auf die Unternehmungsangehörigen als alleiniger Adressatenkreis der Berichte, wobei unter dieser Gruppe die Manager als Zielgruppe herausragen. Mit Blick auf die Art der berichteten Informationen sind für den Austauschprozess insbesondere diejenigen Informationen von Interesse, die der Planung und Kontrolle als wichtige Aufgaben des Managements dienen. ${ }^{28}$

BECKER/SEIDEL/JANIESCH bemängeln, dass sowohl die weite als auch die enge Auslegung des Berichtswesens nicht berücksichtigen, dass eine bedarfsge-

In Anlehnung an BECKER/SEIDEL/JANIESCH und GLUCHOWSKI/GABRIEL/DITTMAR werden die Begriffe Berichtswesen und Reporting im Folgenden synonym verwendet. Vgl. dazuBecker/Seidel/Janiesch (2007), S. 605; Gluchowski/Gabriel/Dittmar (2007), S. 205. Vgl. Blohm (1982), S. 866.

Vgl. Küpper (1997), S. 148.

Vgl. Horváth (2006), S. 583.

28 Der vorliegenden Arbeit liegt ein funktional geprägtes Managementverständnis zugrunde. Im Vergleich zur institutionellen sowie aktivitätsorientierten Sicht stellt die funktionelle Perspektive alle Managementhandlungen in den Vordergrund, die sich auf Entscheidungen beziehen. Entscheidungen zu treffen, diese durchzusetzen und zu hinterfragen sowie die Verantwortung für die Entscheidungen zu übernehmen, sind demnach typische Aufgaben eines Managers. Vgl. Scherm/Pietsch (2008). S. 430f. 
rechte Informationsversorgung nur gewährleistet werden kann, wenn die herkömmlichen Berichte um geeignete Analyse- und Auswertungssichten ergänzt werden. ${ }^{29}$ Folglich erweitern die Autoren das von BLOHM und HORVÁTH geprägte Verständnis zum Berichtswesen um die Notwendigkeit, geeignete Analyse- und Auswertungsperspektiven auf einen verdichteten Datenbestand zur Verfügung zu stellen, in dem alle relevanten Informationen abgelegt sind. ${ }^{30}$ Damit ist dieses Begriffsverständnis im Vergleich zu den vorangehenden Sichtweisen durch eine stärkere Fokussierung auf die softwaretechnische Umsetzung des Berichtswesens geprägt. ${ }^{31}$

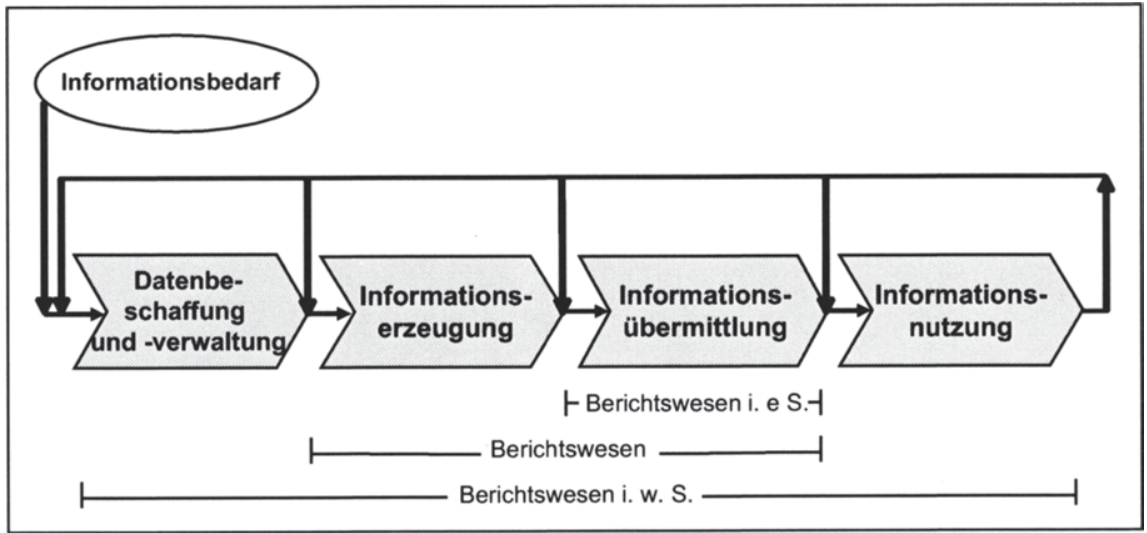

Abbildung 2: Einordnung des Berichtswesens nach Göpfert ${ }^{32}$

Während im weiten Begriffsverständnis zum Berichtswesen sowohl eine strukturelle als auch eine ablauforientierte Komponente vereint sind, liegt beim Begriff der Berichterstattung der Fokus auf der ablauforientierten Komponente des Berichtswesens. In diesem Zusammenhang versteht BLOHM unter betrieblicher Berichterstattung die Vermittlung von Berichten, die über Tatsachen, Ereignisse, Zusammenhänge und Vorgänge aus dem Betrieb und seiner Umwelt informieren. ${ }^{33}$ Mit Blick auf dieses Begriffsverständnis ist zu konstatieren, dass die von BLOHM vorgeschlagene Abgrenzung zur Berichterstattung mit der engen

29 Vgl. Becker/Seidel/Janiesch (2007), S. 607.

30 Als verdichtete Daten werden in dieser Arbeit alle Informationen verstanden, die je nach Informationsbedarf des Berichtsempfängers in konzentrierter bzw. angereicherter Form vorliegen.

31 Siehe dazu die unterschiedlichen Ausprägungen von Berichtssystemen in Abschnitt 2.2.1.2.

32 Vgl. Göpfert (2002), S. 146.

33 Vgl. Blohm (1980), S. 316. 
Sichtweise des Berichtswesens korrespondiert, wobei der Autor im Gegensatz zu HORVÁTH nicht explizit auf die Manager als alleinige Zielgruppe der Informationsversorgung Bezug nimmt.

Im Vergleich zu den alternativen Sichtweisen präsentiert GÖPFERT ein prozessorientiertes Begriffsverständnis. Wie in der Abbildung 2 zu erkennen ist, zeichnet sich der Autorin zufolge das weite Begriffsverständnis im Vergleich zur engen Begriffsauslegung durch einen stärkeren Bezug zum Informationsprozess aus. Im Gegensatz zum engen Begriffsverständnis nach HORVÁTH umfasst das Berichtswesen im weitesten Sinne nicht nur die Phase der Informationsübermittlung, sondern die Phasen Datenbeschaffung und -verwaltung, Informationserzeugung und Informationsnutzung. Zwischen diesen beiden Auslegungen lässt sich nach GÖPFERT der Terminus des Berichtswesens einordnen, dessen Kernphasen, wie von HORVÁTH propagiert, die Berichtserzeugung und Berichtsdistribution sind. ${ }^{34}$

\subsubsection{Abgrenzung des Berichtswesens}

Unabhängig von den in Abschnitt 2.1.1 thematisierten Auslegungen des Berichtswesens lässt sich das Reporting eindeutig von anderen betriebswirtschaftlichen Instrumenten abgrenzen, die die Informationsverarbeitung von betrieblichen Informationen fokussieren. Gegenstand der Betrachtung sind in den folgenden Ausführungen die Instrumente der Rechnungslegung und Unternehmenspublizität.

Wie in der Abbildung 3 deutlich wird, lässt sich die Unternehmenspublizität anhand der Informationsinhalte vom Terminus der Rechnungslegung abgrenzen. ${ }^{35}$ In diesem Zusammenhang bezeichnet die Unternehmenspublizität die Bekanntmachung von Unternehmungsinformationen, die von einer unbestimmten Anzahl von Adressaten eingesehen werden können. ${ }^{36}$ Im Fokus steht dabei die Vermittlung von öffentlichen Informationen, die aus einer gesetzlichen Verpflichtung heraus oder auf freiwilliger Basis der Öffentlichkeit zugänglich gemacht werden. ${ }^{37}$

Vgl. Göpfert (2002), S. 144.

35 Vgl. Vielmeyer (2004), S. 14.

36 Vgl. Pellens/Fülbier/Gassen (2006), S. 841. An dieser Stelle ist darauf hinzuweisen, dass nicht die tatsächliche Kenntnisnahme bzw. Inanspruchnahme der Unternehmensinformation entscheidend ist, sondern allein die Möglichkeit, die beispielsweise auf der Website der Unternehmung veröffentlichten Informationen einzusehen, für das Begriffsverständnis der Unternehmenspublizität von Bedeutung ist.

37 Dem Systematisierungsvorschlag von PELLENS/FüLBIER/GASSEN zufolge lässt sich die Unternehmenspublizität anhand der Kriterien „Grund der Veröffentlichung“, „Zeitpunkt und -raum der Veröffentlichung“ sowie „Inhalt der Veröffentlichung“ einteilen. Vgl. Pellens/Fülbier/Gassen (2006), S. 846f. 


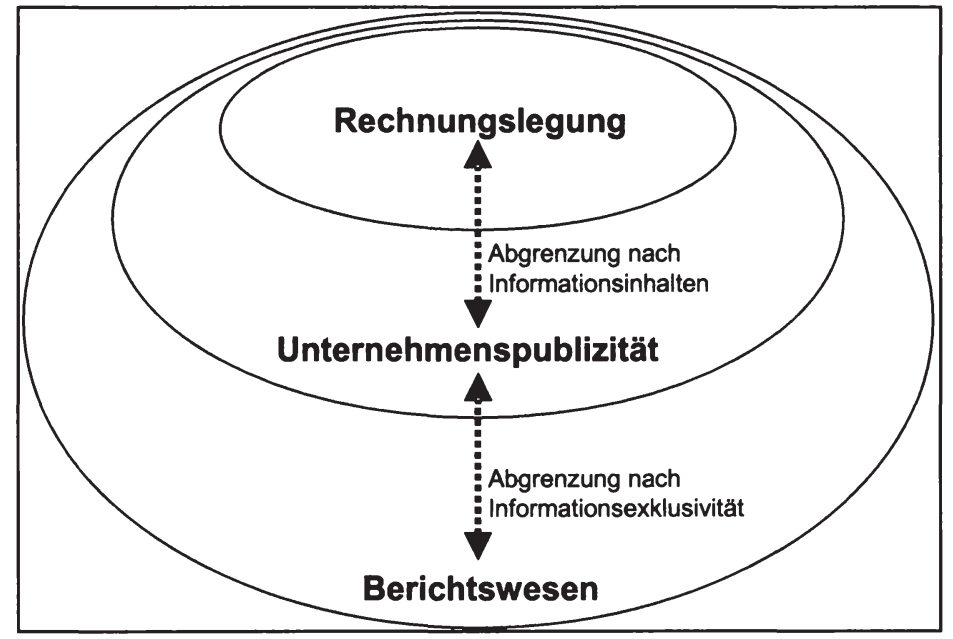

\section{Abbildung 3: Abgrenzung der Begriffe Berichtswesen, Unternehmenspublizität und Rechnungslegung 38}

Die Rechnungslegung, die auch als externes Rechnungswesen oder Financial Accounting bezeichnet wird, verfolgt hingegen das Ziel, unter Anwendung diverser Vorschriften die Vermögens-, Finanz- und Ertragslage der Unternehmung abzubilden und auf diese Weise den Außenstehenden Rechenschaft über die wirtschaftliche Entwicklung abzulegen. Daraus folgt, dass in der Rechnungslegung nur quantifizierbare Informationen verarbeitet werden, die objektivierbar sind und in allgemeine Instrumente, zu denen z. B. die Bilanz oder die Gewinn- und Verlustrechnung (GuV-Rechnung) gehören, münden. ${ }^{39}$ Unternehmungsinformationen, die Gegenstand der Unternehmenspublizität sind, gehen über die Rechnungslegungsdaten hinaus und umfassen z. B. die Selbstdarstellungen der Unternehmung oder die Einladung zur Hauptversammlung in einem Börsenpflichtblatt.40

Mit Blick auf die Abbildung 3 kann die Ausrichtung nach dem Empfängerkreis der zu veröffentlichenden Unternehmungsinformationen dazu genutzt werden, das betriebliche Berichtswesen von der Unternehmenspublizität abzu-

38 In Anlehnung an Vielmeyer (2004), S. 14.

39 Vgl. Vielmeyer (2004), S. 14.

40 Vgl. Pellens/Fülbier/Gassen (2006), S. 841. 
grenzen. Das separierende Kriterium richtet sich auf die Exklusivität der Informationsbereitstellung. In Abgrenzung zum Begriff der Unternehmenspublizität zeichnet sich das Berichtswesen einer Unternehmung durch ein umfassendes Verständnis aus. Gegenstand des Berichtswesens ist es, sowohl öffentliche als auch private Informationen den jeweiligen Anspruchsgruppen einer Unternehmung in Form von geeigneten Berichten zur Verfügung zu stellen. Im Vergleich zu den öffentlichen Informationen zeichnen sich private Informationen durch einen höheren Exklusivitätsgrad bei der Informationsgewährung aus, da sie nur von einem bestimmten Adressatenkreis verarbeitet werden können. ${ }^{41}$ Generell kommen als potenzielle Berichtsempfänger beispielsweise das Management, die Shareholder sowie die Stakeholder einer Unternehmung in Frage. ${ }^{42}$ Als Stakeholder werden alle unternehmungsinternen und -externern Anspruchs- bzw. Interessensgruppen einer Unternehmung bezeichnet. ${ }^{43}$ Die Einteilung des $\mathrm{Be}-$ richtswesens nach dem Empfängerkreis der Berichte ist in diesem Zusammenhang das bekannteste Systematisierungskriterium des Reportings. ${ }^{44}$

Richtet sich das Reporting auf die Informationsversorgung unternehmungsinterner Angehöriger, wird von internem Berichtswesen gesprochen. Im Mittelpunkt der Informationsversorgung stehen die Manager der Unternehmung. Das externe Reporting hingegen adressiert den Informationsbedarf von Akteuren, die sich außerhalb einer Unternehmung befinden. ${ }^{45}$ Als unternehmungsexterne Stakeholder lassen sich z. B. Kunden, Lieferanten, konkurrierende Unternehmungen, Medien, der Staat (insbesondere in Form der Kartellbehörde), die öffentliche Meinung sowie Arbeitgeber- und Arbeitnehmerorganisationen identifizieren. ${ }^{46}$ Darüber hinaus richtet sich das externe Berichtswesen auch an die Anteilseigner bzw. die Shareholder einer Unternehmung.

Neben diesen Anspruchsgruppen kommen als potenzielle Berichtsempfänger auch Wirtschaftsprüfer in Frage. Bevor die Informationen des externen Rechnungswesens beispielsweise in Form eines Geschäftsberichts der Öffentlichkeit zur Verfügung gestellt werden, sind diese durch unabhängige Wirtschaftsprü-

41 Vgl. Gassen (2000), S. 10f.

42 Vgl. Gleich et al. (2002), S. 338.

43 Vgl. Schmidt/Agbor (2008), S. 357.

44 Vgl. dazu Blohm (1974), S. 16. Als weitere Kriterien kommen z. B. die Informationsart (Istwerte, Vorgabewerte, Prognosewerte, etc.), die Erscheinungsweise (regelmäßig, unregelmäßig, überjährig, unterjährig, etc.), die Informationsträger (CD, USB-Stick, Diskette, Bildschirm, Schriftstück, etc.) oder der Verdichtungsgrad (Ursprungswerte, Kennzahlen, etc.) in Frage. Vgl. Göpfert (2002), S. 148.

45 Der Definition von vOM BROCKE/BUDDENDICK folgend, können Akteure als handlungsfähige Einheiten verstanden werden, die vor dem Hintergrund ihres eigenen Zielsystems handlungsorientiert agieren und dabei ihre kommunikativen und kognitiven Fähigkeiten einsetzen. Vgl. vom Brocke/Buddendick (2004), S. 342. 
fer(-Gesellschaften) auf ihre Richtigkeit zu untersuchen. ${ }^{47}$ Neben den Wirtschaftsprüfern existiert mit den Informationsintermediären ein weiterer $\mathrm{Be}$ richtsempfängerkreis, der als Zielgruppe des Unternehmensreportings von Bedeutung sein kann. Die Aufgabe der Informationsintermediäre liegt darin, die von einer Unternehmung berichteten Informationen zu sammeln, aufzubereiten und in einem nächsten Schritt einer undifferenzierten oder ausgewählten Endverbrauchergruppe zur Verfügung zu stellen. Eine derartige Dienstleistung kann gegen Entgelt oder entgeltlos erfolgen. Als Informationsintermediäre kommen z. B. diverse Wirtschaftsinstitute, Wirtschaftsverbände, Gewerkschaften, Börsenplattformen, Nachrichtendienste oder Beratungsunternehmungen in Frage. Vor dem Hintergrund dieses Begriffsverständnisses schließt das betriebliche Berichtswesen auch die Unternehmenspublizität ein.

\subsubsection{Berichtszwecke}

Wie aus den bisherigen Ausführungen hervorgeht, zielt das Reporting auf die Informationsversorgung mit Hilfe von Berichten, in denen die entsprechenden Geschäfts- und Finanzinformationen präsentiert sind. ${ }^{48}$ In der Literatur werden hierzu drei Berichtszwecke genannt, die Gegenstand der folgenden Ausführungen sind. 49

1. Der erste Aufgabenbereich liegt in der Dokumentation von Ereignissen mit dem Ziel, das Betriebsgeschehen in einer strukturierten Form abzubilden. Der Berichtszweck der Dokumentation richtet sich auf alle Informationen, die aus einer rechtlichen bzw. gesetzlichen Verpflichtung heraus von einer Unternehmung protokolliert werden müssen. Eine entsprechende Verpflichtung zur Dokumentation von Berichten resultiert aus den entsprechenden Vorschriften des Handels-, Aktien-, Steuer- und Umweltrechts.

Neben dem Handelsgesetzbuch als Bilanzierungsstandard im deutschen Raum haben in den vergangenen Jahren insbesondere die International Accounting Standard / International Financial Reporting Standards (IAS/IFRS) als internationaler Rechnungslegungsstandard an Bedeutung gewonnen.50 Wird eine Aufnahme an der amerikanischen Börse angestrebt, ist nach den United States Generally Accepted Accounting Principles (US-GAAP) zu bilanzieren. ${ }^{51}$ Als einer der wichtigsten Berichte ist in diesem Zusammenhang der Jahresabschluss

Vgl. Wagenhofer (2003), S. 264.

48 Vgl. dazu auch Leßweng (2003), S. 335.

49 Vgl. Göpfert (2002), S. 147. HiRSCH/PAEFGEN/SCHAIER bemerken in diesem Zusammenhang, dass bei allen Berichtszwecken der übergeordnete Zweck der Informationsversorgung überwiegt. Vgl. Hirsch/Paefgen/Schaier (2008), S. 327. Vgl. Gluchowski/Gabriel/Dittmar (2007), S. 234.

51 Zu den Pflichten einer berichterstattenden Unternehmung aus der Perspektive von IFRS, US-GAAP und HGB vgl. Pellens/Fülbier/Gassen (2006), S. 127ff. 
zu nennen, der sich im Falle einer Anwendung von IFRS aus einer Bilanz, einer GuV-Rechnung, einer Kapitalflussrechnung, einer Eigenkapitalveränderungsrechnung sowie Anhangangaben zusammensetzt.52 Neben den Berichten, die aus einer gesetzlichen Dokumentationsverpflichtung heraus $\mathrm{zu}$ erstellen sind, leisten auch alle Dokumentationen, die eine Unternehmung freiwillig bzw. zur Selbstinformation generiert, einen wichtigen Beitrag zur Unternehmungssteuerung. 53

2. Ein weiterer Berichtszweck lässt sich in der Auslösung von Aktivitäten identifizieren. Dadurch, dass beispielsweise eine negative Abweichung gegenüber einem Plan-/Sollwert in einem entsprechenden Bericht angezeigt wird, sind die Voraussetzungen dafür geschaffen, dass eine Unternehmensführung frühzeitig Einfluss auf das Unternehmungsgeschehen nehmen kann. Wie unternehmungsinterne Entwicklungen zur Entscheidungsunterstützung sowie zur Ableitung von gegensteuernden Maßnahmen führen, so können auch unternehmungsexterne Berichte, die von oder über Konkurrenzunternehmungen erstellt werden, zu einer entsprechenden Reaktion der Unternehmungsleitung beitragen.

3. Der letzte Aufgabenbereich des Berichtswesens richtet sich auf die Vorbereitung und Kontrolle von Entscheidungen. ${ }^{54}$ Das Ziel des Reportings liegt darin, durch die Bereitstellung geeigneter Informationen alle Phasen des Managementprozesses zu begleiten und zu unterstützen. ${ }^{55}$ Einerseits lassen sich Berichte zur Entscheidungsfindung bzw. Entscheidungsvorbereitung einsetzen. ${ }^{56} \mathrm{Zum}$ anderen dienen die berichteten Informationen auch zur Unterstützung bei der Entscheidungsdurchsetzung bis hin zur Entscheidungskontrolle. ${ }^{57}$

Kommen für die Produktion und/oder Präsentation der Berichte verschiedene Softwaresysteme und Werkzeuge zum Einsatz, wird die daraus resultierende technische Gesamtlösung als (computergestütztes) Berichtssystem bezeichnet. ${ }^{58}$ Gegenstand des folgenden Abschnitts ist die Frage, welche grundlegende Struktur und Ausprägungen eines Berichtssystems sich zur Unterstützung des Reportings identifizieren lassen.

Vgl. Pellens/Fülbier/Gassen (2006), S. 151.

Vgl. Göpfert (2002), S. 147.

Vgl. Göpfert (2002), S. 147.

Als Phasen lassen sich in diesem Zusammenhang die Situationsanalyse, Planung i. e. S., Organisation und Steuerung sowie Kontrolle identifizieren. Vgl. Gluchowski/Gabriel/ Dittmar (2007), S. $21 \mathrm{ff}$.

Vgl. Gluchowski (2006), S. 210.

Vgl. Gluchowski (2006), S. 210; Göpfert (2002), S. 147.

Vgl. Gluchowski (2006), S. 208. BLOHM weist darauf hin, dass in der Literatur der Begriff Berichtssystem synonym zum Terminus Berichtswesen verwendet wird. Vgl. Blohm (1980), S. 315. Dieser Sichtweise wird in der vorliegenden Arbeit nicht gefolgt. Stattdessen werden Berichtssysteme als IT-gestützte IS zur Unterstützung der Berichtszwecke verstanden. 


\subsection{Berichtssysteme zur informationstechnischen Unterstützung von Be- richtsprozessen}

In der Auslegung des Berichtswesens nach GÖPFERT ist bereits deutlich geworden, dass das Berichtswesen im weitesten Sinne sich als Informationsprozess verstehen lässt. ${ }^{59}$ Ausgehend von der Vorstellung der Informationstechnik (Abschnitt 2.2.1), die heute zur Unterstützung der anfallenden Berichtsprozesse zum Einsatz kommt, werden in Abschnitt 2.2.2 die Kernprozesse des Berichtswesens präsentiert.

\subsubsection{Berichtssysteme als Anwendungsbereich von Business Intelligence}

Der folgende Abschnitt befasst sich mit der informationstechnischen Unterstützung des Berichtswesens. Dabei geht es im Abschnitt 2.2.1.1 zunächst um das Business Intelligence-Konzept, das sich als technischer und konzeptioneller Bezugsrahmen zur Gestaltung von Berichtsanwendungen etabliert hat. ${ }^{60}$ Die Frage nach einer geeigneten Klassifizierung von Berichtssystemen wird in Abschnitt 2.2.1.2 aufgegriffen.

\subsubsection{Business Intelligence-Systeme (BI-Systeme) als übergeordnete Systemkategorie zur Gestaltung und Einordnung von Berichts- anwendungen}

Obwohl die Begrifflichkeit Business Intelligence (BI) in den letzten Jahren das Stadium eines neuen IT-Schlagworts verlassen hat und eine Reihe BI-tauglicher Anwendungen implementiert wurden, ${ }^{61}$ ist zu konstatieren, dass in der Literatur nach wie vor eine Reihe von Definitionen und Begriffsauslegungen zu BI existieren. ${ }^{62}$ Bisher ist es nicht gelungen, aus diesen Vorschlägen eine allgemein akzeptierte Definition von BI zu etablieren. ${ }^{63}$ In einem integrierten Begriffverständnis lässt sich $\mathrm{BI}$ als Gesamtheit aller Methoden, Technologien und Prozes-

59 Vgl. Abschnitt 2.1.1.

60 NAVRADE und MUKSCH/BEHME zufolge stehen das Berichtswesen sowie die betrieblichen Berichtssysteme für den informationsorientierten Anwendungsbereich von Business Intelligence-Systemen. Vgl. Navrade (2006), S. 12; Muksch/Behme (2001), S. 23f.

61 Vgl. dazu Gluchowski (2001), S. 5. Erfolgreiche BI-Projekte präsentieren z. B. Gabriel/Hoppe/Pastwa (2008), S. 287ff.; Hoppe/Pastwa (2007), S. 39ff.; Grothe (2005), S. $138 \mathrm{ff}$.

62 Vgl. Leßweng (2004), S. 42; Liebowitz (2006), S. 19. Siehe dazu die Übersicht über verschiedene BI-Definitionen bei Kemper/Mehanna/Unger (2004), S. 2ff. Einen Ordnungsrahmen zur Positionierung eines engen, analyseorientierten und weiten BIVerständnisses präsentiert Gluchowski (2001), S. 7 f. 
se mit entscheidungsunterstützendem Charakter verstehen, die zur besseren Einsicht in das eigene Geschäft und damit zum besseren Verständnis in die Mechanismen relevanter Wirkungsketten führen. ${ }^{64}$ Mit Blick auf diese Abgrenzung des BI-Begriffs steht dieser für eine begriffliche Klammer, die eine Vielzahl unterschiedlicher Ansätze, Werkzeuge sowie Anwendungen zur Analyse geschäftsrelevanter Daten zu bündeln versucht. ${ }^{65} \mathrm{Zu}$ diesen gehört beispielsweise der Einsatz eines Data Warehouse (DWH), das die zentrale und einheitliche Datenbasis für die Implementierung eines BI-Systems repräsentiert. ${ }^{66}$ Dabei fokussiert BI die integrierte Nutzung sowohl strukturierter als auch unstrukturierter Informationen, um eine möglichst fundierte Entscheidungsunterstützung zu gewährleisten. 67

I. d. R. liegen die Daten einer Unternehmung in verschiedenen Systemen vor, so dass eine Zusammenführung der Daten erforderlich ist.68 Wie sich der Abbildung 4 entnehmen lässt, sind auf der untersten Ebene der BI-Referenzarchitektur diverse unternehmungsinterne operative Vorsysteme sowie potenzielle externe Datenquellen positioniert. Sie dienen als Datenlieferanten für das DWH, das als Datenspeicher Bestandteil der mittleren Schicht ist.

Ein DWH stellt eine einheitliche, vollständige und konsistente Sammlung von Daten dar, die aus verschiedenen Quellen stammen und von verschiedenen Anwendern genutzt werden können. ${ }^{69}$ Die Daten aus den Vorsystemen werden in bestimmten zeitlichen Abständen im DWH zusammengeführt. ${ }^{70}$ Das DWH wird neben den operativen Systemen bzw. Online-Transaction-ProcessingSystemen (OLTP-Systemen) betrieben. Letztere sind auf die Verarbeitung von Massendaten sowie die Ausführung zeitkritischer, in großer Anzahl vorkommender Transaktionen ausgerichtet. ${ }^{71}$ Im Gegensatz zu diesen Systemen

Vgl. Gluchowski (2001), S. 14.; Dittmar/Schulze (2006), S. 27; Chamoni/Linden (2007), S. 1588; Wenzke (2005), S. 631; Wall (2008), S. 82. Das umfassende BIVerständnis integriert sowohl den klassischen als auch den prozessorientierten BIBegriff. Während der klassische BI-Begriff tendenziell für ein statisches, vergangenheitsbezogenes Reporting und die Analyse von Fehlentwicklungen steht, fokussiert die prozessorientierte Perspektive die Planung, Verbesserung und Steuerung von Prozessen. Vgl. Seufert/Schiefer (2008), S. 21.

Vgl. Gluchowski/Gabriel/Dittmar (2007), S. 93.

66 Vgl. Kruppke/Bauer (2005), S. 79.

67 Vgl. Raimann/Mähler (2006), S. 39.

68 Vgl. Hahn (2003), S. 605.

69 Vgl. Devlin (1997), S. 20.; Frosch-Wilke (2003), S. 597.

70 Vgl. Stock/Düsing (2003), S. 186.

71 Vgl. Härder/Rahm (2001), S. 500. 
stellt ein DWH die Datenbasis für dispositive und strategische Aufgaben zur Verfügung. ${ }^{72}$

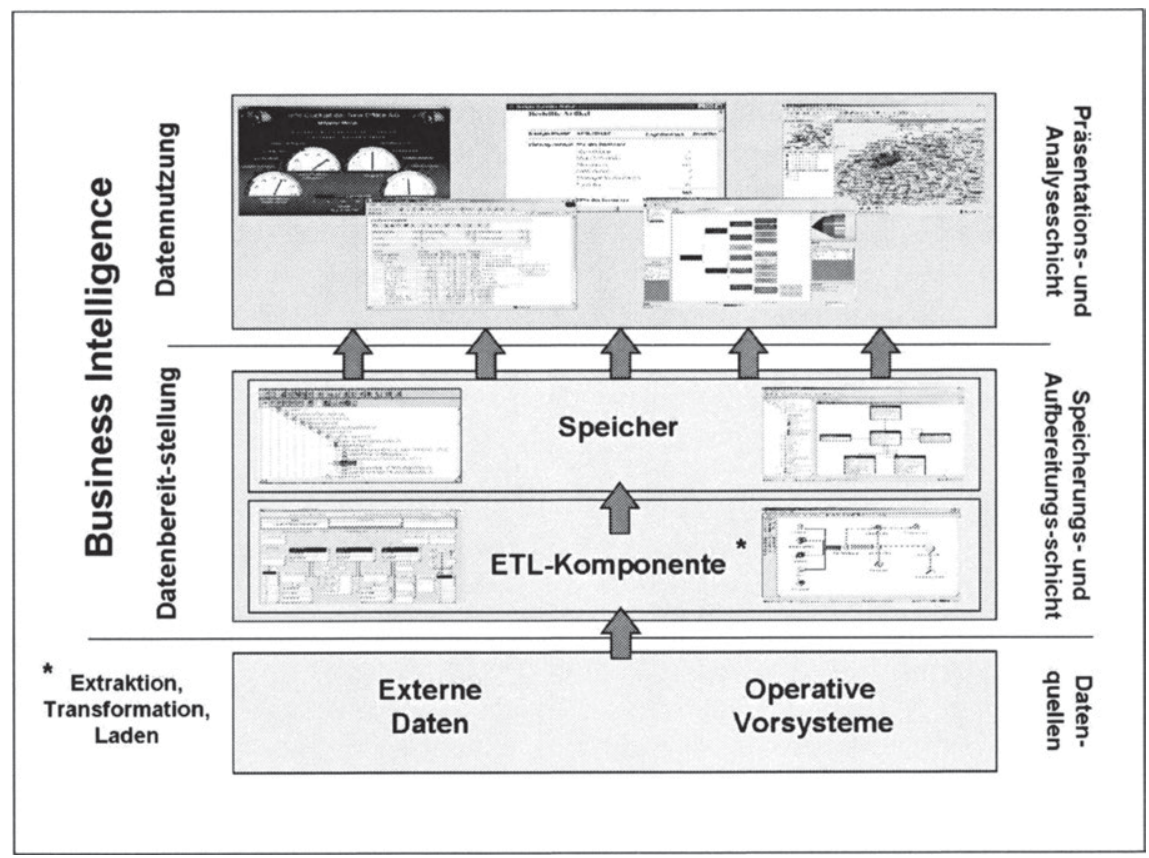

Abbildung 4: Aufbau eines BI-Systems ${ }^{73}$

Ein Transfer der Daten aus den Vorsystemen in das DWH erfolgt unter dem Einsatz einer ETL-Komponente, die für die Teilschritte Extract (Extraktion), Transform (Transformation) und Load (Laden) steht. ${ }^{74}$ Als Schnittstelle zwischen den Vorsystemen und dem DWH bildet diese einen weiteren Baustein der BI-Architektur. Dabei ist die ETL-Komponente für die Umwandlung der heterogenen Daten in einen konsistenten und homogenen Bestand sowie das Laden dieser Daten in das DWH verantwortlich. Der Teilschritt der Extraktion sorgt dabei für die Selektion und Bereitstellung der Quelldaten für den weiteren Pro-

72 Vgl. Petersohn (2005), S. 40. Zu den Auswirkungen und Nutzungspotenzialen eines DWH, die sich im Rahmen einer Literaturanalyse identifizieren ließen, vgl. Kink/ Höhne/Hess (2008), S. 9.

73 Aus Gluchowski/Kemper (2006), S. 14.

$74 \mathrm{Zu}$ den einzelnen Phasen und Teilschritten des ETL-Prozesses vgl. Gluchowski/Gabriel/ Dittmar (2007), S. 133ff.; Kemper/Mehanna/Unger (2004), S. 23ff.; Bange (2004), S. $85 \mathrm{ff}$; Stock/Büttner (2004), S. $1067 f$. 
zess. Da die Daten der Vorsysteme typischerweise in sehr heterogener Form vorliegen, muss das ETL-Werkzeug den Zugriff auf alle Daten aus den unterschiedlichsten Vorsystemen ermöglichen. Die Transformation als zentrale Aufgabe des ETL-Prozesses bezeichnet die Umwandlung der Ausgangsdaten in das DWH-Zielformat. Weiterhin lässt sich der Transformationsprozess in die Teilschritte der Filterung, Harmonisierung, Aggregation und Anreicherung aufgliedern.

Schließlich werden die extrahierten und transformierten Daten in das DWH geladen und dauerhaft abgelegt. Um eine zeitnahe Bereitstellung der Daten zu gewährleisten, ist in diesem Zusammenhang zu klären, in welchen Zeitintervallen die Daten der operativen Systeme in das DWH geladen werden müssen, um eine ausreichende Aktualität und Qualität der Informationsversorgung zu ermöglichen. Je nach Anwendungsbereich lassen sich die aus den Vorsystemen stammenden und im Vorfeld ausgewählten Daten beispielsweise einmal pro Monat, einmal pro Woche oder auch täglich transferieren.

Einer der zentralen Erfolgsfaktoren für die Gestaltung einer BI-Anwendung ist die Modellierung einer geeigneten multidimensionalen Datenstruktur. ${ }^{75}$ Als Bindeglied zwischen den Datenquellen und den Benutzern dient das Datenmodell zur Realisierung der ETL-Prozesse. ${ }^{76}$ Beim Modellierungsprozess liegt eine der wesentlichen Aufgaben in der Identifizierung relevanter Dimensionen und Fakten. Dimensionen stellen qualitative Informationsobjekte dar, die dazu dienen, die Variablen bzw. Kennzahlen zu beschreiben und auszuwerten. ${ }^{77}$ I. d. R. kommt bei einer DWH-Anwendung eine Reihe von Dimensionen zum Einsatz. ${ }^{78}$ Mit Hilfe von Dimensionshierarchien lassen sich die Kennzahlen aggregieren bzw. disaggregieren. ${ }^{79}$ Kennzahlen stehen für die quantitativen Werte, die sich unter betriebswirtschaftlichen Gesichtspunkten auswerten lassen. ${ }^{80}$ Werden die Kennzahlen in einer sachlich sinnvollen Beziehung zueinander gesetzt, so ergeben sich daraus Kennzahlensysteme. ${ }^{81}$ Die Implementierung mul-

Vgl. Hahn (2003), S. 606.

Vgl. Bange (2004), S. 95. Datenmodelle beschreiben den zu modellierenden Realitätsausschnitt in formaler Form. Ein Datenmodell liefert Informationen über die Struktur der Daten, die Beziehungen zwischen und innerhalb der Datenobjekte, Wertebereiche sowie Integritätsbedingungen. Vgl. Pernul/Unland (2003), S. 10.

Vgl. Hettler/Preuss/Niedereichholz (2003), S. 97.

Um die Multidimensionalität des Datenraums bildhaft darzustellen, hat sich in der Literatur die Metapher eines Datenwürfels bzw. Daten-Cubus etabliert.

Vgl. Becker/Knackstedt (2004), S. 40.

Vgl. Hettler/Preuss/Niedereichholz (2003), S. 97.

Vgl. Liebetruth/Otto (2006), S. 13. 
tidimensionaler Datenstrukturen ist sowohl mit relationalen als auch multidimensionalen Datenbanksystemen möglich. 82

Auf der obersten Ebene der Referenzarchitektur befinden sich alle Methoden und Werkzeuge, mit deren Hilfe sich der multidimensional abgebildete und konsolidierte Datenbestand, der sich infolge der physischen Aufbereitung ergibt, auswerten lässt. Unter den verschiedenen Möglichkeiten zur Datenauswertung sowie zur Präsentation der Berichte spielen das Online Analytical Processing (OLAP) sowie das Data Mining eine große Rolle. ${ }^{83}$ Unter dem Akronym OLAP verbirgt sich eine Softwaretechnologie, die es den jeweiligen Entscheidungsträgern ermöglicht, schnelle, interaktive und vielfältige Zugriffe auf die relevanten, im multidimensionalen Datenmodell abgelegten Daten durchzuführen. ${ }^{84}$ Während die ,klassischen“ OLAP-Operationen Slicing, Dicing, DrillDown und Roll-Up tendenziell vergangenheitsorientierte Analysen ermöglichen, ${ }^{85}$ liegt der Fokus von Data Mining auf der prospektiven Auswertung eines Datenbestands. Durch den Einsatz diverser Algorithmen und Methoden wird das Ziel verfolgt, in einem aufbereiteten Datenbestand interessante Muster zu erkennen, die für die Beantwortung einer konkreten Frage- bzw. Problemstellung relevant sein können.86 Im Gegensatz zur OLAP-Technologie, die die Überprüfung von Hypothesen unterstützt, ist das Data Mining-Konzept darauf ausgerichtet, möglichst selbständig Hypothesen auf Basis der identifizierten Muster zu formulieren. 87

\subsubsection{Ausprägungen von Berichtssystemen}

Wie KEMPER/MEHANNA/UngER bemerken, zeichnen sich Berichtssysteme unter allen Analysesystemen, die sich als BI-Systeme auffassen lassen, durch die

82 Vgl. Gabriel/Röhrs (2003), S. 371. Zur logischen Modellierung einer multidimensionalen Datenstruktur mit Hilfe von Relationen lassen sich verschiedene Varianten nutzen. $\mathrm{Zu}$ den gebräuchlichsten zählen das Star-Schema (Stern-Schema), das Snowflake-Schema (Schneeflocken-Schema) und das Fact-Constellation-Schema. Vgl. Becker/Knackstedt (2004), S. 41. Zu den Eigenschaften und Unterschieden dieser Modellierungsvarianten vgl. Gabriel/Röhrs (2003), S. 371 ff.; Frosch-Wilke (2003), S. 599f. Vgl. Schwarz et al. (2003), S. 196. Zu den Grundlagen des Data Mining und OLAP vgl. Petersohn (2005), S. 48ff.

84 Vgl. Gabriel/Röhrs (2003), S. 350.

$85 \mathrm{Zu}$ den gebräuchlichen OLAP-Operationen vgl. Kemper/Mehanna/Unger (2004), S. 95ff.; Gluchowski/Gabriel/Dittmar (2007), S. 148ff. und 171f.; Oehler (2006), S. 27f.; Jarke et al. (2000), S. 90.

86 Vgl. Gabriel/Röhrs (2003), S. 344f.

87 Vgl. Schwarz et al. (2003), S. 196. 
größte Erscheinungsvielfalt aus. ${ }^{88}$ Einen Vorschlag zur Klassifizierung der verschiedenen Ausprägungen eines Berichtssystems liefert Abbildung 5.89

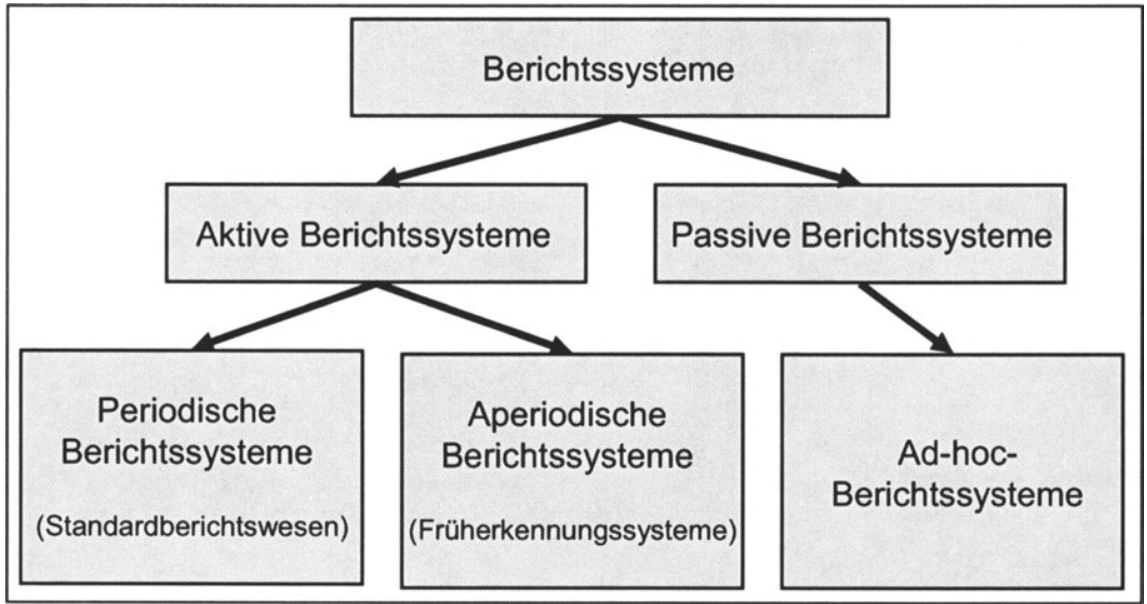

Abbildung 5: Übersicht zur Einordnung von Berichtssystemen 90

Auf der obersten Ebene lassen sich Berichtssysteme nach dem auslösenden Moment einer Berichtserstellung systematisieren.91 Hierbei stehen den aktiven Berichtssystemen die passiven gegenüber. ${ }^{92}$ Während bei aktiven Berichtssystemen die Erstellung und Veröffentlichung der Berichte durch die betriebenen IS ausgelöst wird, fordern passive Berichtssysteme ihre Nutzer dazu auf, selbständig auf den zugrunde liegenden Informationsbestand zuzugreifen und Berichte zu erstellen.

Vgl. Kemper/Mehanna/Unger (2004), S. 111. Mit Blick auf die von KEMPER/MEHANNA/ UNGER präsentierte Übersicht zur Einordnung von Analysesystemen für das Management lassen sich Berichtssysteme als Ausprägung eines generischen BI-Basissystems auffassen. Zusammen mit den konzeptorientierten Systemen bilden die generischen Basissysteme die beiden Kategorien eines BI-Systems. $\mathrm{Zu}$ diesen beiden Kategorien vgl. Kemper/Mehanna/Unger (2004), S. 83f.

HANSEN/NEUMANN präsentieren einen alternativen Ansatz zur Systematisierung von Berichtssystemen. Vgl. Hansen/Neumann (2005), S. 777ff. In Anlehnung an Gluchowski (1998), S. 1178.

91 Der hier gewählte Einstiegspunkt zur Systematisierung von Berichtssystemen geht damit mit der Erkenntnis GÖPFERTS einher, nach deren Auffassung sich die Unterscheidung nach der Erscheinungsweise eines Reports sowie nach dem auslösenden Ereignis der Berichtserstellung zur Einteilung der verschiedenen Berichtsarten durchgesetzt hat. Vgl. Göpfert (2002), S. 148.

Vgl. Gluchowski (1998), S. 1178. 
Charakteristisch für aktive Berichtssysteme ist, dass die Berichte nach einem bestimmten, im Vorfeld zu definierenden Muster erstellt und dem jeweiligen Empfänger(-kreis) präsentiert werden. ${ }^{93}$ Hierzu sind im Vorfeld als quasi einmaliger Aufwand die relevanten Berichtsinhalte, der strukturelle Aufbau sowie die graphische Darstellung der Reports zu definieren. Auf einer feineren Stufe werden innerhalb der Kategorie der aktiven Berichtssysteme die periodischen von den aperiodischen Berichtssystemen unterschieden.

Periodische Berichtssysteme lassen sich für die Zwecke eines Standardberichtswesens einsetzen. Charakteristisch für ein derartiges Berichtssystem ist, dass den Berichtsempfängern die für sie relevanten Informationen in Form standardisierter Berichte zur Verfügung stehen. ${ }^{94}$ Die Berichte werden auf Basis der im Vorfeld definierten Berichtsinhalte, Struktur sowie graphischen Darstellung in einem zyklischen Bereitstellungsprozess oder im Rahmen einer bedarfsorientierten Anfrage erstellt bzw. veröffentlicht.95 Die Veröffentlichung der Reports erfolgt i. d. R. in festgelegten Zeitabständen. Vor dem Hintergrund eines langfristig sich nur geringfügig ändernden Informationsbedarfs der Berichtsempfänger ist das Standardberichtswesen folglich auf eine kontinuierliche sowie stabile Informationsversorgung ausgerichtet.

Neben einer starren Struktur können die Reports eines Standardberichtswesens einen variablen Aufbau aufweisen, um beispielsweise Ausnahmemeldungen, Abweichungen sowie eingetretene Unter- und/oder Überschreitungen vorgegebener Schwellenwerte anzuzeigen. ${ }^{96}$ Für eine anschauliche Präsentation der Berichte lassen sich Tabellen, Grafiken wie z. B. Torten- oder Balkendiagramme sowie geografische Abbildungen beispielsweise in Form einer Landkarte verwenden. ${ }^{97}$

Während periodische Berichtssysteme in festen bzw. regelmäßigen Zeitabständen Berichte erstellen, wird bei aperiodischen Berichtssystemen ein Report nach dem Eintreffen einer besonderen Datenkonstellation - beispielsweise im Fall einer Abweichung von Normwerten - generiert. ${ }^{98}$ Derartige Berichtssysteme werden auch als Früherkennungssysteme oder indikatorbasierte Frühwarnsysteme bezeichnet. ${ }^{99}$ Der Einsatz von Früherkennungssystemen ermöglicht die Implementierung eines Ausnahmeberichtswesens, das auch als Exception Reporting bezeichnet wird. In diesem Zusammenhang dienen Agenten und andere regelgesteuerte Mechanismen dazu, nach Eintritt eines bestimmten Ereignisses

Vgl. dazu Gluchowski/Gabriel/Chamoni (1997), S. 48f.

94 Ein Beispiel für den Einsatz eines Standardberichtswesens in einem Lebensmittelfilialbetrieb liefern Hansen/Neumann (2005), S. 780.

Vgl. Bange (2004), S. 94.

96 Vgl. Gluchowski (1998), S. 1178.

97 Vgl. Bange (2004), S. 95.

98 Vgl. dazu Gluchowski/Gabriel/Chamoni (1997), S. 48.

99 Vgl. Gluchowski (1998), S. 1178; Gluchowski/Gabriel/Chamoni (1997), S. 48f. Alexander Pastwa - 978-3-631-75488-7 
dem vorgesehenen Empfänger(-kreis) entsprechende Berichte zur Verfügung zu stellen. Als derartige Ereignisse kommen beispielsweise ein bestimmter Zeitpunkt, die Verfügbarkeit von Daten für die Berichtserstellung sowie die Aufnahme bestimmter Werte in eine Datenbank in Frage. 100

Im Gegensatz zu einem IT-gestützten Standardberichtswesen wird durch Früherkennungssysteme gewährleistet, dass wichtige Entwicklungen, die im Rahmen eines Standardberichtswesens erst zu einem späteren Zeitpunkt oder möglicherweise gar nicht erkannt würden, durch eine automatische Benachrichtigung des Systems - beispielsweise per Mail - aufgedeckt werden, ${ }^{101}$ so dass die Möglichkeit einer zeitnahen Einflussnahme des betrieblichen Entscheidungsträgers auf die Unternehmungsentwicklung gewahrt werden kann.

In Abgrenzung zu den aktiven Berichtssystemen zeichnen sich passive Berichtssysteme dadurch aus, dass sie nicht selbständig Berichte generieren, sondern auf die Anfrage des Benutzers warten. ${ }^{102}$ Mit Blick auf die Passivität des Berichtssystems bzw. die aktive Rolle Berichterstellers lässt sich ein solches System auch als Abfrage- oder Auskunftssystem bezeichnen. ${ }^{103}$ Als Ausprägung eines passiven Berichtssystems haben sich so genannte Ad-hocBerichtssysteme etabliert. ${ }^{104}$ In Abgrenzung zum Standardberichtswesen fokussieren Ad-hoc-Berichtssysteme auf den spontanen bzw. unregelmäßigen Zugriff auf die Daten der Unternehmung, in denen die relevanten Berichtsinformationen vorliegen. Als Ergebnis einer Datenabfrage können die generierten Berichte dabei in einer starren oder variablen Struktur angezeigt werden. 105

I. d. R. unterliegt der Informationsbedarf der Berichtsempfänger, die ein Adhoc-Berichtssystem nutzen, stärkeren Schwankungen bzw. Änderungen, so dass ein höheres $\mathrm{Maß}$ an Flexibilität bei dem Zugriff auf die Informationen zu fordern ist. Für die Erstellung der Berichte lassen sich verschiedene Berichtswerkzeuge einsetzen, die wiederum auf verschiedene Datenquellen zugreifen können. Zwischen diese beiden Komponenten wird ein Datenmodell geschaltet, um den zu berichtenden Realitätsausschnitt auf einer semantischen sowie logischen Ebene abzubilden. ${ }^{106}$ Mit Blick auf den Eigenanteil des Informationsempfängers bei der Berichterstellung sind von diesem IT-Kenntnisse zu erwarten. 107 Kommt zur Implementierung eines Ad-hoc-Systems ein Datenbanksystem zum Einsatz, muss der Benutzer zur Erzielung eines gewünschten Abfrageergebnis-

Vgl. Bange (2004), S. 95.

Vgl. Kemper/Mehanna/Unger (2004), S. 111 f.

102 Vgl. Gluchowski/Gabriel/Chamoni (1997), S. 49f.; Kemper/Mehanna/Unger (2004), S. 112.

Vgl. Gluchowski (1998), S. 1178.

Vgl. Kemper/Mehanna/Unger (2004), S. 112.

Vgl. Gluchowski (1998), S. 1178.

Vgl. Bange (2004), S. 95.

Vgl. Kemper/Mehanna/Unger (2004), S. 112. 
ses beispielsweise Kenntnisse der Datenstrukturen besitzen. 108 Für die Erstellung der Berichte sowie für die Implementierung eines leistungsfähigen $\mathrm{Be}$ richtssystems sind folglich hohe Anforderungen zu erfüllen. ${ }^{109}$

\subsubsection{Kernprozesse des betrieblichen Berichtswesens}

Der vorliegende Abschnitt verfolgt das Ziel, die Kernprozesse des betrieblichen Berichtswesens zu präsentieren. Unter Einbezug der Beiträge von LEßWENG und KeMPER/MehanNa/Unger, die sich neben GÖPFERT mit den relevanten Prozessen im Berichtswesen beschäftigt haben, 110 lassen sich mit der Informationsbeschaffung, Berichtsproduktion, Berichtsdistribution und Berichtsaufnahme vier Kernprozesse des Reportings identifizieren. Die Kernprozesse fokussieren die Verarbeitung der Geschäfts- und Finanzinformationen und stellen gleichsam die vier Teilprozesse eines Berichtsprozesses dar. Abbildung 6 verdeutlicht die Anordnung der Prozesse.

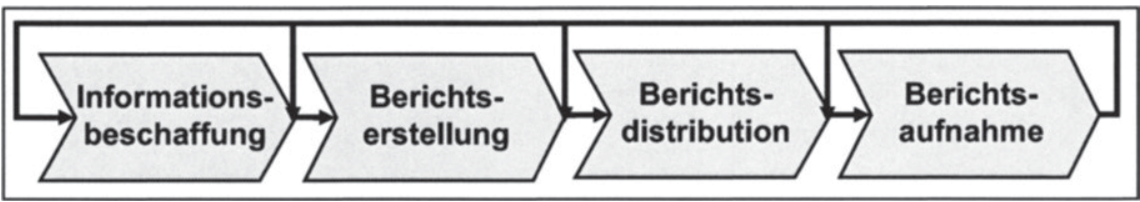

Abbildung 6: Phasen des Berichtsprozesses

\subsubsection{Informationsbeschaffung}

Der Teilprozess Informationsbeschaffung umfasst Aktivitäten, die zum Ziel haben, alle Daten und Informationen, die zur Berichtserstellung erforderlich sind, zu identifizieren, in den betreffenden IS zusammenzuführen, dort dauerhaft abzulegen und zu verwalten. Als Daten- und Informationsquellen kommen zum einen unternehmungsinterne Systeme in Frage, die die zu beschaffenden Informationen entweder in einer strukturierten oder unstrukturierten Form vorhalten. Unstrukturierte bzw. schwach strukturierte Informationen zeichnen sich durch eine geringe gemeinsame innere Struktur aus. ${ }^{111}$ In einem erweiterten Verständ-

108 Vgl. Gluchowski (1998), S. 1178.

109 Eine Zusammenfassung der Grundsätze bzw. Gestaltungsregelen, die für die Implementierung eines Berichtssystems einzuhalten sind, liefern BLOHM und GÖPFERT. Vgl. Blohm (1980), S. 319; Göpfert (2002), S. 152.

110 Vgl. Leßweng (2003a), S. 335ff.; Leßweng (2003b), S. 86ff.; Leßweng (2004), S. 42; Kemper/Mehanna/Unger (2004), S. 110f.; Göpfert (2002), S. 144ff.

111 Vgl. Bange (2004), S. 17. 
nis eignen sich neben Textdaten, die beispielsweise in Internetseiten, E-Mails oder abgespeicherten Berichten enthalten sind, auch Bilder, Tonaufzeichnungen und Videos als Quellen unstrukturierter Informationen. Da die für die Berichtsproduktion interessanten Inhalte auch von außerhalb der Unternehmung stammen können, ${ }^{112}$ sind auch unternehmungsexterne Quellen bei der Informationsbeschaffung zu berücksichtigen.

Als datenhaltende Systeme zur Verwaltung strukturierter Daten werden Datenbanksysteme eingesetzt, die sich sowohl für die Verarbeitung operativer transaktionsorientierter Daten als auch für analyseorientierte Zwecke z. B. im Rahmen von Berichtssystemen eignen.113 Wird die Verarbeitung von unstrukturierten Informationen angestrebt, lassen sich Dokumentenmanagementsysteme (DMS) nutzen. Derartige IS werden mit dem Ziel eingesetzt, elektronische Dokumente im Rahmen von organisatorischen Prozessen zu speichern, zu verwalten, zu archivieren und über geeignete Suchkriterien den Anwendern wieder zugänglich zu machen. 114

Kommt ein DWH-gestütztes Berichtssystem zum Einsatz, fokussiert der Teilprozess Informationsbeschaffung nicht nur den manuellen Import der berichtsrelevanten Daten und Informationen in die Vorsysteme einer derartigen Applikation. Unter Informationsbeschaffung wird darüber hinaus auch die Durchführung eines ETL-Prozesses verstanden, mit dessen Hilfe die berichtsrelevanten Daten und Informationen aus den Vorsystemen extrahiert, transformiert und in das DWH geladen werden.

\subsubsection{Berichtserstellung}

Dem Kernprozess der Informationsbeschaffung schließt sich die Berichtserstellung an. Gegenstand dieses Prozesses ist es, aus den gespeicherten Daten einen formalisierten Bericht zu erstellen. Für die Informationserzeugung lassen sich Methoden- und Modelldatenbanken nutzen, die eine bessere Auswertung der Informationen ermöglichen. ${ }^{115}$ Neben OLAP- und Data Mining-Tools lassen sich

112 Vgl. Leßweng (2004), S. 41.

113 Vgl. Abschnitt 2.2. Auf die Bedeutung von Datenbanksystemen als Rückgrat betrieblicher Datenverarbeitung verweisen z. B. Gabriel/Röhrs (2003), S. 357. Eine Übersicht zu den Einsatzmöglichkeiten von Datenbanksystemen in verschiedenen Branchen, Institutionen und Funktionsbereichen liefern Gabriel/Röhrs (1995), S. 8ff.

$114 \mathrm{Zu}$ den Grundlagen und Unterstützungspotenzialen von DMS vgl. Thome/Böhn/Hagn (2003), S. 914ff.; Dittmar (2004), S. 291f.; Lehner (2000), S. 339. Als elektronisches Dokument wird jede Art von unstrukturierten und strukturierten Informationen verstanden, die als Einheit vorliegen und in einem IS gespeichert sind. Vgl. Lehner (2000), S. 339f.

115 Vgl. Göpfert (2002), S. 145. 
spezialisierte Werkzeuge zur Abfrage und Berichtserstellung nutzen. ${ }^{116}$ Neben einfachen Abfragewerkzeugen, mit denen unter Einsatz grafischer Bedienelemente Datenextrakte gebildet werden können, lassen sich Berichtsgeneratoren einsetzen. Berichtsgeneratoren zeichnen sich durch eine größere Funktionalität aus, indem sie zusätzlich zur inhaltlichen und optischen Anreicherung Gestaltungs- und Berechnungsoptionen zur Verfügung stellen. So genannte Managed Query Environments (MQE) lassen sich zum Aufbau und Betrieb eines unternehmungsweiten Reportings nutzen. $\mathrm{Zu}$ den bereitgestellten Features von MQETools gehören z. B. eine Administrationsfunktion oder die Bereitstellung einer semantischen Schicht, die die Zuordnung von vertrauten Begriffen aus der Sprachwelt der Endanwender zu konkreten Datenobjekten ermöglicht. ${ }^{117}$

Welche Elemente eines Reports sowie zwischen diesen existierende Beziehungsstrukturen bei der Berichtserstellung zu beachten sind, lässt sich anhand eines Berichtsmodells verdeutlichen, das in der Abbildung 7 dargestellt ist. 118 Ziel des in dem Berichtsmodell abgebildeten Pfades ist es, aus einer abstrakten Berichtsvorstellung heraus eine konkrete Berichtsinstanz zu erstellen, die dem Informationsbedarf eines Berichtsempfängers oder einer Berichtsempfängergruppe genügt.

Wie in Abbildung 7 dargestellt ist, zeichnet sich ein abstrakter Bericht zum einen durch den Informationsbedarf (linker Ast), zum anderen durch eine formale Struktur (rechter Ast) aus. ${ }^{119}$ Die formale Struktur eines Berichts ist einerseits durch den Aufbau der Berichtsinhalte andererseits durch die Darstellungsform bzw. das Layout gekennzeichnet, die je nach Präferenz des Berichtsempfängers eine andere Ausprägung annehmen können. Beim Aufbau, d. h. bei der strukturellen Anordnung der Berichtsinhalte, ist Sorge zu tragen, dass die Übersichtlichkeit und Verständlichkeit der berichteten Informationen gewahrt bleiben. KÜPPER empfiehlt in diesem Zusammenhang die Verwendung gleicher Gliederungsprinzipien sowie die gleichförmige Gestaltung zentraler Berichtselemente, um eine einheitliche Berichtsstruktur zu gewährleisten. ${ }^{120}$

Die Festlegung der Darstellungsform sorgt bei der Generierung eines Reports dafür, dass die nachgefragten Informationen nach den Vorstellungen des Empfängerkreises in dem gewünschten Erscheinungsbild vorliegen. Für die Darstellung bzw. das Layout der zu berichtenden Informationen stehen drei Varianten zur Verfügung. Den rein textuell aufbereiteten Berichtsinformationen, die sich mit Hilfe von Tabellen sowie ausformulierten Texten darstellen lassen, stehen

Vgl. Gluchowski (1998), S. 1180ff.

117 Als Datenobjekte kommen z. B. Datenbanktabellen oder Tabellenattribute in Frage.

118 Die Ausführungen orientieren sich an dem Vorschlag von BECKER/KÖSTER/SANDMANN, die ein entsprechendes Modell vorgelegt haben. Vgl. Becker/Köster/Sandmann (2006), S. 504.

$119 \mathrm{Vgl}$. dazu auch Kemper/Mehanna/Unger (2004), S. 110.

120 Vgl. Küpper (1997), S. 155. 
diejenigen Inhalte gegenüber, die sich grafischer Abbildungen wie beispielsweise Kreis-, Balken- oder Säulendarstellungen bedienen. ${ }^{121}$ Als dritte Alternative kommt eine Mischform in Frage, die zur Visualisierung der Inhalte sowohl textuelle als auch grafische Darstellungselemente verwendet. Mit Blick auf das zum Einsatz kommende Präsentationsmedium lassen sich Berichte einerseits als digitale Dokumente ablegen. Andererseits werden die generierten Berichte auf konventionellem Weg als Papierdokumente ausgedruckt.

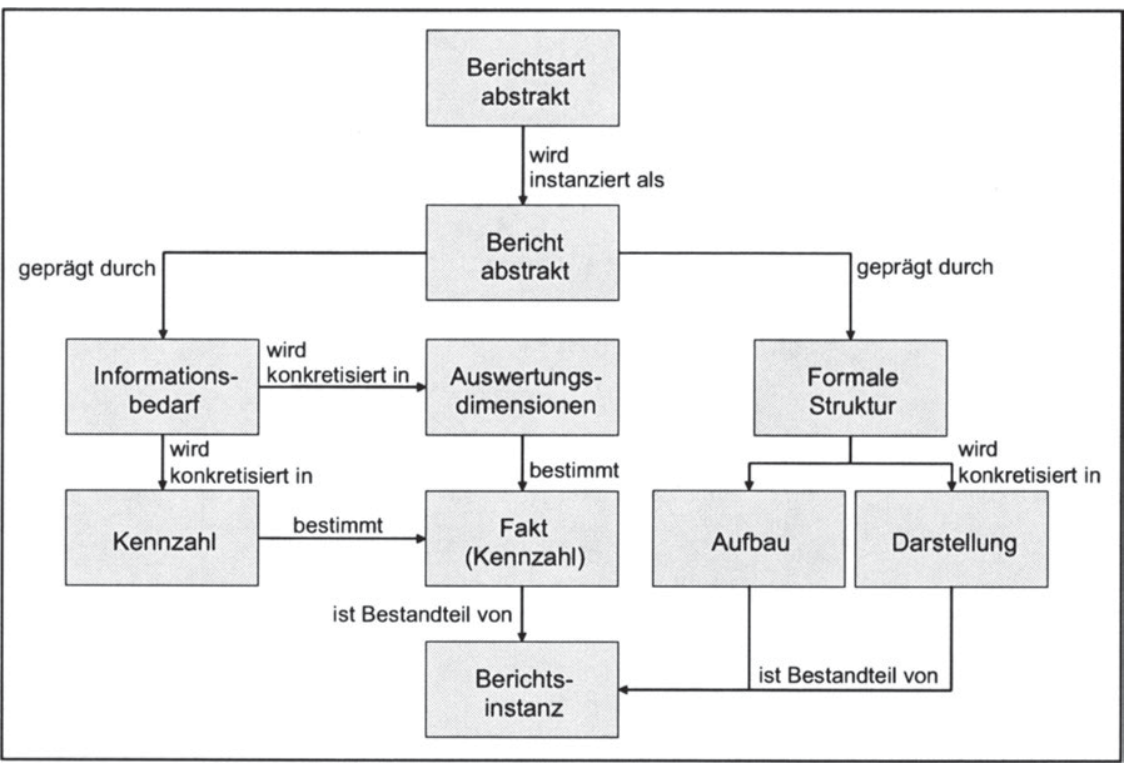

Abbildung 7: Berichtsmodell 122

Die inhaltliche Komponente eines Berichts wird durch den Informationsbedarf bestimmt, der die Nachfrage eines Berichtsempfängers oder einer Berichtsempfängergruppe nach relevanten und korrekten Informationen widerspiegelt. ${ }^{123}$ Der Informationsbedarf eines Berichtsempfänger(-kreises) konkretisiert sich durch die Betrachtungsobjekte bzw. die Dimensionen, die zur Auswertung der Kennzahlen dienen. Durch die Verknüpfung der Kennzahlen mit den sie be-

121 Vgl. Gluchowski (2006), S. 211.

122 Vgl. Becker/Köster/Sandmann (2006), S. 504.

123 Zu den Verfahren, die sich zur Bestimmung des Informationsbedarfs nutzen lassen, vgl. Krause/Schmitz (2006), S. $351 \mathrm{ff}$. 
schreibenden Dimensionen entstehen die zu berichtenden Fakten eines Berichts. ${ }^{124}$

Die inhaltlichen Aussagen der zu berichtenden Informationen lassen sich darüber hinaus im Hinblick auf ihren Informationsgehalt beschreiben. Dabei ist im Vorfeld zu vereinbaren, welcher Sachverhalt im Mittelpunkt der Informationsversorgung stehen soll. Gegenstand eines Berichts können beispielsweise diejenigen Informationen sein, die für die Zwecke des internen und/oder externen Berichtswesens benötigt werden. ${ }^{125}$ Damit die berichteten Informationen einen hohen Aussagehalt enthalten, ist ferner zu entscheiden, in welchem Detaillierungsgrad sowie in welcher Genauigkeit die Informationen vorliegen müssen. Da aggregierte Berichtsinhalte mit Informationsverlusten verbunden sind, 126 ist der Wahl eines geeigneten Aggregationsniveaus, das sich am Informationsbedarf des Berichtsempfängers orientiert, besondere Aufmerksamkeit zu schenken.

Werden die Berichtsinhalte in die aus der abstrakten Berichtsvorstellung heraus vorgegebene formale Struktur überführt, so resultiert daraus die Berichtsinstanz und damit die konkrete Ausprägung eines abstrakten Berichts. Neben der bereits beschriebenen inhaltlichen sowie formalen Komponente zeichnet sich die konkrete Berichtsinstanz dadurch aus, dass in ihr festgelegt ist, welcher Mitarbeiter für die Erstellung der Berichtsinstanz verantwortlich ist. Hierbei ist anzumerken, dass die Verantwortung für den Berichtsinhalt von der technischen Zuständigkeit abweichen kann. Ebenso ist es möglich, dass die berichtserstellende Person nicht identisch mit dem Berichtsverantwortlichen ist, auch wenn diese Arbeitsteilung eher eine Ausnahme im betrieblichen Berichtswesen darstellt. ${ }^{127}$

\subsubsection{Berichtsdistribution}

Als dritter Teilprozess eines Berichtsprozesses lässt sich die Phase der Berichtsdistribution identifizieren. Alternativ lässt sich dieser Prozess auch als $\mathrm{Be}-$ richtsverteilung, Berichtsübermittlung oder Berichtsdiffusion bezeichnen. ${ }^{128}$ Gegenstand dieses Prozesses sind alle Aktivitäten, die die zeitnahe und korrekte Weiterleitung der erstellten Berichte an den im Vorfeld definierten Empfänger(kreis) fokussieren. Auch die Erläuterungen, die zum Verständnis einzelner Berichtsinhalte beitragen, sind mit dem Bericht zu übermitteln. Die Art der Weiter-

124 Vgl. Becker/Köster/Sandmann (2006), S. 504.

125 Zur Unterscheidung in ein internes und externes Berichtswesen vgl. Abschnitt 2.1.2.

126 Vgl. Sorter (1969), S. 14.

127 Vgl. Gluchowski (2006), S. 212.

128 Vgl. Kemper/Mehanna/Unger (2004), S. 111. 
leitung, d. h. der Kommunikationsweg, hängt dabei von der Wahl des Präsentationsmediums ab.

Liegen die Berichte als elektronische Dokumente vor, erfolgt eine Weiterleitung der Reports an die jeweiligen Berichtsempfänger über einen digitalen Kommunikationsweg. Berichte, die als Papierdokumente ausgedruckt werden, lassen sich Berichtsempfängern auf postalischem Weg zukommen oder direkt aushändigen. Die digitale Berichtsdistribution lässt sich nach dem Push-Prinzip gestalten bzw. betreiben. ${ }^{129}$ Beim Push-System erfolgt ein angebotsinduziertes Vorgehen, so dass die Informationen automatisch an den Berichtsempfänger weitergeleitet werden. ${ }^{130}$ Die Erweiterung des Push-Ansatzes wird als Publish and Subscribe-Prinzip bezeichnet. ${ }^{131}$ Ausgehend von einem Informationsbedarfsprofil, das der Anwender erstellen muss, übernimmt das System die aktive Weiterleitung der Informationen. Eine aktive Informationsdiffusion erfolgt z. B. in regelmäßigen Intervallen und/oder bei geänderten Datenkonstellationen, die zuvor über entsprechende Ereignisregeln festgelegt werden müssen. ${ }^{132}$

Um die produzierten Berichte auch zu einem späteren Zeitpunkt aufzurufen und/oder an die betreffenden Rezipienten weiterzuleiten, müssen die Berichtsdokumente bzw. die -dateien kategorisiert bzw. katalogisiert, zwischengespeichert oder dauerhaft abgelegt werden. Daher spielt im Rahmen der Berichtsdistribution auch die Berichtsverwaltung eine große Rolle. Für die Zwecke der Berichtsverwaltung lassen sich beispielsweise Datenbanksysteme oder DMS nutzen. Eine Berichtsverwaltung ist zum einen in den Konstellationen bedeutsam, in denen der Berichtsersteller nicht gleichzeitig auch der Berichtsnutzer ist. Diese Situation ist beispielsweise gegeben, wenn eine ControllingAbteilung mit der Generierung von Berichten beauftragt ist, die für die Geschäftsführung bestimmt sind. Zum anderen stiftet die Zwischenspeicherung von Berichten Nutzenpotenziale, wenn die Berichtsverarbeitung asynchron verläuft, d. h., wenn die Berichtsaufnahme zeitversetzt nach der Berichtsdistribution erfolgt.

\subsubsection{Berichtsaufnahme}

Der Teilprozess der Berichtsaufnahme bildet den vorläufigen Abschluss des Berichtsprozesses. Wird ein Bericht nach dem Push-Prinzip per Mail an die Be-

129 Prinzipiell lässt sich dieses Verfahren auch zur Weiterleitung von Papierdokumenten anwenden. Da die vorliegende Arbeit einen IT-gestützten Berichtsprozess fokussiert, sind vor allem die IT-gestützten Push-Systeme relevant.

130 Die Weiterleitung erfolgt meistens regelbasiert. Ein Beispiel für ein Push-System ist ein aktives Berichtssystem.

131 Vgl. Kurz (1999). S. 482ff.

132 Vgl. Dittmar (2004), S. 300. 
richtsempfänger weitergeleitet, lässt sich der erfolgreiche Eingang des Berichts mit Hilfe einer Empfangsbestätigung quittieren, die ebenfalls per Mail dem Berichtssender zugestellt wird. Eine derartige Empfangsbestätigung lässt sich automatisieren oder manuell auslösen. Da die weitergeleiteten Berichte zwischengespeichert werden können, ist es möglich, dass eine Berichtsaufnahme auch zu einem späteren Zeitpunkt nach Eintreffen des Berichts erfolgt. Eine derartige Konstellation liegt z. B. vor, wenn die Berichtsdokumente per Mail versendet, in einem entsprechenden Berichtsordner auf einem Datei-Server oder in einem DMS abgelegt und erst zu einem späteren Zeitpunkt geöffnet werden.

Werden die Berichte nach ihrer Produktion zunächst beim Berichtsersteller zwischengespeichert, erfolgt eine Benachrichtigung, dass der Report ,zur Abholung“ bereit steht. In diesem Fall ist für die Berichtsaufnahme das PullPrinzip anzuwenden. Während beim Push-Prinzip ein angebotsinduziertes Vorgehen erfolgt, zeichnet sich das Pull-Prinzip dadurch aus, dass der Benutzer einen Zugriffsvorgang auslöst, um aus den zur Verfügung stehenden Informationsquellen die nachgefragten Inhalte zu erhalten. ${ }^{133}$ Im Gegensatz zum PushPrinzip muss der Anwender beim Pull-Verfahren wissen, wo sich die benötigten Informationen befinden, ${ }^{134}$ so dass dem Pull-Verfahren eine nachfrageinduzierte Vorgehensweise zugrunde liegt. Ein solcher Sachverhalt liegt vor, wenn die Berichtsnutzer über eine geeignete Schnittstelle auf einen in einem DMS vorgehaltenen Report zugreifen, entweder den gesamten Bericht oder nur einen Auszug des Berichts kopieren und die Kopie auf ihrem lokalen System als separates Dokument abspeichern.

Im Idealfall stimmen die Berichtsinhalte mit dem im Vorfeld definierten Informationsbedarf überein. Wie in Abbildung 6 verdeutlicht ist, existieren zwischen den Teilprozessen Rücksprünge. Ein Rücksprung vom Teilprozess der Berichtsaufnahme zum Teilprozess der Berichtserstellung ist $\mathrm{z}$. B. erforderlich, wenn ein Bericht nicht den Anforderungen des Berichtsempfängers entspricht, so dass eine Überarbeitung des Berichts notwendig ist. Ursachen für die NichtErfüllung der Anforderungen des Berichtsempfängers lassen sich beispielsweise in inhaltlichen Divergenzen identifizieren, die dadurch entstehen, dass die in einem Bericht enthaltenen Informationen nicht dem Informationsbedarf des Berichtsnutzers entsprechen. Eine weitere Ursache ist in einer fehlerhaften Datenübertragung zu sehen.

Die Identifizierung der Kernprozesse im Berichtswesen erfolgte mit Blick auf die grundsätzliche Möglichkeit zur Gestaltung aller Reporting-Aktivitäten, die die effiziente und effektive Transformation der zu berichtenden Geschäfts- und Finanzinformationen zum Gegenstand haben. Diese Einschränkung führt dazu, dass die von LEBWENG thematisierte, nach der Berichtsaufnahme einsetzende

133 Eine Ausprägung eines Pull-Systems ist z. B. ein passives Berichtssystem.

134 Vgl. Lehner (2000), S. 334. 
Phase der Berichtsdiskussion nicht als Kernprozess des Berichtswesens gewertet wird. ${ }^{135}$ Während der Berichtsdiskussion werden die berichteten Inhalte mit weiteren Berichtsempfängern und Entscheidern erörtert mit dem Ziel, den Erkenntnisstand der beteiligten Akteure zu erweitern, Entscheidungen vorzubereiten und zu kontrollieren sowie Aktionen auszulösen.136 $\mathrm{Da}$ es sich hierbei in erster Linie um eine kommunikative sowie kognitive Leistung der Berichtsempfänger handelt, die auf die Interpretation und nicht auf den Fluss sowie die Transformation der Informationen ausgerichtet ist, wird die Berichtsdiskussion in der vorliegenden Arbeit nicht als Kernprozess im Berichtswesen aufgefasst.

\subsection{Defizite in den Kernprozessen des Berichtswesens}

Nachdem in den vorangehenden Ausführungen sowohl die begrifflichen und konzeptionellen Grundlagen als auch die Möglichkeiten einer informationstechnischen Unterstützung des Berichtswesens thematisiert wurden, hat der vorliegende Abschnitt zum Ziel, Defizite aufzuzeigen, die der Forderung entgegenstehen, ein Reporting zu betreiben, das zukunftsorientierte, entscheidungsrelevante und international vergleichbare Informationen zur Verfügung stellt. 137 Den Vorschlag von LEßWENG aufgreifend, lassen sich unter dem Oberbegriff Defizit sowohl Störungen bzw. Störungsursachen als auch Probleme und Schwachstellen verstehen, ${ }^{138}$ wobei Probleme und Schwachstellen in Folge von Störungen auftreten. Die in Abbildung 8 dargestellte Übersicht dient zur Systematisierung der Defizite.

$\mathrm{Da}$ bei der Ausführung von Berichtsprozessen die Verarbeitung von Informationen im Vordergrund steht, lassen sich zur Gruppierung der Störungsursachen die drei Perspektiven der Semiotik heranziehen. ${ }^{139}$ Folglich können zweckbezogene, inhaltsbezogene sowie syntaxbezogene Defizite identifiziert werden. Die Störungsursachen wirken sich zum einen auf die Effizienz, zum anderen auf

Vgl. Leßweng (2003a), S. 336.

Siehe dazu die Berichtszwecke in Abschnitt 2.1.3.

137 Vgl. Böcking (1998), S. 18ff. BECKER/SEIDEL/JANIESCH formulieren in prägnanterer Form, dass das Berichtswesen mit der Problematik konfrontiert ist, dass den Managern nicht die Informationen für bestmögliche Entscheidungen zur Verfügung stehen. Vgl. Becker/Seidel/Janiesch (2008), S. 229.

138 Vgl. Leßweng (2003), S. 98.

139 Damit folgt die vorliegende Arbeit dem Vorschlag von Leßweng (2003b), S. 184ff. Zur Semiotik vgl. Fußnote 14. 
die Effektivität des Reportings aus. ${ }^{140}$ Der dieser Arbeit zugrunde liegende Effizienz- und Effektivitätsbegriff orientiert sich an dem von KÜNKELE/SCHÄFFER im Zusammenhang mit der Budgetkontrolle geprägten Verständnis dieser beiden ökonomischen Zielgrößen. ${ }^{141}$ Diesem Verständnis folgend, setzt sich die Effektivität des Reportings mit der Frage auseinander, inwieweit der Output beim Berichtswesen, d. h. die konkreten Berichte, dem im Vorfeld definierten Informationsbedarf des Berichtsempfängers entspricht und zu einer zielgerichteten Entscheidungsunterstützung beiträgt. Die Effizienz der Informationsverarbeitung fokussiert auf die Ressourcen, die für die Erstellung, Auswertung sowie Interpretation der Berichtsinformationen aufgewendet werden müssen. Eine effiziente Informationsverarbeitung liegt folglich vor, wenn die Verarbeitung der Geschäfts- und Finanzinformationen mit minimalen Ressourcen erzielt wird. Der wichtigste Kostenblock, der sich negativ auf die Effizienz des Reportings auswirkt, sind die Personalkosten, d. h. die Kosten, die dadurch entstehen, dass Personen entweder auf der Empfänger- oder auf der Erstellerseite mit der Verarbeitung der Berichte beschäftigt sind.142 Darüber hinaus verdeutlicht Abbildung 8, welche Kernprozesse des Berichtswesens von dem jeweiligen Defizit am stärksten betroffen sind.

Abschnitt 2.3.1 widmet sich zunächst den zweckbezogenen Störungen, während Abschnitt 2.3.2 zu den inhaltsbezogenen Defiziten Stellung nimmt. ${ }^{143} \mathrm{Ab}-$ schnitt 2.3.3 thematisiert syntaxbezogene Defizite, die technisch und/oder Personen bedingt sein können.

140 Generell steht die Effektivität in der Betriebswirtschaftslehre als Grad für die Zielerreichung und lässt sich folglich als Maß für die Arbeitsleistung bzw. den Output auffassen. Vgl. Poluha (2007), S. 74. Die Bedeutung der Effektivität wird in der plakativen Aussage „doing the right things“ deutlich. Vgl. z. B. Werner (2002), S. 10. Während die Effektivität darauf abzielt, die richtigen Dinge zu tun, geht es bei der Effizienz darum, die Dinge, die getan werden müssen, richtig zu tun (,doing the things right"). Als Unterziel der Effektivität fokussiert die Effizienz die Ressourcenwirtschaftlichkeit. Zur Gegenüberstellung der beiden Begriffe vgl. Poluha (2007), S. 74; Oestereich/Weiss (2008), S. 419.

141 Vgl. Künkele/Schäffer (2007), S. 76.

142 Nach Schmelz lagen die Kosten für das Reporting zu Beginn der 90er bei über $30 \%$ des Informationsverarbeitungsbudgets. Vgl. Schmelz (1995), S. 72.

143 Auf die zunehmende Bedeutung der Benutzer- und Empfängerorientierung bei der Unternehmensberichterstattung deutet z. B. Horváth (2006), S. 602. 


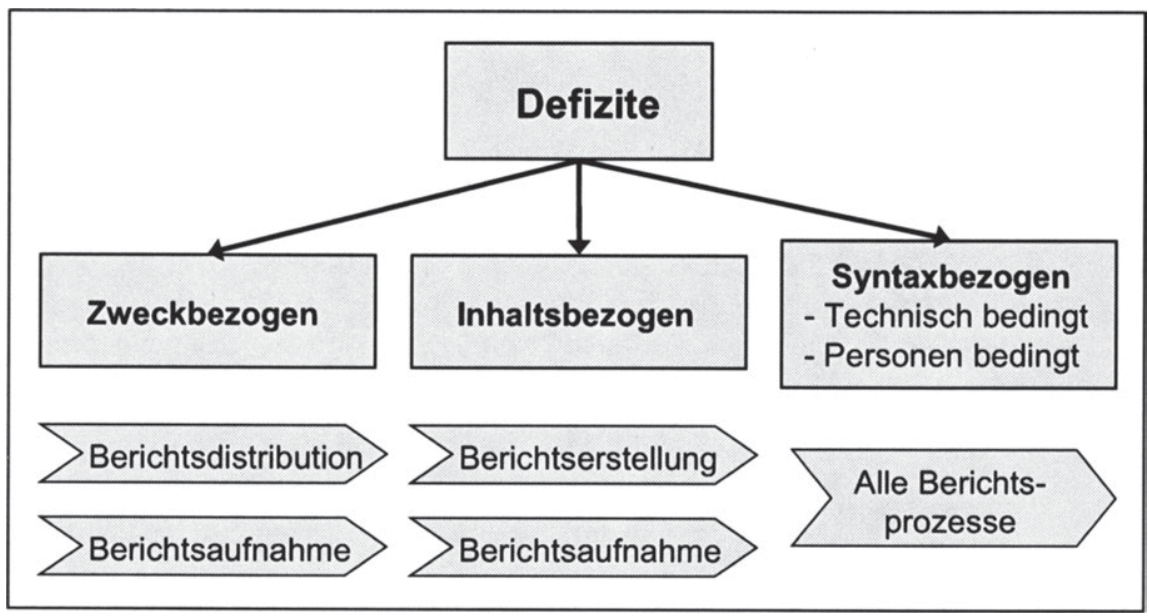

Abbildung 8: Übersicht zu den Problemfeldern bei der Informationsverarbeitung im betrieblichen Berichtswesen

\subsubsection{Zweckbezogene Defizite für die Prozesse „Berichtsdistribution“ und „Berichtsaufnahme“}

Mit der geringen Empfängerorientierung (Abschnitt 2.3.1.1) und der niedrigen Informationsaktualität (Abschnitt 2.3.1.2) lassen sich zwei Defizite identifizieren, die den Zweckbezug des Reportings beeinflussen. Die im Folgenden thematisierten Störungen wirken sich sowohl auf die Berichtsverteilung als auch auf die Berichtsaufnahme aus.

\subsubsection{Geringe Empfängerorientierung}

Unter den verschiedenen Gefährdungspotenzialen, die der Effektivität und Effizienz der Informationsverarbeitung im betrieblichen Berichtswesen entgegenwirken, ist die Störungsursache zu erkennen, dass eine einfache und schnelle Auswertung der in Form eines Berichts bereitgestellten Geschäfts- und Finanzinformationen oftmals verhindert wird. ${ }^{144}$ Als Hauptgründe für eine nicht empfängerorientierte Informationsversorgung lassen sich zum einen eine mangelnde

144 Gestützt wird diese Auffassung auch von BECKER/KÖSTER/SANDMANN, deren Einschätzung zufolge der kritische Engpass für ein erfolgreiches Unternehmensreporting nicht in der Existenz eines auswertbaren Informationsangebots, sondern in der fehlenden Selektion, Aufbereitung und Interpretation der Informationen liegt. Vgl. Becker/Köster/ Sandmann (2006), S. 502. 
Relevanz der Berichtsinhalte, zum anderen eine Überflutung an Informationen identifizieren. ${ }^{145} \mathrm{Da}$ Manager ungefähr die Hälfte ihrer Arbeitszeit ,verschenken“, um Informationen zu finden, die sie benötigen, ${ }^{146}$ stellt der schnelle Zugriff auf relevante Informationen eine wichtige Anforderung dar, damit Entscheidungsträger ihre Aufgaben erfüllen können. ${ }^{147}$

Mit Blick auf die Gefahr der Informationsüberflutung (Information Overload) bzw. des Zahlenfriedhofs ist zu konstatieren, 148 dass durch die Vielfalt und die Menge der berichteten Informationen eine schnelle kognitive Verarbeitung dieser von den betrieblichen Fach- und Führungskräften nicht geleistet werden kann.149 Die Anforderung, Informationen schnell zu verarbeiten, besitzt eine große Bedeutung, da zur betrieblichen Planungs- und Entscheidungsunterstützung vor allem Informationen verarbeitet werden müssen, die unpräzise, vage und verbal formuliert sind. 150

Das Problem der Informationsüberflutung wird dadurch gefördert, dass betriebliche Entscheidungsträger zur Befriedigung ihres individuellen Informationsbedarfs oftmals mit standardisierten, nicht empfängerorientierten Berichten versorgt werden, die sich wiederum zu einer rational nicht mehr auswertbaren Informationsmenge summieren können. ${ }^{151}$ LIEBETRUTH/OTTO kritisieren in diesem Zusammenhang die wachsende Daten- und Kennzahlenflut, zu der insbesondere der Einsatz von DWH-Systemen beiträgt. 152 Verstärkt wird das Problem der Informationsüberflutung durch das so genannte Phänomen der Berichtsremanenz, das besagt, dass einmal erstellte Berichte i. d. R. hinsichtlich ihrer Aussagekraft bzw. Eignung selten hinterfragt, sondern weiterhin produziert und den jeweiligen Empfängern vorgelegt werden.

145 Vgl. Rechkemmer (1998), S. 380.

146 Vgl. Besemer (2008), S. 8.

147 Auf das Problem, dass wichtige Informationen nicht gebündelt in einem Bericht dargestellt, sondern auf mehreren Seiten verteilt werden, verweist Willis (2003), S. 56. Vgl. Mertens (1999), S. 414; Krause/Schmitz (2006), S. 351.

149 Vgl. Becker/Seidel/Janiesch (2008), S. 229; Volnhals/Hirsch (2008), S. 50. Wie GABAIX ET AL. bemerken, gehen jeder Entscheidungsfindung die Akquisition sowie die Verarbeitung von Informationen voraus. Vgl. Gabaix et al. (2006), S. 1042. Zur Diskussion verschiedener Auslegungen des Terminus „Information Overload“"vgl. Volnhals/Hirsch (2008), S. 51.

150 Vgl. Werners (1994), S. 1.

151 Vgl. dazu auch Becker/Köster/Sandmann (2006), S. 503. MERTENS/GRIESE haben in diesem Zusammenhang bei einer Stichprobe ermittelt, dass Führungskräfte pro Arbeitstag 23 ausgedruckte Berichte vorgelegt bekommen. Vgl. Mertens/Griese (2002), S. 68.

152 Vgl. Liebetruth/Otto (2006), S. 13. 
Eine weitere Störungsursache, die zur Informationsüberflutung der Berichtsempfänger beiträgt, ist die Granularität der auszuwertenden Informationen. 153 RECHKEMMER zufolge leidet die Informationsversorgung der Unternehmungsführung, die Überblick verschaffende Informationen nachfragen, an einer Überbetonung operativer Aspekte. ${ }^{154}$ Will eine Unternehmung jederzeit flexibel auf Marktveränderungen reagieren, sind die Führungskräfte hingegen auf strategische Informationen angewiesen. ${ }^{155}$ KÜNKELE/SCHÄFFER bemerken darüber hinaus, dass eine $\mathrm{zu}$ detaillierte Informationsversorgung $\mathrm{zu}$ redundanten Daten führt, deren Verarbeitung in unnötiger Weise die Ressourcen der Unternehmung beansprucht. ${ }^{156}$ Den Ausführungen von BLOHM zufolge führt eine übertriebene Genauigkeit der berichteten Informationen sowohl zu unnötigen Kosten als auch zu Verzögerungen. 157 Gleichzeitig verweisen einige Autoren auf die Gefahr irrationalen und aktionistischen Verhaltens betrieblicher Entscheidungsträger als Folge einer Informationsüberlastung aufgrund von zu detaillierter Informationen. ${ }^{158}$

Zur Vermeidung der oben beschriebenen Defizite lassen sich z. B. Informationsbedarfsprofile der Berichtsanwender einsetzen. Derartige Profile, die im Vorfeld der Berichtsproduktion erstellt werden müssen, ermöglichen eine benutzerabhängige Informationsversorgung. Um den Berichtsempfängern zudem eine hohe perzeptive Aufnahmefähigkeit der Berichtsinhalte zu ermöglichen, empfiehlt GÖPFERT die Einhaltung von Gestaltungsregeln, die die Anzahl, den Verdichtungsgrad sowie die Übersichtlichkeit der berichteten Informationen vorgeben. 159 Diese Erkenntnis wird auch von HIRSCH/PAEFGEN/SCHAIER bestätigt, die im Rahmen ihrer Feldstudie feststellen, dass die Ist-Gestaltung von Monatsberichten von der Soll-Gestaltung der Reports abweicht. ${ }^{160}$ Mit Blick auf die Wichtigkeit, Berichte möglichst empfängerindividuell zu gestalten, schlägt BANGE vor, die Stelle eines Report-Designers einzurichten, dessen Auf-

153 Vgl. Becker/Seidel/Janiesch (2007), S. 606; Krause/Schmitz (2006), S. 351. Die Granularität steht für die Stufe des Verdichtungsgrades von Daten. Vgl. Bauer/Günzel (2004), S. 528 .

154 Vgl. Rechkemmer (1998), S. 380.

155 Vgl. Stock/Büttner (2004), S. 1064.

156 Vgl. Künkele/Schäffer (2007), S. 78. Auf die Gefahr einer Doppelberichterstattung verweist auch Blohm (1980), S. 319.

157 Vgl. Blohm (1980), S. 319.

$158 \mathrm{Zu}$ diesen Autoren gehören beispielsweise CHIA und DöRnER. Vgl. Chia (1995), S. 814f.; Dörner (1989), S. $151 \mathrm{ff}$.

159 Vgl. Göpfert (2002), S. 152. In ihrem Beitrag liefert die Autorin weitere Gestaltungsregeln für Berichte. Vgl. Göpfert (2002), S. 152ff.

160 Vgl. Hirsch/Paefgen/Schaier (2008), S. 331. Als Grund für dieses Defizit geben die Autoren eine mangelnde systematische Kommunikation zwischen den Berichtserstellern und -empfängern an. Vgl. Hirsch/Paefgen/Schaier (2008), S. 332. 
gabenbereich die Durchführung notwenig gewordener Anpassungen am Inhalt sowie am Layout der Berichte umfasst. ${ }^{161}$

Für einen effektiven Berichtsprozess mit dem Ziel einer empfängerorientierten Informationsbereitstellung ist darüber hinaus anzustreben, den jeweiligen Berichtsempfängern nur diejenigen Informationen zur Verfügung zu stellen, die tatsächlich eine Relevanz für diesen Empfängerkreis haben. ${ }^{162}$ Die in diesem Zusammenhang vom internen Berichtswesen zu bewältigende Herausforderung liegt darin, den Berichtsempfängern nicht nur ausgewählte monetäre Kennzahlen, sondern alle weiteren, zum Verständnis notwendigen Informationen zu präsentieren, die z. B. unterschiedliche Unternehmungsbereiche betreffen. ${ }^{163} \mathrm{Als}$ weitere Störungsursache ist das Defizit zu erkennen, dass die berichteten Informationen, die aus der Rechnungslegung stammen, vergangenheitsorientiert sind, ${ }^{164}$ während die Unternehmungsführung auf zukunftsorientierte Berichtsinhalte angewiesen ist. 165

Mit Blick auf die mangelnde Relevanz der Informationen aus dem externern Berichtswesen stellen WAGENHOFER und LEV/ZAROWIN fest, dass das Reporting von Finanzinformationen in den vergangenen Jahren an Aussagekraft verloren hat. ${ }^{166}$ In diesem Zusammenhang kritisieren MÜLLER/KLATT/PFITZMEYER, dass das Reporting deutscher Konzerne im hohen Maß durch die Fokussierung auf finanzielle Größen geprägt ist, die sich den klassischen Bestandteilen eines Jahresabschlusses wie z. B. Bilanz, GuV-Rechnung oder Kapitalflussrechnung entnehmen lassen. ${ }^{167}$ Die Autoren stellen darüber hinaus fest, dass nicht finanzielle Größen wie z. B. die Kündigungsrate eine untergeordnete Bedeutung für die Steuerung einer Unternehmung zu haben scheinen. 168

Eine verstärkte Nachfrage nach zusätzlichen Informationen wird sich nach WAGENHOFER auch auf die immateriellen Anlagewerte sowie die Werttreiber einer Unternehmung richten. ${ }^{169}$ Darüber hinaus prognostizieren XIAO/JONES/ LYMER, dass in den kommenden Jahren neben qualitativen Informationen auch Informationen nachgefragt werden, die nicht der Wirtschaftsprüfung unterliegen

Vgl. Bange (2004), S. 94.

Vgl. dazu Horváth (2006), S. 601.

Welche Informationen zur Erfüllung der Berichtszwecke von einem betrieblichen Berichtswesen bereitzustellen sind, lässt sich der Aufzählung von GÖPFERT und GÖRICKE/ KIRCHHOF entnehmen. Vgl. Göpfert (2002), S. 147; Göricke/Kirchhof (2006), S. 55.

Vgl. Scheer/Bungert (2004), S. 13.

Vgl. Rechkemmer (1998), S. 380.

Vgl. Wagenhofer (2003), S. 263; Lev/Zarowin (1999), S. 383.

Vgl. Müller/Klatt/Pfitzmeyer (2000), S. 355; Gleich et al. (2002), S. 337. Zu dieser Erkenntnis kommen im Rahmen ihrer Auswertung auch Hirsch/Paefgen/Schaier (2008), S. 328.

Vgl. Müller/Klatt/Pfitzmeyer (2000), S. 355.

Vgl. Wagenhofer (2003), S. 266. 
und eine Aussage über die zukünftige Entwicklung der berichtenden Unternehmung liefern. ${ }^{170} \mathrm{Um}$ der wachsenden Dynamik sowie Komplexität im Unternehmungsumfeld zu begegnen, sind gemäß der Forderung von GÖPFERT zufolge weitere Anstrengungen notwendig, das Berichtswesen im Hinblick auf eine stärkere Außen- sowie Zukunftsorientierung auszurichten. ${ }^{171}$

Festzuhalten ist, dass die Verarbeitung der Informationsmenge mit Anstrengungen verbunden ist, die wiederum zu Kosten führen. Neben den Lernkosten, die infolge der Nutzung eines IT-Systems entstehen, treten nach WALL Arbeitsleid bzw. Opportunitätskosten auf. Diese Kosten entstehen, da Manager angesichts der zu verarbeitenden Informationsmenge und knapper Ressourcen auf andere Aktivitäten verzichten müssen. 172

\subsubsection{Niedrige Informationsaktualität}

Ausgehend von der Erkenntnis, dass die Aktualität von Informationen einen positiven Einfluss auf die Effektivität der Handlungen betrieblicher Entscheidungsträger ausübt, ${ }^{173}$ ist in der verzögerten Informationsversorgung eine weitere Störungsursache für die Effektivität der Verarbeitung berichteter Informationen zu identifizieren. ${ }^{174}$ Diese Einschätzung wird auch von GÖPFERT gestützt. Nach ihrer Auffassung stellt die rechtzeitige Verfügbarkeit von Informationen einen zentralen Faktor für den Erfolg unternehmerischer Tätigkeit dar. ${ }^{175}$ Werden Informationen nach ihrem Eintreffen mit einer zu großen Verzögerung veröffentlicht, besteht generell die Gefahr, dass Berichtsinhalte hinsichtlich ihrer Aussagekraft an Bedeutung verlieren. ${ }^{176}$ HOFFMANN/KUSTERER bemerken in diesem Zusammenhang, dass die Informationsbereitstellung (time to information) einem wachsenden Zeitdruck ausgesetzt ist. 177 Während die Unternehmungsführung auf aktuelle Informationen angewiesen ist, muss konstatiert werden, dass Berichtsdaten oftmals mit einem beträchtlichen Zeitverzug zur Verfügung stehen. ${ }^{178} \mathrm{Im}$ Extremfall liegen die Informationen, beispielsweise bei der Nutzung eines DWH-Systems, überhaupt nicht vor. 179

170 Vgl. Xiao/Jones/Lymer (2002), S. 255. Zu einer identischen Einschätzung kommt auch HORVÁTH, nach dessen Auffassung Informationen, die nicht aus dem Rechnungswesen stammen, zukünftig an Bedeutung gewinnen werden. Vgl. Horváth (2006), S. 602.

Vgl. Göpfert (2002), S. 156.

172 Vgl. Wall (2008), S. 85.

173 Vgl. Chia (1995), S. $821 \mathrm{ff}$.

174 Vgl. Künkele/Schäffer (2007), S. 78.

175 Vgl. Göpfert (2002), S. 144.

176 Vgl. Posselt (1986), S. 78.

177 Vgl. Hoffmann/Kusterer (1997), S. 49.

178 Vgl. Rechkemmer (1998), S. 380.

179 Vgl. Stock/Düsing (2003), S. 188. 
Werden die Daten eines IS nicht regelmäßig aktualisiert, besteht generell die Gefahr, dass diese veralten und somit für eine weitere Verwendung unbrauchbar sein können. ${ }^{180}$ Ein Beispiel für eine schnell eintretende Alterung von Informationen lässt sich in der nachlassenden Aktualität von Aktienkursen feststellen, die je nach Erkenntnisstand und Informationsbedarf des Informationsempfängers eine nur sehr begrenzte Aussagekraft aufweisen können. Gleichzeitig müssen auch die Informationen, die die Entwicklung eines Aktienkurses beeinflussen, in Form eines continuous reporting zeitnah und in regelmäßigen Abständen bereitgestellt werden. ${ }^{181}$

Die Bereitstellung aktueller Informationen ist nicht nur aus dem Blickwinkel des internen Berichtswesens von Bedeutung. Auch aus der Perspektive des externen Reportings ist die Veröffentlichung von Informationen über die wirtschaftliche Lage einer Unternehmung, beispielsweise in Form eines Jahres- oder Konzernabschlusses, umso nützlicher, je näher sie publiziert werden. ${ }^{182}$ Die Anforderung, Informationen zur wirtschaftlichen Situation einer Unternehmung zeitnah zur Verfügung zu stellen, wird insbesondere von den Akteuren des Kapitalmarkts geäußert. 183 WEBER ET AL. fügen hinzu, dass insbesondere die Bereitstellung unterjähriger Informationen und von Informationen, die nicht im Geschäftsbericht stehen, die Verlässlichkeit und Kontinuität der Unternehmung herausstellen. ${ }^{184} \mathrm{Da}$ den vergangenheitsorientierten Informationen aus dem externen Rechnungswesen der Bezug zu den Geschäftsprozessen fehlt, ist zudem das Problemfeld zu identifizieren, dass den Entscheidungsträgern keine aktuellen Informationen zu den Unternehmungsabläufen zur Verfügung stehen. 185

Ein weiteres Problemfeld, das die Aktualität der berichteten Informationen mindern kann, hängt mit der Zunahme der seit Jahren zu beobachtenden Komplexität des Berichtswesens zusammen. Die Komplexität resultiert aus der Existenz paralleler sowie miteinander konkurrierender Informationsströme zwischen den berichtenden und den berichtsempfangenden Unternehmungseinheiten, die eine zeitnahe IT-gestützte Bereitstellung der Unternehmungszahlen gefährden. ${ }^{186}$ Die Aktualität der Berichte wird dabei in hohem Maß durch die Unternehmungsstruktur beeinflusst. Liegt beispielsweise ein Konzernverbund vor, so sind u. U. Berichte von Hunderten von Tochterunternehmungen, die zudem hin-

180 Vgl. Leser/Naumann (2007), S. 323.

181 Vgl. Wagenhofer (2003), S. 265.

182 Vgl. Schruff/Kayser (2002), S. 349. Die qualitativ hochwertige und fristgerechte Bereitstellung dieser Informationen wird daher oftmals als kritisch betrachtet. Vgl. Hermann/ Müller (2008), S. 236.

Vgl. Göricke/Kirchhof (2006), S. 54.

184 Vgl. Weber et al. (2002), S. 45.

185 Vgl. Scheer/Bungert (2004), S. 13.

186 Vgl. Müller/Klatt/Pfitzmeyer (2000), S. 355. Zu den daraus resultierenden Problemfeldern vgl. Göricke/Kirchhof (2006), S. 55. 
sichtlich ihrer Branche, Region und Rechtsform unterschiedlich ausgeprägt sein können, ${ }^{187}$ in den entsprechenden IS zusammenzuführen und für ein zeitnahes internes sowie externes Reporting aufzubereiten. ${ }^{188}$ Potenziert wird dieses Problem, wenn sich die Konzernstrukturen häufig ändern, so dass sich ein inhaltlicher und technischer Reportingstandard nicht etablieren kann. ${ }^{189}$ Als komplexitätssteigernd wirkt sich darüber hinaus die Verpflichtung für Unternehmungen aus, unterschiedliche Rechnungslegungsvorschriften zur externen Darstellung parallel anzuwenden, 190 um auf dem Kapitalmarkt zu operieren oder weil das Wirtschaftsrecht am Standort der berichtenden Unternehmung dies erfordert. 191

\subsubsection{Inhaltsbezogene Defizite für die Prozesse „Berichtserstellung“ und „Berichtsaufnahme“}

Die bisher diskutierten Problemfelder erweisen sich insbesondere dann als kritisch, wenn während der Verarbeitung der berichteten Informationen nicht klar ist, um welche Inhalte es sich handelt bzw. welche Bedeutung diese haben. Derartige semantisch bedingte Störungen, die die inhaltliche Dimension eines Berichts betreffen, entstehen, wenn Berichtssender und -empfänger den berichteten Informationen eine unterschiedliche Bedeutung beimessen. ${ }^{192}$ Diese Problematik ist zu erwarten, wenn Informationen aus verschiedenen Quellen zusammengeführt werden müssen, die infolge ihres dezentralen Ursprungs unterschiedlich definiert sind. ${ }^{193}$ Betroffen von diesem Defizit sind dabei insbesondere die Kernprozesse Berichtserstellung und -aufnahme.

BECKER/KÖSTER/SANDMANN bemerken dazu, dass sich hinter den zur Unternehmungssteuerung verwendeten Kennzahlen oftmals ein abweichendes individuelles Verständnis mit unterschiedlichem Informationsgehalt verbirgt. ${ }^{194}$ Problematisch ist z. B. die Verwendung unterschiedlicher Währungen und Metriken, nicht-normierter Kennzahlen oder Bewertungsmethoden. ${ }^{195}$ Liegen beispielsweise einer Bank Jahresabschlüsse kreditsuchender Unternehmungen in unter-

Vgl. Mertens (1999), S. 412.

Siehe dazu auch die Feststellung von Gleich et al. (2002), S. 337.

Vgl. Nutz/Strauß (2002), S. 448.

$\mathrm{Zu}$ diesen gehören beispielsweise für deutsche Unternehmungen neben den Rechnungslegungsvorschriften nach HGB auch die Vorschriften nach IFRS und zum Teil USGAAP. Vgl. dazu Göricke/Kirchhof (2006), S. 54.

Vgl. Mertens (1999), S. 412.

Vgl. dazu Kuhn (1990), S.196

Ein Indiz, dass Informationen aus verschiedenen Quellen „zusammengesetzt“ werden müssen, ist nach der Auffassung von BLOOM der Einsatz des Tabellenkalkulationsprogramms Microsoft ExCEL. Vgl. Bloom (2008), S. 10.

Vgl. Becker/Köster/Sandmann (2006), S. 502.

Vgl. Mertens (1999), S. 412. 
schiedlichen Rechnungslegungsstandards vor, wird die schnelle Analyse der Geschäfts- und Finanzinformationen durch das Ausbleiben zusätzlicher, die einzelnen Werte erklärender Informationen erschwert. 196

Mit Blick auf die Untersuchung von HIRSCH/PAEFGEN/SCHAIER ist in diesem Zusammenhang festzuhalten, dass die fehlende Kommentierung von Informationen - beispielsweise infolge eines Zeitdrucks oder mangelnder Kenntnisse ein wichtiges Problemfeld für die Berichtsempfänger darstellt. ${ }^{197}$ Existiert keine Übereinkunft hinsichtlich der Bedeutung der zu analysierenden Informationen, sind einer automatisierbaren Verarbeitung der Jahresabschlussinformationen mit dem Ziel einer schnellen Vergleichbarkeit wichtiger Kennzahlen enge Grenzen gesetzt. ${ }^{198}$ Um eine Vergleichbarkeit der Jahresabschlüsse von Unternehmungen zu realisieren, die nach unterschiedlichen Rechnungslegungsstandards bilanzieren, bleibt als Ausweg oftmals nur die Anwendung aufwendiger und manueller Transformationsschritte. 199

Verstärkt wird das Defizit einer ineffektiven semantischen Verarbeitung der Berichtsinhalte, wenn für die Berichtsempfänger nicht erkennbar ist, welche Ausgangsdaten verarbeitet und welche Transformationsschritte zur Aufbereitung der Inhalte angewendet worden sind. ${ }^{200}$ Werden Berichte dezentral abgelegt, ist oft unklar, welcher Mitarbeiter an welchen Daten zu welchem Zeitpunkt Änderungen vorgenommen hat. ${ }^{201}$ Diese Defizite führen zur Gefahr, dass die Informationen, die zwischen unterschiedlichen IS ausgetauscht und anschlieBend zur Berichtserstellung aufbereitet werden, den in ihnen enthaltenen $\mathrm{Zu}$ sammenhang verlieren können. ${ }^{202}$ Ein solcher wird vermittelt, wenn ein Bericht weitere Angaben zu den berichteten Inhalten, zur verwendeten Währung oder zum zeitlichen Bezug einer Wertausprägung enthält. ${ }^{203}$ Ist eine eindeutige inhaltliche (Re-)Interpretation der ausgetauschten Informationen nicht oder nur unter Inkaufnahme hoher Aufwendungen möglich, lassen sich insbesondere die

Vgl. Kranich/Schmitz (2003), S. 77.

Vgl. Hirsch/Paefgen/Schaier (2008), S. 331.

Welche unterschiedlichen Auslegungen bei der Interpretation einer Kennzahl auftreten können, wird am Beispiel der Kennzahl „Umsatz“ deutlich. Siehe dazu das Beispiel bei Becker/Köster/Sandmann (2006), S. 503. Die Problematik, die sich aus einer unterschiedlichen Auslegung der verwendeten Begriffe eines Berichts ergeben kann, wird auch im Beitrag von BLOHM deutlich. Vgl. Blohm (1980), S. 319. Vgl. Nutz/Strauß (2002), S. 448. Vgl. dazu auch Abschnitt 2.3.3.2.

Vgl. Becker/Köster/Sandmann (2006), S. 502. Auf das Problem, die Quellen der in einem Bericht präsentierten Daten zu identifizieren, verweisen auch Willis/Hannon (2005), S. 58.

201 Vgl. Eckerson/Sherman (2008), S. 24.

202 Vgl. dazu Meall (2007), S. 73. Auf die Bedeutung, Zusammenhänge zwischen den berichteten Daten abzubilden, verweist Besemer (2008), S. 12.

Vgl. Willis/Hannon (2005), S. 58. 
Potenziale des World Wide Web (WWW) zur schnellen Verarbeitung der berichteten Informationen nicht vollständig nutzen. ${ }^{204}$

Das oben beschriebene Problem tritt insbesondere dann auf, wenn Berichte aus unterschiedlichen Quellen stammen. Obwohl DWH-Systeme die Möglichkeit bieten, Berichte aus einem konsistenten und vereinheitlichten Informationsbestand zu erstellen, ist das betriebliche Berichtswesen i. d. R. dadurch gekennzeichnet, dass Informationen aus unterschiedlichen Quellen den Führungskräften getrennt voneinander präsentiert werden. ${ }^{205}$ In diesem Fall ist es die Aufgabe der Geschäftsleitung, aus den verschiedenen Informationsquellen ein Gesamtbild zu erstellen, was zur Folge haben kann, dass erneut zweckbezogene Defizite auftreten können. ${ }^{206}$

Den Vorschlag von КосH aufgreifend, lassen sich die semantischen Störungsursachen in derivative und originäre Störungen unterscheiden. ${ }^{207}$ Während derivate Störungen Folge einer syntaktischen Störung sind, treten originäre Störungen unabhängig von einer technisch bedingten, fehlerhaften Verarbeitung der Informationen auf. Während in dem vorliegenden Abschnitt originäre semantische Störungsursachen thematisiert wurden, fokussiert der folgende Abschnitt Defizite, die zu derivativen semantischen Störungen führen.

\subsubsection{Syntaxbezogene Defizite für alle Teilprozesse des Berichtswesens}

Trotz der Bedeutung des Berichtswesens als wichtiges Instrument für eine erfolgreiche Unternehmungssteuerung ist $\mathrm{zu}$ bemerken, dass eine effiziente Verarbeitung von Berichtsinformationen nur in Einzelfällen von den Unternehmungen betrieben wird. ${ }^{208}$ Mit Blick auf diesen Aspekt ist zu konstatieren, dass die Verarbeitung der berichteten Informationen die Gefahr einer zeitaufwendigen, kostenintensiven und fehleranfälligen Tätigkeit birgt. ${ }^{209}$ Derartige syntaxbezogene Ineffizienzen ergeben sich sowohl im Rahmen des internen als auch externen Berichtswesens, ${ }^{210}$ wobei alle Kernprozesse des Berichtswesens von diesen Defiziten betroffen sind.

Wie in den folgenden Ausführungen deutlich wird, lassen sich zwei syntaxbezogene Störungsursachen identifizieren, die eine effiziente Ausführung der Kernprozesse im Reporting beeinträchtigen. Ausgehend von technisch bedingten Bedrohungspotenzialen, die in Abschnitt 2.3.3.1 thematisiert werden, fokus-

Vgl. Meyer-Pries/Gröner (2002), S. 44.

Vgl. Mertens (1999), S. 406.

Vgl. Abschnitt 2.3.1.

Vgl. Koch (1994), S. 73.

Vgl. Becker/Seidel/Janiesch (2007), S. 605; Becker/Seidel/Janiesch (2008), S. 229.

Vgl. Brown/Willis (2003), S. 70.

Nutz/Strauß (2002), S. 448. 
siert Abschnitt 2.3.3.2 Defizite, die sich infolge personenbedingter Störungen ergeben.

\subsubsection{Technisch bedingte Störungen}

Wie andere Anwendungssysteme, die zur Unterstützung eines bestimmten betrieblichen Aufgabenbereichs zum Einsatz kommen, unterliegen auch die Applikationen, die für die Zwecke des Reportings bestimmt sind, der Gefahr, dass die ihnen vorgehaltenen Daten fehlerhaft oder unzuverlässig sein können. ${ }^{211}$ Ein Grund für derartige Daten lässt sich in einer syntaktisch fehlerhaften Übertragung und Verknüpfung der berichteten Informationen durch die zum Einsatz kommenden IS identifizieren. ${ }^{212}$

Aus dem Blickwinkel der IT betrachtet, wird eine effiziente Verknüpfung der Informationsquellen aufgrund von unterschiedlichen Betriebssystemplattformen und Datenbanksystemen erschwert. ${ }^{213}$ Zwar lassen sich für die Zwecke der Datenintegration DWH-Systeme nutzen. Jedoch wird die Herausforderung, heterogene IS zu integrieren, angesichts der Vielzahl der betriebenen DWHAnwendungen auf eine höhere Ebene verlagert.214 Die Heterogenität der zum Einsatz kommenden Softwaresysteme äußert sich darin, dass sowohl verschiedene Ausprägungen von Datenmodellen bzw. -strukturen als auch diverse Zugriffs-, Präsentations- und Austauschformate zur Verarbeitung der Informationen genutzt werden. ${ }^{215}$ Abbildung 9 liefert eine Zusammenstellung gängiger Datenstrukturen, Zugriffs-, Präsentations- und Austauschformate, die in den meisten Berichtsanwendungen zum Einsatz kommen.

Mit Blick auf die Datenstrukturen ist festzustellen, dass in den vergangenen Jahrzehnten eine Reihe von Ausprägungen von kommerziellen und nichtkommerziellen Datenbanksystemen entstanden ist, die verschiedene Konzepte zur Datenmodellierung unterstützen. Neben den am meisten verbreiteten relationalen Datenbanksystemen existieren weitere Varianten von Datenbanksystemen, die objektorientierte, objekt-relationale oder multidimensionale Konzepte

211 Vgl. dazu Becker/Köster/Sandmann (2006), S. 503.

212 Vgl. Kuhn (1990), S. 196.

213 Vgl. Mertens (1999), S. 412. Auf die Vielzahl der zum Einsatz kommenden datenhaltenden Systeme zur Verarbeitung von Geschäftsinformationen verweist auch Pandrangi (2003).

214 Auf die Problematik, dass in den Unternehmungen viele DWH-Systeme und unabhängige Data Marts betrieben werden - beispielsweise infolge von Unternehmungszusammenschlüssen - verweist Bloom (2008), S. 10.

215 MEYER-PRIES/GRÖNER bemerken in diesem Zusammenhang, dass beispielsweise der Austausch von Abschlussdaten mit dem Problem konfrontiert ist, dass die am Austausch beteiligten Partner voneinander abweichende Datensatzstrukturen verwenden können. Vgl. Meyer-Pries/Gröner (2002), S. 51. 
unterstützen. Darüber hinaus werden heute immer noch netzwerkorientierte und hierarchische Datenbanksysteme betrieben, die in der Anfangszeit moderner Datenverarbeitung entstanden sind. 216

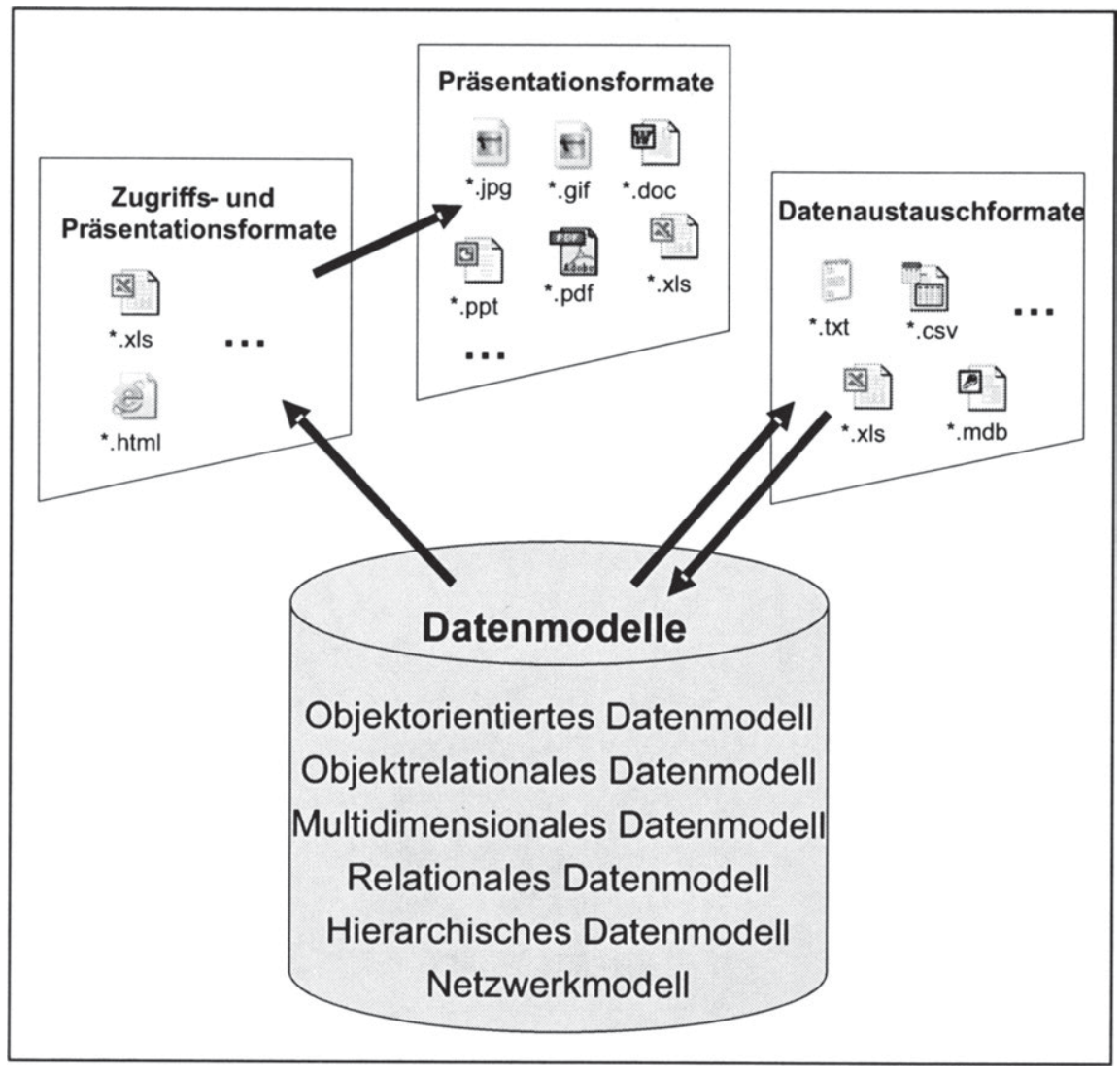

Abbildung 9: Heterogenität der Datenstrukturen und Zugriffs-, Präsentations- sowie Datenaustauschformate

Neben der Vielfalt der datenhaltenden Systeme und der von diesen unterstützten Konzepte zur Datenverarbeitung ist im gleichen Maße eine Heterogenität an Zugriffs-, Präsentations- und Datenaustauschformaten festzustellen, die genutzt

$216 \mathrm{Zu}$ den Varianten eines Netzwerkmodells, hierarchischen, relationalen und objektorientierten Datenmodells vgl. Rob/Coronel (2007), S. 32ff.; Gabriel/Röhrs (1995), S. $114 \mathrm{ff}$;; Behme/Ohlendorf (1994), S. 126ff. 
werden, um aus einem zugrunde liegenden Datenbestand Berichte zu generieren und sie einem weiteren Nutzerkreis zu präsentieren. ${ }^{217}$

Um auf die Daten eines DBS oder DWH-Systems, beispielsweise im Zuge der Berichtserstellung, zuzugreifen, werden nicht selten gängige Datenformate präferiert, zu denen beispielsweise die HYPERTEXT MARKUP LANGUAGE (HTML) oder MICROSOFT EXCEL SPREADSHEET-Dateien (xls) gehören. ${ }^{218}$ Gerade Tabellenkalkulationsprogramme wie MICROSOFT EXCEL stellen die einfachsten Sammel- und Auswertungswerkzeuge dar,219 die beispielsweise über eine ODBC-Schnittstelle an ein DBS angebunden werden können. ${ }^{220}$ HTML wird in den gängigen Berichtsanwendungen verwendet, um ein Web-Reporting zu ermöglichen. Dies lässt sich bewerkstelligen, indem Berichte mit Hilfe eines BITools erstellt, in einem proprietären Format gespeichert und anschließend in ein HTML-Dokument transformiert werden. Nachdem der als HTML-Dokument vorliegende Report auf einem Web-Server abgelegt wird, lässt sich der Bericht über eine URL in einem Web-Browser aufrufen. Dabei werden die Daten aus dem angebundenen Quell- bzw. Backendsystem geladen.221

Bei den Präsentationsformaten, die ausschließlich zur Darstellung eines Berichts genutzt werden können, lassen sich neben HTML beispielsweise das PORTABLE DOCUMENT FORMAT (PDF) sowie sämtliche Office-Formate nutzen.222 Um einen Datenaustausch zwischen einem Quellsystem und einer ZielAnwendung $\mathrm{zu}$ ermöglichen, lassen sich beispielsweise Datenformate wie “.txt", ".csv", ".mdb" oder ".xls" einsetzen.223 Neben den in der Abbildung präsentierten Formaten existieren für die verschiedenen Anwendungsbereiche dar-

217 Vgl. dazu auch Hahn (2003), S. 606.

218 Die Dateiendung "xls" steht für ExCEL SPREADSHEET. Bei einem ExCEL SPREADSHEET handelt es sich um eine Arbeitsmappe, die mit dem Tabellenkalkulationsprogramm MiCROSOFT EXCEL erstellt wurde. Die Funktionalität, über einen gängigen Web-Browser oder über MiCROSOFT ExCEL auf einen Datenbestand zuzugreifen, wird heute nahezu von allen Datenbanksystemen bereit gestellt.

219 Vgl. Hahn (2003), S. 605. SCHMITZ zufolge haben im Jahr 2008 65\% der mittelständischen Unternehmungen ExCEL zur Dateneingabe genutzt. Vgl. Schmitz (2008), S. 12. BANGE/FRIEDRICH bemerken, dass nur $17 \%$ der im Rahmen ihrer Studie Befragten bei der Berichtserstellung und Analyse auf ExCEL verzichten. Vgl. Bange/Friedrich (2008), S. 35 .

220 Zu den Vorteilen, die ExCEL für die Zwecke des Reportings bietet, und den Möglichkeiten zur ExCEL-Anbindung von BI-Lösungen und OLAP-Datenbanken vgl. Talarczyk (2008), S. 36ff.

221 Zur WWW-Integration von Datenbanken vgl. z. B. Kleinschmidt/Rank (2002), S. $151 \mathrm{ff}$.

222 Vgl. dazu Schruff/Kayser (2002), S. 353; Gluchowski/Pastwa (2006), S. 66; Besemer (2008), S. 10.

223 Während die Dateiendung ".txt" eine einfache Textdatei markiert, stehen die Dateierweiterungen ".csv" und ".mdb" für Comma oder Character Separated Values bzw. für das Dateiformat MICROSOFT DATABASE. 
über hinaus weitere proprietäre bzw. herstellerspezifische Formate, die für den Datenzugriff, die -präsentation und den -austausch verwendet werden.224

Als Folge dieser Heterogenität lässt sich das Problem ableiten, dass bei der Übermittlung von Daten Ineffizienzen auftreten können.225 Dieses Defizit wird verstärkt, wenn unternehmungsexterne Informationen verarbeitet werden müssen, da sie hinsichtlich ihrer Quelle und Art tendenziell inhomogener sind als unternehmungsinterne Informationsquellen. ${ }^{226}$ Liegen unterschiedliche Formate oder Datenstrukturen vor, sind diverse Transformationsverfahren einzusetzen, mit deren Hilfe die Datenobjekte samt ihrer Beziehungen und Wertausprägungen eines IS in die Datenstruktur eines anderen datenhaltenden Systems überführt werden können, das andere Konzepte zur Datenmodellierung und zur Datenspeicherung unterstützt. Die hier beschriebene Problematik wird verstärkt, wenn kein Datenbanksystem eingesetzt und die Daten in hundert- bis tausendfach verstreuten Dateien verwaltet werden.227

Eine wichtige Voraussetzung, damit sowohl die Transformationsverfahren zum Mapping der Datenfelder unterschiedlicher Datenstrukturen angewendet als auch die Format- bzw. Medienbrüche beseitigt oder reduziert werden können, liegt in der Bereitstellung entsprechender Schnittstellen. Sind diese nicht gegeben, müssen neue Schnittstellen erstellt oder bestehende modifiziert werden, damit eine automatisierte Datentransformation zwischen den am Austauschprozess beteiligten IS erfolgen kann. I. d. R. erweist sich allerdings die Erstellung sowie die anschließende Wartung der Systemschnittstellen als eine sehr kostenintensive Tätigkeit. ${ }^{228}$

\subsubsection{Personen bedingte Störungen}

Die Existenz von Format- und Medienbrüchen führt zu einem weiteren Problem, wenn eine automatisierte Datenübernahme selbst unter hohen Aufwendungen nicht möglich ist. Die Problematik, die aus der Nutzung vielfältiger, inkompatibler Dateiformate resultieren kann, ist insbesondere dann kritisch, wenn neben der Verwendung digitaler Dokumente und Dateien zusätzlich physische

224 Die dreifachen Punkte in der Abbildung 9 dienen dabei als Platzhalter, um die Vielfalt der proprietären Formate anzudeuten, die in dieser Arbeit nicht mehr erwähnt werden.

225 Vgl. Nutz/Strauß (2002), S. 448.

226 Vgl. Mertens (1999), S. 413. MERTENS zufolge liegt in der mangelnden Anbindung von externen qualitativen Informationen die Ursache für die geringe Akzeptanz von Management-Informationssystemen. Vgl. Mertens (1999), S. 414.

227 Vgl. Talarczyk (2008), S. 36.

228 Vgl. Meyer-Pries/Gröner (2002), S. 51. 
Medien genutzt werden.229 Ein solches Problem ist z. B. gegeben, wenn die auszutauschenden Informationen in ausgedruckten Berichtsdokumenten enthalten sind und anschließend manuell in ein IS zur Speicherung und/oder weiteren Bearbeitung übertragen werden müssen. ${ }^{230}$ In diesem Zusammenhang ist $\mathrm{zu}$ konstatieren, dass fehlerhafte oder unzuverlässige Daten oftmals infolge einer manuellen Dateneingabe auftreten. 231

Tippfehler führen beispielsweise dazu, dass die in ein Datenbanksystem eingepflegten Werte entweder falsch eingegeben sind oder bereits vorhanden sein können. Problematisch ist darüber hinaus, wenn die Daten über verschiedene Kanäle in ein oder gar mehrere IS gelangen. Neben den digitalen Kommunikationswegen wie beispielsweise Web-Formulare oder E-Mail führen nach wie vor klassische Kommunikationsmedien, zu denen z. B. das Telefon oder der Briefverkehr gehören, zur Aufnahme und Übertragung fehlerhafter oder unzuverlässiger Daten in die zur Datenhaltung vorgesehenen IS. Neben den technisch bedingten Problemfeldern lassen sich daher Personen bedingte Störungsursachen identifizieren, die die Effizienz der Kernprozesse im Reporting gefährden.

Neben den in Abschnitt 2.3.3.1 bereits identifizierten Defiziten als Folge einer technisch bedingten fehlerhaften und unzuverlässigen Datentransformation verweisen SAMTLEBEN/STADLBAUER/HESS auf weitere, die aus einer manuellen Dateneingabe resultieren können. Im Fokus stehen dabei mögliche Kosten- und Zeitnachteile. 232 Kostennachteile ergeben sich dadurch, dass für die manuelle Datenübernahme Personal beauftragt werden muss. Diese Erkenntnis wird auch von MülleR/KLATT/PFITZMEYER gestützt, die in ihrem Beitrag bemerken, dass insbesondere der geringe Automatisierungsgrad bei der Verarbeitung von Berichtsinformationen ein weiterer Hinderungsgrund für eine effiziente Berichterstattung ist, da viele Ressourcen für die manuellen Abstimmungs- und Korrekturarbeiten an den Informationen aufgewendet werden müssen, die nicht zur Wertschöpfung beitragen.233 Mit Blick auf den Zeitaspekt ist zu konstatieren, dass der manuelle, zeitintensive Import der Daten dazu führen kann, dass der

229 Nach WILLIS sind insbesondere Banken mit dem Problem konfrontiert, in einem manuellen Prozess Informationen aus Papierdokumenten, die wiederum aus verschiedenen Quellen stammen, zusammenzuführen. Vgl. Willis (2003), S. 58.

230 Vgl. Göricke/Kirchhof (2006), S. 56.

231 Vgl. Eckerson/Sherman (2008), S. 24; Leser/Naumann (2007), S. 322; Samtleben/ Stadlbauer/Hess (2006), S. 88.

232 Vgl. Samtleben/Stadlbauer/Hess (2006), S. 87.

233 Vgl. Müller/Klatt/Pfitzmeyer (2000), S. 356; Hannon (2006), S. 60. In diesem Zusammenhang ist zu bemerken, dass Mitarbeiter bis zu zwei Drittel ihrer Arbeitszeit für die Kontrolle, Korrektur und Aufbereitung der Daten und Informationen aufwenden müssen. Vgl. Köthner (2006), S. 6. 
Anspruch der Berichtsempfänger hinsichtlich einer zeitnahen Informationsversorgung nicht erfüllt wird. ${ }^{234}$

Ferner muss eine manuelle Mehrfacherfassung der Daten häufig erfolgen, falls es nicht gelingt, mit aufwendigen Transformations- oder Konvertierungsverfahren eine automatisierte Datenübernahme zu erreichen.235 Wie MEYERPRIES/GRÖNER bemerken, ist die Mehrfacherfassung von Daten der internen und externen Berichterstattung insbesondere ein Problem für Konzerne, Verbände, Analysten und Finanzinstitute. ${ }^{236}$ Obwohl viele der kommerziell angebotenen Softwaresysteme und -werkzeuge, die für Reporting-Zwecke eingesetzt werden, gängige Datenformate unterstützen, kann es ferner erforderlich sein, die Daten in ein anderes Format zu transformieren oder zumindest manuell anzupassen, damit eine anschließende Auswertung der Inhalte erfolgen kann. Wegen der Vielfalt der Datenstrukturen und Datenformate ist die manuelle Manipulation der Daten ebenfalls mit der Gefahr verbunden, dass die Daten verloren gehen oder falsch aufbereitet werden.237

Dass Datenfehler sowie redundante Datensätze in den Berichtssystemen auftreten, hängt nicht nur mit menschlicher Nachlässigkeit zusammen. In gleichem Maße kann auch die automatisierte, maschinen- sowie computergestützte Erfassung von Daten beispielsweise durch den Einsatz von Scannern, Importroutinen und/oder Texterkennungsprogrammen zu einer reduzierten Datenqualität führen. ${ }^{238}$ LESER/NAUMANN weisen darauf hin, dass im Allgemeinen fehlerbehaftete Messungen einerseits mit dem Ziel der Kostenersparnis bewusst in Kauf genommen werden, andererseits sich vor dem Hintergrund technischer Restriktionen nicht verhindern lassen. ${ }^{239}$

Ein weiteres Problemfeld, das zu personenbedingten Störungen beitragen kann, ist die Nutzung von Tabellenkalkulationsprogrammen wie MiCROSOFT EXCEL. ${ }^{240}$ Probleme entstehen, wenn in den Arbeitsblättern Makros verwendet werden, die von einem Mitarbeiter entwickelt wurden, der die Unternehmung verlassen hat. ${ }^{241}$ Werden die entsprechenden Arbeitsblätter zwischen den Mit-

Vgl. Samtleben/Stadlbauer/Hess (2006), S. 87f.

Vgl. Nutz/Strauß (2002), S. 448; Göricke/Kirchhof (2006), S. 55.

Vgl. Meyer-Pries/Gröner (2002), S. 45.

Vgl. Becker/Köster/Sandmann (2006), S. 502.

Texterkennungsprogramme werden auch als Optical Character Recognition-Programme (OCR-Programme) bezeichnet. OCR-Programme bieten die Möglichkeit, Texte einzuscannen und anschließend mit Hilfe von Textverarbeitungsprogrammen weiterzuverarbeiten. Vgl. Hansen/Neumann (2005), S. 364.

Vgl. Leser/Naumann (2007), S. 322.

Eine Übersicht über Problemfelder von Microsoft ExcEl liefert Grabowski (2008), S. 23.

Vgl. Eckerson/Sherman (2008), S. 24. 
arbeitern ausgetauscht und weiterverwendet, ${ }^{242}$ ist oft Unkenntnis hinsichtlich der Funktionalität sowie Erweiterungsmöglichkeiten der Makros zu beobachten. ${ }^{243}$ Letztlich führt dieses nicht vorhandene Wissen zu weiteren Kosten- und Zeitnachteilen.

\subsection{Zusammenfassung und kritische Würdigung}

Das vorliegende Kapitel rückte die Prozessunterstützung im betrieblichen Berichtswesen in den Vordergrund. Zur inhaltlichen Fundierung fokussierte Abschnitt 2.1 zunächst die begrifflichen und konzeptionellen Grundlagen des Reportings sowie die Zwecke, die mit dem Berichtswesen in Verbindung stehen. Abschnitt 2.2 präsentierte mit den Berichtssystemen eine Klasse von IS zur Unterstützung der Kernprozesse im Berichtswesen. Aus den Ausführungen des Abschnitts 2.3 wurde deutlich, dass sich eine Reihe von Defiziten identifizieren lässt, die die Effektivität sowie die Effizienz der Kernprozesse beeinträchtigen.

Als zusammenfassendes Ergebnis der Ausführungen dieses Kapitels wird das Berichtswesen einer Unternehmung als Gesamtheit aller Personen, Prozesse und IS aufgefasst, die zur Erzeugung, Aufbereitung, Übermittlung und Auswertung von formalisierten Berichten beitragen. Aus dieser Zieldefinition wird deutlich, dass das dieser Arbeit zugrunde liegende Verständnis des Berichtswesens über die Vorstellung von BECKER/SEIDEL/JANIESCH hinausgeht, da für die Autoren ausschließlich die innerbetrieblichen Entscheidungsträger als Rezipienten der Berichte in Frage kommen. ${ }^{244}$ Um die Informationsverarbeitung zu verbessern, erfolgt in den Berichten i. d. R. eine Visualisierung der vermittelten Inhalte. ${ }^{245}$ Zur informationstechnischen Unterstützung des Berichtswesens kommen Berichtssysteme zum Einsatz.

Vor dem Hintergrund der in Abbildung 2 präsentierten Einordnung des $\mathrm{Be}-$ richtswesens nach GöPFERT ist zu konstatieren, dass der dieser Arbeit zugrunde liegende Definitionsvorschlag der weiten Begriffsauslegung folgt. Kritisch an der engen Auslegung nach BLOHM ist zu werten, dass das Berichtswesen lediglich als Instrument zur Informationsübermittlung verstanden wird. ${ }^{246}$ Auch die von HORVÁTH erweiterte Sichtweise, bei der das Berichtswesen mit der Informationsübermittlung und -produktion gleichgesetzt wird, ist als eine zu enge Perspektive zu beurteilen. ${ }^{247}$

Nach KÖTHNER verschicken 82 Prozent von 500 befragten Unternehmungen EXCELDateien per Mail, um Planungsprozesse durchzuführen. Vgl. Köthner (2008), S. 26.

Vgl. Eckerson/Sherman (2008), S. 24.

244 Vgl. Becker/Seidel/Janiesch (2007), S. 607.

245 Vgl. Kemper/Mehanna/Unger (2004), S. 110.

Siehe dazu die Ausführungen in Abschnitt 2.1.1.

Vgl. Abschnitt 2.1.1. 
Darüber hinaus wird in dieser Arbeit in Abgrenzung zu HoRváTH die Auffassung vertreten, dass das Berichtswesen nicht nur auf das Top-Management sondern ebenfalls auf Berichtsempfänger auszurichten ist, die sich auf allen Hierarchiestufen einer Unternehmung befinden. ${ }^{248}$ Die Ausweitung des Empfängerkreises impliziert, dass in einem Report nicht nur Geschäfts- und Finanzinformationen präsentiert werden, die in aggregierter bzw. konsolidierter Form vorliegen - z. B. infolge einer Abfrage auf das DWH, das einem Berichtssystem zugrunde liegt. ${ }^{249}$ In analoger Weise lassen sich auch disaggregierte Informationen bzw. Detaildaten, die in transaktionsorientierten Systemen verarbeitet werden, in einem Bericht aufnehmen.250 Als Ausprägung einer derartigen Information lässt sich das Beispiel eines Buchungssatzes anführen.

Vor dem Hintergrund der in Abschnitt 2.1.3 thematisierten Berichtszwecke ist ferner zu konstatieren, dass der von HORVÁTH propagierte alleinige Berichtszweck, die ergebniszielorientierte Planung und Kontrolle zu unterstützen, eine zu enge Sichtweise darstellt und daher ebenfalls in dieser Arbeit abgelehnt wird. ${ }^{251}$ In gleicher Weise, wie die Planung und Kontrolle von Bedeutung sind, fokussiert das betriebliche Berichtswesen ebenfalls die Dokumentation von Ereignissen und die Auslösung von Aktivitäten.

Die enge Auslegung des Berichtswesens sowie die Fokussierung auf das Management als ausschließliche Berichtsempfängergruppe haben dazu geführt, dass die enge Interpretation des Reportings von einer Mehrheit der Autoren kritisiert wurde und auch in dieser Arbeit abgelehnt wird.252 Stattdessen wird in dieser Arbeit die Auffassung vertreten, dass die beim Berichtswesen anfallenden Informationsflüsse die Gestaltung aller Kernprozesse im Berichtswesen erfordern, um dem Informationsbedarf der Berichtsempfänger zu genügen. Hierzu ließen sich mit der Informationsbeschaffung, Berichtserstellung, Berichtsdistribution und Berichtsaufnahme vier Kernprozesse identifizieren.

Mit Blick auf die in Abschnitt 2.3 präsentierten Defizite bei der Ausführung der Berichtsprozesse ist aus der Perspektive der Wirtschaftsinformatik die Frage von Interesse, welche aktuell diskutierten Konzepte und Technologien dazu beitragen, die Kernprozesse im betrieblichen Berichtswesen effektiver und effizienter zu gestalten und zu betreiben. Ein potenzieller, in der Literatur aktuell diskutierter Ansatz liegt beispielsweise mit dem Konzept einer Serviceorientierten Architektur (SOA) vor.

Vgl. Bange (2004), S. 94; Leßweng (2003), S. 66.

249 Derartige Informationen werden i. d. R. von Entscheidungsträgern der mittleren und höheren Managementebene nachgefragt.

250 Neben einem hohen Detaillierungsgrad zeichnen sich derartige Daten durch eine hohe Aktualität und Genauigkeit aus. Vgl. Hansen/Neumann (2005), S. 528.

251 Vgl. dazu auch Koch (1994), S. 60.

252 Vgl. Göpfert (2002), S. 144. 
Alexander Pastwa - 978-3-631-75488-7

Downloaded from PubFactory at 01/11/2019 04:20:29AM

via free access 


\section{Serviceorientierte Architekturen (SOA) als innovatives Konzept für eine integrierte Informationsverarbeitung}

Dass die effiziente und effektive Verarbeitung von Informationen durch den Einsatz geeigneter IS einen wichtigen Erfolgsfaktor für eine Unternehmung darstellt, ist anerkanntes Gedankengut in der Betriebswirtschaftslehre und Wirtschaftsinformatik. 253 Dieser Zusammenhang lässt sich insbesondere verdeutlichen, wenn man die Unternehmung als informationsverarbeitendes System modelliert. ${ }^{254}$ Sowohl einzelne betriebliche Funktionen als auch die Durchführung gesamter unternehmungsinterner sowie -übergreifender Geschäftsprozesse hängen von den Informationen sowie den IS ab, die eine teil- oder vollautomatisierte Informationsverarbeitung ermöglichen sollen. Die zur Verfügung stehende Informationstechnik (IT) wird dabei häufig mit dem Ziel eingesetzt, die Wirtschaftlichkeit und Produktivität einer Unternehmung zu verbessern. 255

Als Teil eines IS sind den Anwendungssystemen, die zur Unterstützung der Informationsverarbeitung innerhalb einer geeigneten Anwendungsarchitektur betrieben werden, sowie der Anwendungsarchitektur selbst eine besondere Beachtung zu schenken, da die Entscheidung für eine bestimmte Applikationsarchitektur maßgeblich die Aufwendungen für die Entwicklung, die Einführung, den Betrieb sowie die Wartung der Anwendungssysteme beeinflusst. $256 \mathrm{Da}$ die Geschäftsprozesse von der IT sowie den IT-Anwendungen unterstützt bzw. erst ermöglicht werden, 257 ist in diesem Zusammenhang zu beachten, dass Änderungen an den Geschäftsprozessen zu Modifikationen an der gesamten ISArchitektur einer Unternehmung führen müssen, ${ }^{258}$ die sich wiederum in entsprechenden Aufwendungen niederschlagen. ${ }^{259}$ Die Herausforderung, integrier-

Vgl. Gabriel/Beier (2003), S. 22; Gluchowski/Gabriel/Dittmar (2007), S. 27f.; Hansen/ Neumann (2005), S. 16ff.; Laudon/Laudon/Schoder (2006), S. 27ff.; Horváth (2006), S. $664 \mathrm{ff}$.

Vgl. Gabriel/Röhrs (1995), S. 23ff.

255 Vgl. Holtschke/Heier/Hummel (2009), S. 1.

256 Vgl. Müller (2007a), S. 89. Die Begriffe Anwendungsarchitektur, Applikationsarchitektur und Anwendungssystemarchitektur werden im Folgenden synonym verwendet.

257 Vgl. Klempt/Werners (2009), S. 311; Mantel/Schissler (2002), S. 171. Im Rahmen einer empirischen Analyse verdeutlichen Beimborn et al., dass die IT einen Einfluss auf die Gesamtprozessleistung aufweist. Vgl. BEIMBORN ET AL. (2006), S. $332 \mathrm{ff}$. Vgl. hierzu auch Heuser/Lacher/Perlmann (2007), S. 21.

259 Zur Abgrenzung der Begriffe Anwendungsarchitektur und IS-Architektur vgl. Abschnitt 3.2.1. 
te und interoperable IS-Architekturen zu schaffen, hat einen hohen Stellenwert, da das Zusammenspiel von Anwendungssystemen in einer IS-Architektur einen Schlüsselfaktor für die Gestaltung einer integrierten Informationsverarbeitung darstellt, wie sie beispielsweise im Rahmen des Supply Chain ManagementAnsatzes gefordert wird.

Aus der Perspektive der Wirtschaftsinformatik ,als Vermittler zwischen den betriebswirtschaftlichen Anforderungen der Unternehmen und den technischen Möglichkeiten der Informationsverarbeitung "260 interessiert in diesem Zusammenhang die Frage, auf welche Weise betriebliche Anwendungssysteme einerseits kosteneffizient gestaltet, andererseits auf Basis einer geeigneten ISArchitektur integriert werden können, um eine wirtschaftliche Informationsverarbeitung zu ermöglichen. ${ }^{261}$ Neben den unterschiedlichen Ansätzen zur Entwicklung von Anwendungssystemen existieren eine Fülle von Standards, Konzepten und Techniken, die sich in den einzelnen Phasen des Gestaltungsprozesses von Anwendungssystemen nutzen lassen. ${ }^{262}$ Trotz der Existenz verschiedener Lösungsansätze, die in den vergangenen Jahrzehnten zur Gestaltung integrierter Anwendungssysteme vorgeschlagen wurden, ${ }^{263}$ handelt es sich hierbei um ein Forschungsfeld, das nach wie vor eine hohe Relevanz in der Wirtschaftsinformatik besitzt. ${ }^{264}$

Von allen Konzepten, die sich mit der Frage beschäftigen, wie sich Anwendungssysteme und IS-Architekturen zur Unterstützung einer integrierten Informationsverarbeitung gestalten und/oder integrieren lassen, wird in den aktuellen Beiträgen unter dem Begriff Serviceorientierte Architektur bzw. Service Oriented Architecture (SOA) ein Ansatz diskutiert, der seit seiner begrifflichen Prägung eine besonders hohe Beachtung erfährt. ${ }^{265}$ In der wissenschaftlichen Aus-

260 Thome (1998), S. 964. Zum Verständnis der Wirtschaftsinformatik vgl. auch Laudon/ Laudon/Schoder (2006), S. 44.

261 Vgl. Glöckle (2007), S. 8 und 18; Heinrich/Klier/Bewernik (2006), S. 158. ТнОмЕ spricht in diesem Zusammenhang von einem integrierten System als „die Endstufe bei der Entwicklung wirtschaftlicher Informationsverarbeitungslösungen“. Thome (2007), S. 655 .

262 Vgl. Holten (2003), S. 41.

263 Einen Überblick über verschiedene Generationen von Integrationslösungen präsentieren Samtleben/Stadlbauer/Hess (2006), S. 88ff.; Hansen/Neumann (2005), S. 529ff.; Kink (2008), S. 359.

264 Diese Einschätzung wird auch von AIER/SCHÖNHERR geteilt, nach deren Auffassung die Integration und Interoperabilität der Infrastrukturen der von einer Unternehmung eingesetzten IT ein weiterhin ungelöstes Problemfeld darstelle. Vgl. Aier/Schönherr (2006), S. 188.

265 Vgl. Eymann/Winter (2008), S. 70; Mattern (2003), S. 34; Klesse/Wortmann/Schelp (2005), S. 259; Durst/Daum (2007), S. 18. Den Ausführungen von LiEBHART zufolge, wurde der Begriff einer SOA im Jahr 1996 von der IT-Marktforschungsunternehmung GARTNER RESEARCH geprägt. Vgl. Liebhart (2007), S. 6. 
einandersetzung sowie mit Blick auf die bisherigen Erfahrungsberichte erweisen sich die Erwartungshaltungen und die Einschätzungen zu SOA als vielfältig. Der Anspruch des vorliegenden Kapitels richtet sich daher auf das Ziel, die in der Literatur existierenden Aspekte zum verhältnismäßig jungen Forschungsgebiet der SOA zu einem integrierten Verständnis zusammenzuführen.

Der Aufbau des vorliegenden Kapitels gliedert sich wie folgt: Abschnitt 3.1 widmet sich zunächst einer Zustandsbeschreibung der betrieblichen Applikationslandschaft, die heute in vielen Unternehmungen vorherrscht. In diesem $\mathrm{Zu}$ sammenhang werden die Treiber vorgestellt, die in jüngster Zeit zu einem wachsenden Interesse an SOA geführt haben. Das Ziel von Abschnitt 3.2 liegt darin, die in den diversen Beiträgen thematisierten Merkmale einer SOA zu drei Sichtweisen zusammenzufassen. Im Anschluss widmet sich Abschnitt 3.3 der Frage, was unter dem Begriff des Softwareservices bzw. des Softwaredienstes zu verstehen ist und welche Prinzipien zur Entwicklung und Nutzung derartiger Dienste eingehalten werden müssen. Mit Blick auf die Implementierungsmöglichkeiten einer SOA thematisiert Abschnitt 3.4 die Technologie der Web Services. ${ }^{266}$ Aufbauend auf diesen Grundlagen wird in Abschnitt 3.5 ein integriertes Begriffsverständnis einer SOA erarbeitet.

\subsection{Integrierte Informationsverarbeitung im Kontext aktueller SOA- Treiber}

Die Erwartungen, die mit dem Betrieb einer SOA in der Literatur geäußert werden, richten sich auf die Minimierung der Defizite heutiger Anwendungssysteme, die eine effiziente und effektive integrierte Informationsverarbeitung verhindern. Ausgehend vom Begriff der integrierten Informationsverarbeitung in Abschnitt 3.1.1 thematisiert Abschnitt 3 den Zustand der betrieblichen Anwendungssysteme, wie er aktuell in vielen Unternehmungen zu beobachten ist. Daraufhin setzt sich Abschnitt 3.1.3 mit der Fragestellung auseinander, welche Treiber identifiziert werden können, die in der heutigen Unternehmenspraxis eine integrierte Informationsverarbeitung gefährden und damit maßgeblich zu einem wachsenden Interesse an SOA beigetragen haben.

\subsubsection{Begriff der integrierten Informationsverarbeitung}

In Anlehnung an GABRIEL/RÖHRS ist unter Informationsverarbeitung jeder Vorgang zu verstehen, der die Erfassung, Speicherung, Übertragung oder Trans-

266 In der Literatur werden neben der Schreibweise „Web Service“ auch die Varianten „Webservice“, „WebService“, „Web service“ und „Web-Service“ verwendet. Vor dem Hintergrund ihres verbreiteten Gebrauchs orientieren sich die folgenden Ausführungen an der Schreibweise „Web Service“. 
formation von Daten zum Gegenstand hat. ${ }^{267} \mathrm{Da}$ diese Vorgänge sequentiell ablaufen können, wird der daraus resultierende Prozess als Informationsverarbeitungsprozess bezeichnet. Die verschiedenen Fragestellungen, die sich mit der Integration der Informationsverarbeitung auseinandersetzen, thematisieren im Kern, inwieweit sich die verschiedenen Informationsverarbeitungsvorgänge und -ressourcen koordinieren bzw. zusammenfügen lassen. Dabei liegt diesen Fragestellungen ein Integrationsbegriff zugrunde, der aus der Perspektive der Wirtschaftsinformatik für die Verknüpfung von Menschen, Aufgaben und Technik steht. 268 Ziel der Integrationsbemühungen ist es, die unternehmungsbedingten Funktions-, Prozess- und Abteilungsgrenzen aufzuheben oder zumindest zurückzudrängen. In diesem Zusammenhang lassen sich verschiedene Perspektiven bzw. Typen einer integrierten Informationsverarbeitung unterscheiden. Im Fokus stehen dabei Integrationsgegenstände, die sich aus einer technischen Perspektive betrachten lassen. ${ }^{269}$ Abbildung 10 liefert eine Übersicht über häufig genannte Integrationsdimensionen. ${ }^{270}$

Vor dem Hintergrund der Aufbauorganisation einer Unternehmung lässt sich eine horizontale Integration von einer vertikalen Integration unterscheiden. Im Fokus dieses Integrationstyps stehen die betrieblichen Funktionen, die von bzw. in einer Unternehmung erbracht werden. Während eine horizontale Integration die funktionsübergreifende Verknüpfung der Administrations- und Dispositionssysteme entlang der betrieblichen Wertschöpfungskette zum Gegenstand hat, umfasst die vertikale Integration die Datenversorgung der Planungs- und Kontrollsysteme aus den Administrations- und Dispositionssystemen. ${ }^{271}$

Mit Blick auf die Integrationsreichweite steht der Möglichkeit einer innerbetrieblichen Integration die Variante einer zwischenbetrieblichen Integration gegenüber. ${ }^{272} \mathrm{Im}$ Gegensatz zur innerbetrieblichen Integration erfolgt bei der zwi-

Vgl. Gabriel/Röhrs (1995), S. 4.

$268 \mathrm{Vgl}$. Herden et al. (2006), S. 11. Welche Bedeutung diese drei Faktoren z. B. für die Zwecke einer integrierten Datenverarbeitung im Finanz- und Rechnungswesen haben, verdeutlicht Heinrich (2002), S. 45. AIER/WINTER bemerken in diesem Zusammenhang, dass die Wirtschaftsinformatik als interdisziplinäres Forschungsfach prädestiniert ist für Integrationsfragestellungen. Vgl. Aier/Winter (2009), S. 1.

269 Wie HERDEN ET AL. ausführen, lässt sich mit Blick auf den interdisziplinären Charakter der Wirtschaftsinformatik die Integration von IS nicht nur aus einer technischen sondern auch aus einer organisatorischen Perspektive betrachten. Vgl. Herden et al. (2006), S. 11.

270 Eine erweiterte Auflistung der in Abbildung 10 präsentierten Integrationsdimensionen liefern Fink/Schneidereit/Voß (2005), S. 212.

271 Vgl. Herden et al. (2006), S. 15.

272 Vgl. Herden et al. (2006), S. 16. 
schenbetrieblichen Integration die Verknüpfung der Informationsverarbeitungsressourcen über Unternehmungsgrenzen hinweg.

\begin{tabular}{|c|c|c|c|c|}
\hline Integrationsdimensionen & \multicolumn{4}{|c|}{ Ausprägungen } \\
\hline Integrationsrichtung & \multicolumn{2}{|c|}{ Horizontal } & \multicolumn{2}{|c|}{ Vertikal } \\
\hline Integrationsreichweite & \multicolumn{2}{|c|}{ Innerbetrieblich } & \multicolumn{2}{|c|}{ Zwischenbetrieblich } \\
\hline Integrationsobjekt & Geräte & Prozesse & Funktionen & Daten \\
\hline Automatisierungsgrad & \multicolumn{2}{|c|}{ Vollautomatisch } & \multicolumn{2}{|c|}{ Teilautomatisch } \\
\hline Informationstypen & Daten & Texte & Video & Audio \\
\hline
\end{tabular}

Abbildung 10: Ausgewählte Integrationsdimensionen

Eine weitere gebräuchliche Unterscheidung trennt die Integrationstypen Vorgangsintegration bzw. Prozessintegration, Datenintegration, Funktionsintegration und Geräteintegration. Bei der Prozessintegration wird angestrebt, Prozesse oder Vorgangsketten zu verknüpfen und ihre automatisierte Ausführung zu ermöglichen. Bei der Datenintegration ist es das Ziel, Datenbestände anwendungsübergreifend bereitzustellen. ${ }^{273}$ In Analogie zur Prozessintegration fokussiert die Funktionsintegration die Koordination der zum Einsatz kommenden Funktionen. Funktionen, die in einem Programm definiert werden können, transformieren Eingangsdaten in Ausgangsdaten nach einem vorgegeben Schema. ${ }^{274}$ Bei der Geräteintegration wird hingegen die Fragestellung thematisiert, wie sich eine gemeinsame Nutzung von Hardware-Komponenten koordinieren und durchführen lässt.

Ein weiteres wichtiges Abgrenzungsmerkmal der verschiedenen Integrationsmöglichkeiten ist der Automatisierungsgrad. In diesem Zusammenhang lässt

273 Vgl. Ließmann/Kaufmann/Schmitzer (1999), S. 12.

274 Bei einem Programm handelt es sich um eine Abfolge von Anweisungen, die die Verarbeitung von Daten auf einem Rechner steuern. Vgl. Laudon/Laudon/Schoder (2006), S. 35. Da in einem Programm Funktionen verwendet werden können, ist die Funktionsintegration gleichzeitig Basis für eine Programmintegration. Abzugrenzen ist eine Funktion von einer Aufgabe. Funktionen lassen sich in einem Programm vollautomatisiert ausführen, während eine Aufgabe von einem Subjekt ausgeführt wird und sich entweder gar nicht oder nur teilweise automatisieren lässt. Eine partielle Automatisierung ist gegeben, wenn Teilaufgaben durch den Einsatz von Programmen unterstützt werden. Vgl. Herden et al. (2006), S. 16. 
sich eine vollautomatische von einer teilautomatischen Integration unterscheiden. Eine vollautomatische Informationsverarbeitung ist gegeben, wenn sich verschiedene Aktivitäten eines Prozesses gegenseitig anstoßen, ohne dass eine menschliche Interaktion erfolgt. Im Vergleich zu dieser Integrationsart liegt bei einer teilautomatischen Integration eine menschliche Interaktion bei der Ausführung eines Prozesses vor.

Neben den bisher präsentierten Integrationsformen können auch die verschiedenen Informationstypen Gegenstand einer integrierten Informationsverarbeitung sein. Eine integrierte Informationsverarbeitung ist beispielsweise gegeben, wenn verschiedene Informationsdarstellungen und Medien, wie formatierte Daten, Texte, Grafiken, Video- und Audiosequenzen, aufeinander abgestimmt werden, um z. B. einen bestimmten Sachverhalt mit Hilfe eines aussagekräftigen Berichts zu vermitteln. ${ }^{275}$

\subsubsection{Betriebliche Anwendungssysteme - State of the Art}

Allgemein ist eine mangelnde Ausrichtung der IT an die Gesamtunternehmung zu bemerken, die zu einer Reihe von Problemfeldern geführt hat.276 Wie MELZER ET AL. ausführen, sind viele Unternehmungen derzeit mit IT-Systemen konfrontiert, die sich als historisch gewachsene Systeme in den vergangenen Jahren isoliert von anderen Systemen entwickelt haben und folglich heute als so genannte „Insellösungen“ betrieben werden. ${ }^{277}$ Als begriffliche Alternativen für derartige Systeme finden sich die Bezeichnungen „Applikationssilos“278 und „IT-Säulen“279. Zur Entwicklung dieser Systeme wurden in den vergangenen Jahren hohe Investitionen aufgewendet. 280

Ursprünglich als Systeme zur Abwicklung von kleinen sowie speziellen Aufgaben entwickelt und auf die verschiedenen Fachbereiche einer Unternehmung zugeschnitten, haben diese Systeme im Laufe ihrer Nutzung an Funktionalität und Komplexität hinzu gewonnen.281 Vor dem Hintergrund der Restriktion, dass in den vergangenen Jahren - ebenso wenig wie in der heutigen Praxis keine Softwaresysteme als Gesamtpaket verfügbar waren, die alle Funktionen

275 Die integrierte Verarbeitung unterschiedlicher Informationsdarstellungen und Medien wird als Multimedia bezeichnet. Vgl. Hansen/Neumann (2005), S. 7.

276 Vgl. Gericke/Stutz (2006), S. 362.

277 Vgl. Melzer et al. (2007), S. 28.

278 Vgl. Höß et al. (2007), S. 39; Schelp/Winter (2007), S. 43.

279 Vgl. Melzer et al. (2007), S. 28.

$280 \mathrm{Vgl.} \mathrm{Kossmann/Leymann} \mathrm{(2004),} \mathrm{S.} 117$.

281 Vgl. Samtleben/Stadlbauer/Hess (2006), S. 87. Komplexität lässt sich als Ergebnis technischer und geschäftlicher Entscheidungen auffassen, die zu einer negativen Entwicklung der Applikationsarchitektur beigetragen haben. Zur Auflistung einiger komplexitätssteigernder Faktoren vgl. Gimnich (2007), S. 69. 
einer Unternehmung abdeckten, wurde entweder für jede zu unterstützende Funktion ein separates Softwaresystem eingesetzt oder die betreffende Funktion in dem bereits existierenden System individuell entwickelt. ${ }^{282}$ Auf diese Weise ließen sich die Informationsverarbeitungsaufgaben in den verschiedenen Geschäftsfunktionen einer Unternehmung wie z.B. Beschaffung, Vertrieb und Marketing, Fertigung und Produktion, Finanz- und Rechnungswesen sowie Personalwesen IT-gestützt bewältigen. ${ }^{283}$ Während bei der Auswahl sowie der Entwicklung von Software das Interesse vieler IT-Abteilungen auf die Funktionalität und die Architektur des Systems gerichtet war, spielte die Integration dieser Anwendungen zunächst eine untergeordnete Rolle. ${ }^{284}$

Die heute zu beobachtende Konsequenz aus der skizzierten Entwicklung ist, dass in den Unternehmungen sehr viele spezialisierte Softwaresysteme betrieben werden, 285 die nicht nur instransparent, sondern auch redundant sind. ${ }^{286}$ Die Softwaresysteme zeichnen sich dabei nicht nur durch ihren spezifischen Anwendungsbereich aus, für den sie gestaltet wurden, 287 sondern auch durch die Heterogenität der bereitgestellten Schnittstellen und der verwendeten Programmiersprachen. Hierbei stehen ,neue“ Programmiersprachen, die wie beispielsweise im Fall von Java oder C++ objektorientierte Konzepte unterstützen, ,ältere" Programmiersprachen gegenüber, die den ersten Sprachgenerationen zuzuordnen sind. ${ }^{288}$ Auch wenn letztere insbesondere zum Betrieb der Altsysteme notwendig sind, ${ }^{289}$ haben sie heute nur eine untergeordnete Relevanz für eine produktive, an den Qualitätsstandards ausgerichtete Softwareentwicklung. 290 Die Heterogenität der Anwendungssysteme stützt sich nicht nur auf die verwendeten proprietären Softwaresysteme, sondern auch auf die verschiedenen Hardwareplattformen, die wie die Softwaresysteme von verschiedenen kom-

282

283

284

Vgl. Kossmann/Leymann (2004), S. 117.

$\mathrm{Zu}$ den Geschäftsfunktionen vgl. Laudon/Laudon/Schoder (2006), S. 37.

Vgl. Samtleben/Stadlbauer/Hess (2006), S. 87.

Vgl. Klesse/Wortmann/Schelp (2005), S. 259; Kossmann/Leymann (2004), S. 117; Samtleben/Stadlbauer/Hess (2006), S. 87.

Vgl. Gericke/Stutz (2006), S. 362.

In Abhängigkeit vom Verwendungszweck lassen sich die Kategorien Systemsoftware, Entwicklungssoftware und Anwendungssoftware unterscheiden. Vgl. Hansen/Neumann (2005), S. $163 f$.

Eine Übersicht zu den Programmiersprachen verschiedener Generationen präsentieren Fink/Schneidereit/Voß (2005), S. 32ff. Einen Überblick über die derzeit nachgefragtesten Programmiersprachen liefert der Programmiersprachenpopularitätsindex, der von der Unternehmung TIOBE veröffentlicht wird. Vgl. Tiobe (2008).

$\mathrm{Zu}$ den Eigenschaften von Altsystemen vgl. Abschnitt 3.1.3.1.

Die wichtigsten Qualitätsmerkmale von Software werden von POMBERGER/BLASCHEK diskutiert. Vgl. Pomberger/Blaschek (1993), S. $9 \mathrm{ff}$. 
merziellen und nichtkommerziellen Herstellern angeboten werden.291 Nach der Einschätzung von KLESSE/WORTMANN/SCHELP ist trotz der ansteigenden Verbreitung und Verwendung von Standardsoftware auch in den kommenden Jahren nicht zu erwarten, dass die heterogenen Systemlandschaften abgelöst werden. 292

Im Ergebnis hat die bisher beschriebene Entwicklung in den vergangenen Jahren zu einer vertikal organisierten IT-Landschaft geführt, die, wie in der Abbildung $11 \mathrm{zu}$ erkennen ist, aus einer Vielzahl isolierter Anwendungssysteme besteht. 293 Derartige Anwendungssysteme verfügen über einen eigenen Datenbestand, der sich mit Hilfe eines vorgegebenen Vorrats an Funktionen verarbeiten lässt. Ferner zeichnen sich isolierte Anwendungssysteme durch eine eigene Prozesssteuerung aus. Zur Prozesssteuerung stellen die Systeme ihren Anwendern eine eigene Benutzungsschnittstelle in Form eines Klienten (Client) oder einer grafischen Benutzungsoberfläche, die auch als Graphical User Interface (GUI) bezeichnet wird, zur Verfügung.

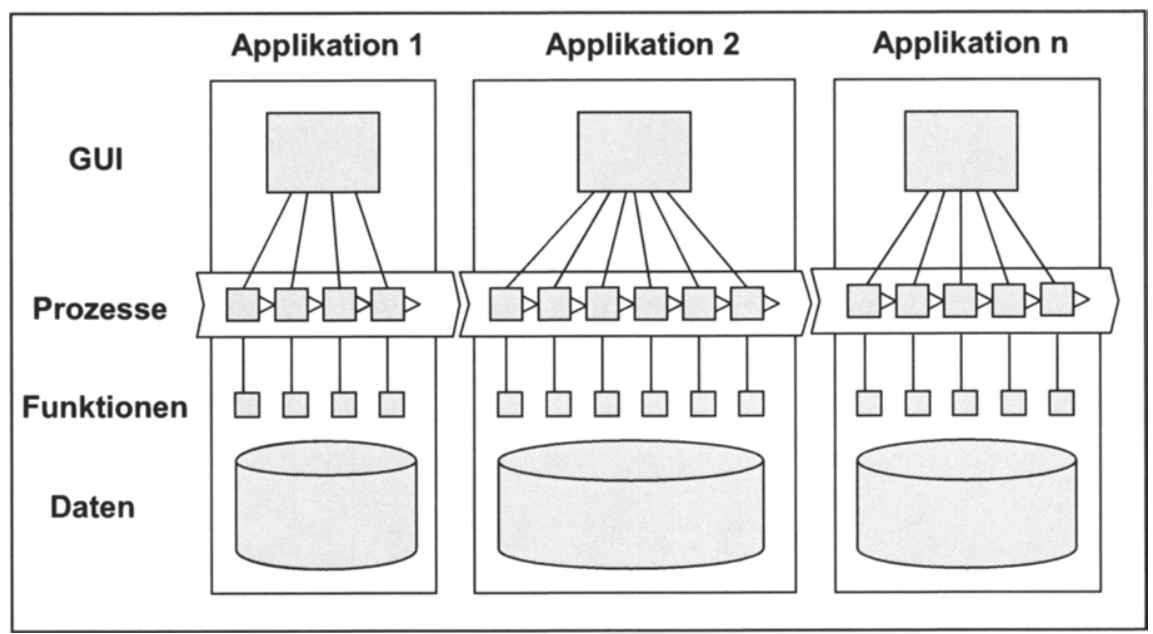

Abbildung 11: Applikationssilos 294

291 Den Einsatz veralteter und teilweise proprietärer Technologien bemängeln z. B. Höß et al. (2007), S. 39.

292 Vgl. Klesse/Wortmann/Schelp (2005), S. 259.

293 Vgl. Rieks (2006), S. 6.

294 In Anlehnung an Melzer et al. (2007), S. 29. 
Um betriebswirtschaftliche Konzepte wie z. B. das Supply Chain Management umzusetzen, ist es erforderlich, die heterogenen Anwendungssysteme zu koppeln und mit den Geschäftsprozessen zu verknüpfen. ${ }^{295}$ Mit Hilfe des Einsatzes integrierter betrieblicher Anwendungssysteme wird dabei das Ziel verfolgt, organisatorische und informationstechnische Verbundeffekte zu realisieren. ${ }^{296}$ Die mit diesem Ziel durchgeführten Integrationsprojekte hatten zur Folge, dass die Applikationen gewachsen sind und durch die gestiegene Anzahl an Funktionen eine beträchtliche Komplexität erreicht haben. ${ }^{297}$ Darüber hinaus verfügen die heutigen Anwendungssysteme über eine hohe Anzahl an Schnittstellen zu anderen Applikationen, wodurch vielfältige Abhängigkeitsbeziehungen resultieren, die zu einem Gebilde geführt haben, das heute auch als „SpaghettiArchitektur" bezeichnet wird.298 Im Ergebnis ist festzuhalten, dass die historisch gewachsenen IT-Architekturen nicht nur die Komplexität, ${ }^{299}$ sondern auch die Unübersichtlichkeit der Gesamtarchitektur einer Unternehmung forciert haben. 300

\subsubsection{Treiber für das Interesse an SOA}

Als Folge der im vorherigen Abschnitt skizzierten Entwicklung lassen sich Defizite identifizieren, die derzeit ein Problemfeld in den IT-Abteilungen vieler Unternehmungen darstellen. Die zu erläuternden Problembereiche lieferten in den vergangenen Jahren den Ausgangspunkt für die wissenschaftliche Auseinandersetzung mit neuen fachlichen Konzepten und technischen Ansätzen zur Entwicklung sowie zur Integration von Anwendungssystemen. Der prominenteste Vertreter unter diesen Vorschlägen wird derzeit unter dem Themenfeld SOA diskutiert.

Die Ausführungen dieses Abschnitts verfolgen das Ziel, die in der Literatur viel zitierte Bedeutung von SOA anhand geeigneter Einflussfaktoren zu systematisieren. Als Ausgangspunkt hierfür können die Treiber zu Rate gezogen werden, die im Zusammenhang mit dem Einsatz einer SOA in den diversen Literaturbeiträgen genannt wurden. Zur Identifizierung der relevanten Treiber für das Interesse an SOA wurde eine Analyse ausgewählter Beiträge durchgeführt, deren Ergebnisse überblicksartig in Abbildung 12 dargestellt sind. Um die Er-

Vgl. Holten (2003), S. 41.

Vgl. Müller/Hess (2006), S. 109.

Dem Verständnis von HANSEN/NEUMANN folgend, steht der Komplexitätsbegriff für den Umfang der exakten Beschreibung aller Einzelheiten eines IS und ihrer Abhängigkeiten voneinander. Vgl. Hansen/Neumann (2005), S. 169.

Vgl. Holtschke/Heier/Hummel (2009), S. 109.

Vgl. Schelp (2007), S. 144.

Vgl. Holtschke/Heier/Hummel (2009), S. 109. 
gebnisse in einer anschaulichen Weise zu präsentieren, wurden die Treiber stichwortartig zusammengefasst und den jeweiligen Autoren zugeordnet.

\begin{tabular}{|c|c|c|c|c|c|c|}
\hline $\begin{array}{l}\text { Beimbornwoltzel } \\
\text { (2003) }\end{array}$ & \multicolumn{2}{|c|}{$\begin{array}{c}\text { Inner- und zwischenbetriebliche } \\
\text { Integration von Arwendungssystemen }\end{array}$} & \multicolumn{2}{|c|}{ Automatischer Datenaustausch } & \multicolumn{2}{|r|}{ Prozessautomatisierung } \\
\hline $\begin{array}{l}\text { Durstidaum } \\
\text { (2007) }\end{array}$ & \multicolumn{2}{|l|}{$\begin{array}{l}\text { Schnelle Anpessung an } \\
\text { Marktveranderungen }\end{array}$} & \multicolumn{2}{|c|}{ Wiederverwendbarkeit } & \multicolumn{2}{|c|}{$\begin{array}{c}\text { Flexibilisienung von } \\
\text { Geschaftsprozessen / Unterstutzung } \\
\text { neuer Geschaftsprozesse }\end{array}$} \\
\hline $\begin{array}{l}\text { Hose/HummNoB } \\
\text { (2006) }\end{array}$ & \multicolumn{3}{|c|}{ Hohe Kosten des T-Betriebs } & \multicolumn{3}{|c|}{$\begin{array}{l}\text { Flexibilisierung von Gescháftsprozessen / Unterstützung } \\
\text { neuer Geschätsprozesse }\end{array}$} \\
\hline $\begin{array}{c}\text { Heutschileugnerl } \\
\text { Osterle } \\
(2006)\end{array}$ & $\begin{array}{c}\text { Unzulangliche bzw. fehlende } \\
\text { Unterstutzung bestehender } \\
\text { Geschaftsproezsse }\end{array}$ & \multicolumn{2}{|c|}{$\begin{array}{l}\text { Mangelhafte Flexibilitat } \\
\text { beim Umsezen neuer } \\
\text { Geschafts- bzw. } \\
\text { Markanforderungen } \\
\end{array}$} & \multicolumn{2}{|c|}{$\begin{array}{l}\text { Hohe Kosten des } \pi \text { - } \\
\text { Betriebs }\end{array}$} & $\begin{array}{c}\begin{array}{c}\text { Probleme bei notwendigen } \\
\text { technischen } \\
\text { Modemisierungen in der } \mathrm{rT} . \\
\text { Architektur }\end{array} \\
\end{array}$ \\
\hline $\begin{array}{l}\text { Hoß ot al. } \\
(2007)\end{array}$ & \multicolumn{3}{|c|}{ Móglichst gute Prozessunterstützung durch die $\Pi$} & \multicolumn{3}{|c|}{ Flexible Reaktion aut Prozessànderungen } \\
\hline $\begin{array}{l}\text { Leyking/Ziemann/Loos } \\
\text { (2006) }\end{array}$ & \multicolumn{3}{|c|}{$\begin{array}{l}\text { Engere Verknupfung der IT-Systeme mit betrieblichen } \\
\text { Funktionen und Geschaftsprozessen }\end{array}$} & \multicolumn{3}{|c|}{ Flexiblere und bessere Steuerung der IT-Systeme } \\
\hline $\begin{array}{l}\text { RlehteriHaller/Sehrey } \\
\text { (2005) }\end{array}$ & \multicolumn{2}{|c|}{ Hohe Kosten des IT-Betriebs } & \multicolumn{2}{|c|}{$\begin{array}{l}\text { Flexible Reaktion auf Ānderungen in } \\
\text { der Unternehmensstruktur }\end{array}$} & Gesc & $\begin{array}{l}\text { Flexibilisierung von } \\
\text { tsprozessen / Unterstutzung } \\
\text { Jer Geschaftsprozesse }\end{array}$ \\
\hline
\end{tabular}

\section{Abbildung 12: Literaturauswertung zu den Treibern einer SOA}

Aus den Nennungen lassen sich zwei Treiberkategorien identifizieren, die das Interesse an SOA forciert haben. Während Abschnitt 3.1.3.1 die Kosten der Systementwicklung und der Systemintegration in den Vordergrund rückt, wird in Abschnitt 3.1.3.2 die mangelnde IT-Unterstützung von Geschäftsprozessen als zweite Treiberkategorie thematisiert. 301

\subsubsection{Kosten des IT-Betriebs}

Vor dem Hintergrund sinkender IT-Budgets besitzt die Anforderung, die Kosten für die Entwicklung, den Betrieb und die Wartung heterogener Anwendungssysteme zu reduzieren, eine wichtige Herausforderung für die IT-Abteilungen der Unternehmungen. ${ }^{302}$ Mit Blick auf die in Abschnitt 3 beschriebenen Eigenschaften einer funktional angeordneten IT-Landschaft einer Unternehmung sind einem kostengünstigen Betrieb der IT-Landschaft einer Unternehmung enge

301 Dass die Erwartungshaltung an den Aufbau einer SOA sich auf die Flexibilität der Geschäftsprozesse und die Reduktion von IT-Kosten richtet, wird ebenfalls bei HuMM/JuWIG deutlich. Vgl. Humm/Juwig (2006), S. 1.

302 Vgl. Hofmann (2003), S. 27. Da Unternehmungen nach LANKES ET AL. ohnehin einem wachsenden Kostendruck ausgesetzt sind, gewinnt diese Anforderung umso mehr an Bedeutung. Vgl. Lankes et al. (2008), S. 12. 
Grenzen gesetzt. ${ }^{303}$ Darüber hinaus wird gefordert, die in Abschnitt 3 thematisierte Heterogenität in Bezug auf die eingesetzte Software und Hardware sowie die implementierten Schnittstellen zu überwinden. Hinweise für diese Feststellung lassen sich in den Nennungen der Abbildung 12 entnehmen. Aus den Nennungen geht hervor, dass die ,hohen Kosten des IT-Betriebs“, die „flexiblere und bessere Steuerung der IT-Systeme“, die ,inner- und zwischenbetriebliche Integration von Anwendungssystemen“ sowie die „Probleme bei notwendigen technischen Modernisierungen in der IT-Architektur" wichtige Treiber darstellen, die einen effizienten Betrieb der IT-Landschaft einer Unternehmung gefährden und sich daher zu einer Treiberkategorie zusammenfassen lassen. ${ }^{304}$

Wie HÖß ET AL. in diesem Zusammenhang bemerken, fehlt es den bestehenden Systemen an Offenheit und Flexibilität, um die an sie gestellten Anforderungen zu bewältigen. ${ }^{305}$ Flexibilität kann dabei als die Eigenschaft eines Systems verstanden werden, auf sich abzeichnende Änderungen im System oder im Umfeld des Systems ex ante in einer sinnvollen Weise reagieren zu können. ${ }^{306}$ Damit richtet sich Flexibilität auf die Fähigkeit einer Applikation, geänderte Bedarfe zur Informationsverarbeitung zu befriedigen oder sich sonstigen Veränderungen im Umfeld der Applikationslandschaft anzupassen. 307

Einen gewichtigen Kostenblock, der aus der Inflexibilität der betriebenen IS resultiert, bilden die Wartungskosten. In der Softwareentwicklung besitzt die Wartungsphase eine wichtige Bedeutung, da sie nicht nur den längsten, sondern i. d. R. auch den teuersten Abschnitt im Lebenszyklus eines Softwaresystems repräsentiert. ${ }^{308}$ Abbildung 13 verdeutlicht, dass die Kosten, die über den gesamten Lebenszyklus eines Softwaresystems für die Wartung anfallen, in den vergangenen 50 Jahren stetig gewachsen sind. Im Vergleich zu den Kosten, die für die reine Softwareentwicklung sowie für die Beschaffung und Benutzung der Hardware entstehen, machen die Wartungskosten heute mehr als $70 \%$ der Gesamtkosten aus. Gleichzeitig ist festzustellen, dass Softwareprojekte mit einem oft zu geringen Wartungsbudget auskommen müssen. ${ }^{309}$

303

304

305

306

307

Vgl. Holtschke/Heier/Hummel (2009), S. 109.

Zum Begriff der IT-Architektur vgl. die Ausführungen in Abschnitt 3.1.3.2.

Vgl. Höß et al. (2007), S. 39.

Vgl. Müller (2007a), S. 91; Schelp (2007), S. 147.

Vgl. Müller (2007a), S. 91. Abzugrenzen ist der Flexibilitätsbegriff vom Terminus der Geschwindigkeit, der auf die Reaktionsfähigkeit der betrieblichen Anwendungssysteme abzielt, nicht vorhergesehenen Entwicklungen bzw. Änderungen ohne eine signifikante zeitliche Verzögerung begegnen zu können. Vgl. Schelp (2007), S. 147.

Vgl. Gluchowski/Gabriel/Chamoni (1997), S. 132.

Vgl. Gimnich (2007), S. 69. 


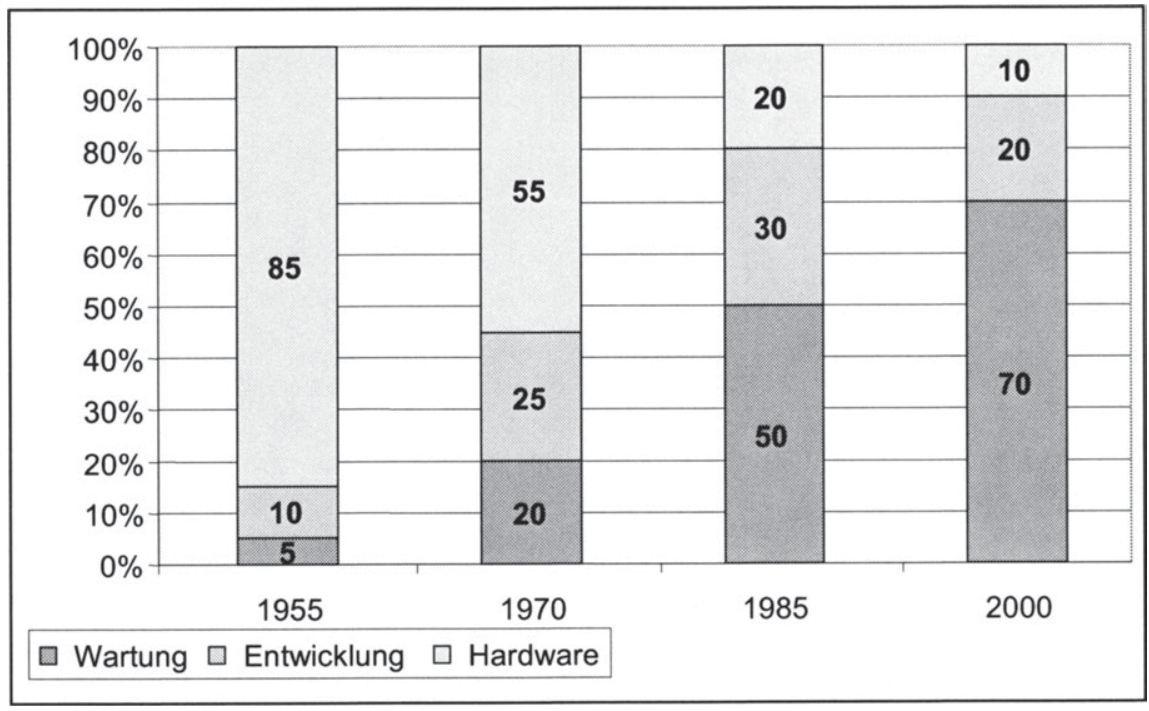

\section{Abbildung 13: Steigende Wartungskosten 310}

Neben den Kosten infolge inflexibler IS ergeben sich weitere Aufwendungen, die bei der Integration der betrieblichen Anwendungssysteme anfallen. 311 Mit Blick auf die Notwendigkeit, i. d. R. eine Vielzahl von Applikationen zu integrieren, liegt ein wichtiger Kostenblock, der im Rahmen eines derartigen Projekts aufzuwenden ist, in der Wartung und Weiterentwicklung der Schnittstellen der $\mathrm{zu}$ integrierenden Systeme. ${ }^{312}$ Als Ursache für dieses Defizit ist zu bemerken, dass mit Blick auf die Vielfalt der zur Verfügung stehenden Kommunikationsmechanismen bzw. -protokolle und modellierten Datenformate fehlende Standards eine kosteneffiziente Wartung und Wiederverwendbarkeit der Schnittstellen verhindert haben. 313

310 In Anlehnung an Mas y Parareda/Pizka (2007), S. 60.

311 Dass die Integration betrieblicher Anwendungssysteme heute einen bedeutenden Kostenfaktor darstellt, wird an den Kosten deutlich, die Fortune-1000-Unternehmungen für derartige Projekte aufwenden. Nach KAIB investierten diese jährlich mehr als $100 \mathrm{Mrd}$. US \$ für Integrationsprojekte. Vgl. Kaib (2002), S. 1.

312 Vgl. hierzu Höß et al. (2007), S. 40. Wird ein klassisches sequentielles Vorgehensmodell zugrunde gelegt, bildet die Wartung i. d. R. die abschließende Phase des Softwareprojekts. Ihr gehen die Phasen Problemanalyse, Entwurf, Implementierung, Funktionsund Leistungsüberprüfung, Installation und Abnahme voraus. Vgl. Gabriel/Röhrs (1995), S. 122ff. Auf die Bedeutung einer effizienten und effektiven Schnittstellengestaltung für Unternehmungen verweisen auch Hirsch/Sorg (2006), S. 428.

313 Vgl. Rieks (2006), S. 6. 
Dass die Wartung der Schnittstellen eine ressourcenintensive Aufgabe darstellt, wird auch durch die Einschätzung von KLESSE/WORTMANN/SCHELP gestützt, die darauf verweisen, dass 35 bis $60 \%$ des IT-Budgets für die Erstellung und Wartung der Schnittstellen verbraucht werden. ${ }^{314}$ Weiterhin beträgt der Aufwand für das Schnittstellendesign und die -implementierung ca. 30\% der Zeit bei Softwareentwicklungsprojekten. ${ }^{315}$ Als großes Problem erweist sich in diesem Zusammenhang die Existenz so genannter Punkt-zu-Punkt-Schnittstellen, die erstellt werden, um bedarfsweise bzw. ad hoc bestehende mit neuen Anwendungen zu verbinden. ${ }^{316}$ Das Ergebnis einer derartigen Vorgehensweise mündet in ein komplexes Anwendungsgeflecht und in schwer wartbare Abhängigkeiten.

Weitere Kosten, die zur Bewältigung der Heterogenität der Anwendungssysteme auftreten, lassen sich am besten am Beispiel der Altsysteme verdeutlichen, die auch als Legacy-Systeme bezeichnet werden. Auch wenn sich bisher keine allgemeine und etablierte Definition durchgesetzt hat, was unter einem LegacySystem zu verstehen ist, 317 herrscht Einigkeit, dass sich derartige Systeme als alte bzw. gewachsene Softwaresysteme auffassen lassen, deren Entwicklung viele Jahre zurückliegt. 318 Oftmals kommen im Rahmen von Altanwendungen auf Mainframes installierte, abteilungsübergreifende oder gar unternehmungsweite Datenbanksysteme zum Einsatz. ${ }^{319}$ Zur weiteren Präzisierung, was unter einem ,alten" Softwaresystem zu verstehen ist, werden in der vorliegenden Arbeit alle Softwaresysteme als Legacy-Systeme bezeichnet, die vor der Verbreitung der objektorientierten Programmierung entwickelt wurden. ${ }^{320}$ Eine weitere Konkretisierung dieser Auslegung erfolgt bei LIEBHART, dessen Auffassung nach Legacy-Systeme in Assembler oder einer früheren Version einer Drittgenerationssprache wie COBOL oder ALGOL geschrieben wurden. 321

Aufgrund ihres monolithischen und heterogenen Charakters ist eine Verknüpfung derartiger IS mit anderen Applikationen zudem nur unter Inanspruchnah-

Vgl. Klesse/Wortmann/Schelp (2005), S. 259.

Vgl. Klesse/Wortmann/Schelp (2005), S. 259.

Vgl. Gimnich (2007), S. 69.

Vgl. Liebhart (2007), S. 182.

Vgl. Mas y Parareda/Pizka (2007), S. 60.

Siehe hierzu das Beispiel bei Sunyaev et al. (2006), S. 31.

Diese Abgrenzung folgt der Sichtweise von SOMMERVILLE. Vgl. Sommerville (2001), S. 595. Eine Zäsur bildet in diesem Zusammenhang der Beginn der 1990-er Jahre, als C++ sich als dominierende Sprache der objektorientierten Programmierung durchgesetzt hat. Zur Geschichte der objektorientierten Softwareentwicklung vgl. Balzert (2000), S. 7ff.

Der Autor liefert darüber hinaus weitere „Indizien“ zur begrifflichen Präzisierung eines Legacy-Systems. Vgl. Liebhart (2007), S. 182. Das Akronym COBOL steht für „Common Business Oriented Language“". ALGOL stellt eine Abkürzung für die Programmiersprache „Algorithmic Language“ dar. 
me hoher Aufwendungen zu bewerkstelligen. ${ }^{322}$ Auch die Auslagerung von Prozessaktivitäten und damit die Einbindung externer IT-Dienstleister und deren Services führen beim Betrieb eines monolithischen Systems zu hohen Aufwendungen. ${ }^{323} \mathrm{Da}$ Legacy-Systeme sich i. d. R. aus einem Zusammenspiel diverser Anwendungssysteme und Programme zusammensetzen, ist davon auszugehen, dass diese Programme erst im Lauf der Nutzung eines Altsystems hinzugefügt oder geändert wurden. Dabei ist ebenfalls anzunehmen, dass bei der Änderung bzw. Modifikation der Legacy-Systeme eine Reihe von Anwendern und Entwicklern partizipiert haben, deren individuelle Anforderungen sowie Fachkenntnisse zu einer permanenten, unkontrollierten Weiterentwicklung der Systeme geführt haben.

Darüber hinaus ist zu konstatieren, dass notwendig gewordene Anpassungen und Veränderungen an den Systemen, die unter Einsatz verschiedener, z. T. veralteter Programmiersprachen mit unterschiedlichen Versionen durchgeführt wurden, oftmals ungenügend oder unzureichend dokumentiert wurden. ${ }^{324}$ Dieses Problem erweist sich als umso gravierender, je mehr unterschiedliche Programmierstile sowie Konzepte des Software Engineering bei der (Weiter-) Entwicklung eines derartigen Systems zur Anwendung kamen. In vielen Fällen ist bei der Verwendung von Legacy-Systemen nicht nur ein Mangel an Dokumentation des Quellcodes, sondern auch eine nicht vorhandene Spezifikation der Systeme festzustellen. Ebenso sind i. d. R. bei dem Betrieb von LegacySystemen Geschäftsabläufe sowie Geschäftsbeschreibungen an keiner Stelle festgehalten oder auffindbar. In diesem Zusammenhang erweist es sich als weitere Schwierigkeit, Mitarbeiter zu finden, die über entsprechende Programmierkenntnisse sowie eine Ausbildung im Umgang mit den eingesetzten Technologien, Programmiersprachen und Hardware-Systemen eines Legacy-Systems verfügen. 325

Neben den Kosten, die sich aus der Wartung der Schnittstellen sowie aus einer Verwendung unterschiedlicher Programmiersprachen ergeben, sind darüber hinaus Aufwendungen zu berücksichtigen, die für die Hardware derartiger Anwendungssysteme anfallen. Hierbei ist festzustellen, dass Legacy-Systeme i. d. R. auf Hardware-Komponenten von Großrechnern basieren, die in den

322 Monolithische Anwendungssysteme zeichnen sich dadurch aus, dass ihre Systemkomponenten eine untrennbare Einheit bilden und sich daher nur schwer bzw. unwirtschaftlich sowie unter Reduzierung der Softwarequalität herauslösen oder ersetzen lassen. I. d. R. setzen Monolithen auf einem proprietären Betriebssystem auf. Monolithische Anwendungssysteme wurden auf einem zentralen Großrechner (Mainframe) installiert. Eine Benutzerinteraktion erfolgte mit Hilfe von Terminals. Vgl. Herden et al. (2006), S. 25f.

Vgl. Braunwarth/Heinrich (2008), S. 100.

324 Vgl. Mas y Parareda/Pizka (2007), S. 60; Sommerville (2001), S. 591.

Vgl. Mas y Parareda/Pizka (2007), S. 60. 
meisten Fällen von den betreffenden Produktherstellern nicht mehr produziert oder unterstützt werden. Dies hat zur Konsequenz, dass Defekte an der Hardware sich im günstigsten Fall nur über teure Wartungsmaßnahmen beheben oder zumindest reduzieren lassen.

Mit Blick auf den ressourcenintensiven Betrieb einer heterogenen Applikationslandschaft ist festzustellen, dass die Aufwendungen, die für den Betrieb der IT-Landschaft anfallen, insbesondere die so genannte operationelleAgilität der Anwendungssysteme gefährden. ${ }^{326}$ Die operationale Agilität zielt auf die Fähigkeit einer Unternehmung ab, neue Geschäftspotenziale zu erkennen und diese zeitnah, exakt und kosteneffizient umzusetzen. Betroffen von den Anforderungen, eine operationale Agilität zu gewährleisten, sind insbesondere die ITAbteilungen, deren Arbeit darauf ausgerichtet ist, in den Unternehmungen eine entsprechende Applikationslandschaft mit dem Ziel zu betreiben, die Fachbzw. Anwendungsseite zu unterstützen. ${ }^{327}$

Dadurch, dass zur Überwindung der Heterogenität infolge historisch gewachsener, isolierter und monolithischer Anwendungssysteme beträchtliche Ressourcen seitens der IT-Abteilungen aufgewendet werden müssen, ist das Ziel gefährdet, die von der Fach- bzw. Anwendungsseite erwartete IT-Unterstützung in einer effizienten und effektiven Weise zu leisten. ${ }^{328}$

326 Allgemein lässt sich Agilität als Fähigkeit verstehen, sich an Veränderungen anzupassen. Vgl. Dorda/Steiert/Sellentin (2004), S. 200. Nach YUSUF/SARHADI/GUNASEKARAN lässt sich Agilität (agility) wie folgt definieren: „Agility is the successful exploration of competitive bases (speed, flexibility, innovation proactivity, quality and profitability) through the integration of reconfigurable resources and best practices in a knowledgerich environment to provide costumer-driven products and services in a fast changing market environment“". Yusuf/Sarhadi/Gunasekaran (1999), S. 37. Wie aus dem Definitionsvorschlag hervorgeht, lässt sich Agilität in die Teilziele Geschwindigkeit, Flexibilität, proaktive Innovation, Qualität und Profitabilität aufspalten. Die Erfüllung dieser Teilziele trägt dazu bei, Produkte und Dienstleistungen anzubieten, die sich an den Bedürfnissen der Kunden orientieren. Vor dem Hintergrund eines wissensintensiven, sich schnell ändernden Marktumfelds liegt den Autoren zufolge ein zentraler Erfolgsfaktor für diese Zielerreichung in der Integration rekonfigurierbarer Ressourcen. Während der Agilitätsbegriff von YUSUF/SARHADI/GUNASEKARAN aus einer allgemeingültigen Sicht betrachtet wird, untersuchen SAMBAMURTHY ET AL. die Unterstützungspotenziale, die die betrieblichen IS für die Agilität einer Unternehmung bergen. Die Autoren unterscheiden in diesem Zusammenhang die Agilitätstypen operationelle Agilität, kundensowie partnerorientierte Agilität. Vgl. Sambamurthy et al. (2003), S. 245f. Zur Bedeutung der kunden- sowie partnerorientierten Agilität vgl. Abschnitt 3.1.3.2. Vgl. Schelp/Winter (2007), S. 43.

328 Wie HÖв ET AL. in diesem Zusammenhang bemängeln, lassen sich infolge des erschwerten Einsatzes integrierter Systeme kein Mehrwert erzielen und keine Redundanzen vermeiden. Vgl. Höß et al. (2007), S. 39. 


\subsubsection{Mangelnde IT-Unterstützung von Geschäftsprozessen}

Die im vorherigen Abschnitt thematisierten Problemfelder für die operationelle Agilität der IS einer Unternehmung erweisen sich insbesondere als gravierend, wenn nicht einzelne isoliert betrachtete Funktionen, sondern funktionsübergreifende Geschäftsprozesse den Gegenstand einer IT-Unterstützung darstellen. ${ }^{329}$ Als verbindendes Glied zwischen der Strategie und der IT einer Unternehmung liegt in der Modellierung und Implementierung von Geschäftsprozessen ein wichtiger Faktor für den Erfolg einer Unternehmung. Ein Geschäftsprozess kann innerhalb einer Organisation und/oder organisationsübergreifend ausgestaltet sein, wobei sich die miteinander verknüpften Aktivitäten sequentiell und/oder parallel ausführen lassen. ${ }^{330}$

Wie sich den Nennungen zu den Treibern einer SOA entnehmen lässt, wird gefordert, die Funktionalität der IT stärker an den Geschäftsprozessen einer Unternehmung auszurichten. Da der Einschätzung von HEß zufolge die Mehrzahl der prozessrelevanten Daten in den IT-Systemen vorgehalten wird, ist es erforderlich, die Daten automatisiert und permanent von den Quellsystemen zu extrahieren. ${ }^{331}$ Darüber hinaus lässt sich die Forderung ableiten, mit Hilfe der extrahierten Daten den gesamten Geschäftsprozess - angefangen bei der Anfrage eines Kunden bis zur Auslieferung und zum Zahlungseingang - abzubilden sowie mittels der eingesetzten IT zu automatisieren. ${ }^{332}$

Bedenkt man, dass die zu unterstützenden Geschäftsprozesse einem immer schnelleren Wandel ausgesetzt sind, ${ }^{333}$ richtet sich ein weiteres wichtiges Augenmerk auf die Gestaltbarkeit sowie die Anpassungsfähigkeit der Geschäftsprozesse, die sich durch ein hohes Maß an Innovationspotenzial sowie Adaptivität auszeichnen sollen. 334 Aus der Zusammenstellung der Nennungen in Abbildung 12 geht hervor, dass sowohl die Anpassungsfähigkeit bestehender Geschäftsprozesse als auch die Gestaltbarkeit zukünftiger Geschäftsprozesse wichtige Treiber für das Interesse an SOA darstellen. SCHELP fügt dieser Feststellung hinzu, dass der geforderte Änderungs- bzw. Anpassungsbedarf der Geschäftsprozesse sich nicht nur auf die Leistungsprozesse einer Unternehmung beziehen soll, sondern auch auf diejenigen Prozesse und betrieblichen IS gerichtet ist, die der Unterstützung der Leistungsprozesse dienen. ${ }^{335}$ In diesem $\mathrm{Zu}$ -

Vgl. Samtleben/Stadlbauer/Hess (2006), S. 87.

Vgl. Hansen/Neumann (2005), S. 233.

Vgl. Heß (2005), S. 19.

Vgl. Heß (2005), S. 19.

Vgl. Lankes et al. (2008), S. 12.

Vgl. Berbner et al. (2005), S. 268. Nach ANDRESEN/GRONAU resultiert der Anpassungsbedarf der Geschäftsprozesse aus den äußeren Einflüssen, die sprunghaft und dynamisch auftreten können. Vgl. Andresen/Gronau (2005), S. 30.

Vgl. Schelp (2007), S. 143f. 
sammenhang konstatieren MODJO KAMNENG ET AL., dass insbesondere die geringe IT-Unterstützung von Geschäftsprozessen, deren Abläufe nicht im Vorfeld festgelegt sind, ein aktuelles Problemfeld darstellt. ${ }^{336}$

Eine weitere wichtige Triebfeder, die sich aus den Nennungen zu den SOATreibern schlussfolgern lässt, liegt in der Forderung nach einer ganzheitlichen bzw. durchgängigen Unterstützung der Geschäftsprozesse über Systemgrenzen hinweg mit dem Ziel, ${ }^{337}$ auf diese Weise die Wettbewerbsfähigkeit von Unternehmungen sicher zu stellen. ${ }^{338}$ Betroffen sind hiervon nicht nur die unternehmungsinternen, sondern auch die -übergreifenden Prozesse. ${ }^{339}$ Dies impliziert wiederum, dass nicht nur die unternehmungsinternen, sondern auch die externen Anwendungssysteme für eine ganzheitliche Prozessunterstützung zu integrieren sind. Eine Verknüpfung der heterogenen und monolithischen Anwendungssysteme auf der Prozessebene stellt eine wichtige Anforderung dar, damit eine Automatisierung der Geschäftsprozesse, die auf die jeweiligen Systeme aufsetzen, erfolgen kann. ${ }^{340}$

Gefordert wird in diesem Zusammenhang der Betrieb einer flexiblen ITArchitektur, um die Vielzahl der heterogenen Altanwendungen, Plattformen, Betriebssysteme und Kommunikationsmechanismen zu integrieren und eine höhere Adaptierbarkeit der Geschäftsprozesse zu erreichen. ${ }^{341}$ Eine IT-Architektur umfasst neben der IS-Architektur die Vorgehensmodelle, die zur Gestaltung der IS zum Einsatz kommen. ${ }^{342} \mathrm{Zu}$ unterscheiden ist eine IT-Architektur von einer Geschäftsarchitektur, die die Definition und Strukturierung der Geschäftsprozesse, Geschäftsobjekte und Organisationsstrukturen fokussiert. ${ }^{343}$ Nach diesem Verständnis setzen sich die Geschäfts- und die Systemarchitektur zur Unternehmungsarchitektur zusammen. ${ }^{344}$

Mit Blick auf die eingangs beschriebenen vertikal organisierten ITStrukturen einer Unternehmung ist festzustellen, dass einer Unterstützung von Geschäftsprozessen über Systemgrenzen hinweg durch den Einsatz monolithi-

336

Vgl. Modjo Kamneng et al. (2007), S. 289.

Vgl. Heß (2005), S. 19.

Vgl. Grimm (2005), S. 17. Nach GLÖCKLE liegt hierin das Ziel der IT-Integration. Um dieses Ziel zu erreichen, sind dem Autor zufolge eine Infrastruktur sowie die notwendigen Ressourcen bereitzustellen. Vgl. Glöckle (2007), S. 8.

Vgl. Grimm (2005), S. 17.

Vgl. Kossmann/Leymann (2004), S. 117.

Vgl. Keller (2002), S. 25.

Vgl. Ackermann et al. (2006), S. 184.

Vgl. Foegen/Battenfeld (2001), S. 293. Eine alternative Sichtweise, die keine begriffliche Trennung zwischen einer Geschäftsarchitektur und einer IT-Architektur vorsieht, vertreten MüllER ET AL. Nach der Auffassung der Autoren setzt sich eine ITArchitektur aus der verwendeten Hard- und Software, den Geschäftsprozessen und der Organisationsstruktur einer Unternehmung zusammen. Vgl. Müller et al. (2006), S. 187. Vgl. Foegen/Battenfeld (2001), S. 293f. 
scher und heterogener Anwendungssysteme augenblicklich i.d. R. enge Grenzen gesetzt sind. Daraus ergibt sich die Gefahrenquelle, dass die so genannte kunden- sowie partnerorientierte Agilität nicht mehr gewährleistet werden kann. Unter kundenorientierter sowie partnerorientierter Agilität lässt sich die Fähigkeit einer Unternehmung auffassen, von seinen Kunden sowie Geschäftspartnern zu lernen und auf Basis der neu erworbenen Fähigkeiten und Kompetenzen neue Geschäftspotenziale zu identifizieren, die es in einem nächsten Schritt gemeinsam mit den Partnern umzusetzen gilt. Im Fokus der kunden- und partnerorientierten Agilität liegen die Geschäftsprozesse, die im ersten Fall beispielsweise durch den Einsatz von Kundenportalen, im zweiten Fall durch die Gestaltung einer unternehmungsübergreifenden Logistikkette z. B. unter Verwendung eines Supply Chain Management-Tools durch die betriebliche IT unterstützt werden können. ${ }^{345}$

Nachdem in den bisherigen Ausführungen, ausgehend von einer Zustandsbeschreibung der betrieblichen IS, die wesentlichen Treiber vorgestellt wurden, die zu einem wachsenden Interesse an SOA beigetragen haben, widmen sich die folgenden Ausführungen den Eigenschaften sowie den Potenzialen, die im Zusammenhang mit diesem derzeit viel beachteten Ansatz diskutiert werden.

\subsection{Sichtweisen auf SOA}

Der schillernde Begriff „SOA“ hat insbesondere seit 2005 eine Reihe von Vorschlägen hervorgebracht, die sich mit den Fragen beschäftigen, was unter einer SOA zu verstehen ist und welchen Beitrag SOA für die Unterstützung einer integrierten Informationsverarbeitung leisten kann. ${ }^{346}$ Eine z. T. euphorische Erwartungshaltung hinsichtlich der Bedeutung von SOA wird nicht nur von vielen Beratungs-, Marktforschungs- und Softwareunternehmungen vertreten, die in SOA einen Megatrend sehen. ${ }^{347}$ Die positive Einschätzung von SOA kommt auch in vielen wissenschaftlichen Beiträgen zum Ausdruck. ${ }^{348}$ Der Aussage von

Vgl. Schelp/Winter (2007), S. 43.

Die Aktualität des Einsatzes einer SOA lässt sich u. a. durch eine Studie belegen, die im Jahr 2007 von AMADEE/MARTIN durchgeführt wurde. Während $85 \%$ der in dieser Studie befragten Unternehmungen SOA eine Bedeutung einräumen, spielt dieses Thema für $50 \%$ der Unternehmungen eine große oder sehr große Rolle. Vgl. Amadee/Martin (2007), S. 6.

347 Vgl. Buxmann/Hess/Widjaja (2007), S. 1316. Gleichzeitig weisen KACZMAREK/WECEL darauf hin, dass der SOA-Begriff gerade von diesen Unternehmungen übermäßig und in einem falschen Verständnis gebraucht wurde. Vgl. Kaczmarek/Wecel (2008), S. 52.

348 HOLTSCHKE/HEIER/HUMMEL bemerken in diesem Zusammenhang, dass die mediale Aufmerksamkeit des SOA-Begriffs derzeit einen für Innovationen typischen Verlauf nimmt, bei diesem nach anfänglichen Übertreibungen und Fehleinschätzungen eine Phase der Ernüchterung zu erwarten ist. Vgl. Holtschke/Heier/Hummel (2009), S. 120.

Alexander Pastwa - 978-3-631-75488-7 
CONRAD ET AL. zufolge handelt es sich bei einer SOA um die Ausprägung einer prozessorientierten Architektur, die aktuell am meisten diskutiert wird. ${ }^{349}$ Nach der Auffassung von DuECK steht SOA für das Hype-Kürzel des Jahres 2006.350 Während nach der Einschätzung von LAARTZ die betriebswirtschaftlichen Auswirkungen einer SOA als revolutionär einzustufen sind, ${ }^{351}$ favorisieren KARCH ET AL. die Bezeichnung "sanfte Revolution" zur Einordnung der Potenziale einer SOA. ${ }^{352}$

Die zu erwartenden Auswirkungen von SOA auf die integrierte Informationsverarbeitung werden ebenfalls in der Aussage von HANSEN/NEUMANN deutlich, die davon sprechen, dass SOA das kommende Jahrzehnt von Integrationslösungen beherrschen wird. ${ }^{353}$ Für VOM BROCKE steht SOA gar für eine neue Generation von Anwendungssystemen. ${ }^{354} \mathrm{Zu}$ einem positiven Urteil kommt auch KRUCZYNSKI, für den kein Zweifel besteht, dass SOA die Informationstechnologie hin zu mehr Flexibilität und Performancegewinn begünstigen wird. ${ }^{355}$ MELzer ET AL. räumen SOA gar das Potenzial ein, sich nach der objektorientierten Programmierung als das nächste Paradigma in der Informatik zu etablieren. ${ }^{356}$ Damit repräsentiert die letztgenannte Einschätzung, bei SOA handele es sich um ein neues Paradigma bzw. einen Paradigmenwechsel, den augenblicklich populärsten Versuch, die Potenziale bzw. Auswirkungen von SOA auf wenige Schlagworte zu reduzieren. ${ }^{357}$

Bisher ist es keinem Beitrag gelungen, ein Begriffsverständnis oder ein klar umrissenes Begriffsgebäude zu SOA zu präsentieren, das zu einer autorenübergreifenden Zustimmung geführt hat. ${ }^{358}$ Einerseits sind die in der Literatur prä-

Vgl. Conrad et al. (2006), S. 88.

Vgl. Dueck (2006), S. 366.

Vgl. Laartz (2008), S. 72.

Vgl. Karch et al. (2004), S. 209.

Vgl. Hansen/Neumann (2005), S. 532.

Vgl. vom Brocke (2007), S. 84.

Vgl. Kruczynski (2006), S. 24.

Vgl. Melzer et al. (2007), S. vii.

Vertreter dieser Sichtweise sind beispielsweise HÖß ET AL., HofMANN, KÜSTER, SIEBENHAAR ET AL. und SCHRÖPFER/SCHÖNHERR. Vgl. Höß et al. (2007), S. 39, 40 und 45; Hofmann (2003), S. 27; Küster (2003), S. 5; Siebenhaar et al. (2008), S. 325; Schröpfer/Schönherr (2008), S. 409.

Vgl. Buxmann/Hess/Widjaja (2007), S. 1316; Höß et al. (2007), S. 40; Liebhart (2007), S. 6. KACZMAREK/WECEL bemerken in diesem Zusammenhang die Existenz hunderter Definitionen zum SOA-Konzept. Vgl. Kaczmarek/Wecel (2008), S. 52. Die Feststellung, dass das derzeit vorherrschende Begriffsverständnis zu SOA durch Heterogenität gekennzeichnet ist, wird ferner durch die Initiative der Bundesregierung untermauert, die im Rahmen der CEBIT 2007 gemeinsam mit dem Branchenverband BITKOM Anforderungen an eine SOA-Taskforce formuliert hat, deren Aufgabenbereich u. a. die Definition grundlegender Begriffe umfasst. Vgl. Buxmann/Hess/Widjaja (2007), S. 1316. 
sentierten Definitionsansätze von Überschneidungen, andererseits von Unterschieden gekennzeichnet. ${ }^{359}$ Auch im Hinblick auf die Vielfalt der in den Beiträgen diskutierten Merkmale einer SOA ist zu konstatieren, dass sowohl allgemeine als auch sehr fokussierte Definitionsvorschläge zu einem uneinheitlichen Verständnis beigetragen haben. ${ }^{360}$ Während in einigen Beiträgen technische Aspekte im Vordergrund stehen, favorisieren andere die fachlichen bzw. betriebswirtschaftlichen Nutzungspotenziale einer SOA. ${ }^{361}$ Zudem zeichnen sich die Beiträge in erschwerender Weise durch eine unterschiedliche Gewichtung der in den jeweiligen Ansätzen thematisierten Eigenschaften einer SOA aus. ${ }^{362}$ Auch wenn die aktuell existierenden SOA-Auslegungen keine scharfe Begriffserklärung liefern, ${ }^{363}$ lassen sich dennoch einige Merkmale einer SOA anführen, die zumindest von einer Mehrheit der Autorenschaft thematisiert werden und daher als SOA-konstituierende Eigenschaften bezeichnet werden können.

Um die Spannweite der verschiedenen SOA-Begriffsdefinitionen zu verdeutlichen und darauf aufbauend in Abschnitt 3.5 ein integriertes Begriffsverständnis zu präsentieren, werden zunächst exemplarisch einige Definitionen vorgestellt. Aus den Literaturvorschlägen zur Bestimmung des SOA-Begriffs lassen sich drei Strömungen identifizieren, die in den folgenden Abschnitten konkretisiert werden. Ausgehend von einem konzeptionell geprägten Begriffsverständnis, das in Abschnitt 3.2.1 präsentiert wird, widmet sich Abschnitt 3.2.2 einer Perspektive, in der die technische Implementierung einer SOA im Vordergrund steht. Alternativ zu den beiden vorangehenden Sichtweisen fokussiert Abschnitt 3.2.3 ein Begriffsverständnis, das die gestalterischen Aspekte einer SOA betont.

\subsubsection{SOA als Informationssystem-Architektur}

Der ersten Auslegung des SOA-Begriffs liegt die Vorstellung zugrunde, dass sich SOA als Informationssystem-Architektur (IS-Architektur) auffassen lässt. ${ }^{364}$ Als Vertreter dieser Sichtweise wird der Definitionsansatz von MELZER ET AL. vorgestellt. Die Autoren bemerken, dass es sich bei einer SOA um eine Systemarchitektur handelt, „die vielfältige, verschiedene und eventuell inkompatible Methoden oder Applikationen als wiederverwendbare und offen zu-

Vgl. Melzer et al. (2007), S. 11.

360 Vgl. hierzu auch Melzer et al. (2007), S. 11. Auf die unterschiedliche Schwerpunktsetzung im Themenfeld SOA verweisen auch de Hesselle/Klüpfel/Bauersachs (2008), S. 30 .

361 Vgl. Buxmann/Hess/Widjaja (2007), S. 1316; Kaczmarek/Wecel (2008), S. 52; Schröpfer/Schönherr (2008), S. 409.

362 Vgl. hierzu auch Richter/Haller/Schrey (2005), S. 413.

$363 \mathrm{Vgl}$. de Hesselle/Klüpfel/Bauersachs (2008), S. 30.

$364 \mathrm{Zu}$ dieser Überzeugung kommt auch Coldewey (2007), S. 50. 
greifbare Dienste repräsentiert und dadurch eine plattform- und sprachunabhängige Nutzung und Wiederverwendung ermöglicht". ${ }^{365}$ Wie aus diesem Definitionsvorschlag hervorgeht, sind sowohl der Architekturgedanke als auch die Serviceorientierung zentrale Charakteristika einer SOA. Mit Blick auf den Aspekt der Serviceorientierung liegt der Leitgedanke einer SOA darin, Methoden, die eine fachliche Logik repräsentieren, softwaretechnisch in Form von Diensten bzw. Services abzubilden und für eine mehrfache Nutzung zur Verfügung zu stellen. ${ }^{366}$ Eine hohe Wiederverwendbarkeit hat zur Folge, dass sich die implementierten Services für viele Problemstellungen bzw. in einer Vielzahl von Anwendungen nutzen lassen. ${ }^{367}$ Die Dienste lassen sich dabei aus den Realisierungen der diversen betrieblichen Anwendungen gewinnen. ${ }^{368}$ Als technisches sowie konzeptionelles Fundament dient eine spezielle IS-Architektur, die die Autoren als SOA bezeichnen. ${ }^{369}$

Mit ihrer SOA-Auslegung folgen MELzER ET AL. der gebräuchlichen Praxis, den Architekturbegriff für Konzepte der Informationsverarbeitung zu verwenden. ${ }^{370}$ Ein Zugang zum Architekturbegriff lässt sich über ein allgemeines Begriffsverständnis erzielen. ${ }^{371}$ Dieses besagt, dass eine Architektur die Struktur eines Systems definiert, ${ }^{372}$ die sich ganzheitlich sowie auf einer abstrakten Ebene betrachten lässt. ${ }^{373}$ Eine Architektur beschreibt sowohl die logische und physikalische Anordnung der Bausteine eines komplexen Systems als auch ihre Beziehungen zueinander, ${ }^{374}$ wobei im Mittelpunkt die Darstellung der funktionellen Zusammenhänge eines Systems steht. ${ }^{375}$ Darüber hinaus sind innerhalb einer Architektur Richtlinien vorgegeben, die dazu dienen, die Systemkomponenten sowie ihre Beziehungen zueinander zu gestalten und weiterzuentwickeln. ${ }^{376}$

Eine IS-Architektur steht für die Idee, eine strukturelle Sichtweise mit einer modellorientierten Perspektive zu verbinden. ${ }^{377} \mathrm{Da}$ die Beschreibung auf einer grobgranularen Ebene stattfindet, steht das Grobdesign des IS im Mittel-

Melzer et al. (2007), S. 11.

FIEGE/STELZER zufolge ist gerade die Wiederverwendbarkeit der Services eines der wichtigsten Architekturziele einer SOA. Vgl. Fiege/Stelzer (2007), S. 911.

Vgl. Winkler (2007), S. 258.

Vgl. Gimnich (2007), S. 68.

Als alternative Bezeichnung lässt sich auch der Begriff Systemarchitektur verwenden.

Vgl. Strunz (1990), S. 441.

HERDEN ET AL. weisen in diesem Zusammenhang darauf hin, dass sich bisher kein einheitliches Verständnis zum Architekturbegriff durchgesetzt hat. Vgl. Herden et al. (2006), S. 1.

Vgl. Petersohn (2004), S. 16.

Vgl. Aier/Schönherr (2006), S. 188.

Vgl. Hansen/Neumann (2005), S. 174; Bleek/Wolf (2008), S. 84.

Vgl. Petersohn (2005), S. 21.

Vgl. Heutschi/Legner/Österle (2006), S. 362.

Vgl. Krcmar (2003), S. 39. 
punkt. ${ }^{378}$ Neben der softwaretechnischen Architektur ist die Definition einer Infrastrukturarchitektur als weiterer Teilbereich einer Systemarchitektur von Bedeutung. ${ }^{379}$ Charakteristisch für eine softwaretechnische Architektur bzw. Softwarearchitektur ist, dass ihre Softwarekomponenten, ihre Schnittstellen und ihre Beziehungen beschrieben werden. ${ }^{380}$ Die Komponenten lassen sich dabei auf unterschiedlichen Abstraktionsebenen betrachten, wobei jede Abstraktionsebene die darunter liegenden Details verbirgt. ${ }^{381}$ Im Vergleich zur softwaretechnischen Architektur zeichnet sich die Infrastrukturarchitektur durch eine ganzheitliche, operationale Perspektive auf das zu gestaltende System aus. Der Fokus bei der Infrastrukturarchitektur richtet sich zum einen auf das technische System, d. h. auf die Hardware, Plattformen und deren Verbindungen, zum anderen auf die Platzierung der Softwarekomponenten innerhalb des Systems. Darüber hinaus stehen bei der Infrastrukturarchitektur die Konfiguration und das Management des Systems im Mittelpunkt. ${ }^{382}$

Einen ergänzenden Aspekt zur Sichtweise von MELZER ET AL. fügen HEUTSCHI/LEGNER/ÖSTERLE an, nach deren Auffassung es sich bei einer SOA um eine ,mehrschichtige, verteilte Informationssystem-Architektur (IS)Architektur handelt, die Teile der Applikationsarchitektur als Services kapselt und dabei eine Reihe von Designprinzipien berücksichtigt" “. ${ }^{383}$ In Analogie zum Definitionsansatz von MELZER ET AL. betonen die Autoren die Bedeutung von Diensten, die auf der fachlichen Logik der Anwendungssysteme basieren und nach bestimmten Vorgaben in Form von Designprinzipien zu entwickeln und zu nutzen sind. Eine Applikationsarchitektur richtet sich auf die Beschreibung der statischen und dynamischen Strukturen von Anwendungssystemen. ${ }^{384}$ Mit Blick auf den Architekturaspekt einer SOA verweisen HEUTSCHI/LEGNER/ÖSTERLE in Erweiterung des Begriffsverständnisses von MELZER ET AL. darauf, dass es sich bei einer SOA nicht um ein zentralisiertes, monolithisches Konstrukt, sondern um eine verteilte IS-Systemarchitektur handelt.

Die Eigenschaft einer verteilten Architektur bedeutet, dass sich die Hardware- und Softwarekomponenten, die im Rahmen einer SOA zum Einsatz kommen, auf vernetzten Computerressourcen befinden, wobei eine Kommunikation zwischen diesen Komponenten über den Austausch von Nachrichten erfolgt. 385 Der Aspekt der Verteilung bezieht sich in diesem Zusammenhang auf

Vgl. Hansen/Neumann (2005), S. 223.

Vgl. Foegen/Battenfeld (2001), S. 294ff.

Vgl. Fink/Schneidereit/Voß (2005), S. 211.

Vgl. Foegen/Battenfeld (2001), S. 296.

Beim letzten Aspekt sind beispielsweise die Kapazitätsplanung, die Datensicherung und der Wiederanlauf von Bedeutung. Vgl. Foegen/Battenfeld (2001), S. 294.

Heutschi/Legner/Österle (2006), S. 362.

Vgl. Müller (2007b), S. 143.

Siehe hierzu die Definition eines verteilten Systems bei Hammerschall (2005), S. $16 \mathrm{f}$. 
die Möglichkeit, in einer SOA bestehende Applikationen durch die Vernetzung autonomer und plattformunabhängiger Dienste zu kollaborativen Anwendungen weiter zu entwickeln. ${ }^{386}$

Die bisherigen Definitionsansätze werden von RICHTER/HALLER/SCHREY und HESS/Humm/VOß um einen weiteren Aspekt ergänzt. Den Autoren zufolge handelt es sich bei einer SOA um ,ein Architekturmuster, das den Aufbau einer Anwendungslandschaft aus einzelnen fachlichen Anwendungsbausteinen beschreibt, die jeweils eine klar umrissene Aufgabe wahrnehmen. Die Anwendungsbausteine sind lose miteinander gekoppelt, indem sie einander ihre Funktionalitäten in Form von Services anbieten" ${ }^{387}$ Mit Blick auf den Architekturmusterbegriff wird in der Definition von RICHTER/HALLER/SCHREY deutlich, dass SOA als Referenzarchitektur verstanden wird, die für die Implementierung sowie Nutzung verschiedener Anwendungsbausteine in Form von Services zum Einsatz kommen kann. ${ }^{388}$

Ganz besonders ist es den Autoren wichtig, auf das Prinzip der losen Kopplung hinzuweisen. Dieses liefert zum einen die Voraussetzung für die Inanspruchnahme der Funktionalitäten der Dienste, die für einen bestimmten Einsatzzweck konzipiert und implementiert werden. ${ }^{389}$ Zum anderen soll die Einhaltung dieses Prinzips bewirken, dass die Schwachstellen, die sich infolge des Einsatzes monolithischer Systeme ergeben, ${ }^{390}$ durch die einfache Kopplung und Entkopplung einzelner Funktionen sowie kompletter Anwendungssysteme auf Basis entsprechender Services beseitigt werden. ${ }^{391}$

\subsubsection{SOA als konkrete technische Implementierung eines Softwaresystems auf Basis von Web Services}

Während in den bisherigen Definitionsansätzen SOA auf einer abstrakten Ebene betrachtet wurde, fokussiert der Vorschlag von LAUDON/LAUDON/SCHODER die konkrete, technische Implementierung einer SOA. Ihre Definition lautet: „A collection of Web services that are used to build a firm's software systems constitutes what is known as a service-oriented architecture. A service-oriented architecture (SOA) is a set of self-contained services that communicate with each

Vgl. Eymann/Winter (2008), S. 70.

Richter/Haller/Schrey (2005), S. 413.

Vor diesem Hintergrund greift die Definition von FoEgEN/BATTENFELD, die ein Architekturmuster mit einer Referenzarchitektur gleichsetzen, die sowohl funktionale als auch operationale Aspekte für eine bestimmte Klasse von Anwendungen beschreibt. Vgl. Foegen/Battenfeld (2001), S. 298.

Vgl. hierzu Abschnitt 3.3.2.2.

Vgl. Abschnitt 3.1.2.

Siehe hierzu Abschnitt 3.1.3.1, der die Kosten des IT-Betriebs als Treiber für das Interesse an SOA thematisiert. 
other to create a working software application."392 Weitere Vertreter für die technikorientierte Sichtweise sind TERZIDIS/SURE/BRELAGE. Ihre Definition hat folgenden Wortlaut: „SOA modularizes monolithic software packages into manageable application components which are offered as standardized Web Services in an expandable repository". 393

Im Unterschied zu den bereits präsentierten Begriffsverständnissen wird in diesen beiden Definitionsvorschlägen deutlich, dass für die Autoren die Realisierung einer SOA gleichbedeutend mit dem Einsatz von Web Services ist. ${ }^{394}$ Folglich lässt sich dieser Definitionsvorschlag als ein sehr enges, technikorientiertes Begriffsverständnis einer SOA werten.

\subsubsection{SOA als Konzept zur Gestaltung prozessorientierter Anwendungen}

Neben dem konzeptionell geprägten sowie technikorientierten Begriffsverständnis betont eine andere Sichtweise das gestalterische Potenzial, das der SOA-Ansatz bietet. Einen entsprechenden Definitionsansatz liefert die Marktforschungsunternehmung FORRESTER RESEARCH. Diesem Vorschlag zufolge handelt es sich bei einer SOA um die „Art und Weise, wie Anwendungen und SW-Architekturen gestaltet, bereitgestellt und verwaltet werden". ${ }^{395}$ In dieser Definition wird die bisher statische Perspektive auf eine SOA um eine dynamische Sichtweise ergänzt. In Abgrenzung zu den vorangehenden Definitionen steht nach der Vorstellung von FORRESTER RESEARCH der Begriff einer SOA für einen bestimmten Stil, wie Anwendungen und SW-Architekturen entwickelt und betrieben werden können.

Den Fokus auf den Gestaltungsaspekt einer SOA legen auch LOMOW/ NEWCOMER. Ihr Definitionsansatz lautet: „A service-oriented architecture is a style of design that guides all aspects of creating and using business services throughout their lifecycle (from conception to retirement). An SOA is also a way to define and provision an IT infrastructure to allow different applications to exchange data and participate in business processes, regardless of the operating systems or programming languages underlying those applications." 396 Ein wichtiger Akzent in dieser Definition richtet sich auf den Prozess zur Entwicklung der Dienste sowie den gesamten Lebenszyklus der im Rahmen einer SOA zu nutzenden Services. Nach NEwCOMER/LOMOW steht der Ansatz einer SOA

392 Laudon/Laudon/Schoder (2006), S. 214.

393 Terzidis/Sure/Brelage (2008), S. 76.

394 Neben Laudon/Laudon/Schoder und Terzidis/Sure/Brelage gelten als weitere Vertreter dieser Sichtweise beispielsweise ERL, LIEBHART, RIEKS und STREIBICH. Vgl. Erl (2005), S. 54; Liebhart (2007), S. 5 und 11; Rieks (2006), S. 7; Streibich (2008), S. 73. Zur Technologie der Web Services vgl. Abschnitt 3.4.

396 Newcomer/Lomow (2005), S. 13. 
für einen Entwurfsstil, der sowohl der Entwicklung als auch der Nutzung der Services zugrunde liegt. Gleichzeitig verstehen die Autoren SOA als Gestaltungsprozess zur Entwicklung und zum Betrieb einer IT-Infrastruktur, die dazu dient, losgelöst von der softwaretechnischen Ausprägung der zum Einsatz kommenden Anwendungssysteme, eine Unterstützung der Geschäftsprozesse zu realisieren.

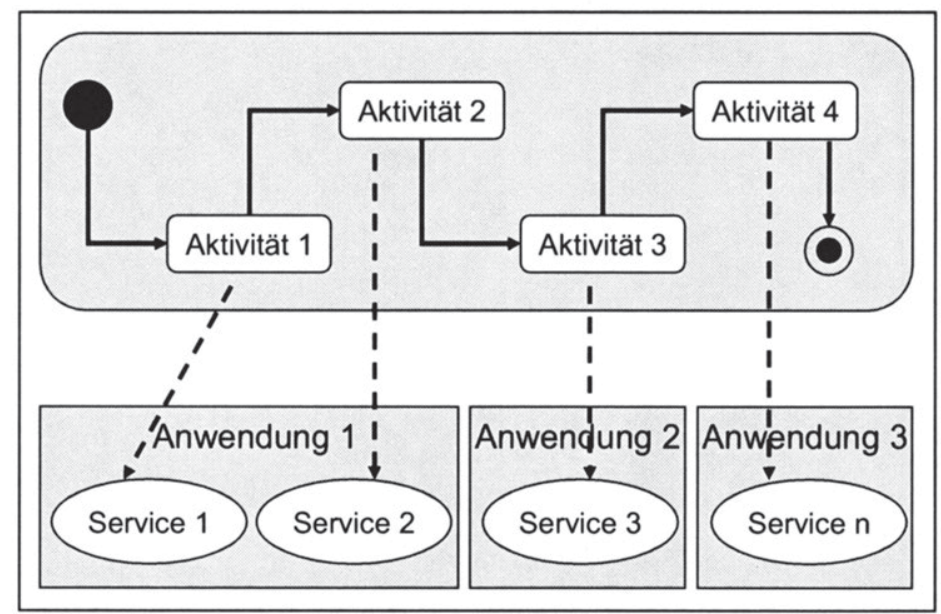

Abbildung 14: Zusammenhang zwischen Prozessaktivitäten, Services und Anwendungssystemen

Hess/Humm/Voß ergänzen, dass der Aufruf von Services über einen einheitlichen Mechanismus es erlaubt, „Geschäftsprozesse auf der Basis von Services möglichst einfach zu implementieren und zu ändern“. ${ }^{397}$ Das Anwendungsfeld der in einer SOA eingebundenen Dienste richtet sich dabei nach der Auffassung von HESS/HUMM/VOß auf die Unterstützung von Geschäftsprozessen, die durch die Verknüpfung voneinander unabhängiger Services, an Flexibilität gewinnen können. Diese Aussage wird durch Abbildung 14 gestützt. Wie in dieser Abbildung zu erkennen ist, werden die Dienste, die innerhalb einer Anwendung eine Aufgabe erfüllen, zu einer Prozesskette verknüpft und als Prozessaktivitäten 
ausgeführt. Das Zusammenfügen von Services wird auch als Komposition bezeichnet.

Im Ergebnis verdeutlicht die Definition von HESs/HuMM/VOB, dass SOA nicht nur einen rein technischen Bezug, sondern auch einen fachlichen Anspruch hat, der sich sowohl auf die Automatisierung als auch auf die Flexibilisierung von Geschäftsprozessen richtet.

\subsection{Services als Basiselemente einer SOA}

Losgelöst von ihren unterschiedlichen Ausrichtungen, zeichnen sich die in $\mathrm{Ab}$ schnitt 3.2 präsentierten Auslegungen einer SOA durch die Gemeinsamkeit aus, dass die Softwareservices das zentrale Charakteristikum einer SOA darstellen. ${ }^{398}$ Die bisherigen, in der SOA-Literatur erarbeiteten Vorschläge zur Definition des Servicebegriffs haben zu einem Erkenntnisstand geführt, bei dem verschiedene Sichtweisen miteinander konkurrieren. In diesem Zusammenhang ist zu konstatieren, dass es sich bei den verschiedenen Ansätzen zur Beschreibung und Abgrenzung eines Services bzw. eines Dienstes nicht um disjunkte Definitionsvorschläge handelt. 399 Vielmehr beschränken sich die Autoren in ihren Beiträgen auf die Darstellung verschiedener, z. T. sich ergänzender Aspekte, die nicht im Gegensatz zu den Inhalten anderer Bestimmungsversuche stehen.

Das Ziel der folgenden Ausführungen liegt darin, die in den verschiedenen Literaturbeiträgen präsentierten Eigenschaften und Gestaltungsprinzipien von Services zu einem vollständigen Begriffsverständnis zusammenzuführen. Mit Blick auf ihre Bedeutung als fundamentale Bausteine einer SOA widmen sich die Ausführungen des Abschnitts 3.3.1 der Frage, welche charakteristischen Eigenschaften Services aufweisen, die innerhalb einer SOA genutzt werden. ${ }^{400}$ Nach den Grundlagen zu den Bestandteilen eines Services werden in Abschnitt 3.3.2 zentrale Gestaltungsprinzipien vorgestellt, die bei der Entwicklung sowie dem Einsatz von Services zu berücksichtigen sind.

398 Dass Services das zentrale Designobjekt einer SOA darstellen, wird auch bei HANHART/ LEGNER/ÖSTERLE deutlich. Vgl. Hanhart/Legner/Österle (2008), S. 478.

399 Zur Vermeidung einer sprachlichen Monotonie werden die Begriffe „Services“ und „Dienste“ im Folgenden synonym verwendet.

400 Zur Bedeutung der Services im Rahmen einer SOA vgl. Newcomer/Lomow (2005), S. 55. Dass Services das wichtigste Element einer SOA repräsentieren, wird auch bei LIEBHART deutlich. Vgl. Liebhart (2007), S. 8. 


\subsubsection{Begriff des Services}

Aus einer softwaretechnischen Perspektive betrachtet, 401 lassen sich Services als Softwarekomponenten verstehen, die eine abgegrenzte und standardisierte Funktionalität anbieten, die wiederum Bestandteil eines größeren Verarbeitungsablaufs ist. ${ }^{402}$ Services werden über eine Schnittstelle lokal oder über ein Netzwerk aufgerufen. Dieser Vorstellung folgend, weisen Dienste den Charakter von abgegrenzten Softwaremodulen auf, die als Programmbausteine sowohl in Form von Prozeduren als auch Funktionen softwaretechnisch implementiert werden können. ${ }^{403}$ Die Bestandteile eines Services sowie die zwischen diesen existierenden Beziehungen lassen sich anhand eines Servicemodells verdeutlichen, das in Abbildung 15 dargestellt ist.

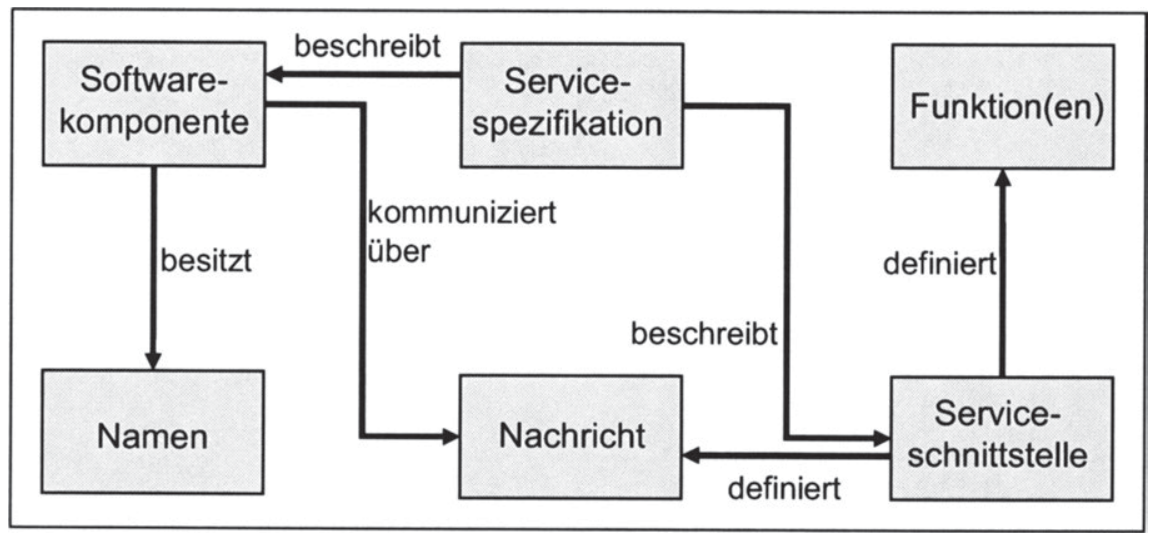

Abbildung 15: Servicemodell

Ein Service stellt eine Softwarekomponente dar, die einen Namen besitzt und über diesen Namen aufgerufen werden kann. Darüber hinaus verfügen Dienste über eine Schnittstelle, die den Zugriffspunkt auf die von dem jeweiligen Service bereitgestellte Funktionalität darstellt. ${ }^{404}$ Die Funktionalität steht für eine klar umrissene Aufgabe, die der Service erbringen soll. Zur Bereitstellung der Funktionalität werden ein oder mehrere Serviceoperationen ausgeführt, die in der Serviceschnittstelle definiert werden.

401 EYMANN/WINTER verdeutlichen, inwieweit sich der softwaretechnisch geprägte Servicebegriff, der einer SOA zugrunde liegt, aus dem Serviceverständnis ableiten lässt, das in der Wirtschaftswissenschaft vorherrscht. Vgl. Eymann/Winter (2008), S. 70. Zum betriebswirtschaftlich orientierten Servicebegriff vgl. Buhl et al. (2008), S. 61 f.

Vgl. Humm/Juwig (2006), S. 2.

403 Vgl. Richter/Haller/Schrey (2005), S. 413.

404 Vgl. Liebhart (2007), S. 9; Samtleben/Stadlbauer/Hess (2006), S. 91. 
Der Aufruf von Diensten bzw. die Kommunikation von Diensten untereinander erfolgt über den Austausch von Nachrichten. In diesem Zusammenhang besitzt die Serviceschnittstelle die Aufgabe, die für die Inanspruchnahme eines Dienstes notwendigen Eingabe- und Ausgabeparameter sowie die Struktur der Nachricht festzulegen. 405 Als weiterer Bestandteil eines Dienstes ist die Servicespezifikation, die auch als Servicebeschreibung oder Servicevertrag bezeichnet wird, zu nennen. Die Servicebeschreibung führt alle Informationen zur Servicenutzung auf, die über die Schnittstellenbeschreibung hinausgehen. Während die Servicespezifikation einen Dienst beschreibt, handelt es sich bei der Serviceschnittstelle um die softwaretechnische Realisierung einer Servicespezifikation. 406

\subsubsection{Prinzipien zum Design von Services}

Ausgehend von dem im vorherigen Abschnitt präsentierten, einer SOA zugrunde liegenden Verständnis zum Dienstbegriff, widmen sich die Ausführungen dieses Abschnitts der Frage, welche grundsätzlichen Empfehlungen für den Entwurf einer SOA zu berücksichtigen sind. ${ }^{407}$ Hierzu wurde in den relevanten Beiträgen der vergangenen Jahre eine Reihe von Prinzipien vorgeschlagen, die sich vor diesem Hintergrund auch als Designprinzipien bezeichnen lassen. ${ }^{408}$ In Analogie zum Ansatz der Objektorientierung, in dessen Rahmen beispielsweise die Prinzipien des Information Hiding (Geheimnisprinzip), der Vererbung, des Polymorphismus, usw. charakteristische Konzepte bilden, lassen sich auch im Zusammenhang mit dem Ansatz der Serviceorientierung allgemeingültige Prinzipien ableiten. ${ }^{409}$

Eine von HEUTSCHI/LEGNER/ÖSTERLE durchgeführte Auswertung ausgewählter Beiträge hat ergeben, dass sich bisher kein Konsens herauskristallisiert

405 Vgl. Heutschi/Legner/Österle (2006), S. $363 \mathrm{f}$.

406 Vgl. Heutschi (2007), S. 191.

407 Mit Blick auf die Bedeutung des Prinzipbegriffs folgt die vorliegende Arbeit dem Verständnis von BALZERT, nach dessen Auffassung es sich bei Prinzipien um Grundsätze handelt, die sich durch einen allgemeingültigen sowie abstrakten Charakter auszeichnen und sich sowohl aus der Erfahrung und Erkenntnis herleiten als auch durch diese bestätigen oder widerlegen lassen. Vgl. Balzert (2001), S. 36.

408 ERL zufolge ist die Einhaltung der Prinzipien erforderlich, um die Architekturziele einer SOA zu erfüllen. Vgl. Erl (2005), S. 290. Da die von ERL thematisierten Designprinzipien sich allesamt auf den Entwurf von Services beziehen, wird im Folgenden die Auffassung vertreten, dass es sich hierbei um Designprinzipien handelt.

409 Eine Vertiefung der objektorientierten Konzepte liefert beispielsweise BALZERT. Vgl. Balzert (2001), S. 152ff. Zur Vermeidung einer sprachlichen Monotonie werden im Folgenden die Begriffe Prinzipien und Konzepte synonym verwendet. Zum Zusammenhang zwischen den Prinzipien bzw. Konzepten der Serviceorientierung und der Objektorientierung nimmt z. B. ERL Stellung. Vgl. Erl (2005), S. $321 \mathrm{ff}$. 
hat, welche Prinzipien als charakteristisch für eine SOA anzusehen sind. ${ }^{410} \mathrm{Wie}$ die Autoren bemängeln, erweist sich eine Analyse der in den Beiträgen thematisierten Designprinzipien als schwierig, da nicht nur verschiedene Designprinzipien thematisiert werden, sondern auch inhaltlich identische Prinzipien unterschiedlich benannt werden. ${ }^{411}$ Die nachfolgenden Ausführungen zu den Designprinzipien einer SOA orientieren sich an den Ergebnissen der Literaturauswertung von HEUTSCHI/LEGNER/ÖSTERLE. Die in den untersuchten Beiträgen von den Autoren identifizierten Prinzipien werden zu drei übergeordneten Gruppen zusammengefasst, deren Vorstellung Gegenstand der Abschnitte 3.3.2.1 bis 3.3.2.3 ist.

\subsubsection{Prinzip der „Abstraktion von der Implementierungslogik“}

Das Gestaltungsprinzip der „Abstraktion von der Implementierungslogik“ verweist auf das im Rahmen einer SOA angestrebte Ziel, Dienste nutzen zu können, ohne Kenntnisse hinsichtlich ihrer Implementierung besitzen zu müssen. In Analogie zu dem aus der Objektorientierung bekannten Prinzip des Information Hiding 412 wird von den bereitgestellten Services gefordert, Details der Serviceimplementierung wie beispielsweise die internen Datenstrukturen geheim zu halten. 413 Das Verhalten eines Services lässt sich folglich als Black Box charakterisieren, da die sich hinter dem Service verbergenden Implementierungsdetails für den Nutzer eines Dienstes unsichtbar bleiben und i. d. R. auch irrelevant sind. 414

Das Designprinzip der „Abstraktion von der Implementierungslogik“ hat zur Folge, dass alle für die Nutzung eines Dienstes notwendigen Informationen in der Servicespezifikation enthalten sein müssen, 415 die von der Serviceimplementierung getrennt ist. ${ }^{416}$ Diesen Zusammenhang visualisiert Abbildung 16. Hierbei wird deutlich, dass der aufrufende Service A über die Schnittstelle des

410 Vgl. Heutschi/Legner/Österle (2006), S. 364f.

411 Vgl. Heutschi/Legner/Österle (2006), S. 364.

412 Zum Prinzip des Information Hiding vgl. z. B. Balzert (2001), S. 156; Fink/ Schneidereit/Voß (2005), S. 130; Pomberger/Pree (2004), S. 135.

413 Vgl. Heutschi/Legner/Österle (2006), S. 366.

414 Vgl. Erl (2005), S. 298; Melzer et al. (2007), S. 13.

415 In der Auswertung von HeUTSCHi/LEGNER/ÖsterLE wird die Notwendigkeit zur Spezifizierung von Services als eigenes Designprinzip aufgenommen. Da die Servicespezifizierung einen Teilaspekt der Serviceabstraktion darstellt, wird das Designprinzip der Servicespezifizierung im Rahmen der Serviceabstraktion in Abschnitt 3.3.2.1 thematisiert.

416 Auf die Notwendigkeit, die Servicebeschreibung von der Serviceimplementierung zu separieren, verweisen beispielsweise Newcomer/Lomow (2005), S. 8f. 
Dienstes B, die im Rahmen der Servicespezifikation beschrieben ist, dessen Funktionalität in Anspruch nehmen kann.

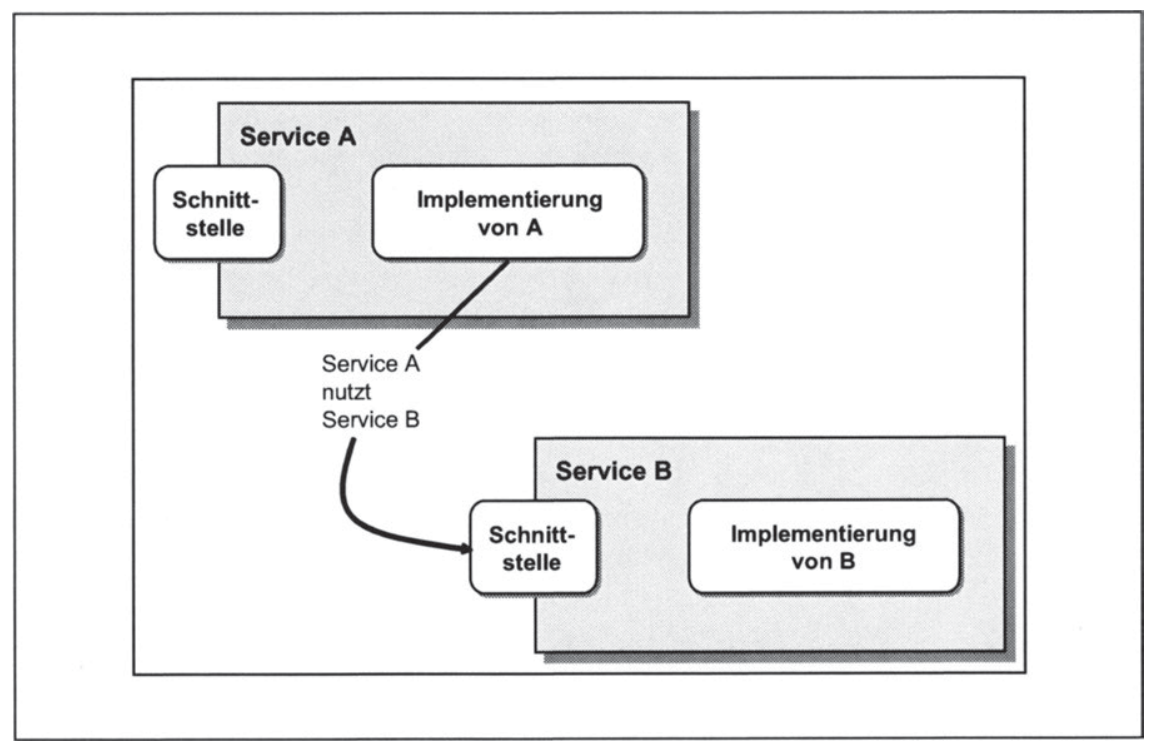

Abbildung 16: Aufruf eines Services 417

\subsubsection{Prinzipien der „hohen Servicekohäsion“ und der „schwachen lo- gischen Kopplung“6}

Mit den Prinzipien der ,hohen Servicekohäsion“ und der „schwachen logischen Kopplung" liegen zwei weitere Designprinzipien vor, die von HEUTSCHI/ LEGNER/ÖsTERLE im Rahmen ihrer Literaturauswertung genannt wurden. In einem allgemeinen Verständnis setzt sich Kopplung (coupling) mit den Wechselbeziehungen zwischen Softwarekomponenten auseinander, während die Kohäsion (cohesion) ein $\mathrm{Ma} ß$ für die Abhängigkeit zwischen den Elementen innerhalb einer Softwarekomponente darstellt. ${ }^{418}$ Als eine Softwarekomponente wird

417 In Anlehnung an Tilkov/Starke (2007), S. 20.

418 Vgl. Hansen/Neumann (2005), S. 225. Siehe hierzu auch die Beiträge von FINK/ SCHNEIDEREIT/VOB und LIEBHART, die in diesem Zusammenhang anstelle von Softwarekomponenten den Modulbegriff verwenden. Vgl. Fink/Schneidereit/Voß (2005), S. 211; Liebhart (2007), S. 162. 
dabei ein Stück Software bezeichnet, das über eine wohldefinierte Schnittstelle klar abgegrenzte Funktionen bereitstellt. 419

Aus der Perspektive der Serviceorientierung steht der Begriff der Servicekohäsion für einen Indikator, der eine Aussage darüber zulässt, inwieweit die von einem Service gekapselten Funktionen und Daten dazu beitragen, einen im Vorfeld definierten Zweck zu erfüllen. ${ }^{420}$ Das Prinzip der „hohen Servicekohäsion“ schreibt in diesem Zusammenhang vor, dass ein Dienst möglichst nur diejenigen Funktionen und Daten kontrolliert, die zur Erfüllung einer logisch abgegrenzten und eigenständigen Aufgabe erforderlich sind. ${ }^{421}$

Im Vergleich zum Prinzip der ,hohen Servicekohäsion“ zielt das Prinzip der „schwachen logischen Kopplung“, das alternativ als Prinzip der losen Kopplung (loose coupling) bezeichnet werden kann, auf das Außenverhältnis der Softwaredienste ab.422 In einer SOA steht der Begriff der Kopplung für die Stärke der Verbindung zwischen den zum Einsatz kommenden Diensten. Der Gegenstandsbereich richtet sich folglich auf den Grad der Abhängigkeit zwischen Serviceangebot und -nachfrage. Für die Entwicklung von Services besitzt das Prinzip der losen Kopplung insoweit eine Relevanz, als der Entwurf, die Implementierung, die Architektur und die zum Einsatz kommende Technologie zur Entwicklung von Services von diesem Designprinzip betroffen sind. ${ }^{423}$

Um einen erfolgreichen Betrieb einer SOA zu gewährleisten, wird angestrebt, eine möglichst schwache logische Kopplung zwischen den Services zu realisieren. D. h., die Kommunikation zwischen einem Service und seinen Nutzern zeichnet sich durch ein hohes Maß an Unabhängigkeit aus. ${ }^{424}$ Durch das Prinzip der losen Kopplung lassen sich mögliche Abhängigkeiten minimieren, die sich aufgrund einer räumlichen Verteilung zwischen dem Ort des Serviceangebots sowie dem Ort der Serviceinanspruchnahme ergeben können. Gleichzeitig stellt dieses Prinzip sicher, dass sich Services ungeachtet der Existenz einer im Vorfeld feststehenden Gruppe von Servicenehmern softwaretechnisch umsetzen und bereitstellen lassen.

Trotz seiner mehrfachen Nennung in den von HEUTSCHI/LEGNER/ÖSTERLE ausgewerteten Literaturbeiträgen besteht hinsichtlich der Relevanz des Prinzips der losen Kopplung als wichtiges Charakteristikum einer SOA keine überein-

419 Zum Begriff der Softwarekomponente vgl. Hansen/Neumann (2005), S. 225.

$420 \mathrm{Zu}$ den verschiedenen Kohäsionsarten vgl. auch Müller (2007a), S. 94ff.

$421 \mathrm{Vgl}$. Heutschi/Legner/Österle (2006), S. 367.

422 Allgemein wird unter dem Begriff der Kopplung das Ausmaß verstanden, mit dem eine Komponente mit einer anderen interagiert. Vgl. Hansen/Neumann (2005), S. 225; Tilkov/Starke (2007), S. 18. Der allgemeine Charakter dieser Definition impliziert, dass die Kopplungseigenschaft nicht nur im Zusammenhang mit Services, sondern auch in Verbindung mit Datenstrukturen, Funktionen sowie Klassen diskutiert wird.

423 Vgl. Wilms (2007), S. 566f.

424 Vgl. Herden et al. (2006), S. 52; Erl (2005), S. 291 und 297. 
stimmende Meinung. Während z. B. für MAHLBERG, NEWCOMER/LOMOW sowie SCHELP/STUTZ das Designprinzip der losen Kopplung eine herausragende Bedeutung für die Gestaltung von Services einnimmt,425 weisen HEUTSCHI/ LEGNER/ÖSTERLE diesem Prinzip die Bedeutung einer anzustrebenden, aber keinesfalls notwendigen Eigenschaft von SOA zu. ${ }^{426}$ Einen Schritt weiter gehen in diesem Zusammenhang TILKOV/STARKE, deren Auffassung nach eine lose Kopplung mit Blick auf die Schwierigkeit, eine asynchrone Kommunikation bzw. Interaktion zu implementieren, nicht immer wünschenswert sei.427 Nach OESTEREICH steht lose Kopplung im Kontext einer SOA sogar für einen überbewerteten und oft missverstandenen Mechanismus. ${ }^{428}$

\subsubsection{Prinzip der „Verwendung technischer und offener Industriestan- dards“}

Die Einhaltung der bereits thematisierten Gestaltungsprinzipien der Serviceorientierung hängt maßgeblich mit der Frage zusammen, inwieweit diese technisch umgesetzt werden können. Mit Blick auf diese Anforderung schreibt ein weiteres Gestaltungsprinzip vor, dass für die Entwicklung und die Nutzung von Services technische und offene Industriestandards einzusetzen sind, die eine hersteller- bzw. produktübergreifende Unterstützung erfahren.

Einem allgemeinen Verständnis folgend, definieren Standards einheitliche Eigenschaften oder Regeln für Objekte, mit denen sich eine Vergleichbarkeit oder Kompatibilität herstellen lässt. ${ }^{429}$ Aus dem Blickwinkel der IS betrachtet, werden Standards mit dem Ziel eingesetzt, Kosten für den Informationsaustausch zu reduzieren, die infolge der Nutzung heterogener Rechnernetze, Betriebssysteme, Benutzungsoberflächen und Anwendungssysteme entstehen. 430

Das Prinzip der technischen Standardisierung richtet sich auf die Bereitstellung einer geeigneten technischen Infrastruktur, mit deren Hilfe die im Rahmen einer SOA zu nutzenden Dienste entwickelt, veröffentlicht und aufgerufen werden können. ${ }^{431}$ Eine wichtige Aufgabe einer derartigen Infrastruktur liegt darin, die plattformspezifischen internen Datenformate und Kommunikationsprotokolle in gemeinsame bzw. standardisierte Formate und Protokolle zu transferie-

Vgl. Mahlberg (2007), S. 184; Newcomer/Lomow (2005), S. 75; Schelp/Stutz (2007), S. 67.

Vgl. Heutschi/Legner/Österle (2006), S. 368.

Vgl. Tilkov/Starke (2007), S. 18.

428 Vgl. Oesterreich (2007), S. 643. In seinem Beitrag liefert der Autor eine Gegenüberstellung der Anforderungen und Nachteile einer losen sowie festen Kopplung im Rahmen einer SOA. Vgl. Oesterreich (2007), S. 644.

Vgl. Buxmann (2001), S. 544ff.

430 Vgl. Buxmann (2001), S. 544.

431 Vgl. Heutschi/Legner/Österle (2006), S. 366. 
ren. ${ }^{432}$ Darüber hinaus sorgen technische Standards dafür, dass die Dienste über eine einheitliche Schnittstellenbeschreibung verfügen. Neben der Betonung technischer Standards wird im Zusammenhang mit der softwaretechnischen Umsetzung einer SOA insbesondere der Einsatz von offenen Standards propagiert, die von entsprechenden Non-Profit-Organisationen vorgeschlagen sowie weiterentwickelt werden. Offene Standards zeichnen sich dadurch aus, dass sie nicht der Marktmacht einer bestimmten Organisation unterliegen, i. d. R. entgeltlos oder eventuell über eine nominelle Gebühr genutzt werden können und je nach Anwendungsbereich beliebig erweiterbar sind. ${ }^{433}$

Zusammenfassend ist zu konstatieren, dass die in den vorangegangenen Ausführungen präsentierten Designprinzipien der Serviceorientierung keinesfalls unabhängig voneinander zu betrachten sind. Vielmehr bedingt die Existenz eines Prinzips das Vorhandensein eines weiteren. ${ }^{434}$ Exemplarisch ist der $\mathrm{Zu}$ sammenhang zwischen dem Prinzip der Serviceabstraktion und dem Prinzip der losen Kopplung zu nennen. ${ }^{435}$ Hierbei sind die Existenz einer Servicespezifikation sowie die damit zusammenhängende Erfüllung des Prinzips der Serviceabstraktion Voraussetzung dafür, dass sich eine lose gekoppelte Kommunikation zwischen den Diensten realisieren lässt. ${ }^{436}$

\subsubsection{Rollenkonzept einer SOA}

In den bisherigen Ausführungen blieb unberücksichtigt, auf welche Weise sich die Dienste in einer SOA nutzen lassen und welche Akteure dabei beteiligt sind. Damit Services in einer SOA angeboten, gefunden und schließlich genutzt werden können, ist das Zusammenspiel diverser Rollen von Bedeutung. ${ }^{437}$ Die im Rahmen einer SOA anfallenden Rollen und die zwischen diesen existierenden Kommunikationsbeziehungen lassen sich anhand des Rollenkonzepts einer SOA verdeutlichen, das in Abbildung 17 veranschaulicht ist. ${ }^{438}$

432 Vgl. Heutschi/Legner/Österle (2006), S. 366.

433 Charakterstisch für einen offenen Standard ist darüber hinaus, dass dieser das Ergebnis eines Abstimmungsprozesses darstellt und vor seiner Nutzung beliebig vervielfältigt werden kann. Zu den Eigenschaften offener Standards vgl. Heutschi/Legner/Österle (2006), S. 367.

434 Eine Übersicht zu den vielfältigen Beziehungsstrukturen der für ihn relevanten Prinzipien einer SOA liefert Erl (2005), S. 312ff.

$435 \mathrm{Vgl}$. hierzu die Abschnitte 3.3.2.1 und 3.3.2.2.

436 Vgl. hierzu auch Newcomer/Lomow (2005), S. 75.

437 Vgl. Melzer et al. (2007), S. 7.

438 Zum Zusammenspiel von Dienstanbieter, Dienstnutzer und Dienstverzeichnis vgl. Specht/Weisbecker/Spath (2006), S. 12. 


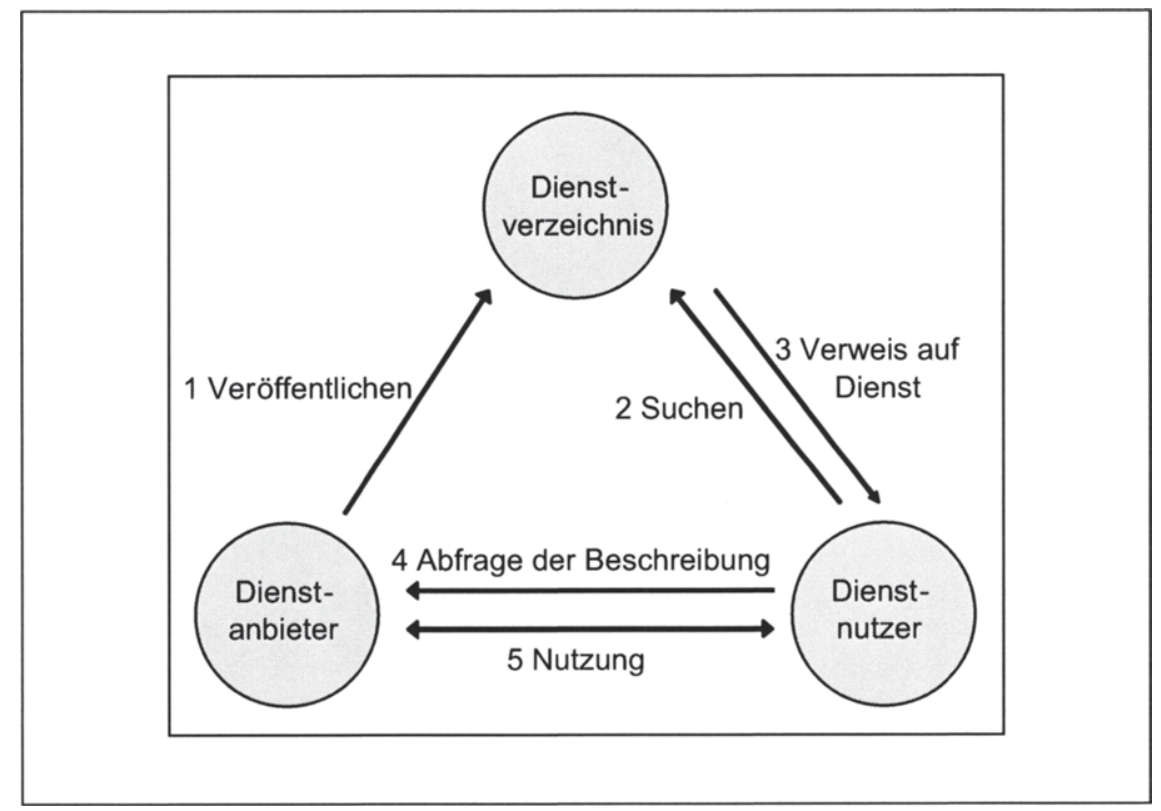

Abbildung 17: Rollenkonzept einer SOA 439

Wie aus dieser Abbildung hervorgeht, ist die Existenz eines Serviceanbieters von Bedeutung, der einem Servicenehmer einen Dienst oder mehrere Dienste zur Nutzung zur Verfügung stellt. Alternativ wird der Serviceanbieter auch als Service Provider bezeichnet. Die Aufgabe des Serviceanbieters liegt darin, eine geeignete Plattform zur Verfügung zu stellen, die einen Zugriff auf die von ihm veröffentlichten Dienste ermöglicht. Dabei ist es nicht erforderlich, dass der Service Provider die von ihm angebotenen Services selbst entwickelt. Alternativ kann ein Service Provider Dienste anbieten, die von einem dritten Akteur entwickelt wurden. 440

Für den Servicenehmer, der den bereitgestellten Dienst nachfragt, haben sich auch die Termini Servicenutzer, Servicekonsument und Service Requestor durchgesetzt. Der Service Requestor lässt sich mit dem Client in einer Client-

439 In Anlehnung an Melzer et al. (2007), S. 12.

440 In den Aufgabenbereich des Serviceanbieters fallen darüber hinaus die Aufrechterhaltung des Betriebs der Plattform und damit auch die Verfügbarkeit des Dienstes. Darüber hinaus ist vom Serviceanbieter Sorge zu tragen, dass die Sicherheit der Plattform gewährleistet ist. Dazu gehören Aufgaben zur Einrichtung der Authentifizierung und Authentisierung der Service. 
Server-Architektur vergleichen, der im Rahmen einer kooperativen Aufgabenabwicklung Funktionalitäten nutzt, die von einem Server erbracht werden. ${ }^{441}$

Damit sich die angebotenen Dienste von dem Servicenehmer auffinden lassen, wird mit dem Serviceverzeichnis, das auch als Service Broker oder Service Registry bezeichnet wird, eine weitere Rolle dazwischengeschaltet, die sowohl der Registrierung als auch dem Auffinden der Services dient. Um ein schnelles Auffinden der Dienste zu ermöglichen, ist es erforderlich, die Services in entsprechende Kategorien einzuordnen. In diesem Zusammenhang ist es möglich, dass eine Unternehmung, die sich für eine SOA entscheidet, mehrere Verzeichnisse betreiben kann. In einer derartigen Konstellation ließe sich ein Verzeichnis ausschließlich für die unternehmungsinterne Nutzung von Services einsetzen, während ein zweites Verzeichnis für die Registrierung und für das Auffinden von Diensten verwendet wird, die von Nutzern aufgerufen werden, die sich außerhalb der SOA-betreibenden Unternehmung befinden.

Für den Aufruf eines Services ist eine Reihe von Aktionen zu durchlaufen. 442 Damit ein Dienst von den potenziellen Nutzern gefunden werden kann, ist es im ersten Schritt notwendig, den Dienst in einem Serviceverzeichnis zu registrieren. ${ }^{443}$ Für diesen Zweck ist vom Anbieter des Services eine Servicespezifikation zu erstellen, die im nächsten Schritt im Verzeichnis veröffentlicht wird (1). Möchte ein potenzieller Dienstnutzer einen Service in Anspruch nehmen, sucht er anhand der in dem Verzeichnis existierenden Kriterien nach einem geeigneten Dienst (2). Erscheint ein Service als geeignet, erhält der potenzielle Servicenutzer die Adresse bzw. den Verweis auf die Servicespezifikation des von ihm nachgefragten Dienstes (3). ${ }^{444}$ Für diesen Zweck ist es wichtig, dass Standards eingehalten werden, die es dem Servicekonsumenten ermöglichen, auf die Schnittstellenbeschreibung des zu nutzenden Dienstes zuzugreifen (4). ${ }^{445}$ Unter Nutzung der Servicespezifikation lässt sich im letzten Schritt der betreffende Dienst aufrufen (5).

\subsection{Web Services zur Implementierung Serviceorientierter Anwendungen}

Neben SOA stellen auch die Web Services seit einigen Jahren einen viel beachteten Begriff in der Informatikliteratur dar. ${ }^{446}$ Unter den verschiedenen Möglichkeiten zur Implementierung Serviceorientierter Anwendungen, d. h. von Anwendungen, die auf einer SOA basieren, wird insbesondere der Einsatz von

441 Zum Client-Server-Konzept vgl. Fink/Schneidereit/Voß (2005), S. 43f.

442 Vgl. Melzer et al. (2007), S. 16.

443 Dafür ist es erforderlich, dass dieser Dienst bereits erstellt worden ist.

444 Vgl. Hammerschall (2005), S. 118.

445 Vgl. Hammerschall (2005), S. 117.

$446 \mathrm{Vgl}$. Eymann/Winter (2008), S. 70. 
Web Services als zukunftsweisend eingeschätzt. 447 Zunächst wird in Abschnitt 3.4.1 ein grundlegendes Verständnis zur Technologie der Web Services präsentiert. ${ }^{448} \mathrm{Um}$ Web Services im Rahmen einer SOA zu nutzen, sind verschiedene Standards einzusetzen. Abschnitt 3.4.2 widmet sich den Bausteinen der Web Service-Technologie.

\subsubsection{Grundverständnis zum Begriff des Web Services}

Bisher ist es den Autoren der zahlreichen Publikationen nicht gelungen, die verschiedenen Vorschläge zur Definition von Web Services zu einem einheitlichen Begriffsverständnis zu vereinen. 449 Mit Blick auf seinen integrierten und umfassenden Charakter zur Begriffsbestimmung eines Web Dienstes orientiert sich die vorliegende Arbeit an dem Definitionsvorschlag des Arbeitskreises ENTWICKLUNG WEB-SERVICEBASIERTER ANWENDUNGEN DER GESELLSCHAFT FÜR INFORMATIK (GI). ${ }^{450}$ Dem Arbeitskreis zufolge handelt es sich bei Web Services um ,selbst-beschreibende, gekapselte Softwarekomponenten, die eine Schnittstelle anbieten, über die ihre Funktionen entfernt aufgerufen und die lose durch den Austausch von Nachrichten miteinander gekoppelt werden können. Zur Er-

447 Vgl. hierzu Hofmann (2003), S. 27 und 28; Richter/Haller/Schrey (2005), S. 414. MELZER ET AL. bemerken in diesem Zusammenhang, dass die Entwicklung von Web Services maßgeblich das Interesse an SOA begünstigt habe. Vgl. Melzer et al. (2007), S. 337. BEIMBORN/WEITZEL stufen Web Services sogar als eine technische Revolution ein. Vgl. Beimborn/Weitzel (2003), S. 1360. Die Erwartungshaltung an Web Services wird auch bei ALT/HEUTSCHI/ÖSTERLE deutlich, die bemerken, dass Web Services als neue Zauberformel für die Integration von IS nach dem Vorbild einer „Lego-Wirtschaft“ gelten. Vgl. Alt/Heutschi/Österle (2003), S. 63. Damit spielen die Autoren auf den Modulcharakter von Web Services an.

448 Mit Blick auf die bei GABRIEL/BEIER diskutierte Abgrenzung der Begriffe „Technik“ und „Technologie“ wird in dieser Arbeit die Auffassung vertreten, bei Web Services handele es sich um eine Technologie. GABRIEL/BEIER führen in diesem Zusammenhang aus, dass eine Technologie neben der Technik auch die Anwendungs- und Nutzungsprozesse in Bezug auf die Technik umfasst. Vgl. Gabriel/Beier (2003), S. 84. Da Kenntnisse zur Nutzung von Web Services bekannt sind und auch in dieser Arbeit thematisiert werden, wird im Folgenden von der Web Service-Technologie gesprochen. Damit folgt diese Arbeit der Einschätzung weiterer Autoren. $\mathrm{Zu}$ diesen gehören z. B. Frotscher (2007), S. 489; Rebstock/Lipp (2003), S. 294; Oehler (2006), S. 106.

449 Vgl. Kossmann/Leymann (2004), S. 119. Auf die mannigfaltigen Versuche, Web Services zu definieren, und die daraus resultierende Konfusion verweist KÜSTER, indem er schreibt: „Wie bei allen schillernden Schlagworten bedeutet Web-Service für jeden etwas anderes und meistens etwas von allem“. Küster (2003), S. 6.

450 Mit Blick auf die bisher erfolgte synonyme Verwendung der Begriffe „Services“ und „Dienste“ werden im weiteren Verlauf dieser Arbeit zur Vermeidung einer sprachlichen Monotonie die Termini „Web Dienste“ und „Web Services“ genutzt. 
reichung universeller Interoperabilität werden für die Kommunikation die herkömmlichen Kanäle des Internets verwendet". 451

Wie aus diesem Definitionsansatz deutlich wird, verfügt ein Web Service über all diejenigen Eigenschaften eines Dienstes, die bereits in Abschnitt 3.3.1 thematisiert wurden. Web Services bieten für ihren Aufruf sowohl eine Schnittstelle als auch die softwaretechnische Realisierung der von ihnen bereitgestellten Funktionalität. Weiterhin werden von einem Web Service die in Abschnitt 3.3.2 präsentierten Gestaltungsprinzipien unterstützt. ${ }^{452}$ Die Eigenschaft der Selbstbeschreibung deutet auf die Erfüllung des Prinzips der „Abstraktion von der Implementierung". Übertragen auf den Einsatz von Web Services besagt dieses Prinzip, dass es für den Aufruf eines Web Services ausreicht, nur die im Vorfeld angelegten Beschreibungsmerkmale eines Web Dienstes wie Name, Funktionsbeschreibung, Dienstgüte etc. auszuwerten. Darüber hinaus folgen Web Services dem Prinzip der losen Kopplung, indem sie sich aufrufen lassen, ohne dass ihre Nutzer über Kenntnisse über die technische Realisierung des Dienstes verfügen müssen. In diesem Zusammenhang verweist die Eigenschaft der Kapselung darauf, dass ein Web Service eine unabhängige und in sich abgeschlossene Programmeinheit darstellt, die eine klar umrissene Aufgabe erfüllt.

\subsubsection{Bausteine einer Web Serviceorientierten Anwendung}

Zur Implementierung und Nutzung von Web Services konkurrieren derzeit eine Reihe unterschiedlicher Konzepte, Protokolle und Modelle, die infolge der Standardisierungsbestrebungen des WORLD WIDE WEB CONSORTIUM (W3C)453 SOwie der ORGANIZATION FOR THE ADVANCEMENT OF STRUCTURED INFORMATION STANDARDS (OASIS) ${ }^{454}$ entstanden sind sowie weiterentwickelt werden. ${ }^{455}$ Trotz der Heterogenität der zum Teil miteinander konkurrierenden Web Service-Standards ist zu konstatieren, dass die im Rahmen des Einsatzes von Web Services angestrebte Kommunikation zwischen den im Internet verteilten An-

451 Vgl. Herden et al. (2006), S. 69.

452 Siehe hierzu die Prinzipien bzw. Konzepte zur Gestaltung von Diensten in Abschnitt 3.3.2.

453 Zur Entstehung sowie zum Aufgabenbereich des W3C vgl. W3C (2009); Klettke/Meyer (2003), S. 20.

454 Zum Aufgabengebiet des Konsortiums vgl. OASIS (2008).

455 Vgl. Herden et al. (2006), S. 69. Während sich die Forschungsaktivitäten des W3CKonsortiums auf die Weiterentwicklung horizontaler bzw. industrieunabhängiger Standards richten, fokussiert OASIS die Entwicklung sowie Verbesserung vertikaler Standards. Vgl. Bettag (2001), S. 303. KüSTER bemängelt in diesem Zusammenhang eine „kaum mehr überschaubare Anzahl von Web-Service-Standards mit unterschiedlichen Zielsetzungen“. Küster (2003), S. 6. 
wendungen bzw. Objekten auf wenigen Grundlagenstandards basiert.456 Die Vorstellung dieser Standards ist Gegenstand der folgenden Ausführungen. Hierzu werden die Web Service-Standards SOAP (Abschnitt 3.4.2.2), WSDL (Abschnitt 3.4.2.2) und UDDI (Abschnitt 3.4.2.3) als wichtigste Bausteine einer Web Serviceorientierten-Anwendung thematisiert.

\subsubsection{Nachrichtenaustausch mit SOAP}

Eine wichtige Voraussetzung für den erfolgreichen Aufbau einer SOA ist die Integration von Services. ${ }^{457}$ Damit sich Services aufrufen lassen, muss ein geeignetes Protokoll zur Kommunikation mit den Diensten zur Verfügung stehen. Bei SOAP458 handelt es sich um ein Standardformat für die Übertragung von Nachrichten. ${ }^{459}$ Ein SOAP-Dokument gibt die Struktur der Nachrichten vor, in der der Aufruf an einen Web Service mit den benötigten Parametern spezifiziert ist. Handelt es sich um eine Antwortnachricht, enthält ein SOAP-Dokument die Ergebnisdaten. ${ }^{460}$ Zur Repräsentation der Daten kommt XML zum Einsatz, wobei zur Definition der Datentypen das standardisierte XML-Schema des W3C verwendet wird. 461

Damit der Zugriff auf einen Web Service sowie ein Nachrichtenaustausch zwischen zwei Web Services auf Basis von XML und über das Internet erfolgen kann, müssen geeignete Träger- bzw. Verpackungsprotokolle für den Nachrichtenaustausch zur Verfügung gestellt werden. Zur Übertragung der Daten werden folglich gängige Internetprotokolle wie beispielsweise Hypertext Transfer Protocol (HTTP), File Transfer Protocol (FTP), Simple Mail Transfer Protocol (SMTP) und Transmission Control Protocol (TCP) verwendet. 462 Aufgrund sei-

456 Vgl. hierzu Küster (2003), S. 6f.; Leyking/Ziemann/Loos (2006), S. 1037.

457 Vgl. Siebenhaar et al. (2008), S. 327.

458 Nachdem zu Beginn der Web Service-Technologie SOAP als Akronym für die Bezeichnung Simple Object Access Protocol diente, wird der Terminus SOAP heute nur noch als Eigenname verwendet. Vgl. Küster (2003), S. 7.

459 Vgl. Mintert (2005), S. 64; Leser/Naumann (2007), S. 405. Als Nachfolger des Middleware-Protokolls XML-RPC wurde SOAP im Jahr 2000 von MiCROSOFT entwickelt. Vgl. Hammerschall (2005), S. 112. Daraufhin erfolgte eine Weiterentwicklung von namhaften Firmen wie beispielsweise MicrosofT, HP, SAP und IBM.

460 Vgl. Hammerschall (2005), S. 118.

461 Daneben sind auch die Erstellung und der Einsatz eigener Datentypen möglich.

462 Vgl. Rebstock/Lipp (2003), S. 295. Der Einsatz etablierter Protokolle wie HTTP erleichtert die Übertragung von Nachrichten über Firewalls hinweg. Unter den Transportprotokollen wird dabei HTTP favorisiert. Vgl. Hammerschall (2005), S. 118. Bei der Datenübertragung über SOAP lassen sich mit dem Remote Procedure Call (RPC) und Document zwei Arten unterscheiden. Vgl. Küster (2003), S. 7. Während Document die Übertragung einer einzelnen Nachricht ermöglicht, handelt es sich bei RPC um einen entfernten Funktionsaufruf. 
nes XML-Bezugs ist SOAP ebenfalls unabhängig von der Plattform und der verwendeten Programmiersprache. Durch SOAP können nicht nur einzelne Parameter übergeben werden, sondern auch beliebige XML-Dokumente ausgetauscht werden. 463

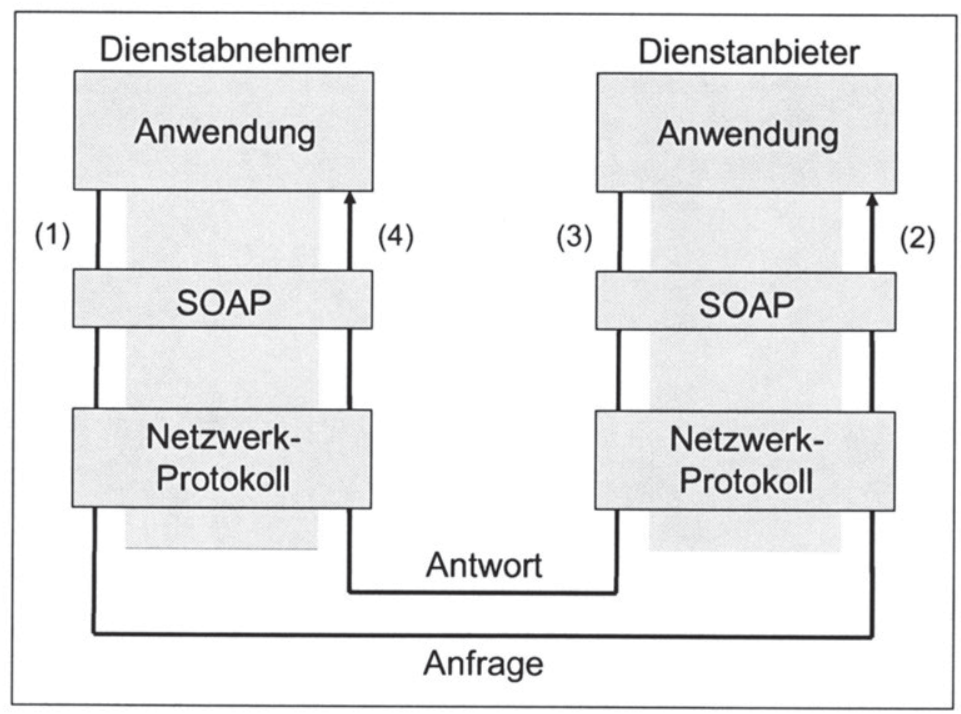

Abbildung 18: Aufruf eines Web Services

Zur Durchführung der Kommunikation kommt beispielsweise das RequestResponse-Konzept zum Einsatz. Wie in Abbildung 18 verdeutlicht wird, sendet 
der Dienstabnehmer unter Verwendung von HTTP einen Aufruf (Request), der auch als SOAP-Message bezeichnet wird, an eine Zielanwendung. ${ }^{464}$

Das SOAP-Dokument enthält eine Beschreibung, welche Funktionen auf der Zielanwendung vorliegen müssen. Das SOAP-Dokument kommt zum Einsatz, um alle relevanten Daten, die zur Anwendung der Funktion benötigt werden, an die Zielapplikation zu senden (1). Die im Rahmen der SOAP-Nachricht gekapselten Eingabeparameter werden von dem Web Service entpackt und an die jeweilige Anwendung weitergegeben (2). Nachdem die Eingabeparameter verarbeitet sind, wird das Ergebnis in Form einer SOAP-Antwort an den Sender zurückgeschickt (3). Dieser Teil der Kommunikation bildet den Response-Teil des Web Services. Im Anschluss wird das Ergebnis beim Dienstnehmer wieder entpackt (4).

Da es sich bei SOAP um ein XML-Dokument handelt, können die Bestandteile einer SOAP-Nachricht als Elemente bezeichnet und in Form einer geschachtelten Struktur angeordnet werden. Eine SOAP-Nachricht kann aus dem Envelope-, dem Header-, dem Body- sowie noch weiteren selbstdefinierten Elementen bestehen. Wie aus der Abbildung 19 hervorgeht, bildet das SOAPEnvelope-Element das übergeordnete Element bzw. das Wurzelelement, innerhalb dessen die eigentliche SOAP-Nachricht eingebettet ist. Das EnvelopeElement definiert den XML-Namensraum des Dokuments und beinhaltet neben dem Body- und optionalen Header-Element noch weitere benutzerdefinierte Elemente.

Das SOAP-Header-Element als untergeordnetes Element beschreibt die Metadaten einer Nachricht. Die darin enthaltenen Angaben informieren über das Routing, das verwendete Verschlüsselungsverfahren oder über die Herkunft der Nachricht. ${ }^{465}$ Ebenso lassen sich im Header-Element Meldungen einbetten, die im Fall einer fehlerhaften oder unvollständigen Datenübergabe aufgerufen werden. Im SOAP-Body werden alle Informationen untergebracht, die ein Web Service zur Aufgabenerfüllung benötigt. Folglich enthält der SOAP-Body beispielsweise den Namen der aufrufenden Funktion sowie die zur Aufgabenerfüllung notwendigen Daten. Handelt es sich um einen Methodenaufruf, so enthält der Body einer SOAP-Nachricht die erforderlichen Eingabeparameter. Bei einer Antwort sind im Body-Element die Rückgabedaten aufgeführt.

464 Das im Folgenden beschriebene Verfahren bietet nicht nur das Potenzial, eine rechnergestützte Kommunikation zwischen zwei Applikationen herzustellen, sondern eröffnet auch die Möglichkeit, mehrere Applikationen eines Zielsystems über einen Request anzusprechen. $\mathrm{Zu}$ diesem Zweck werden die Daten eines SOAP-Dokuments je nach Inhalt in entsprechende Methodenaufrufe transformiert. Die Verarbeitungsergebnisse der aufgerufenen Methoden lassen sich beispielsweise in einem Servlet bündeln und mit dem in diesem Abschnitt beschriebenen Übertragungsverfahren an die Quellanwendung übermitteln. 


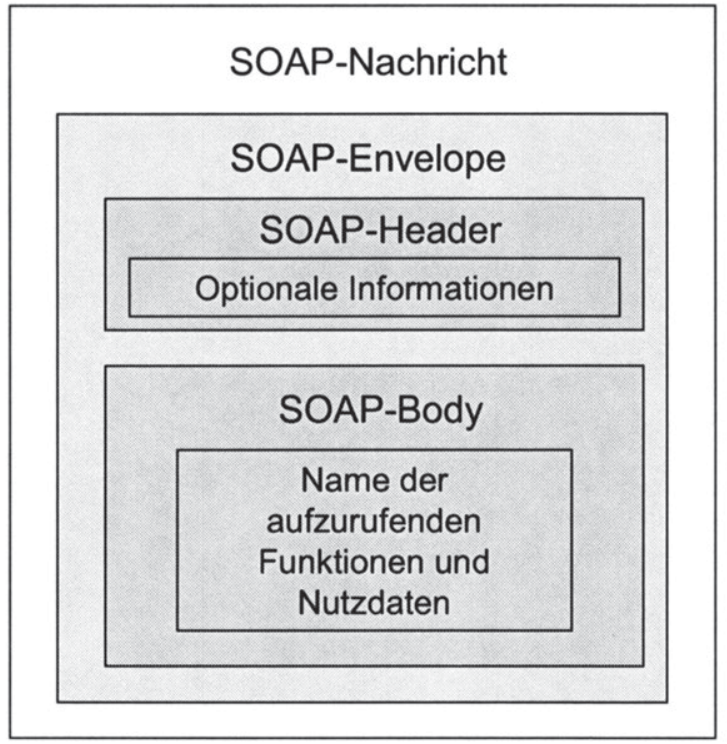

Abbildung 19: Struktur einer SOAP-Nachricht

\subsubsection{Schnittstellendefinition mit WSDL}

Wie beim Prinzip der „Abstraktion von der Implementierung“ deutlich wurde, ist die Beschreibung einer standardisierten, maschinenverständlichen Schnittstelle eine zentrale Voraussetzung für den Informationsaustausch zwischen einem Service-Nachfrager und einem -Anbieter. Um Web Services nutzen zu können, muss daher gewährleistet sein, dass die Dienste in einer angemessenen Weise spezifiziert und ihre Schnittstellen nach außen hin offen gelegt sind. 466 Im Rahmen der Spezifikation von Web Services ist beispielsweise festzulegen, über welche Methoden bzw. Funktionen ein Web Service verfügt, so dass der Aufruf einer anfragenden Instanz verarbeitet und in Form eines Ergebnisdokuments zurückgegeben werden kann.

Diese Aufgabe wird von einer Beschreibungs- bzw. Definitionskomponente übernommen, die den zweiten Baustein einer Web Serviceorientierten Anwen- 
dung repräsentiert. Der für diesen Zweck vom W3C entwickelte XML-Standard trägt die Bezeichnung Web Services Description Language (WSDL) ${ }^{467}$ Seit ihrem Erscheinen im Jahr 2000 hat sich WSDL heute als De-facto-Standard bei der Nutzung von Web Services etabliert. ${ }^{468}$ Mit Hilfe dieses Standards lässt sich eine Schnittstellenbeschreibung erstellen, in der alle für einen Serviceaufruf notwendigen technischen Informationen abgelegt werden, die von einem Client für einen Aufruf des Dienstes benötigt werden. ${ }^{469}$

$$
\begin{aligned}
& <\text { definitions }> \\
& \begin{array}{l}
<\text { types }>\ldots \text { <types }> \\
<\text { message name }=\text { "Message } 1 ">\ldots<\text { /message }> \\
<\text { portType }>\ldots</ \text { portType }> \\
\hline<\text { binding }>\ldots</ \text { binding }> \\
<\text { service }>\ldots</ \text { service }>
\end{array} \\
& <\text { definitions }>
\end{aligned}
$$

\section{Abbildung 20: Aufbau eines WSDL-Dokuments}

Anhand der Abbildung 20 lassen sich die wichtigsten Konzepte sowie die Struktur einer WSDL-Schnittstellenbeschreibung veranschaulichen. Wie in der Abbildung zu erkennen ist, besteht ein WSDL-Dokument sowohl aus einem abstrakten als auch einem konkreten Teil. Während das types-Element, die message-Elemente sowie das portType-Element für den abstrakten Teil eines WSDL-Dokuments stehen, handelt es sich bei dem binding-Element und dem service-Element um Vertreter des konkreten Teils einer WSDL-Datei. 470

Das definitions-Element ist das Wurzelelement eines WSDL-Dokuments. Innerhalb des definitions-Elements werden der Name und der Namensraum des Web Services sowie der Namensraum der verwendeten Standards als Attribut-

468 Dass WSDL die am weitesten verbreitete Definitionssprache im Web Service-Umfeld ist, wird deutlich bei Stiemerling (2002), S. 439. Der Ursprung von WSDL datiert auf das Jahr 2000, als im September dieses Jahres WSDL von den Unternehmungen ARIBA, IBM und Microsoft präsentiert wurde. Vgl. Küster (2003), S. 9. Während WSDL als Candidate Recommendation bereits in der Version 2.0 existiert, erfolgt eine produktive Verwendung heute auf Basis der Version 1.1.

469 Vgl. Leser/Naumann (2007), S. 405. Wie in der Literatur üblich, werden im Folgenden die Begriffe WSDL-Datei und WSDL-Dokument synonym verwendet.

470 Vgl. Liebhart (2007), S. 16. 
werte festgelegt. Die Aufgabe des types-Elements liegt darin, alle Datentypdefinitionen festzulegen, die zum Austausch der Nachrichten bzw. der messages erforderlich und nicht im W3C-Standard zum XML-Schema enthalten sind. ${ }^{471}$ Innerhalb der message-Elemente werden die Nachrichten festgelegt, die bei einem SOAP-Aufruf übertragen werden. Hierbei ist für jede Anfrage sowie Antwort eine separate Nachricht zu definieren. 472

Mit Hilfe des portType-Elements lassen sich alle Operationen beschreiben, die der Web Service anbietet. ${ }^{473}$ Die Methoden werden in einem gesonderten Element mit der Bezeichnung operations definiert. Dabei unterstützt WSDL vier Nachrichtentypen. ${ }^{474}$ Als Alternativen stehen die Nachrichtentypen OneWay, Request-Response, Solicit-Response und Notification zur Verfügung. Der One-Way-Nachrichtentyp ist dadurch gekennzeichnet, dass ein Web Service nach dem Eintreffen einer Nachricht des Client nicht antwortet, während beim Request-Response dies der Fall ist. Im Rahmen des Solicit-Response sendet der Server eine Nachricht an den Client, worauf von diesem eine Antwort folgt. Beim Notification-Nachrichtentyp wird hingegen eine Antwort des Client nicht erwartet.

Das binding-Element als Konzept des konkreten Teils einer WSDLSchnittstellenbeschreibung wird genutzt, um das Nachrichtenformat und das Transportprotokoll vorzugeben, das für die Übertragung der Aufrufe zum Einsatz kommt. $475 \mathrm{Im}$ Rahmen des service-Elements werden Informationen aufgeführt, die für den Zugriff auf den Web Service notwendig sind. Die Informationen betreffen z. B. Angaben zur verwendeten Netzwerkadresse sowie Portnummer.

\subsubsection{Veröffentlichung mit UDDI}

Damit ein Web Service von möglichst vielen Servicenehmern genutzt werden kann, ist eine geeignete Plattform bereitzustellen, mittels dieser sich Web Services in einem Netzwerk bekannt machen und auffinden lassen. ${ }^{476}$ Diese Aufgaben werden von einem Verzeichnisdienst mit der Bezeichnung Universal Desc-

471 Dies hat zur Folge, dass sich für den jeweiligen Web Service zugeschnittene Datentypen nutzen lassen, die mit Hilfe der Datentypen des XML-Schemas erstellt werden.

472 Erfordert der Web Service einen Eingabeparameter, der nach seiner Verarbeitung zu einem Rückgabewert führt, sind folglich zwei Nachrichten zu definieren.

473 Vgl. Reinefeld/Schintke (2004), S. 131.

474 Vgl. hierzu Hammerschall (2005), S. 116; Liebhart (2007), S. 17. Welcher Nachrichtentyp zum Einsatz kommt, hängt von der Reihenfolge der messages ab. Folgt einem inputElement ein output-Element, deutet dies auf einen Request-Response-Nachrichtentyp. Liegt nur ein input-Element vor, handelt es sich um einen One-Way-Nachrichtentyp. Als Bindungen kommen neben SOAP auch HTTP GET sowie HTTP POST in Frage.

476 Vgl. Brehm/Haak/Gómez (2008), S. 493. 
ription, Discovery and Integration (UDDI) erfüllt, der den dritten Baustein einer Web Serviceorientierten Anwendung darstellt.477 Von einem UDDIVerzeichnisdienst werden zwei Funktionen bereitgestellt. ${ }^{478}$ Zum einen lassen sich mit Hilfe eines UDDI-Verzeichnisdienstes Web Services registrieren. 479 Zum anderen stellt ein Verzeichnisdienst einen Zugriffsmechanismus zur Verfügung, der es ermöglicht, einen Dienst für eine potenzielle Nutzung aufzufinden. 480 In diesem Zusammenhang werden einerseits direkte Suchanfragen unterstützt, die durch die Eingabe eines oder mehrerer Schlagworte ausgelöst werden können. Andererseits können Web Services anhand von Klassifikationskategorien aufgefunden werden, indem der Nutzer eines UDDI in einer vom Betreiber des Verzeichnisdienstes vorab definierten Aufteilung bzw. Systematisierung der angebotenen Dienste nach dem Vorbild eines Drill-Down navigieren kann. ${ }^{481}$

Die Katalogisierung der in einem UDDI registrierten Web Services erfolgt nach dem Vorbild eines Branchenbuchs. ${ }^{482}$ Die Verzeichnisse sind dabei in drei Bereiche aufgeteilt, die als White Pages, Yellow Pages und Green Pages bezeichnet werden. ${ }^{483}$ In Anlehnung an das Branchenverzeichnis GELBE SEITEN beschreiben die Yellow Pages eine Gruppe von Services und klassifizieren diese anhand von Industriezweigen. Yellow Pages erlauben die Suche über Schlagwörter oder durch einen Zugriff auf die Klassifikationskategorien. ${ }^{484}$ Green Pages informieren über die technische Ausgestaltung eines angebotenen Web Services. I. d. R. wird auf die dazugehörende WSDL-Datei verwiesen. Ferner sind Informationen zum Geschäftsmodell einer Unternehmung diesem Informationsbereich zugeordnet. In den White Pages sind alle Angaben enthalten, um mit einem Dienstanbieter interagieren zu können. Neben einem Namensregister führen White Pages Kontaktinformationen der Service Provider

477 Vgl. Beimborn/Weitzel (2003), S. 1362f. Die Bezeichnung UDDI geht auf den Namen eines Projekts zurück, das von den Softwarefirmen MICROSOFT und IBM initiiert wurde. Als Ziel dieses Projekts wurde die Implementierung eines globalen, standardisierten Verzeichnisses zum Auffinden von Web Services definiert.

478 Vgl. hierzu Hammerschall (2005), S. 117; Brehm/Haak/Gómez (2008), S. 493.

479 Der UDDI-Standard sieht in diesem Zusammenhang eine entsprechende Application Programming Interface (API) vor.

480 In Anlehnung an die API zum Auffinden von Web Services definiert der UDDIStandard ebenfalls eine Inquiry-API. Als alternativer Standard für das Auffinden von Web Services lässt sich die Web Service Inspection Language (WS-Inspection) nutzen. Eine Vorstellung dieses Standards liefern Ballinger et al. (2001) und Brittenham (2002).

481 Vgl. Küster (2003), S. 10.

$482 \mathrm{Vgl.} \mathrm{Kossmann/Leymann} \mathrm{(2004),} \mathrm{S.} 122$.

483 Vgl. Bettag (2001), S. 304.

484 Als gebräuchlichste Klassifikationsschemata können entweder das North American Industry Classification System (NAICS) oder die Universal Standard Products and Services Codes (UNSPSC) zur Anwendung kommen. Vgl. Küster (2003), S. 10. 
auf und ähneln damit einem Telefonbuch. ${ }^{485}$ Darüber hinaus liefern die White Pages weitere Identifikationsmerkmale wie die Data Universal Numbering System-Nummer (DUNS), die zur eindeutigen Identifizierung von Unternehmungen genutzt wird.

Die Struktur und die Funktionalität eines Verzeichnisdienstes sind in einem UDDI-Schema spezifiziert. Das Schema definiert das Modell aller Entitäten, die zur Beschreibung der in einem UDDI-Verzeichnisdienst angebotenen Web Services verwendet werden können. Von Bedeutung sind dabei folgende Entitäten. ${ }^{486}$ Mit Hilfe der businessEntity wird die Unternehmung oder die Geschäftseinheit beschrieben, die den Web Service anbietet. Darüber hinaus wird diese Entität verwendet, um einen Web Service im Verzeichnisdienst anzumelden. Dies erfolgt, indem eine neue businessEntity erstellt wird, der im zweiten Schritt die WSDL-Schnittstellenbeschreibung des jeweiligen Services zugeordnet wird. Die businessService-Entität dient zur Beschreibung einer Menge zusammengehörender Web Services, die von einer businessEntity angeboten werden. Die Entität bindingTemplate enthält die technischen Informationen, die für den Zugriff auf einen Web Service notwendig sind. Unter Einsatz von tModel (taxonomy model) werden die businessEntities und businessServices kategorisiert. Für diesen Zweck definieren tModels eine Taxonomie im Verzeichnisdienst, in der businessEntities und businessServices abgelegt und gefunden werden können.

Der UDDI-Verzeichnisdienst lässt sich einsetzen, um private Verzeichnisse zu implementieren, die beispielsweise auf eine unternehmungsinterne Anwendung der angebotenen Dienste ausgelegt sind. Gleichzeitig haben Web ServiceAnbieter auch die Möglichkeit, ihre Dienste in ein öffentliches Verzeichnis einzutragen, damit diese von einem möglichst großen Anwenderkreis genutzt werden können. ${ }^{487}$ Als letzte Variante sind Zwischenformen denkbar, bei denen Web Services sich in branchenspezifischen, semi-öffentlichen Verzeichnissen registrieren lassen. 488

\subsection{Zusammenfassung und Ableitung eines integrierten SOA- Verständnisses}

Die Ausführungen des vorliegenden Kapitels hatten zum Ziel, grundlegende konzeptionelle und technische Facetten zum derzeit intensiv diskutierten Ansatz einer SOA darzustellen. Ausgehend vom Begriff der integrierten Informations-

Vgl. Küster (2003), S. 10.

486 Vgl. Hammerschall (2005), S. 118.

487 Eine Übersicht $\mathrm{zu}$ einigen ausgewählten öffentlichen Verzeichnissen liefert z. B. KÜSTER. Vgl. Küster (2003), S. 11. 
verarbeitung ließen sich in Abschnitt 3.1 zwei zentrale Problemfelder identifizieren, die eine integrierte Informationsverarbeitung gefährden. Während ein Problemfeld die hohen Kosten der Systementwicklung und Systemintegration thematisierte, hat ebenso das Problem einer mangelnden IT-Unterstützung der Geschäftsprozesse einer Unternehmung das Interesse am Konzept einer SOA in den vergangenen Jahren gefördert.

Aufbauend auf den Ausführungen ausgewählter Literaturbeiträge, die in Abschnitt 3.2 in drei Auslegungsgruppen einer SOA unterteilt und diskutiert wurden, fokussierte Abschnitt 3.3 die Softwareservices als konzeptionelles und technisches Fundament einer SOA. Dabei wurde in diesem Kapitel sowohl auf die Darstellung der Bestandteile eines Services als auch auf die Prinzipien Wert gelegt, die bei der Gestaltung von Services zu berücksichtigen sind. Die Frage nach einer geeigneten softwaretechnischen Implementierung einer SOA wurde in Abschnitt 3.4 erörtert, indem mit den Web Services eine von der SOALiteratur favorisierte Technologie zur Realisierung einer SOA thematisiert wurde. Zum Abschluss des vorliegenden Kapitels erfolgt in diesem Abschnitt die Präsentation eines integrierten Begriffsverständnisses einer SOA, das auf den Inhalten der Abschnitte 3.1 bis 3.4 basiert.

In Abschnitt 3.2 wurde herausgestellt, dass sich SOA vor dem Hintergrund aktueller Literatur aus drei Blickwinkeln betrachten lässt. Hierbei stehen sich die Autoren, die den konzeptionellen Ansatz einer SOA favorisieren, denjenigen gegenüber, die die konkrete technische Implementierung einer SOA betonen. 489 Der vorliegende Abschnitt verfolgt das Ziel, die verschiedenen Sichtweisen zu einem integrierten SOA-Begriffsverständnis zusammenzuführen. Dabei nehmen die folgenden Ausführungen zu der Frage Stellung, ob es sich bei SOA tatsächlich um ein neues Paradigma bzw. um einen Paradigmenwechsel in der Informationsverarbeitung handelt. 490 Der Terminus des Paradigmenwechsels folgt dabei der Begriffsauslegung von KUHN, der einen Paradigmenwechsel in allgemeiner Weise mit der Veränderung von Überzeugungen, Werten und Verfahrensweisen, die von den Mitgliedern einer bestimmten Gemeinschaft vertreten werden, gleichsetzt. 491

Mit Blick auf die in Abschnitt 3.3 präsentierten Auslegungen ist die Gemeinsamkeit zu konstatieren, dass die Serviceorientierung einen fundamentalen Pfeiler des SOA-Ansatzes darstellt. An dieser Stelle ist darauf hinzuweisen, dass die Idee, ein IS in Module bzw. Softwarebausteine zu zerlegen und diese in Form gekapselter Funktionen einer möglichst großen Nutzerzahl zur Verfügung zu

489 Zur technisch-geprägten Auslegung einer SOA vgl. Abschnitt 3.2.2. Wie in Abschnitt 3.2 deutlich wurde, vertreten die Repräsentanten der konzeptorientierten Auslegung einer SOA die Auffassung, bei SOA handele es sich um ein Architekturkonzept (Abschnitt 3.2.1) und/oder um ein Rollenkonzept (Abschnitt 3.3.3). 
stellen, kein Novum in der Informatik darstellt. Eine identische Zielsetzung wurde bereits in der Diskussion um die Entwicklung und den Einsatz komponentenorientierter IS geäußert. 492 Im Gegensatz zu einem monolithischen System wird die Funktionalität in einem komponentenorientierten System von verschiedenen klar abgegrenzten Modulen bzw. Bausteinen erbracht, die eine Teilfunktionalität abbilden und zur Nutzung anbieten. ${ }^{493}$ Damit stellt der Einsatz einer SOA im Vergleich zur Nutzung grob strukturierter Softwarepakete sowie monolithischer Applikationen die Möglichkeit in Aussicht, voneinander abgegrenzte Aufgaben in Form von Services anzubieten, die sich je nach Einsatzzweck zu einem erweiterten Gesamtsystem frei kombinieren lassen. ${ }^{494}$

Vor dem Hintergrund der in Abschnitt 3.1.1 vorgestellten Integrationstypen der Geräte-, Prozess-, Funktions-, und Datenintegration ist festzustellen, dass die Verwendung von Services gleichbedeutend mit der Existenz einer weiteren Integrationsschicht ist, die für eine integrierte Informationsverarbeitung zum Einsatz kommen kann. Mit Blick auf das Servicemodell in Abschnitt 3.3.1 lässt sich feststellen, dass eine oder mehrere Funktionen Bestandteil eines Services sein können. Services sollen wiederum als Aktivitäten zu einer Prozesskette zusammengesetzt und - so der Anspruch einer SOA - in verschiedenen Prozessen ausgeführt sowie von verschiedenen Servicenachfragern genutzt werden. Dies hat zur Folge, dass zwischen den Ebenen der Funktions- und Prozessintegration eine weitere eingefügt werden kann, die für die Integration von Softwareservices steht. Eine vollständige Übersicht über alle Integrationsschichten einer SOA präsentiert Abbildung 21.

Neben dem Servicebegriff liegt auch im Architekturterminus ein zentrales Charakteristikum einer SOA. Angelehnt an den Architekturbegriff nach FOEGEN/BATTENFELD, ${ }^{495}$ steht SOA damit für die Regeln sowie Richtlinien, die bei der Entwicklung einer Serviceorientierten Anwendung zu berücksichtigen sind. ${ }^{496}$ Mit Blick auf das Architekturverständnis, das HERDEN ET AL. favorisieren, lässt sich SOA in gleicher Weise als ein Konzept auffassen, das zur Gestaltung, Wartung, Dokumentation und Nutzung eines dienstorientierten IS angewendet werden kann. ${ }^{497}$ Dies hat zur Konsequenz, dass sowohl statische als auch dynamische Aspekte bei der Entwicklung und beim Betrieb einer SOA zu

492 Diese Einschätzung teilen auch HÖß ET AL., indem sie ausführen, dass sich die Grundideen einer SOA in der komponentenbasierten Softwareentwicklung wieder finden lassen. Vgl. Höß et al. (2007), S. 40. Vgl. Hansen/Neumann (2005), S. 226.

$494 \mathrm{Vgl}$. Hansen/Neumann (2005), S. 532. Dieser Aspekt wird von LIEBHART plakativ als „Dienste statt Applikationen“ bezeichnet. Vgl. Liebhart (2007), S. 9.

496 Diese wurden als Designprinzipien in Abschnitt 3.3.2 thematisiert.

497 Vgl. Herden et al. (2006), S. 1. 
berücksichtigen sind. ${ }^{498}$ Mit Blick auf die statischen Eigenschaften einer SOA ist zu bemerken, dass einerseits die Designprinzipien die zentralen Richtlinien einer SOA darstellen. Die dynamische Perspektive zeichnet sich hingegen durch die Kommunikationsbeziehungen in einer SOA aus.

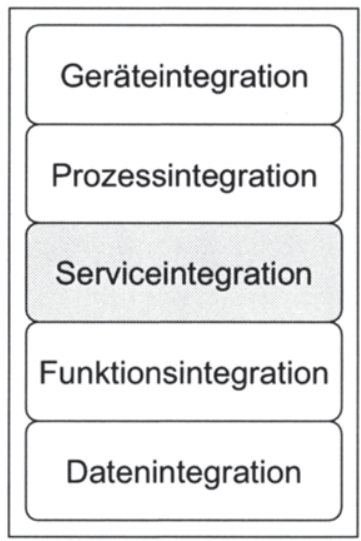

Abbildung 21: Erweiterung der Integrationstypen um die Ebene der Serviceintegration

Wird Kommunikation als der Austausch von Nachrichten zwischen einem Sender und einem Empfänger verstanden, ${ }^{499}$ liegt das Fundament für den Informationsaustausch innerhalb einer SOA in der Existenz eines Serviceanbieters, eines Servicenachfragers sowie eines Serviceverzeichnisses. ${ }^{500}$ Damit ein Nachrichtenaustausch zwischen den Servicenachfragern und -anbietern zustande kommt, ist die Voraussetzung zu erfüllen, dass ein Servicevertrag zur Verfügung gestellt wird, der in Form von Metadaten alle wesentlichen Informationen zum Aufruf eines Services enthält.

Der Einsatz einer SOA bietet im Vergleich zu alternativen Konzepten punktuelle Erweiterungen, die eine Auseinandersetzung mit diesem Ansatz rechtfertigen. Diese Feststellung wird auch von TILKOV/STARKE gestützt, deren Auffassung zufolge die wichtigste Innovation einer SOA im Vergleich zu bereits bewährten Ideen, wie z. B. der Verwendung präziser Schnittstellen zur losen Kopplung von Softwaremodulen sowie der Einsatz von XML, in dem Einbezug

498 Siehe hierzu die Ausführungen zum Architekturbegriff in Abschnitt 3.2.1.

499 Vgl. Gabriel/Beier (2003), S. 36.

500 HERDEN ET AL. sprechen in diesem Zusammenhang von dem Grundmodell der SOA. Vgl. Herden et al. (2006), S. 6. 
von Metadaten liegt. Diese ermöglichen neben der Beschreibung der funktionalen Anforderungen insbesondere die präzise Definition nichtfunktionaler Eigenschaften eines Services und deren Auswertung zur Laufzeit. ${ }^{501}$ Hierin liegt ein wichtiges Differenzierungsmerkmal einer SOA im Vergleich zu alternativen Ansätzen, die sich mit der Gestaltung integrierter IS auseinandersetzen.

Darüber hinaus zeichnet sich das SOA-Konzept durch eine stärkere Berücksichtigung des Internets aus, das die Rolle einer Kommunikationsplattform einnimmt, innerhalb dieser verteilte Dienste in Anspruch genommen werden können, die sich beispielsweise in Form von Web Services realisieren lassen. ${ }^{502}$ Der Einsatz eines Netzwerks als Medium für den Aufruf der Web Services ermöglicht es, die Nutzung nicht nur auf diejenigen Dienste zu beschränken, die von einer Unternehmung implementiert und freigegeben wurden. Unternehmungen können ihre Services in gleichem Maße unternehmungsexternen Nutzern zur Verfügung stellen, wie sie die von unternehmungsexternen Anbietern bereitgestellten Dienste nutzen können. 503

Durch den Einsatz standardisierter und offener Protokolle, wie es im Zusammenhang mit dem Prinzip der „Verwendung technischer und offener Standards“ gefordert wird, ${ }^{504}$ soll die Möglichkeit eröffnet werden, eine Interaktion zwischen den heterogenen Anwendungssystemen einer Unternehmung herzustellen, die auf verschiedenen Programmiersprachen, Infrastrukturen oder Plattformen basieren.505 Gerade der Einsatz der Web Service-Technologie, die sich des XML-Standards bedient, ist eine wichtige Neuerung im Vergleich zu alternativen Integrationsansätzen. ${ }^{506}$ Dabei stellt die Nutzung der Web ServiceStandards wie SOAP, WSDL und UDDI die Möglichkeit in Aussicht, nicht nur Dienste zu beschreiben, sondern auch ihre Auffindbarkeit und Komposition innerhalb einer SOA zu unterstützen.507 Wie bei der Vorstellung der Bausteine einer Web Serviceorientierten Anwendung deutlich wurde, fällt zur Implementierung und Nutzung derartiger Services neben XML den Netzwerkprotokollen in ihrer Funktion als Transportprotokolle eine wichtige Bedeutung zu.

Wie die Einschätzungen einiger Autoren belegen, wird die Implementierung und Nutzung einer SOA mit dem Einsatz von Web Services gleichgesetzt. ${ }^{508}$ An dieser Stelle ist darauf hinzuweisen, dass es sich hierbei ausschließlich um eine Empfehlung handeln darf. Keineswegs setzt der Betrieb einer SOA den Einsatz

Vgl. Tilkov/Starke (2007), S. 36.

Vgl. Beimborn/Weitzel (2003), S. 1360.

Vgl. Hansen/Neumann (2005), S. 532.

Vgl. Abschnitt 3.3.2.3.

Vgl. Rieks (2006), S. 7. Siehe hierzu auch das Beispiel bei Küster (2003), S. 6.

STREIBICH spricht in diesem Zusammenhang sogar von einer entscheidenden Neuerung.

Vgl. Streibich (2008), S. 73.

$507 \mathrm{Vgl}$. Eymann/Winter (2008), S. 70.

508 Vgl. hierzu Abschnitt 3.2.2. 
von Web Services voraus. ${ }^{509}$ Auch wenn die Implementierung beim Software Engineering eine wichtige Phase darstellt, greift die Definition, die ausschließlich die technischen Aspekte bzw. den Einsatz einer bestimmten Technologie zur softwaretechnischen Umsetzung einer SOA fokussiert, zu kurz. ${ }^{510}$ Damit entfällt die Möglichkeit, eine SOA als bestimmte Technologie oder einen Technologiestandard aufzufassen. 511

Mit Blick auf die fachlichen Anwendungsmöglichkeiten einer SOA ist festzuhalten, dass im Vergleich zu alternativen Integrationsansätzen der Fokus bei SOA auf die Geschäftsprozesse einer Unternehmung gelegt wird. ${ }^{512} \mathrm{Da}$ die reine IT-Sicht nicht im Vordergrund steht, lässt sich folglich eine integrierte Perspektive sowohl auf die Geschäftsprozesse als auch auf die Anwendungssysteme einer Unternehmung einnehmen. ${ }^{513}$ In diesem Zusammenhang verspricht der Einsatz einer SOA, die Geschäftsprozesse, die sowohl innerbetrieblich als auch unternehmungsübergreifend ausgeführt werden, durch den Einsatz geeigneter Services zu flexibilisieren und/oder zu automatisieren. Vor diesem Hintergrund bietet SOA das Potenzial, nicht nur technische sondern auch organisatorische Grenzen zu beseitigen. ${ }^{514}$ Die Ausrichtung auf die Geschäftsprozesse einer Unternehmung, die sich mit Hilfe zusammengefügter Services automatisieren und/oder flexibilisieren lassen sollen, macht gleichzeitig deutlich, dass eine SOA immer eine unternehmungsspezifische Ausprägung aufweist. In Analogie zu alternativen Konzepten in der Wirtschaftsinformatik wie z. B. zum DWHKonzept, 515 das zur Gestaltung unternehmungsindividueller analyseorientierter IS genutzt wird, lässt sich eine SOA ebenfalls nicht als fertige Lösung von einem Softwareanbieter erwerben und in einer Unternehmung einsetzen. 516

509 Alternativ lassen sich zur Implementierung einer SOA z. B. die Technologien Common Object Request Broker Architecture (CORBA), Distributed Component Object Model (DCOM) oder das Java-Pendant Remote Method Invocation (RMI) nutzen. Gemein haben diese Ansätze, dass sie einen Zugriff auf entfernte Objekte ermöglichen. Die Grundlagen zu diesen Technologien thematisiert z. B. HAMMERSCHALL. Vgl. Hammerschall (2005), S. 89ff., 127ff. und 190f. Zu den Einschränkungen dieser Lösungsvarianten vgl. Rieks (2006), S. 6. Dass sich eine SOA auf Basis von CORBA implementieren lässt, verdeutlicht das SOA-Projekt der Unternehmung CREDIT SUISSE. Vgl. Klostermeier (2008), S. 2.

510 Die Auffassung, dass es sich bei einer SOA um ein technologieneutrales Konzept handelt, wird auch von Höß ET AL. geteilt. Vgl. Höß et al. (2007), S. 40.

511 Vgl. hierzu auch Holtschke/Heier/Hummel (2009), S. 120; Thomas/Leyking/Dreifus (2007), S. 44; Reinheimer et al. (2007), S. 8.

512 Vgl. Bauler et al. (2007), S. 57.

513 Vgl. Tilkov/Starke (2007), S. 36.

514 Vgl. Bauler et al. (2007), S. 57.

515 Zum DWH-Konzept vgl. Gabriel/Röhrs (2003), S. 340ff.; Gluchowski/Gabriel/Dittmar (2007), S. $117 \mathrm{ff}$.

516 Vgl. Streibich (2008), S. 73. 
Da SOA als Ausprägung eines komponentenorientierten IS betrachtet werden kann und die Grundidee derartige Systeme keine Neuheit in der Informatik darstellt, ist im Ergebnis festzustellen, dass SOA, aus einer konzeptionellen Perspektive betrachtet, keinen revolutionären Ansatz darstellt.517 Stattdessen handelt es sich bei SOA um einen Ansatz, der die konsequente Fortführung etablierter Konzepte der Objekt- und Komponentenorientierung verfolgt, 518 um eine integrierte Informationsverarbeitung $\mathrm{zu}$ ermöglichen. $\mathrm{Zu}$ diesen gehören beispielsweise die Prinzipien der Abstraktion, Modularisierung, Standardisierung und Verteilung. ${ }^{519}$ Mit Blick auf die von KUHN formulierten Merkmale eines Paradigmenwechsels ist die Ansicht, bei SOA handele es sich um ein neues $\mathrm{Pa}$ radigma in der Informatik, daher abzulehnen.

Stattdessen wird in dieser Arbeit SOA als abstraktes Architekturkonzept aufgefasst, bei dem wiederverwendbare und voneinander unabhängige Softwarekomponenten, die bestimmten Designprinzipien unterliegen und eine gezielte Funktion erfüllen, zur Unterstützung einer integrierten Informationsverarbeitung zum Einsatz kommen. Auf fachlicher Seite richtet sich der Betrieb einer SOA auf das Ziel, innerbetriebliche und unternehmungsübergreifende $\mathrm{Ge}$ schäftsprozesse durch den Einsatz zusammengefügter Services zu automatisieren und/oder zu flexibilisieren und auf diese Weise eine integrierte Informationsverarbeitung zu ermöglichen. Zur Implementierung der Services lassen sich diverse Technologien einsetzen, wobei insbesondere der Einsatz der Web Service-Technologie propagiert wird. ${ }^{520}$

517 Vgl. Laartz (2008), S. 72.

518 Vgl. Beverungen/Knackstedt/Müller (2008), S. 221.

519 Diese Einschätzung wird auch plakativ in der Aussage von TERZIDIS/SURE/BrELAGE deutlich. Die Autoren bemerken: ,SOA is nearly as old as computing itself from a technical perspective“. Vgl. Terzidis/Sure/Brelage (2008), S. 76.

520 Eine alternative, pragmatische Synthese ausgewählter Definitionsvorschläge zu SOA präsentieren Tilkov/Starke (2007), S. 17. 
Alexander Pastwa - 978-3-631-75488-7

Downloaded from PubFactory at 01/11/2019 04:20:29AM

via free access 


\section{Architekturmodell für Serviceorientierte Berichtsprozesse}

In Kapitel 2 wurde herausgearbeitet, dass ein prozessorientiertes Berichtswesen auf eine integrierte Informationsverarbeitung angewiesen ist. Hierzu wurde in Kapitel 3 mit SOA ein aktuell diskutiertes Architekturkonzept vorgestellt, das sich losgelöst von einem bestimmten Anwendungsbereich insbesondere zur Flexibilisierung und/oder Automatisierung von Geschäftsprozessen und damit zur Gestaltung einer effizienten und effektiven Informationsverarbeitung einsetzen lässt. Der in dieser Arbeit betrachtete Anwendungsbereich von SOA richtet sich auf die Informationsverarbeitung im betrieblichen Berichtswesen. Im Besonderen wird dabei der Frage nachgegangen, wie sich mit Hilfe dieses Konzepts Serviceorientierte Berichtsprozesse (SOBP) gestalten und eine unternehmungs- und/oder applikationsübergreifende Verarbeitung von Geschäftsund Finanzinformationen auf Basis zusammengefügter Dienste implementieren lassen. 521

In diesem Zusammenhang ist nach THOME festzuhalten, dass sich die betriebliche Informationsverarbeitung sowohl durch den systematischen Aufbau ihrer Komponenten als auch durch die Abfolge der Schritte auszeichnet, die zur Verarbeitung der Daten und Informationen durchgeführt werden müssen, um ihre semantisch korrekte Aufbereitung und Darstellung zu ermöglichen. ${ }^{522}$ Das dieser Feststellung zugrunde liegende Erfordernis, die betriebliche Informationsverarbeitung anhand einer statischen und einer dynamischen Perspektive zu betrachten, geht mit dem SOA-Verständnis der vorliegenden Arbeit einher. Vor dem Hintergrund der Tatsache, dass eine SOA die Zielstruktur einer ISArchitektur definiert und „Strukturen“ sowohl statische als auch dynamische Aspekte implizieren, widmet sich dieses Kapitel zunächst der statischen Perspektive einer SOA. Entsprechend werden die grundlegenden Strukturen der Systemkomponenten einer SOA erörtert.

Der Aufbau des vorliegenden Kapitels gestaltet sich wie folgt. In Abschnitt 4.1 wird der Begriff „Serviceorientierter Berichtsprozess“ (SOBP) eingeführt. Um einen SOBP auf Basis einer SOA zu gestalten, ist es erforderlich, ein Architekturmodell aufzustellen, das der Entwicklung und Realisierung einer derartigen Anwendung zugrunde liegt. Ein geeignetes Architekturmodell zur Gestaltung von SOBP zu entwerfen, ist von großer Bedeutung, da ein SOBP einen für diese Arbeit zentralen Prozess darstellt, der sich auf Basis einer SOA automatisieren und/oder flexibilisieren lässt. Vor diesem Hintergrund sind Überlegungen zum architektonischen Aufbau einer SOA grundsätzlicher Art und unabhängig

Im Folgenden wird die Abkürzung „SOBP“ für Einzahl und Mehrzahl verwendet. 
von der $\mathrm{zu}$ unterstützenden prozessorientierten Anwendung. In Abschnitt 4.2 wird hierzu das SOA-Ebenenmodell entwickelt, das sich zur Gestaltung und Ausführung Serviceorientierter Prozesse heranziehen lässt.

Die Ausführungen des Abschnitts 4.3 widmen sich der Frage, welche Erweiterungen am bestehenden SOA-Architekturmodell vorzunehmen sind, um die Informationsverarbeitungsprozesse im betrieblichen Berichtswesen durch den Einsatz einer SOBP zu verbessern. Als Erweiterung des Architekturmodells wird mit der Extensible Business Reporting Language (XBRL) ein Standard vorgestellt, der das Potenzial zur Gewährleistung der semantisch korrekten Verarbeitung von Geschäfts- und Finanzinformationen in einer SOA mit sich bringt.

Das Kapitel schließt in Abschnitt 4.4 mit einer zusammenfassenden Würdigung des um XBRL erweiterten SOA-Architekturmodells.

\subsection{Begriff eines Serviceorientierten Berichtsprozesses}

Untersucht man vorhandene Publikationen zum Reporting und zu SOA, so ist festzustellen, dass eine einschlägige Definition des Begriffs „Serviceorientierter Berichtsprozess" (SOBP) bislang fehlt. Vor diesem Hintergrund hat der vorliegende Abschnitt das Ziel, eine solche zu entwickeln. Das Verständnis für den Terminus eines SOBP basiert dabei auf den in den Abschnitten 2.2.2 und 3.3 erarbeiteten Grundlagen der Berichtsprozesse und Softwaredienste.

Unter einem SOBP wird in dieser Arbeit ein Berichtsprozess verstanden, bei dem die Aktivitäten und Teilprozesse der Kernprozesse Informationsbeschaffung und Berichtsproduktion, -verteilung sowie -aufnahme mit Hilfe von Services implementiert werden. Derartige Services werden im Folgenden als Berichtsservices bzw. Berichtsdienste bezeichnet. Berichtsservices haben die Verarbeitung der im Rahmen eines Berichtsprozesses anfallenden Geschäfts- und Finanzinformationen zum Gegenstand. Sie sind nicht auf einen bestimmten Funktionsumfang beschränkt. Dies bedeutet, dass ein Berichtsservice sowohl lesende als auch schreibende Operationen durchführen kann, die bei einem oder mehreren Berichtsprozessen zum Einsatz kommen, wobei ein Berichtsservice im Hinblick auf seine Nutzungshäufigkeit flexibel ist. D. h., ein bestimmter Berichtsservice lässt sich sowohl bei regelmäßig ausgeführten Berichtsprozessen als auch bei unregelmäßig stattfindenden Prozessen verwenden.

$\mathrm{Da}$ es sich bei einem Berichtsservice um eine Ausprägung eines Services handelt, verfügen Berichtsdienste über alle in Abschnitt 3.3.1 präsentierten Merkmale sowie Bestandteile eines Services. Darüber hinaus folgen sie den in Abschnitt 3.3.2 thematisierten Prinzipien der Serviceorientierung. Die Einhaltung der Prinzipien „lose Kopplung“, „Verwendung technischer und offener Standards“ und „Abstraktion von der Implementierungslogik“ hat zur Folge, dass sich die Berichtsservices als modulare Softwarekomponenten zu einem Be- 
richtsprozess kombinieren und durch die Bereiststellung einer geeigneten ITInfrastruktur ausführen lassen. Dabei setzen sie auf den bestehenden Daten der zum Einsatz kommenden Berichtssysteme und Datenformate auf. ${ }^{523}$ Die Designprinzipien „lose Kopplung“ und „Abstraktion von der Implementierungslogik" stellen in diesem Zusammenhang in Aussicht, dass sich Berichtsservices in einem oder in mehreren Kernprozessen des Berichtswesens einbinden lassen.

Durch die Komposition von Berichtsservices wird zum einen die Verarbeitung von berichteten Informationen entlang einer Berichtskette flexibilisiert und/oder automatisiert, zum anderen bietet der Einsatz von Berichtsservices, die auf Basis offener und technischer Standards implementiert wurden, das Potenzial, Berichtsservices unternehmungsexterner Anbieter in einen bestehenden Berichtsprozess einzubinden. Als Beispiel für einen derartigen Service lässt sich ein Aktieninformationsdienst eines externen Anbieters anführen, der nach seinem Aufruf die Servicenutzer mit aktuellen Aktienkursen ausgewählter Unternehmungen versorgt. ${ }^{524}$ Durch die Integration unternehmungsexterner Berichtsservices lassen sich Geschäfts- und Finanzinformationen anwendungsund/oder unternehmungsübergreifend verarbeiten.

\subsection{SOA-Ebenenmodell als geeignetes Architekturkonzept für Serviceori- entierte Berichtsprozesse}

Damit sich Services in einer SOA aufrufen und zu einer dynamischen Prozesskette kombinieren lassen, muss eine entsprechende Architektur bereitgestellt werden, mit der das Rollenkonzept einer SOA umgesetzt wird.525 Während die in Abschnitt 3.3.3 präsentierten Rollen und ihre Beziehungen die konzeptionelle Grundlage einer SOA darstellen, wird im Folgenden das architektonische Fundament einer SOA erarbeitet.

Losgelöst von den verschiedenen kommerziellen sowie nicht kommerziellen Softwareprodukten, die aktuell zur Implementierung einer SOA angeboten werden, nehmen die Ausführungen dieses Abschnitts zu den Basisstrukturen einer SOA Stellung. ${ }^{526}$ In diesem Zusammenhang geht es um die Frage, welche zentralen Komponenten prägend für das Architekturverständnis einer SOA sind und

523 Dabei lassen sich durch Berichtsservices sowohl operative bzw. disaggregierte als auch aggregierte Daten verarbeiten.

Vgl. Bettag (2001), S. 302.

Vgl. hierzu auch Beimborn/Mintert/Weitzel (2002), S. 277.

526 Eine Zusammenstellung entsprechender nicht kommerzieller Produkte zur Implementierung einer SOA liefern Bauler et al. (2007), S. 58ff. 
auf welche Weise sich diese Komponenten zu einer SOA-Referenzarchitektur bzw. zu einem SOA-Architekturmodell zusammenfügen lassen. 527

Hinsichtlich der Zusammensetzung eines SOA-Architekturmodells zeichnen sich die in der Literatur präsentierten Vorschläge eher durch Gemeinsamkeiten als durch Unterschiede aus. Mehrheitlich stößt man dabei auf die Darstellung einer SOA-Referenzarchitektur als Ebenenmodell. Als Ergebnis einer Analyse ausgewählter Beiträge lässt sich ein Architekturmodell definieren, das aus vier Ebenen besteht. 528 Dieses Modell setzt sich aus der Ebene der Anwendungssysteme (Abschnitt 4.2.1), der Ebene der Services (Abschnitt 4.2.2), der Ebene der Prozesse (Abschnitt 4.2.3) und der Ebene der Frontendsysteme (Abschnitt 4.2.4) zusammen. In den folgenden Abschnitten werden die Eigenschaften und Aufgaben der vier, übereinander angeordneten Ebenen des SOA-Architekturmodells sowie ihre Komponenten und Beziehungen konkretisiert. Dabei werden nicht nur technische, sondern auch fachliche Gesichtspunkte beleuchtet. Um die Ausführungen durchgängig übersichtlich zu halten, erfolgt eine systematische Erweiterung des Architekturmodells von der Ebene der Anwendungssysteme bis hin zur Ebene der Frontendsysteme.

Die Ausprägung des Architekturmodells sowie die Bezeichnungen der einzelnen Komponenten sind dabei angelehnt an den Vorschlag von HEUTSCHI/ LEGNER/ÖSTERLE. ${ }^{529}$ Charakteristisch für das fokussierte Architekturmodell ist, dass es sich in eine anwendungsbezogene und eine anwendungsneutrale Sicht aufteilen lässt. Während bei der anwendungsbezogenen Sicht die Konzepte und Komponenten der jeweiligen Architekturebene eine auf den jeweiligen Einsatzzweck ausgerichtete Ausprägung aufweisen - beispielsweise zur Unterstützung ausgewählter Geschäftsprozesse - zeichnen sich die Bausteine der anwendungsneutralen Perspektive durch einen allgemeingültigen Charakter aus. Sie lassen sich daher losgelöst von einem spezifischen Einsatzzweck betrachten.

\subsubsection{Ebene der Anwendungssysteme}

Auf der Ebene der Anwendungssysteme befinden sich alle betrieblichen Applikationen, welche die fachlichen Funktionen ebenso wie die Datenbestände zur Verfügung stellen, die Gegenstand der Serviceimplementierung sein können. Als zu nutzende Anwendungssysteme kommen beispielsweise CustomerRelationship-Management-Systeme (CRM-Systeme) und Supply-Chain-

527 Die Begriffe Referenzarchitektur und Architekturmodell werden im Folgenden synonym verwendet.

528 Vgl. hierzu die Vorschläge eines Architekturmodells von Erl (2005), S. 282; Heutschi/ Legner/Österle (2006), S. 362ff.; Liebhart (2007), S. 26ff.; Newcomer/Lomow (2005), S. 59; Josuttis (2008), S. 135ff.

529 Vgl. Heutschi/Legner/Österle (2006), S. $362 \mathrm{ff}$. 
Management-Systeme (SCM-Systeme) in Frage. Bei der Gestaltung eines SOBP ist vor allem die Anbindung von Enterprise-Resource-Planning-Systemen (ERP-Systemen) sowie allen weiteren Vorsystemen einer Berichtsanwendung von Bedeutung. Sie halten die Geschäfts- und Finanzinformationen vor, die zur Erfüllung der Berichtszwecke notwendig sind. Darüber hinaus kommen auch die in dem DWH einer Berichtsanwendung enthaltenen konsolidierten Daten als Ergebnis einer multidimensionalen Aufbereitung der Daten aus den operativen Systemen - als Datenlieferanten in einer derartigen Serviceorientierten Anwendung in Betracht. ${ }^{530} \mathrm{Ziel}$ ist es hierbei, sowohl unternehmungsinterne als auch unternehmungsexterne Anwendungssysteme im Rahmen einer SOA anzubinden. ${ }^{531}$ Abbildung 22 verdeutlicht die Komponenten der Ebene der Anwendungssysteme innerhalb des SOA-Architekturmodells.

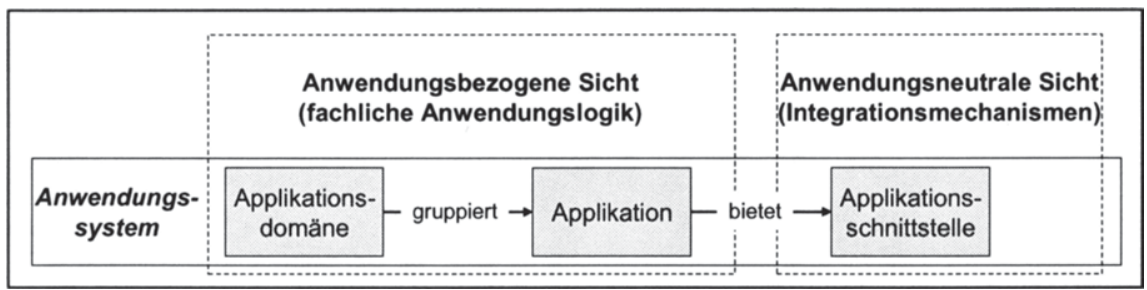

Abbildung 22: Ebene der Anwendungssysteme ${ }^{532}$

Wie in der Abbildung 22 zu erkennen ist, lassen sich auf der Ebene der Anwendungssysteme drei Architekturkomponenten unterscheiden. Im Mittelpunkt dieser Ebene stehen die Applikationen, die im Rahmen einer SOA weiter betrieben werden sollen. Damit Services die Daten der Applikationen verarbeiten können, ist es erforderlich, dass die Anwendungssysteme eine entsprechende Schnittstelle zur Verfügung stellen, die die Dienste nutzen können. Ferner lassen sich die einzelnen Anwendungssysteme zu Applikationsdomänen zusammenfassen. Ziel der Domänenbildung ist es, fachlich zusammengehörige Operationen bzw. Funktionen sowie Datenobjekte, die von den betriebenen IS zur Verfügung ge-

530 Auf die Bedeutung von DWH-Systemen als Datenlieferanten in einer SOA verweisen z. B. DitTMAR und KRUCZYNSKI. Vgl. Dittmar (2007), S. 135ff.; Kruczynski (2006), S. 25.

531 Vgl. Heutschi/Legner/Österle (2006), S. 363.

532 In Anlehnung an Heutschi/Legner/Österle (2006), S. 364. Im Vergleich zum Vorschlag von HEUTSCHI/LEGNER/ÖsTERLE wird die Komponente der Applikationsdomäne in dieser Arbeit als Bestandteil der Ebene der Anwendungssysteme betrachtet, da der Fokus dieser Komponente gerade darin liegt, die Funktionalitäten der betriebenen Anwendungssysteme nach fachlichen Gesichtspunkten zu Domänen zu strukturieren. 
stellt werden und sich für eine Serviceimplementierung eignen, redundanzfrei zu gruppieren. 533

Nach der Analyse der Schnittstellen sind in einem nächsten Schritt sämtliche Operationen bzw. Funktionen zu identifizieren, die von den betriebenen IS zur Verfügung gestellt werden.

Während in dem hier präsentierten Architekturmodell die Anwendungssysteme das Fundament einer SOA darstellen, schlägt LIEBHART vor, mit der Schicht der Virtualized Infrastructure eine weitere Ebene zu betrachten. ${ }^{534}$ Diese ist unterhalb der Ebene der Anwendungssysteme positioniert. Auf ihr sind alle technischen sowie infrastrukturellen Ressourcen zum Betrieb sämtlicher SOA-Komponenten (wie Prozessoren, Arbeitsspeicher, Netzwerkverbindungen, etc.) bereitzustellen und zu steuern. 535 Dieser Sichtweise zufolge repräsentiert die Ebene der Anwendungssysteme die Datensicht sowie die fachliche Ressourcensicht innerhalb des SOA-Architekturmodells, während die darunter liegende Schicht der Virtualized Infrastructure auf eine technische Ressourcensicht abzielt. Da die technischen Ressourcen einen integralen Bestandteil von Anwendungssystemen darstellen, wird die von LIEBHART vorgenommene Separierung in zwei Ebenen in der vorliegenden Arbeit nicht weiter verfolgt.

\subsubsection{Ebene der Services}

Oberhalb der Ebene der Anwendungssysteme befindet sich die Ebene der Services. Auf der Serviceebene werden alle Dienste betrachtet, die der SOAbetreibenden Unternehmung entweder infolge einer externen Bereitstellung oder durch Eigenentwicklung zu einem gegebenen Zeitpunkt zur Verfügung stehen. Als Ausgangspunkt dient das Servicemodell, das in Abschnitt 3.3.1 erörtert wurde. Damit sich die Funktionalitäten von Services voneinander abgegrenzen lassen, wird in Abschnitt 4.2.2.1 eine gebräuchliche Kategorisierung von Diensten vorgestellt. Die Servicearten werden daraufhin als logische Komponente in das Architekturmodell aufgenommen. Als weitere Komponente der Ebene der Services werden in Abschnitt 4.2.2.2 die Middlewaredienste thematisiert. Im Anschluss erfolgt in Abschnitt 4.2.2.3 die Einbindung der zuvor betrachteten Komponenten in die Ebene der Services.

533 Siehe hierzu die Ausführungen in Abschnitt 5.2.2.3.

534 Vgl. Liebhart (2007), S. 27.

535 Vgl. Liebhart (2007), S. 27. 


\subsubsection{Servicekategorien}

Eine Klassifizierung von Servicetypen lässt sich anhand der Kategorien Datenservices, Funktionsservices und Prozessservices durchführen. ${ }^{536}$ Datenservices haben die Aufgabe, die Daten der IS, auf die die Dienste zugreifen, zu verarbeiten. Im Fokus können sowohl Stammdaten als auch Prozessdaten stehen. ${ }^{537}$ Als alternative Bezeichnungen für Datenservices werden in der Literatur die Termini Bestandsservices, Basisservices oder datenzentrierte Services verwendet. 538 Ihr Leistungsumfang umfasst die so genannten CRUD-Operationen. ${ }^{539}$ Das Akronym CRUD steht dabei für die Operationen Create, Read, Update und Delete. ${ }^{540}$ Aus der Wortwahl ist ersichtlich, dass die Datenoperationen an den Befehlsumfang von Datenbanksprachen, wie z. B. der Structured Query Language (SQL), angelehnt sind. Von Bedeutung sind in diesem Zusammenhang die Sprachelemente bzw. Befehle der Data-Manipulation-Language (DML) von SQL. 541

536 Diese Klassifizierung verwenden auch Luhmann/Meister/Wulff (2007), S. 345. Neben den Daten-, Funktions- und Prozessservices werden so genannte Interaktionsservices als weitere Dienstkategorie genannt. Vgl. Lauer/Juwig (2007), S. 267. In dieser Arbeit wird die Auffassung vertreten, dass sich Interaktionsservices nicht als Servicekategorie verstehen lassen, da sie nicht dem Servicebegriff entsprechen, der einer SOA zugrunde liegt. Vgl. hierzu auch Hess/Humm/Voß (2006), S. 399. Interaktionsservices ermöglichen den verschiedenen Nutzern einer SOA einen Zugriff auf die Dienste. Die Anwender können in diesem Zusammenhang verschiedene Medien bzw. Kanäle in Anspruch nehmen, zu denen beispielsweise Intranet- sowie Internet-Portale gehören. Die Interaktionsservices sind daher gesondert von den vorherigen drei Servicekategorien zu betrachten, da ihre Services keine Schnittstellen im softwaretechnischen Sinne darstellen. Vielmehr stehen sie für die verschiedenen Benutzungsschnittstellen bzw. Zugriffskomponenten, die einem Anwender zur Inanspruchnahme eines Daten-, Funktions- und Prozessservice zur Verfügung stehen.

Vgl. Krafzig/Banke/Slama (2007), S. 88; Lauer/Juwig (2007), S. 266.

538 Da „Basisservices“ auch als übergeordneter Terminus für alle elementaren Operationen verwendet wird und damit sowohl die Daten- als auch die Funktionsservices einschließt, lassen sich die Datenservices auch als Basisservices i. e. S. verstehen. Vgl. Höß et al. (2007), S. 40. Um eine eindeutige begriffliche Zuordnung zu gewährleisten, werden in dieser Arbeit die Basisservices auch als Datenservices bezeichnet, von den Funktionsservices abgegrenzt und als eigene Servicekategorie betrachtet. Vgl. Hess/Humm/Voß (2006), S. 399.

540 Zu den CRUD-Operationen vgl. Keller (2007), S. 362.

541 Zur Einführung in die DML-Funktionen von SQL vgl. z. B. Gabriel/Röhrs (2003), S. 64ff.; Pernul/Unland (2003), S. 403ff. 
Mit Hilfe der Bestandsservices lassen sich neue Datensätze anlegen (Create) sowie bestehende Datensätze auslesen (Read). ${ }^{542}$ Darüber hinaus können bestehende Datensätze aktualisiert (Update) und gelöscht (Delete) werden. Festzuhalten ist, dass es sich bei diesen vier Operationen um elementare Operationen handelt. ${ }^{543}$ Sie dienen der Erzeugung von Datensichten sowie der Durchführung von Datenpflegeoperationen, wobei Konsistenzbedingungen einzuhalten bzw. zu überwachen sind. ${ }^{544}$ Darüber hinaus zeichnen sich Bestandsservices durch einen hohen Grad an Eigenständigkeit bzw. Unabhängigkeit aus. ${ }^{545}$ Beispiele für Bestandsservices sind die Buchung eines Umsatzbetrags oder die Historisierung von Daten.

Funktionsservices zielen darauf $a b$, die verschiedenen Geschäftsfunktionen einer Unternehmung softwaretechnisch abzubilden.546 In Anlehnung an KRAFZIG/BANKE/SLAMA lassen sich Funktionsservices auch als logikzentrierte Services bezeichnen. Derartige Service zeichnen sich durch die Kapselung von Verarbeitungsanweisungen aus, die sich sowohl für einfache und komplexe Berechnungen als auch für Geschäftsregeln einsetzen lassen. Funktionsservices können auf den Bestandsdiensten aufsetzen, indem sie diese zur Datenlieferung sowie - nach erfolgter Verarbeitung der Dateneingabe - zur Datenrückschreibung nutzen. ${ }^{547}$ Auch lassen sie sich als atomare Dienste aufrufen, die unabhängig von anderen Services verwendet werden und die erforderlichen lesenden und schreibenden Operationen selbständig ausführen. Ein Beispiel einer auf Basis eines Funktionsservices zu unterstützenden Geschäftsfunktion ist die Bonitätsprüfung eines Kunden oder die Erstellung von Abrechnungen. 548

Prozessservices, die auch als prozesszentrierte Services, Business-Services und als Geschäftslogik-Services bezeichnet werden, ${ }^{549}$ sind darauf ausgerichtet, die einzelnen Funktions- und Bestandsservices zu koordinieren und zu einem übergeordneten Service zusammenzufügen. ${ }^{550}$ Mit Hilfe von Prozessservices

542 Käme ein relationales Datenbanksystem zur Durchführung der CRUD-Operationen zum Einsatz, ist an dieser Stelle darauf hinzuweisen, dass die Create-Operation, d. h. das Anlegen von Datensätzen, dem „Insert Into“-Befehl aus dem DML-Befehlssatz von SQL entspricht und nicht mit dem „Create Table“-Befehl aus dem DDL-Befehlssatz von SQL verwechselt werden sollte. Die übrigen Operationen, $d$. h. die RUD-Operationen, lassen sich mit Hilfe des Select-, Update- und Delete-Befehls von SQL implementieren.

Vgl. Höß et al. (2007), S. 40.

544 Vgl. Hess/Humm/Voß (2006), S. 399.

545 Vgl. von Henning (2007), S. 351 und 353.

546 Vgl. Hess/Humm/Voß (2006), S. 399.

547 Vgl. Lauer/Juwig (2007), S. 266.

$548 \mathrm{Vgl}$. Hess/Humm/Voß (2006), S. 399.

549 Vgl. Höß et al. (2007), S. 40; Luhmann/Meister/Wulff (2007), S. 345.

550 Vgl. Lauer/Juwig (2007), S. 267; von Henning (2007), S. 351; Hess/Humm/Voß (2006), S. 399. 
lassen sich Geschäftsprozessmodule ausführen.551 Darüber hinaus können Prozessservices wiederum als Teilprozesse in einen übergeordneten Prozessservice eingebunden werden.

Im Vergleich zu den Basis- und den Funktionsservices zeichnen sich Prozessservices auf der einen Seite dadurch aus, dass sie statusbehaftet sind, da sie ihren Status für die sie aufrufende Instanz jeweils selbst kontrollieren und verwalten. ${ }^{552}$ Auf der anderen Seite weisen prozesszentrierte Services im Vergleich zu den Basis- und Funktionsservices einen stärkeren Prozessbezug bzw. eine höhere Prozessspezifität auf, da sie sich aus verschiedenen Daten- und Funktionsservices zusammensetzen, die zur Ausführung einer bestimmten Aktivität oder eines Teilprozesses verknüpft wurden.

\subsubsection{Middlewaredienste}

Innerhalb der Ebene der Services erfüllen die Middlewaredienste eine wichtige Funktion. Ihnen obliegt die Aufgabe der Daten-, Funktions- und Präsentationsintegration, womit Middlewaredienste als Integrationskomponente des Architekturmodells zu verstehen sind. Sie sind für die Verknüpfung der verschiedenen Dienste zuständig und ermöglichen die Kopplung zwischen den Diensten und den Anwendungen über die entsprechenden Schnittstellen. Der dritte Aufgabenbereich richtet sich auf die Verbindung der Services mit den zum Einsatz kommenden Frontendsystemen. ${ }^{553}$ Mit Blick auf die technische Ausprägung der Middlewaredienste kommt zur Erfüllung aller drei Aufgabenbereiche typischerweise ein Enterprise-Service-Bus (ESB) zum Einsatz.

Ein ESB enthält Instrumente zur sicheren und garantierten Meldungsübertragung, zum Logging und zur Autorisierung. Routingmechanismen sowie Management- und Überwachungstools werden ebenfalls von einem ESB bereitgestellt. 554 Ein ESB übernimmt die Details der Kommunikation, die erforderlich sind, damit Servicenehmer mit Serviceanbietern in Kontakt treten können. 555 Eine wichtige Funktion ist in diesem Zusammenhang die Überwindung heterogener Technologien, da i. d. R. ein Servicenutzer in einer anderen Programmiersprache implementiert ist als der Serviceprovider. Darüber hinaus sorgt ein ESB für die technische Konnektivität zwischen allen Komponenten einer SOA. Dies betrifft auch die notwendigen Netzwerk- und Protokolldetails. ${ }^{556}$

Vgl. Roth (2007), S. 99.

552 Vgl. Krafzig/Banke/Slama (2007), S. 96.

553 Vgl. Abschnitt 4.2.4.

554 Vgl. Liebhart (2007), S. 136; Tilkov/Starke (2007), S. 34.

555 Vgl. Tilkov/Starke (2007), S. 34; Buxmann/Hess/Widjaja (2007), S. 1320.

556 Vgl. Tilkov/Starke (2007), S. 34. 


\subsubsection{Beziehungen zwischen den Komponenten der Serviceebene}

Abbildung 23 verdeutlicht, wie sich das in Abschnitt 3.3.1 diskutierte Servicemodell, die Servicekategorien (Abschnitt 4.2.2.1) sowie die Middlewaredienste (Abschnitt 4.2.2.2) in die Ebene der Services einbinden lassen.

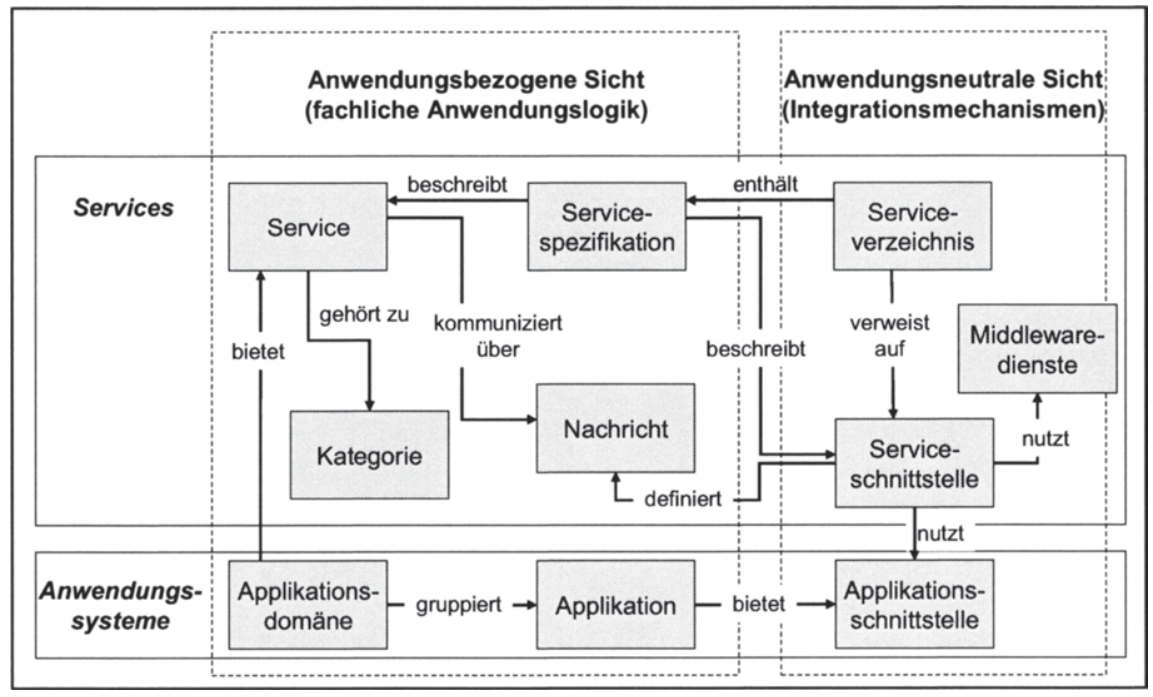

Abbildung 23: Erweiterung des SOA-Architekturmodells um die Ebene der Services

Zur Nutzung der Dienste ist innerhalb der Serviceebene eine Komponente einzubinden, die es ermöglicht, die aktuell zur Verfügung stehenden Dienste zu registrieren, zu verwalten und aufzufinden. Diese Funktionalität wird von einem Serviceverzeichnis übernommen. 557 Das Serviceverzeichnis enthält die Servicebeschreibungen, die zum Auffinden der Dienste zur Laufzeit herangezogen werden. 558 Darüber hinaus verweist das Serviceverzeichnis auf die Serviceschnittstellen. Wie in der Abbildung 23 dargestellt, setzen die Serviceschnittstellen auf Schnittstellen der Anwendungssysteme auf, um ihre spezifischen Funktionalitäten zu erbringen. Vor dem Hintergrund ihrer Bedeutung für die

$557 \mathrm{Zu}$ alternativen Bezeichnungen sowie zu den Aufgaben eines Serviceverzeichnisses innerhalb des SOA-Rollenkonzepts vgl. Abschnitt 3.3.3. CONRAD ET AL. empfehlen in diesem Zusammenhang die Service-Registry von dem Service-Repository, das zur Unterstützung des Entwicklungsprozesses zum Einsatz kommt, zu unterscheiden. Vgl. hierzu Conrad et al. (2006), S. 90.

Vgl. Conrad et al. (2006), S. 90. 
konzeptionelle Gestaltung Serviceorientierter Prozesse wird in Erweiterung des in Abschnitt 3.3.1 diskutierten Servicemodells die Servicekategorie als logische Komponente ins Architekturmodell aufgenommen. ${ }^{559}$ Die Verbindung zwischen den Komponenten „Applikationsdomäne“ und „Services“ weist ebenfalls auf eine logische Beziehung hin, die zur Aussage hat, dass sich aus den gruppierten Funktionen und Datenobjekten einer Applikationsdomäne Services identifizieren und kapseln lassen. Mit Blick auf die Komponenten, die sich auf der Ebene der Services befinden, lässt sich diese folglich als organisationsweite und standardisierte Schnittstellen- und Kommunikationsschicht auffassen.

\subsubsection{Ebene der Prozesse}

Als weitere Schicht in dem SOA-Architekturmodell ist die Ebene der Prozesse eingebunden. Der Gegenstandsbereich dieser Schicht liegt darin, Services zu einem Gesamtprozess mit dem Ziel zu verknüpfen, die im Rahmen einer SOA angestrebte Automatisierung und/oder Flexibilisierung von Prozessen über Systemgrenzen hinaus zu erreichen. ${ }^{560}$ Zur Erfüllung dieses Ziels kommen Workflow Management-Systeme (WMS) zum Einsatz, deren Aufbau und grundsätzliche Funktionalität in Abschnitt 4.2.3.1 erläutert werden. Abschnitt 4.2.3.2 widmet sich den Kopplungsmechanismen, die bei der Verknüpfung von Services von Bedeutung sind. In Abschnitt 4.2.3.3 werden die Beziehungen zwischen den Komponenten der Ebene der Services erörtert.

\subsubsection{Worflow Management-Systeme}

Allgemein werden WMS genutzt, um Personen bei der Durchführung der Geschäftsprozesse zu unterstützen und den Ablauf der einzelnen Teilprozesse zu automatisieren. ${ }^{561}$ WMS werden mit dem Ziel betrieben, die Qualität der Geschäftsprozesse hinsichtlich der Integration von Prozessschritten und Informationsflüssen zu verbessern. ${ }^{562}$

Mit Blick auf den Einsatzzweck von WMS im Rahmen einer SOA besitzt diese Systemkategorie die Aufgabe, den Ablauf eines rollen- sowie applikationsübergreifenden Geschäftsprozesses durch den dynamischen Aufruf entspre-

Siehe hierzu Abschnitt 5.2.3.2.

560 Die Existenz einer Ablaufsteuerung ist nicht nur zur Ausführung von Berichtsprozessen erforderlich, sondern betrifft alle Unternehmungsprozesse, die eine abteilungsübergreifende Koordination erfordern. $\mathrm{Zu}$ diesen gehören beispielsweise der BalancedScorecard-Prozess sowie der Budgetierungsprozess. Vgl. Oehler (2006), S. 103.

561 Vgl. Deppisch/Oppitz (2004), S. 886.

562 Vgl. Deppisch/Oppitz (2004), S. 887. 
chender Dienste zu steuern. ${ }^{563}$ Dafür ist es notwendig, dass die gesamte Ablauflogik in einem ausführbaren Prozessmodell vorliegt. Ein Workflow lässt sich in diesem Zusammenhang als (Teil-)Prozess auffassen, der zur Ausführungszeit automatisiert durchlaufen werden kann. Jeder Service steht dabei für eine Prozessaktivität, was impliziert, dass jede Prozessaktivität aus untergeordneten Aktivitäten bestehen kann.

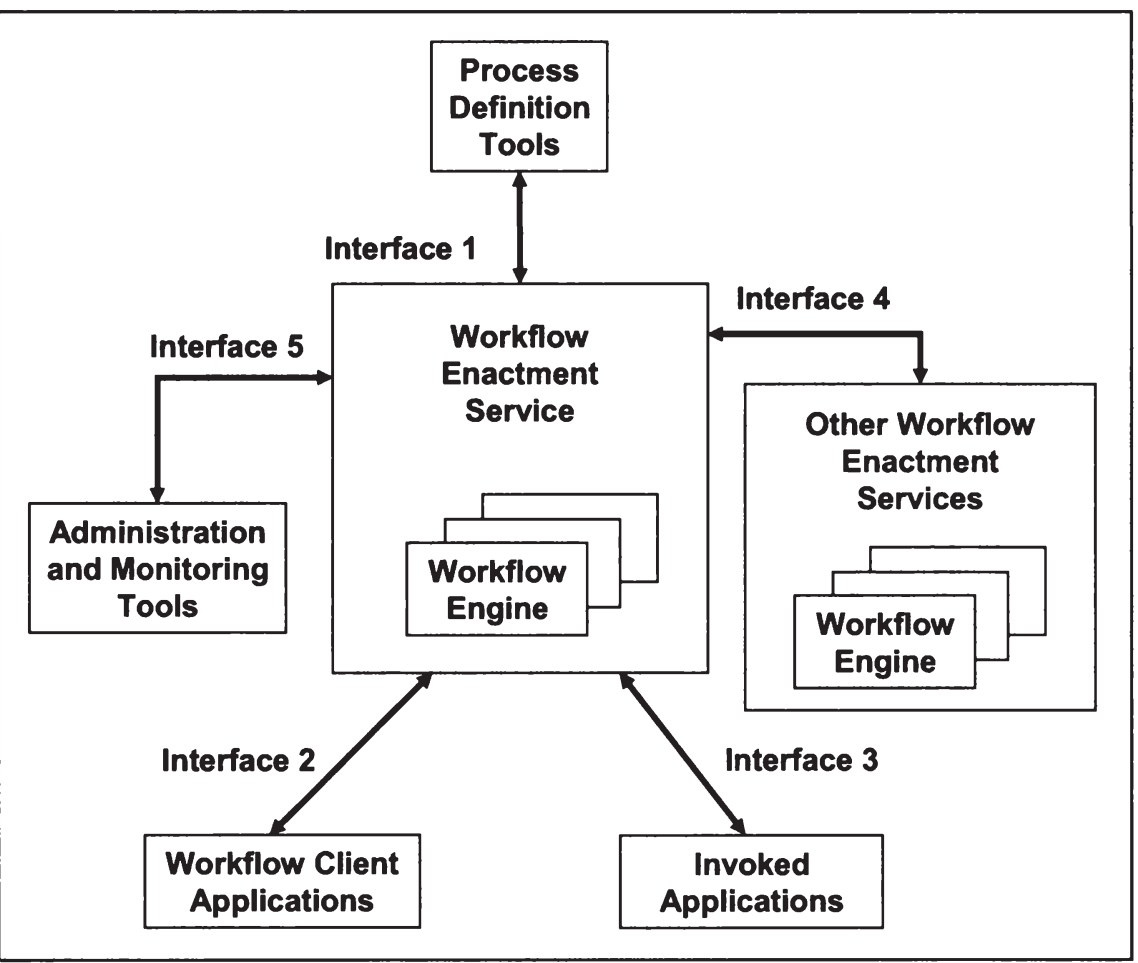

Abbildung 24: Bestandteile eines WMS564

Vor dem Hintergrund der Bedeutung von WMS für die Implementierung einer SOA werden im Folgenden die in Abbildung 24 illustrierten charakteristischen

563 Vgl. Hansen/Neumann (2005), S. 447. Diese Funktionalität wird in der von BERBNER ET AL. präsentierten Architektur einer Serviceorientierten Anwendung von einer ProxyKomponente übernommen. Vgl. Berbner et al. (2005), S. 273f.

564 In Anlehnung an Liebhart (2007), S. 89. 
Komponenten eines derartigen Systems vertieft. Die Vorstellung der Komponenten orientiert sich an dem Referenzmodell für WMS, das von der Workflow Management Coalition (WfMC) entwickelt wurde. ${ }^{565}$

Der Workflow Enactment Service (WES) stellt die zentrale Komponente eines WMS dar. Sie setzt sich aus den Workflow-Engines zusammen, deren Aufgabe es ist, die Workflows zu steuern, den Benutzern die durchzuführenden Aktivitäten zuzuordnen sowie externe Programme einzubinden. Ferner übernimmt der WES die Benutzerverwaltung. Weitere Aufgaben des WES umfassen die termingerechte Weiterleitung von Meldungen an andere Workflow-Systeme.

Als Process Definition Tools werden alle Werkzeuge bezeichnet, die zur Analyse, Modellierung, Beschreibung und Dokumentation der Geschäftsprozesse zum Einsatz kommen. Diese Werkzeuge lassen sich in ein WMS integrieren oder als eigenständige Tools betreiben. In zuletzt genanntem Fall erfolgt die Kommunikation mit dem WES über das Interface 1.

Als weitere Komponente sind die Workflow Client Applications (Interface 2) von Bedeutung. Workflow Client Applications lassen sich als Nachrichtendienst auffassen. Arbeitsschritte, die noch zur Bearbeitung anstehen, werden mit Hilfe der Workflow Client Applications in einer so genannten Worklist gespeichert. In ihr werden alle auszuführenden Arbeitsschritte eines Geschäftsprozesses und die an der Durchführung beteiligten Mitarbeiter dokumentiert. Nachdem die Tätigkeiten durch die Nutzer der Workflow Client Application abgearbeitet wurden, leitet diese die Ergebnisse an den WES weiter.

Invoked Applications als weitere Komponente eines WMS lassen sich über das Interface 3 an den WES anbinden. Hierbei handelt es sich um externe, eigenständige Applikationen wie Office-Tools und Datenbankanwendungen, die sich ohne eine Benutzerinteraktion aufrufen lassen, um die Abwicklung eines Arbeitsschrittes zu unterstützen. Die Anwendungen können auf verschiedenen Plattformen betrieben werden.

Das Interface 4 hat die Aufgabe, die Interoperabilität unterschiedlicher Informationen und Vorgangssteuerungen zwischen verschiedenen WMS zu gewährleisten. Mit Hilfe der Other Workflow Enactment Services lassen sich auf diese Weise laufende Prozesse synchronisieren. Ebenso können mit Hilfe dieser Komponente Prozesse verteilt auf mehreren Workflow-Servern ausgeführt werden.

Das Interface 5 dient zur Standardisierung und Verbindung der Administration and Monitoring Tools mit der Workflow-Engine. Mit Hilfe dieser Anwendungen lässt sich die Vorgangssteuerung unterstützen. Darüber hinaus stellen sie Informationen über den Ausführungsstatus und den Fortschritt einzelner Prozesse sowie den Zustand und die Auslastung der zugehörigen Instanzen zur Verfügung. Diese Informationen sind erforderlich, um die Kontrolldaten statis-

Vgl. Workflow Management Coalition (2009). 
tisch auswerten und Verbesserungsmöglichkeiten des Systems ableiten zu können.

\subsubsection{Kopplungsmechanismen zwischen Services}

Um die Ausführung applikations- und/oder unternehmungsübergreifender Geschäftsprozesse mit Hilfe von Services zu ermöglichen, ist es bedeutsam, die zum Einsatz kommenden Dienste zweckorientiert zu verbinden bzw. zu komponieren. Dabei wird mit dem Einsatz einer SOA das Ziel verfolgt, einzelne Services zu übergeordneten Diensten zu verknüpfen. ${ }^{566}$ Die übergeordneten Dienste wiederum lassen sich als Aktivitäten eines Serviceorientierten Geschäftsprozesses nutzen, wobei zur Service-Komposition zwei Ansätze denkbar sind: Die Dienste können orchestriert oder choreografiert werden. ${ }^{567} \mathrm{Da}$ im SOA-Umfeld in den meisten Fällen Web Services zum Einsatz kommen, orientieren sich die nachstehenden Ausführungen zu den beiden Ansätzen an dieser Technologie.

Die Orchestrierung bzw. Orchestration zeichnet sich dadurch aus, dass ein Web Service genutzt wird, um einen vollständigen Geschäftsprozess, der wiederum aus orchestrierten Services besteht, zu steuern. ${ }^{568}$ Der betreffende Web Service lässt sich mit Hilfe einer WSDL spezifizieren sowie unter Einsatz von SOAP aufrufen. Charakteristisch für eine Orchestrierung ist, dass eine ausführbare Geschäftslogik vorliegt, welche die Reihenfolge und alle Bedingungen zur Ausführung der verknüpften Services enthält. Für diesen Zweck muss eine Prozessbeschreibung erstellt und im Laufzeitsystem hinterlegt werden. Die Prozessbeschreibung enthält sämtliche Steuerungsinformationen, mit denen ein Service weitere Dienste aufruft, diese mit Daten versorgt und die Ergebnisse empfängt. Zur Koordination der Interaktionen der beteiligten Services kommt eine zentrale Steuerungseinheit zur Anwendung. Services, die, wie im linken und rechten Kasten der Abbildung 25 dargestellt, in einer bestimmten Ablaufreihenfolge festgelegt und für diesen Zweck parametrisiert sind, werden als orchestriert bezeichnet. 569

Charakteristisch für die Choreografie (Choreography) ist, dass kollaborative Geschäftsprozesse anhand der Interaktionen zwischen den verknüpften Services sowie der dadurch entstehenden Abhängigkeiten beschrieben werden. Im Gegensatz zur Orchestrierung von Services wird bei der Choreografie keine ausführbare Prozessdefinition vorgehalten. Lediglich die Interaktionen zwischen

566 Vgl. Winkler (2007), S. 258.

567 Vgl. vom Brocke (2007), S. 27f.

568 Der Bezeichnung „Orchestrierung“ liegt die Vorstellung zugrunde, dass der zentrale Service Aufgaben übernimmt, die sich mit den Aufgaben eines Orchesterdirigenten vergleichen lassen. Vgl. vom Brocke (2007), S. 27. 
den Services an den betreffenden Schnittstellen werden definiert. Folglich ist bei der Choreografie ausschließlich das „von außen“ sichtbare Verhalten der Services von Bedeutung. Eine zentrale Steuerungseinheit (wie bei der Orchestrierung) liegt bei der Choreografie nicht vor, so dass sich die choreografierten Services entsprechend der Prozessstruktur sequentiell selbst aufrufen. Für diesen Zweck müssen alle Steuerungs- und Nutzdaten zwischen den Services ausgetauscht werden. Nachdem der Auslöser der choreografierten Prozesskette den ersten Dienst ausführt, wird das Ergebnis des letzten Dienstes an den Auslöser übermittelt.

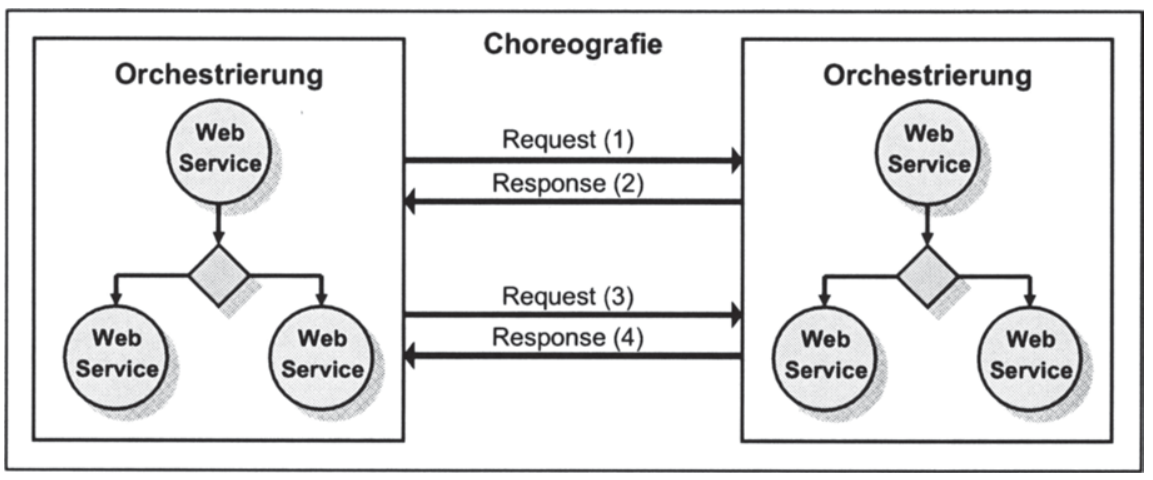

Abbildung 25: Zusammenhang zwischen Serviceorchestrierung und -choreografie

In der Abbildung 25 wird der Zusammenhang zwischen der Orchestrierung und der Choreografie von Services dargestellt. Festzuhalten ist, dass sich beide Ausprägungen zur Verknüpfung von Services in einer kombinierten Weise nutzen lassen. Während die Orchestrierung zur Steuerung innerbetrieblicher Prozesse verwendet werden kann, bietet die Choreografie Vorteile bei der zwischenbetrieblichen Ausführung von Geschäftsprozessen. ${ }^{570}$ Wie in der Abbildung 25 deutlich wird, lassen sich mit Hilfe der Choreografie verschiedene Orchestrierungen verbinden. In dem zugrunde liegenden Beispiel erfolgt nach einer Request-Nachricht (1 und 3) eine entsprechende Response-Nachricht (2 und 4). 
Zur Beschreibung und Automatisierung von Services werden aktuell insbesondere der BPEL-Standard und seine Nachfolger diskutiert. ${ }^{571}$ BPEL ist eine auf XML basierende Sprache zur Entwicklung von Geschäftsprozessen, die auf Basis von Web Services implementiert werden, und hat sich in diesem Bereich als De-facto-Standard etabliert. 572

Ein BPEL-Prozess umfasst die zeitliche und logische Abfolge von verschiedenen Web Service-Aufrufen und repräsentiert damit das informationstechnische Gegenstück zum fachlichen Prozessmodell.573 Mit IT-gestützten BPELWerkzeugen lässt sich der generierte BPEL-Prozess simulieren und die Zusammenstellung der prozesszugehörigen Services prüfen. Dadurch kann BPEL auch zur Qualitätssicherung bzw. -kontrolle einer SOA eingesetzt werden.574 Zudem lässt sich eine BPEL-Beschreibung digital archivieren und zu einem späteren Zeitpunkt wieder verwenden. Dadurch wird erreicht, dass die orchestrierten Services nicht erneut beschrieben werden müssen. ${ }^{575}$ Entsprechende Ressourcen können eingespart und für andere Aktivitäten eingesetzt werden.

Der Einsatz von BPEL zur Implementierung eines SOBP ist zu befürworten, da sich dieser Standard sowohl zur Orchestrierung als auch zur Choreografie von Web Services nutzen lässt. Demnach ist es möglich, diesen Standard zur Realisierung sowohl eines unternehmungsinternen als auch -übergreifenden Berichtsprozesses zu nutzen.

\subsubsection{Beziehungen zwischen den Komponenten der Prozessebene}

Wie Abbildung 26 zeigt, befindet sich die Ebene der Prozesse über der Ebene der Services. Die Ausführung der Prozessaktivitäten und damit der Services erfolgt anhand der Workflows, die mit Hilfe eines WMS implementiert und gesteuert werden. Damit sich die Services aufrufen lassen, muss das eingesetzte

571 Vgl. Modjo Kamneng (2007), S. 290. Bisher ist den verschiedenen Standardisierungsinitiativen nicht gelungen, einen einheitlichen Standard zur Orchestrierung von Web Services zu etablieren. Stattdessen konnten in der Vergangenheit eine Reihe von Standardisierungsvorschlägen präsentiert werden, die als so genannte „Flow-Languages“ in Konkurrenz zueinander stehen. Vgl. Herden et al. (2006), S. 70. Zu den bekanntesten Vertretern der Flow-Languages gehören die Business Process Execution Language (BPEL), Web Services Flow Language (WSFL), XLANG und Web Service Choreography Interface (WSCI). Kennzeichnend für diese Sprachen ist ihre Fähigkeit, im Gegensatz zu WSDL Zustände zu berücksichtigen.

572 Vgl. Andrews et al. (2003). Im Jahr 2002 wurde BPEL von IBM und MICROSOFT spezifiziert. Dabei stellt der Standard eine Vereinigung der kalkülbasierten Sprache XLANG von MICROSOFT mit den Konzepten der WSFL von IBM dar, indem BPEL die in WSDL beschriebenen Web Services zu einem Prozess bündelt. Vgl. Alves et al. (2006).

Vgl. Thomas/Leyking/Dreifus (2007), S. 43.

Vgl. Brodde (2006), S. 22.

Vgl. Modjo Kamneng (2007), S. 292. 
WMS auf die Middlewaredienste zugreifen, die wiederum die Ausführung der Services über die von ihnen bereitgestellte Schnittstelle ermöglichen.

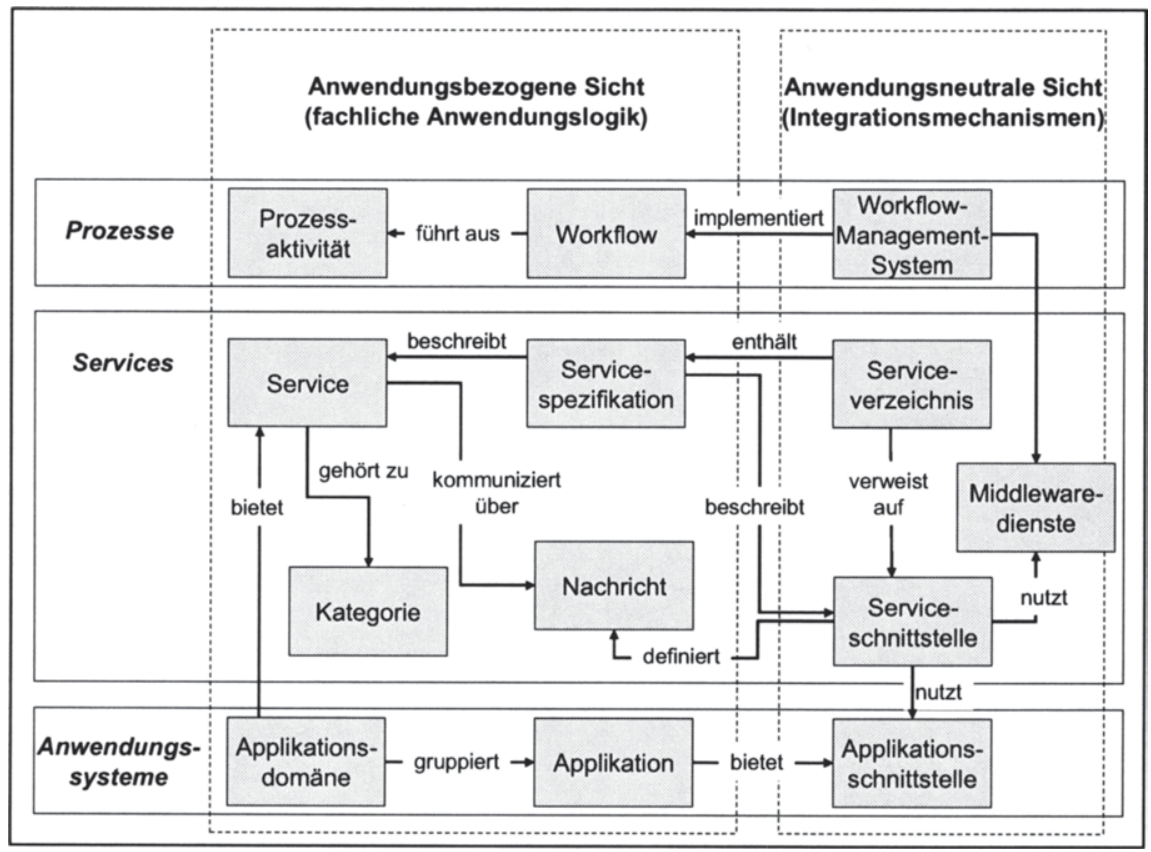

Abbildung 26: Erweiterung des SOA-Architekturmodells um die Prozessebene

\subsubsection{Ebene der Frontendsysteme}

Wie THOME bemerkt, hängt der Nutzen eines IS nicht von der technischen Überlegenheit sondern seiner Erreichbarkeit ab.576 Damit die jederzeitige Verfügbarkeit der Services gewährleistet werden kann, ist in das SOA-Architekturmodell eine Ebene einzubetten, die es den Anwendern ermöglicht, auf die Services zuzugreifen. Auf der obersten Schicht des SOA-Architekturmodells ist die Ebene der Frontendsysteme positioniert, die auch als Präsentations- und Interaktionsebene bezeichnet wird. Die Interaktionsebene bildet die Schnittstelle zwischen den Servicenutzern und den Services, die in einem Serviceverzeichnis 
abgelegt sind. Von den hier zum Einsatz kommenden Frontendsystemen sind zahlreiche Aufgaben zu erfüllen, die nun skizziert werden.

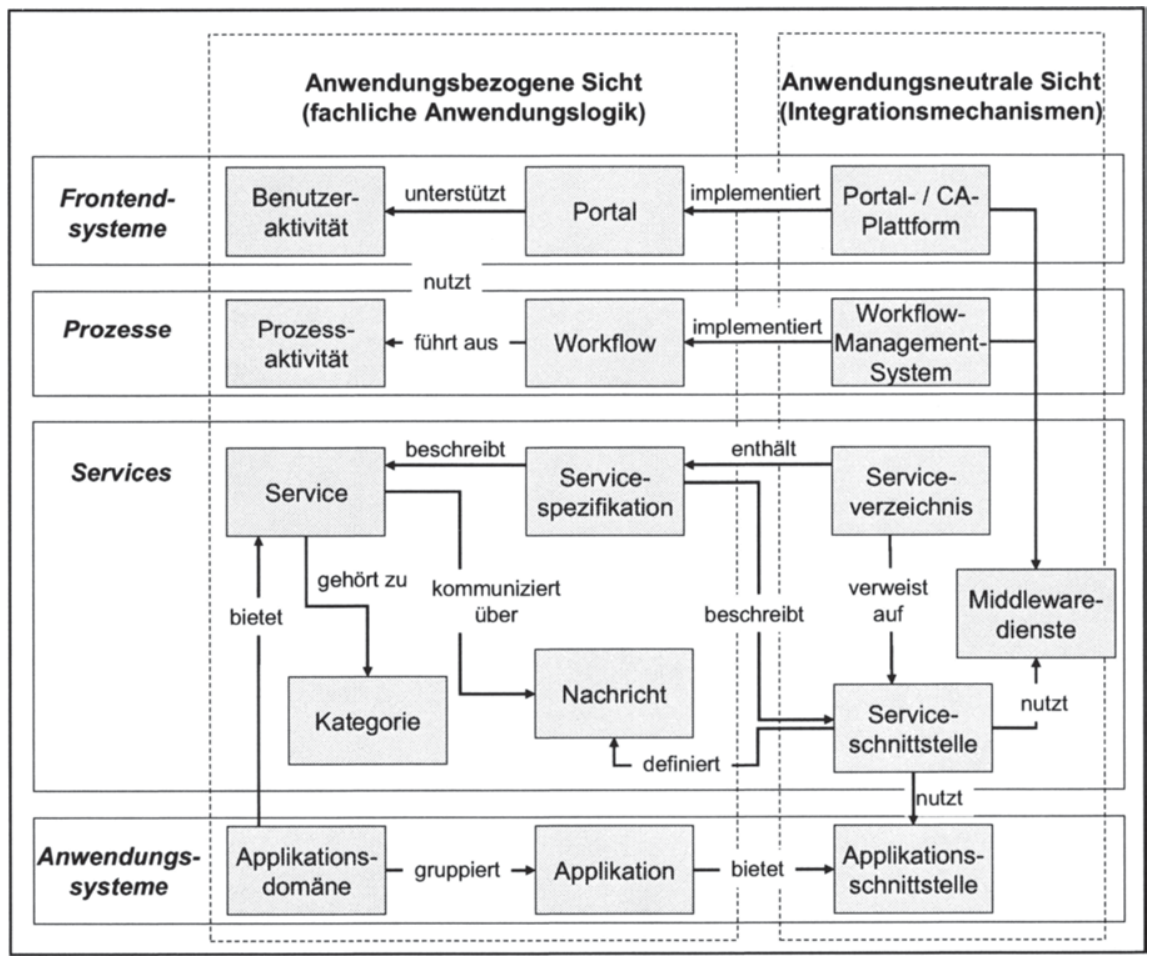

Abbildung 27: Erweiterung des SOA-Architekturmodells um die Ebene der Frontendsysteme

Eine Funktionalität der Frontendsystemen liegt darin, den Servicenutzern die relevanten Dienste über eine integrierte und individualisierte Sichtweise zur Verfügung zu stellen. Dies betrifft sowohl Dienste, die als Stand-AloneAnwendung einzelne Funktionen anbieten, als auch verknüpfte Services, die weitere Dienste im Rahmen einer Servicekette auslösen. In gleichem Maße, wie sich verschiedene Services aufrufen lassen müssen, ist von der Ebene der Frontendsysteme eine Schnittstelle bereitzustellen, damit sich auch unternehmungsexterne Dienste in den Servicebestand einbinden lassen. Darüber hinaus ist eine Zugriffsfunktion für alle Unternehmungsangehörigen einzurichten, die mit der Pflege und Wartung der Services, aber auch weiterer Komponenten einer SOA beauftragt sind. Ebenso sind entsprechende Mitarbeiter- bzw. Benutzerrollen für 
einen Zugriff auf die Dienste und alle weiteren technischen Komponenten einer SOA festzulegen. 577 Die für den Frontendzugriff relevanten Komponenten des SOA-Architekturmodells sind in der Abbildung 27 dargestellt.

Zur Ausführung Serviceorientierter Prozesse werden typischerweise Prozessportale eingesetzt. ${ }^{578}$ Ein Prozessportal ist eine Ausprägung eines Internetportals und stellt einen zielgruppenorientierten Zugriff zur Verfügung, um Nutzern die Ausführung von Benutzeraktivitäten und die Auslösung von Geschäftsprozessen zu ermöglichen. Damit stellen Prozessportale die Schnittstelle zwischen den Anwendungssystemen, den auszuführenden Prozessen und Nutzern dar. 579 Sie sind i. d. R. in Form einer Web-Anwendung realisiert.

Zur Implementierung von Prozessportalen lassen sich Portal- bzw. Composite-Application-Plattformen (CA-Plattformen) einsetzen. Diese stellen Entwicklungs- und Laufzeitdienste zur Verfügung, um desktopintegrierte Anwendungssysteme zu entwickeln. ${ }^{580}$ Wie in der Abbildung $27 \mathrm{zu}$ erkennen ist, nutzt eine Portalplattform dabei diverse Middlewaredienste.

\subsubsection{Eignung des SOA-Ebenenmodells für Serviceorientierte Berichtspro- zesse}

Mit Blick auf die in dieser Arbeit angestrebte Interoperabilität der im Rahmen von Berichtsanwendungen eingesetzten IS ist zu konstatieren, dass das vorliegende Architekturmodell den softwaretechnischen Gestaltungsrahmen liefert, um eine Anwendungsintegration kosteneffizient durchzuführen. Zur Herstellung der Anwendungsinteroperabilität haben die Services eine zentrale Bedeutung, da sie aus den Realisierungen der vorhandenen Anwendungen gewonnen werden können. Auf diese Weise lassen sich in einer SOA beispielsweise Services implementieren, die einen direkten Zugriff auf Daten ermöglichen, die in den Anwendungssystemen vorgehalten werden.

Die Aufteilung des Architekturmodells in die Ebenen der Anwendungssysteme, der Services, der Prozesse und der Frontendsysteme bietet das Potenzial, Geschäftsprozesse losgelöst von den sie unterstützenden Anwendungssystemen zu betrachten. ${ }^{581}$ Eine stärkere Trennung von Prozessmanagement und den Applikationen bringt in diesem Zusammenhang den Vorteil mit sich, den Fokus auf die Gestaltung bzw. auf das Redesign der zu unterstützenden Geschäftsprozesse richten zu können, ohne potenzielle technische Restriktionen beim Model-

577 Vgl. Heutschi/Legner/Österle (2006), S. 363.

578 Vgl. Hansen/Neumann (2005), S. 637.

579 Vgl. Grimm (2005), S. 17. Generell kommen Internet-Portale zum Einsatz, um ihren Nutzern einen direkten Zugang zum Informationsangebot sowie zu diversen Kommunikationsdiensten des Portal-Betreibers zu ermöglichen.

Vgl. Heutschi (2007), S. 191.

581 Vgl. Melzer et al. (2007), S. 32; Mattern (2003), S. 35. 
lierungsprozess in Erwägung ziehen zu müssen. ${ }^{582} \mathrm{Um}$ die Trennung zwischen der Logik der Geschäftsabläufe und den heterogenen und monolithischen Anwendungen einer Unternehmung zu überwinden, sind die aus dem Prozessmodell abgeleiteten Einzelschritte mit den bereits existierenden oder neu zu entwickelnden Services zu verknüpfen, um auf diese Weise eine betriebswirtschaftliche Funktion bzw. eine Geschäftsfunktion softwaretechnisch als Service zu implementieren. ${ }^{583}$ Die Inanspruchnahme von Funktionen erfolgt auf Basis eines hinterlegten Prozessmodells. Eine wichtige Aufgabe hat in dem entwickelten Architekturmodell der Einsatz eines WMS, das für die Ausführung und Koordination des hinterlegten Prozessmodells zuständig ist.

Im Ergebnis ist festzuhalten, dass das SOA-Architekturmodell alle technischen und logischen Komponenten aufführt, mit denen sich eine Automatisierung und/oder Flexibilisierung von SOBP durchführen lässt. Bei der Betrachtung der Ebenen und ihrer Komponenten muss konstatiert werden, dass eine semantische Verarbeitung von Informationen durch die bereitgestellten Komponenten nicht gewährleistet wird. ALT/HEUTSCHI/ÖSTERLE weisen in diesem Zusammenhang auf die Notwendigkeit hin, für die Kopplung von IS geeignete Standards einzusetzen, die beispielsweise die Berechnungsgrundlage zur Ermittlung eines Unternehmenswerts spezifizieren. ${ }^{584}$

Die im Rahmen des Einsatzes von Web Services thematisierten Standards SOAP, WSDL und UDDI reichen in diesem Zusammenhang allerdings nicht aus, da sie ausschließlich die technische Ebene fokussieren. ${ }^{585} \mathrm{Um}$ die in $\mathrm{Ab}$ schnitt 2.3 diskutierten Problemfelder in den Kernprozessen des Berichtswesens zu reduzieren, ist neben der Standardisierung von Prozessen eine weitere wichtige Aufgabe in der Standardisierung von Daten, die im Rahmen einer Serviceorientierten Anwendung verarbeitet werden sollen, zu erfüllen. Mit XBRL liegt die Empfehlung für einen derartigen Standard bereits vor. Da sich dieser explizit zur semantischen Beschreibung von Geschäfts- und Finanzinformationen, die bei der Ausführung von SOBP verarbeitet werden, einsetzen lässt, wird er in das hier fokussierte Architekturmodell eingebunden. Damit kann zudem der Forderung von BUSCH/DANGELMAIER entsprochen werden. Sie erklären, dass für eine softwaretechnische Implementierung von Diensten für einen unternehmungsübergreifenden Informationsaustausch stets geeignete Standards einzusetzen sind. 586

582 Siehe hierzu die Diskussion verschiedener Vorgehensweisen zur konzeptionellen Gestaltung von SOBP in Abschnitt 5.1.

583 Vgl. hierzu Heuser/Lacher/Perlmann (2007) S. 20.

$584 \mathrm{Vgl}$. Alt/Heutschi/Österle (2003), S. 68.

585 Zu diesen Standards vgl. Abschnitt 3.4.

586 Vgl. Busch/Dangelmaier (2002), S. 20. 


\subsection{Erweiterung des SOA-Architekturmodells um eine XBRL-Ebene}

Die vorliegenden Ausführungen haben zum Ziel, das bestehende Architekturmodell zu erweitern, um bei der Ausführung eines SOBP die Verarbeitung semantischer Geschäfts- und Finanzinformationen zu ermöglichen. Zu diesem Zweck wird das SOA-Ebenenmodell um eine Ebene erweitert. Diese hat den Einsatz der Extensible Business Reporting Language (XBRL) zum Gegenstand. Hierzu wird in Abschnitt 4.3.1 zunächst ein grundlegendes Verständnis von XBRL geschaffen. Den Taxonomien sowie Instanzen als zentrale Bausteine des XBRL-Standards widmet sich Abschnitt 4.3.2., während Abschnitt 4.3.3 die Einbindung von XBRL in das bestehende SOA-Architekturmodell fokussiert.

\subsubsection{Extensible Business Reporting Language (XBRL) als Enabler eines Serviceorientierten Berichtsprozesses}

Gegenstand des vorliegenden Abschnitts ist es, mit XBRL einen gegenwärtig weit verbreiteten und anerkannten IT-Standard vorzustellen, der auf der Extensible Markup Language (XML) aufbaut und als Enabler für einen SOBP zur Anwendung kommen kann. Zum Verständnis der Funktionsweise und Nutzungspotenziale von XBRL werden dazu vor allem die konzeptionellen und technischen Grundlagen dieses Standards erörtert. Mit Blick auf den XMLCharakter von XBRL thematisiert Abschnitt 4.3.1.1 zunächst die charakteristischen Eigenschaften von XML, die das Interesse an XBRL gefördert haben. Darauf aufbauend wird in Abschnitt 4.3.1.2 das für diese Arbeit relevante Verständnis von XBRL entwickelt und präzisiert.

\subsubsection{XML zur semantischen Beschreibung von Informationen}

Seit der Standardisierung von XML im Jahr 1997 wird der Einsatz dieser Sprache in verschiedenen betriebswirtschaftlichen Anwendungsbereichen diskutiert. 587 Als Teilmenge der Standard Generalized Markup Language (SGML) einem international anerkannten Standard zum Aufbau spezifischer Auszeich-

587 Einsatzmöglichkeiten von XML liegen etwa im Publishing und im ContentManagement, im elektronischen Handel und in der Unterstützung integrierter Geschäftsprozesse. Eine Übersicht zu diesen sowie weiteren Einsatzmöglichkeiten und zu ausgewählten Praxisbeispielen von XML findet sich bei Schöning (2003), S. 67ff. Für die Analyse der Auswirkungen von XML auf Electronic-Commerce-Anwendungen als eines der wichtigsten Einsatzfelder von XML vgl. Schinzer/Thome (1999), S. $212 \mathrm{ff}$. 
nungssprachen für elektronische Texte 588 - wurde XML vom WORLD WIDE Web Consortium (W3C) für den Austausch sowie die Ablage von Daten entwickelt. ${ }^{589}$ Bei XML handelt es sich um eine erweiterbare Auszeichnungssprache zur Spezifizierung beliebiger Daten- und Dokumentstrukturen. ${ }^{590}$ Charakteristisch für XML ist die Eigenschaft, die in einem XML-Dokument enthaltenen Informationen durch die Verwendung geeigneter Auszeichnungselemente, die mit Hilfe von Start- und Endtags definiert werden, nach semantischen Gesichtspunkten zu beschreiben. ${ }^{591}$ Bei der Verarbeitung der Informationen abstrahiert XML vollständig von konkreten Darstellungsformen.592 Die Struktur und das Layout werden von dem Inhalt des Dokuments folglich stringent separiert. 593 Diese Trennung hat zur Konsequenz, dass sich der Informationsgehalt eines XML-Dokuments unabhängig von den Formatierungsanweisungen verarbeiten lässt. 594

Mit Blick auf die webgestützte Darstellung von Informationen bietet der Sprachvorrat der HYPERTEXT MARKUP LANGUAGE (HTML) zwar die Möglichkeit, die zu publizierenden Informationen bedarfsgerecht und „optisch ansprechend" auf dem Bildschirm des Informationsempfängers zu visualisieren, ${ }^{595}$ eine inhaltliche Beschreibung der ausgezeichneten Daten findet dabei allerdings nicht statt, was als wesentlicher Nachteil von HTML anzusehen ist. Darüber hinaus ist eine automatisierte Übernahme von Daten, die in einem HTMLDokument abgelegt sind, nicht unproblematisch, weil HTML keine inhaltlichen Beschreibungskonstrukte vorhält, die eine unmittelbare Interpretation der dargestellten Inhalte zulassen. Vor diesem Hintergrund müssen HTML-Daten entweder in ein neues Dokument kopiert, direkt in ein Programm geladen oder neu eingegeben werden, bevor sie durch die IT weiterverarbeitet werden können. 596 Aufgrund der dabei durchzuführenden Anpassungs- bzw. Transformationsarbei-

Vgl. Schinzer/Thome (1999), S. 209. SGML wurde 1986 von der INTERNATIONAL ORGANISATION FOR STANDARDIZATION (ISO) als Standard verabschiedet (ISO 8879:1986). Zur Entwicklung von SGML sowie zum Verhältnis zwischen SGML und XML vgl. Eckstein/Eckstein (2004), S. 4f.; Schöning (2003), S. 2 f.

Vgl. Leser/Naumann (2007), S. 23.

Vgl. Fellmann/Thomas (2008), S. 972.

Vgl. Fellmann/Thomas (2008), S. 972.

Vgl. Eckstein/Eckstein (2004), S. 8f.

Vgl. Schwalm/Bange (2004), S. 7; Fellmann/Thomas (2008), S. 973.

Vgl. Baars (2005), S. 193. Für Präsentations- bzw. Formatierungszwecke kommen - wie auch bei der Anwendung von HTML - so genannte Style Sheets zum Einsatz, die in Form von einer Datei oder mehreren Dateien vorliegen können. Vgl. Meyer-Pries/ Gröner (2002), S. 45.

595 An dieser Stelle ist darauf hinzuweisen, dass es sich bei HTML um eine konkrete Ausprägung von SGML handelt, während XML eine Teilmenge von SGML darstellt. Vgl. hierzu Schinzer/Thome (1999), S. 209.

Vgl. Meyer-Pries/Gröner (2002), S. 45. 
ten geht eine derartige Vorgehensweise mit der Gefahr einher, eine schnelle, verlässliche und aussagekräftige Berichterstattung zu behindern. 597

Die Entwicklung von XML hatte das Ziel, eine Sprache zu schaffen, mit deren Hilfe sich die Inhalte eines Dokuments automatisiert verarbeiten lassen. Die angeführten Schwächen von HTML sollten folglich beseitigt werden, 598 wobei den so genannten Elementen als Kernbausteine der XML-Syntax eine fundamentale Rolle zukommt. ${ }^{599}$ Durch sie lassen sich die zu verarbeitenden Inhalte eindeutig spezifizieren, indem frei wählbare Elemente eingesetzt werden. ${ }^{600}$ Wie Abbildung 28 darstellt, erfolgt die Anordnung der Elemente dabei anhand einer hierarchischen Baumstruktur. ${ }^{601}$ Das in der Abbildung angeführte Beispiel zeigt in diesem Kontext die Elemente einiger Posten der Passivseite einer nach HGB erstellten Bilanz. Ausgehend von einem Wurzelelement mit der Bezeichnung Passivseite lassen sich weitere Unterelemente in einer verschachtelten Struktur anordnen, um die Werte der jeweiligen Bilanzposten der Passivseite zu beschreiben. 602

Die XML-Syntax schreibt den Einsatz derartiger Elemente zur semantischen Auszeichnung vor. Im Gegensatz zu HTML ist die exakte Bezeichnung der zu verwendenden Elemente nicht vorgegeben. Mit Blick auf die freie Wählbarkeit der Elemente lassen sich diese je nach Anwendungsbereich also flexibel definieren. ${ }^{603}$ Vor dem Hintergrund dieser Eigenschaft sowie der sämtlichen XMLDokumenten zugrunde liegenden hierarchischen Datenstruktur ermöglicht diese Sprache, dass die Inhalte nicht nur maschinell verarbeitet, sondern auch von den menschlichen Aufgabenträgern verstanden bzw. interpretiert werden können. Die in einem XML-Dokument beschriebenen Inhalte sind dabei nicht auf die semantische Auszeichnung strukturierter Inhalte beschränkt. In gleichem Maße lassen sich auch unstrukturierte und verschiedenartige multimediale Inhalte darstellen.

597 Vgl. hierzu die in Abschnitt 2.3 diskutierten Problemfelder für ein effektives und effizientes Reporting.

598 Zum Zusammenhang zwischen HTML und XML vgl. Kazakos/Schmidt/Tomczyk (2002), S. 8f.; Schinzer/Thome (1999), S. 208f. Zur Entstehung sowie zu den Entwurfszielen von XML vgl. Klettke/Meyer (2003), S. $21 \mathrm{f}$.

599 Vgl. Eckstein/Eckstein (2004), S. 20; Klettke/Meyer (2003), S. 24. Die Autoren liefern ferner eine Einführung in den syntaktischen Aufbau von XML-Dokumenten sowie die damit verbundene Verwendung der Elemente. Vgl. Eckstein/Eckstein (2004), S. 19ff.; Klettke/Meyer (2003), S. 24ff.

600 Vgl. Schöning (2003), S. 4f.

601 Vgl. Klettke/Meyer (2003), S. 24.

602 In Abbildung 28 wird deutlich, dass zur semantischen Auszeichnung des Inhalts - beispielsweise von 5.000.000 im Fall des gezeichneten Kapitals - ein Start- sowie ein Endtag des jeweiligen Elements verwendet werden.

603 Hierin liegt nach MARTENS ET AL. ein wichtiger Faktor für den Erfolg von XML. Vgl. Martens et al. (2006), S. 771. 


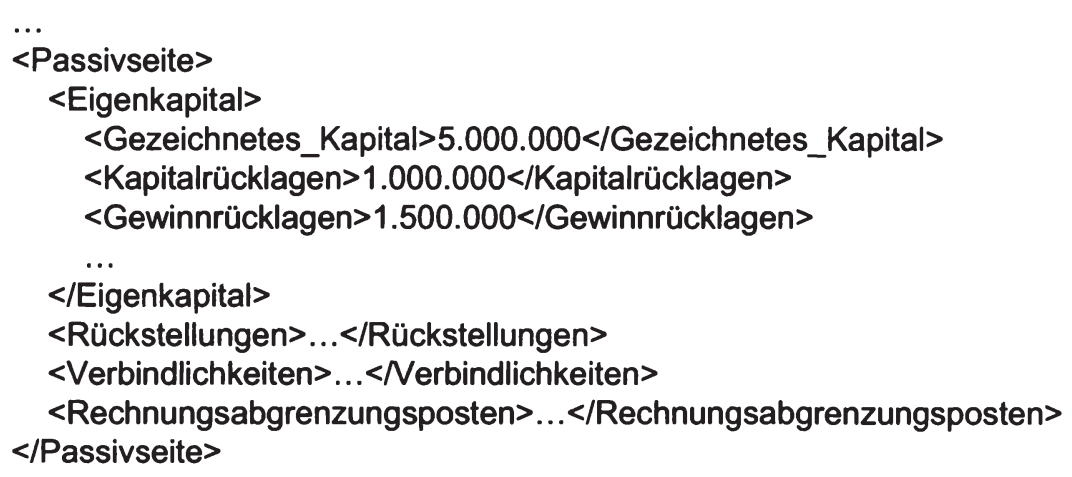

\section{Abbildung 28: Beispiel für ein XML-Dokument}

Zusammenfassend lässt sich festhalten, ${ }^{604}$ dass sich die Potenziale von XML vor allem beim Datenaustausch und bei persistenten Datenablagen entfalten.605 Als Schnittstellenstandard eröffnet XML damit diverse Einsatzmöglichkeiten, wobei der Standard insbesondere bei solchen Aufgaben zum Einsatz kommt, die eine web- und semantikgestützte sowie automatisierte Datenverarbeitung erfordern. Die Flexibilität und die Unabhängigkeit von bestimmten Anwendungsdomänen erweisen sich hier als besondere Stärken.

\subsubsection{Definition von XBRL}

Die (Weiter-)Entwicklung von XBRL in den vergangenen Jahren war von dem Ziel geleitet, sich der Stärken von XML für die Zwecke des Unternehmensreportings zu bedienen. ${ }^{606}$ Mit XBRL sollte ein Standard geschaffen werden, mit dessen Hilfe sich Geschäfts- und Finanzinformationen semantisch beschreiben sowie effizient und effektiv (weiter-)verarbeiten lassen. Um die zu verarbeitenden Geschäfts- und Finanzinformationen mit einer Bedeutung auszeichnen zu können, musste ein Vorrat an Elementen mit entsprechenden Beziehungsstrukturen festgelegt werden, die sich für diese Zwecke nutzen lassen.

Unter der Dachorganisation XBRL INTERNATIONAL entstanden in diesem Zusammenhang verschiedene Divisionen, welche die nationale sowie internationa-

604 Da die Nutzenpotenziale von XML in der Literatur hinreichend ausgearbeitet wurden, erfolgt an dieser Stelle nur eine kurze Zusammenfassung der wichtigsten Aspekte. Eine Vorstellung der Nutzenpotenziale von XML liefert z. B. Schöning (2003), S. $62 \mathrm{ff}$. Vgl. Gabriel/Röhrs (2003), S. 396; Leser/Naumann (2007), S. 23.

606 Zur Geschichte sowie zu den Entwicklungsschritten von XBRL vgl. DeFelice (2007), S. $30 \mathrm{f}$. 
le Etablierung von XBRL als Kommunikations- und Reportingstandard forcierten. Diese entsprechen meist Zusammenschlüssen von Software- und Beratungsunternehmungen, Forschungseinrichtungen und Anwenderunternehmungen, welche die (Weiter-)Entwicklung von XBRL aktiv vorantreiben. 607

Als XML-Dialekt verfügt XBRL über alle Eigenschaften, die sich aus der vom W3C propagierten Spezifizierung zur Meta-Sprache XML ergeben. ${ }^{608} \mathrm{Als}$ Synthese einiger ausgewählter Definitionsvorschläge lässt sich XBRL als frei verfügbarer, plattform- und systemunabhängiger (technologieunabhängiger) Open-Source-Kommunikationsstandard für die automatisierte und softwareunterstützte Erstellung, Verbreitung, Veröffentlichung und Auswertung von monetären und nicht monetären Geschäfts- und Finanzinformationen verstehen. $609 \mathrm{Da}$ XBRL als kostenfreier und offener Standard zur Verfügung steht, unterliegt die Spezifikation nicht der Marktmacht einer einzelnen Unternehmung.

\subsubsection{Bausteine des XBRL-Standards}

Die Standardisierungsempfehlung zu XBRL setzt sich aus zwei Bausteinen zusammen. Bei diesen handelt es sich zum einem um die XBRL-Taxonomien, zum anderen um die XBRL-Instanzen. ${ }^{610}$ Die Vorstellung beider Bestandteile ist Gegenstand der Abschnitte 4.3.2.1 und 4.3.2.2.

\subsubsection{XBRL-Taxonomie}

In einem allgemeinen Verständnis lässt sich eine Taxonomie als ein vorher festgelegtes, hierarchisches Schema definieren, das dazu dient, Informationen zu klassifizieren bzw. kategorisieren.611 Durch die Segmentierung sprachlicher Einheiten lässt sich auf diese Weise ein domänenspezifisches Sprachsystem anlegen, das für die Verarbeitung von Informationen der jeweiligen Anwendungsdomäne verwendet werden kann. ${ }^{612} \mathrm{Im}$ Kontext von XBRL kommen mit dem XBRL-Schema und den XBRL-Linkbases zwei Bausteine einer XBRLTaxonomie zum Einsatz, deren Grundlagen in den Abschnitten 4.3.2.1.1 und 4.3.2.1.2 vertieft werden.

Vgl. XBRL International (2009); Hodge/Kennedy/Maines (2004), S. 688.

608 Vgl. Eccles/Watson/Willis (2007), S. 207; Helmerich/Hümpfner/Scherer (2004), S. 623; Meall (2007), S. 73f.

609 Diese Definition basiert auf den Darstellungen von Baars (2005), S. 193; Felden (2006), S. 32; Meyer-Pries/Gröner (2002), S. 45; Pandrangi (2003); Schwalm/Bange (2004), S. 10.

610 Vgl. hierzu Hannon (2005), S. 58.

611 Vgl. Laudon/Laudon/Schoder (2006), S. 464

612 Vgl. hierzu Grothe/Gentsch (2000), S. 218. 


\subsection{XBRL-Schema als Normierungs- und Strukturvorschrift einer XBRL-Taxonomie}

Da sich XML durch eine hohe Flexibilität bzw. Erweiterbarkeit auszeichnet, muss definiert werden, welche Dokumentinhalte und -strukturen in dem jeweiligen Anwendungsbereich, in dem das Schema genutzt werden soll, zugelassen sind. ${ }^{613}$ Mit Hilfe von XML-Schema lassen sich alle Elemente sowie Attribute einer Anwendungsdomäne vollständig, systematisch und strukturiert spezifizieren. ${ }^{614}$ Die Attribute werden dabei verwendet, um den zulässigen Wertebereich der Elemente festzulegen.615 Die daraus resultierende Vorschrift wiederum liefert eine anwendungsspezifische Schablone bzw. Grammatik zur Erstellung und Validierung von so genannten Instanzdokumenten, deren Elemente, Attribute sowie Werteinträge den Vorgaben des jeweiligen XML-Schemas genügen müssen. ${ }^{616}$ Auf diese Weise lässt sich die syntaktische Korrektheit der XMLInstanzdokumente konsequent sicherstellen. ${ }^{617}$

Im Zusammenhang mit XBRL ist innerhalb eines XBRL-Schemas vorgegeben, welche Konzepte sich zur semantischen Auszeichnung der Geschäfts- und Finanzinformationen verwenden lassen. ${ }^{618}$ Die Konzepte werden durch die Elemente repräsentiert. Alternativ werden die Elemente auch als Items sowie Informationseinheiten bezeichnet. 619 Wie in der Abbildung 29 am Beispiel des Bilanzpostens Bruttoumsatz (Gross Profit) verdeutlicht ist, werden zur Beschreibung der Elemente verschiedene Attribute verwendet. Neben dem Elementnamen (name $=$ "GrossProfit") und einer ID (id="ifrs-gp_GrossProfit"), die zur

613 Vgl. Martens et al. (2006), S. 771.

614 Vgl. Schöning (2003), S. 33. Die Verabschiedung des XML-Schemas durch das W3C erfolgte im Mai 2001. Vgl. Klettke/Meyer (2003), S. 115. Eine Alternative zum Einsatz des XML-Schemas sind so genannte Document-Type-Definitions (DTD). Da DTD im Vergleich zu einem XML-Schema nur eingeschränkte Nutzungsmöglichkeiten haben, wird aktuell verstärkt das XML-Schema zur Gestaltung XML-basierter Anwendungen genutzt. Zu den Einschränkungen von DTD vgl. Martens et al. (2006), S. 771. Eine Übersicht über die im Vergleich zu DTD umfangreicheren Darstellungsmöglichkeiten des XML-Schemas liefern Klettke/Meyer (2003), S. $115 \mathrm{ff}$.

615 Vgl. Eckstein/Eckstein (2004), S. 83.

616 Eine Einführung in die grundlegenden Konzepte von XML-Schema präsentieren unter anderem ECKSTEIN/ECKSTEIN und KAZAKOS/SCHMIDT/TOMCZYK. Vgl. Eckstein/ Eckstein (2004), S. 83ff.; Kazakos/Schmidt/Tomczyk (2002), S. 39ff.

617 Vgl. Fellmann/Thomas (2008), S. 974. Syntaktisch korrekte XML-Dokumente werden auch als „wohlgeformt“ (well-formed) bezeichnet. Zu den Eigenschaften wohlgeformter XML-Dokumente vgl. z. B. Klettke/Meyer (2003), S. 38f. Gültige bzw. valide XMLDokumente genügen darüber hinaus Regeln, die die Nutzung von bestimmten Elementen und Attributen in einem XML-Dokument definieren. Derartige Regeln sind im XML-Schema spezifiziert. Vgl. Fellmann/Thomas (2008), S. 974.

Vgl. Baars (2005), S. 193; Kranich/Schmitz (2003), S. 77f.

619 Vgl. Kranich/Schmitz (2003), S. 78. 
eindeutigen Kennzeichnung dieses Elements dienen, lassen sich weitere Attribute nutzen. Die vorlaufende Bezeichnung "ifrs-gp" deutet darauf hin, dass das GrossProfit-Element im Rahmen der IFRS-General-Purpose-Taxonomie spezifiziert ist. 620

\begin{tabular}{|l|l}
\hline \multicolumn{1}{|c|}{ Attribut } & \multicolumn{1}{c}{ Wert } \\
\hline name & "GrossProfit" \\
\hline id & "GrossProfit" \\
\hline type & "monetaryltemType" \\
\hline periodType & "duration" \\
\hline balance & "credit" \\
\hline abstract & "false" \\
\hline nillable & "true"
\end{tabular}

\section{Abbildung 29: Ausgewăhlte Attribute eines XBRL-Taxonomieelements}

Mit Hilfe des type-Attributs wird der Datentyp für das jeweilige Element festgelegt. Bei dem hier betrachteten Taxonomieelement kommt mit "monetaryltemType" ein Datentyp für Währungsbeträge zum Einsatz. Das Attribut mit der Bezeichnung periodType gibt Auskunft darüber, ob es sich bei dem betrachteten Element um eine Strom- oder Bestandsgröße handelt, während das balanceAttribut informiert, ob das jeweilige Element auf der Aktivseite ("debit") oder Passivseite ("credit") steht. Hat das abstract-Attribut eines XBRL-Elements den Wert "false", kann es instanziert werden.621 Mit Hilfe des nillable-Attributs lässt sich festlegen, ob das Element Nullwerte aufnehmen kann. ${ }^{622}$ Ist diese Eigenschaft - wie in dem vorliegenden Fall - erfüllt, weist das Attribut einen positiven Wert ("true") auf.

620 Vgl. hierzu IASB (2005).

621 Vgl. hierzu Eckstein/Eckstein (2004), S. 153.

622 Vgl. hierzu Eckstein/Eckstein (2004), S. 136. 


\subsection{Beziehungstypen einer Taxonomie}

Zur Abbildung einer konsistenten Sicht auf die Geschäftszahlen einer Unternehmung ist es notwendig, die in einer Taxonomie definierten Elemente miteinander zu verknüpfen, um auf diese Weise Zusammenhänge abzubilden. So muss beispielsweise definiert sein, dass sich eine Bilanz aus einer Aktiv- und Passivseite zusammensetzt, welche wiederum ergänzende Bilanzpositionen enthalten. Zur Beschreibung der jeweiligen Beziehungsstrukturen stehen dem Entwickler einer XBRL-basierten Anwendung mit den Definition Links (Definition), Calculation Links (Berechnung), Presentation Links (Darstellung), Label Links (Bezeichnung) und Reference Links (Referenz) fünf Beziehungstypen zur Verfügung. 623 Anstelle des Terminus „Links“ ist auch die Bezeichnung „Linkbase“ gebräuchlich. Linkbases lassen sich als separate XML-Dokumente auffassen, ${ }^{624}$ die alle Anweisungen des jeweiligen Beziehungstyps beinhalten.625 Die fünf Beziehungstypen gliedern sich in zwei Beziehungsklassen - Relation Links und Documentation Links.

Die Beziehungsklasse Relation Links umfasst alle Beziehungstypen, die innerhalb einer Taxonomie zwischen den darin enthaltenen Elementen auftreten. Hierzu gehören die Beziehungstypen Definition Links, Calculation Links sowie Presentation Links. Der Beziehungstyp Definition Links dient dazu, die inhaltliche Zuordnung der Elemente in einer Taxonomie in Form hierarchischer Beziehungsstrukturen festzulegen.626 Auf diese Weise ist beispielsweise definiert, dass die Bilanzpositionen Vorräte und Kassenbestand zum Umlaufvermögen gehören. Calculation Links werden zur rechentechnischen Abbildung der Elementbeziehungen eingesetzt. ${ }^{627}$ Im Falle einer additiven Verknüpfung lassen sich die Werte untergeordneter Bilanzpositionen durch den Einsatz dieses Beziehungstyps zu einer übergeordneten Bilanzposition addieren. Alternativ können untergeordnete Elemente voneinander abgezogen werden, um ein neues übergeordnetes Ergebnis zu generieren. In der Kategorie der Presentation Links als letzte Ausprägung einer Relation Links-Beziehungsklasse wird festgelegt, in welcher Reihenfolge die einzelnen XBRL-Elemente angezeigt werden sollen. ${ }^{628}$

Vgl. Felden (2006), S. 33; Gehra/Hess (2004), S. 403; Kranich/Schmitz (2003), S. 78.

624 Zur Definition der verschiedenen Link-Arten kommt die XML-Linking-Technologie (XLink) zum Einsatz. Sie wird eingesetzt, um Beziehungen zwischen Informationseinheiten sowie Referenzen zu externern Ressourcen zu spezifizieren. Vgl. Felden (2006), S. 33 .

Vgl. Ramin/Kesselmeyer/Ott (2006a), S. 183.

Vgl. Baars (2005), S. 193.

Vgl. Baars (2005), S. 193.

Vgl. Ramin/Kesselmeyer/Ott (2006b), S. 12. 
Bei diesem Beziehungstyp geht es folglich um die Strukturierung der Elemente nach logischen Gesichtspunkten. ${ }^{629}$

Die Beziehungsklassen Label Links und Reference Links, die zur Beziehungsklasse der Documentation Linkbases gehören, verfolgen das Ziel, die Bestandteile einer Taxonomie mit zusätzlichen Informationen anzureichern, um damit die Aussagekraft der Geschäfts- und Finanzinformationen zu steigern. ${ }^{630}$ Mit Hilfe der Label Links lassen sich den Konzepten einer Taxonomie Bezeichnungen in verschiedenen Sprachen zuweisen. ${ }^{631}$ Auf diesem Weg lässt sich eine Mehrsprachigkeit abbilden, so dass für den Bilanzposten Umlaufvermögen das Pendant "Current Assets", "Actifs Circulants" oder "Activo Circulante" in der jeweiligen englischen, französischen und italienischen Landessprache benutzt werden kann. Die Beziehungsklasse Reference Links kommt zur Anwendung, um z. B. Verweise auf eine Rechtsquelle als gesetzliche Grundlage oder auf eine maßgebende Literatur für ein XBRL-Element anzulegen. Entsprechend kann ein besseres Verständnis für das verwendete Konzept erzielt werden.

\subsubsection{Taxonomiekategorien von XBRL}

Je nach Informationsbedarf des Empfängers hinsichtlich des Detaillierungsgrads der zu verarbeitenden Inhalte einer XBRL-Instanz lassen sich mit XBRL Global Ledger (XBRL GL) und XBRL Financial Reporting (XBRL FR) zwei Taxonomiekategorien einsetzen. Beide Taxonomiekategorien werden in den Abschnitten 4.3.2.2.1 und 4.3.2.2.2 vorgestellt.

\subsection{XBRL Financial Reporting}

Die XBRL FR-Taxonomien wurden mit dem Ziel entwickelt, den Austausch aggregierter bzw. konsolidierter Geschäfts- und Finanzinformationen, wie sie beispielsweise in Form eines Jahresabschlusses veröffentlicht werden, zu unterstützen. In den vergangenen Jahren ist es der Dachorganisation XBRL INTERNATIONAL gelungen, eine Reihe von XBRL FR-Taxonomien zu erstellen und zur freien Nutzung anzubieten. 632

Dem Anwender stehen Taxonomien zur Verfügung, in denen gegenwärtig etablierte Rechnungslegungsstandards wie beispielsweise IAS/IFRS, US-GAAP oder HGB abgebildet sind. Für jeden Bestandteil dieser Standards ist dabei typischerweise eine separate Taxonomie publiziert, wobei eine vollständige HGB-

629 Vgl. Felden (2006), S. 33.

630 Vgl. Baars (2005), S. 193.

$631 \mathrm{Vgl}$. Ramin/Kesselmeyer/Ott (2006b), S. 12.

632 Eine Übersicht zu den vorhandenen Taxonomien ist unter XBRL INTERNATIONAL abrufbar. Vgl. XBRL International (2009). 
Abschluss-Taxonomie z. B. die Bereiche „Allgemeine Informationen“, „Bilanz“, „GuV-Rechnung“, „Ergebnisverwendung“, „Eigenkapitalspiegel“, „,Kapitalflussrechnung“, „Anlagespiegel“", „Segmentberichterstattung“, „Liste des Anteilsbesitzes“, „,sonstige Notes“, „Lagebericht" sowie „Einladung/Topics der Hauptversammlung " beinhaltet. ${ }^{633}$

Neben Taxonomien zur Abbildung der landesspezifischen Rechnungslegungsstandards liegen darüber hinaus Taxonomien vor, die sich innerhalb eines Rechnungslegungsstandards auf die Weiterverarbeitung von Geschäfts- und Finanzinformationen in spezifischen Branchen beziehen. ${ }^{634}$ Exemplarisch sind in diesem Zusammenhang die „Commercial and Industrial-Taxonomie" sowie die „Banking and Savings-Taxonomie“ zu nennen, die im ersten Fall für die Finanzberichterstattung von Handels- und Industrieunternehmungen, im zweiten Fall für das Reporting von Unternehmungen aus dem Bankensektor eingesetzt werden können. Während die Taxonomien zu IAS/IFRS sowie US-GAAP einen derartigen Branchenbezug aufweisen, liegt eine dementsprechende Ausrichtung bei der aktuell vorhandenen HGB-Taxonomie nicht vor.

\subsection{XBRL Global Ledger}

Im Vergleich zu den XBRL FR-Taxonomien fokussiert XBRL GL nicht die Verarbeitung konsolidierter bzw. aggregierter Daten, die beispielsweise in einer Bilanz enthalten sind. XBRL GL lässt sich einsetzen, um Geschäfts- und Finanzdaten semantisch zu verarbeiten, die in einer geringeren Verdichtungsstufe vorliegen und damit als Grundlage für die Erstellung eines Jahresabschlusses dienen. ${ }^{635}$ Gegenstand des Datenaustauschprozesses sowie der Datenspeicherung unter Einsatz von XBRL GL sind folglich alle Daten, die in den Buchhaltungs- und Kostenrechnungssystemen einer Unternehmung verarbeitet werden.636 Unter Einsatz der XBRL GL-Taxonomie wird angestrebt, alle Hauptbucheinträge und entsprechenden Konten zu erfassen.637 Das Anwendungsfeld ist hierbei nicht auf finanzielle Daten beschränkt, sondern kann auch nicht finanzielle Daten umfassen. 638

Im Gegensatz zu den XBRL FR-Taxonomien sind bei der XBRL GLTaxonomie keine Elemente für einen bestimmten Rechnungslegungsstandard oder Bestandteil des Jahresabschlusses vorgegeben. ${ }^{639}$ Stattdessen liefert XBRL

Vgl. Meyer-Pries/Gröner (2002), S. 47f.

635 Vgl. Kranich/Schmitz (2003), S. 79; Meyer-Pries/Gröner (2002), S. 45; Garbellotto (2006a), S. 59.

636 Vgl. Ramin/Kesselmeyer/Ott (2006a), S. 183.

637 Vgl. Kranich/Schmitz (2003), S. 79.

$638 \mathrm{Vgl}$. Garbellotto (2006a), S. 59.

$639 \mathrm{Vgl}$. Ramin/Kesselmeyer/Ott (2006a), S. 181. 
GL den Rahmen, wie Buchungen sowie Transaktionsdaten semantisch auszuzeichnen und für einen Datenaustausch auf Basis von XBRL vorzubereiten sind. Auf diese Weise können diese Daten unabhängig von einem Rechnungslegungsstandard zwischen unternehmungsinternen und -externen XBRLkompatiblen IS ausgetauscht sowie gespeichert werden. ${ }^{640}$ Darüber hinaus lassen sich in den semantisch ausgezeichneten Buchungen über die Verwendung zusätzlicher Datenfelder Querverweise auf eine übergeordnete Bilanzposition herstellen, die wiederum als Element in einer XBRL FR-Taxonomie definiert ist. 641

Die XBRL GL-Taxonomie setzt sich aus einem Basismodul, das als Core (COR) bezeichnet wird, und vier Erweiterungen zusammen, mit deren Hilfe sich die Buchungs- bzw. Transaktionsdaten mit ergänzenden Informationen anreichern lassen. ${ }^{642}$

Wie in Abbildung 30 erkennbar ist, liegt dem Einsatz von XBRL GL die Prämisse zugrunde, dass alle zum Austausch vorgesehenen Daten in einem Buchungsprotokoll abgelegt sind. Dieses ist im jeweiligen Buchhaltungssystem vorzuhalten. ${ }^{643}$ Für den Austausch eines derartigen Buchungsprotokolls liefert das Basismodul COR von XBRL GL alle notwendigen Informationen. Alle in einem derartigen Protokoll abgelegten Daten werden als eine Gruppe von Buchungen aufgefasst. Diese Daten werden in der Sprache von XBRL GL ,accoutingEntries" genannt und bestehen aus einer oder mehreren Buchungssätzen (,entryHeader"), die sich wiederum aus einer oder mehrerer Buchungen zusammensetzen (,entryDetail“). ${ }^{644}$

$640 \mathrm{Vgl.} \mathrm{Ramin/Kesselmeyer/Ott} \mathrm{(2006a),} \mathrm{S.} 183$.

641 Vgl. Kranich/Schmitz (2003), S. 79; Garbellotto (2006a), S. 59. Die Verknüpfung von XBRL GL und XBRL FR lässt sich mit Hilfe der Datenfelder xbrlTaxonomy sowie xbrlElement durchführen. Vgl. Ramin/Kesselmeyer/Ott (2006a), S. 183.

642 Bei den Erweiterungen von COR handelt es sich um Module, welche die Bezeichnung Advanced Business Concepts (BUS), MultiCurrency (MUC), Concepts for the US, UK, etc. (USK) und Tax Audit File (TAF) tragen. Das BUS-Modul liefert Datenfelder, mit denen der Organisationsaufbau einer Unternehmung sowie weitere messbare Daten beschrieben werden können. In diesem Kontext lassen sich die Elemente einerseits zur Eingabe von Detaildaten z. B. im Anlagevermögen oder Vorratsvermögen nutzen. Andererseits können auch Kennzahlen wie beispielsweise Key-Performance-Indikatoren semantisch ausgezeichnet werden. Das MUC-Modul ergänzt das Basismodul um Elemente zur Währungsumrechnung. Das USK-Modul enthält Datenfelder, die tendenziell bei angelsächsisch geprägten Buchführungssystemen zum Einsatz kommen wie etwa der Aufriss der Debitorenkonten. Die Elemente aus dem TAF-Modul ermöglichen hingegen eine steuerliche Betriebsprüfung.

643 Vgl. Ramin/Kesselmeyer/Ott (2006a), S. 184.

644 Die Elemente des COR-Basismoduls liefern zu den einzelnen Buchungen Angaben zu den Buchungskonten (account), zu der gebuchten Menge (amount) sowie zu der Buchungszeit (postingDate). 


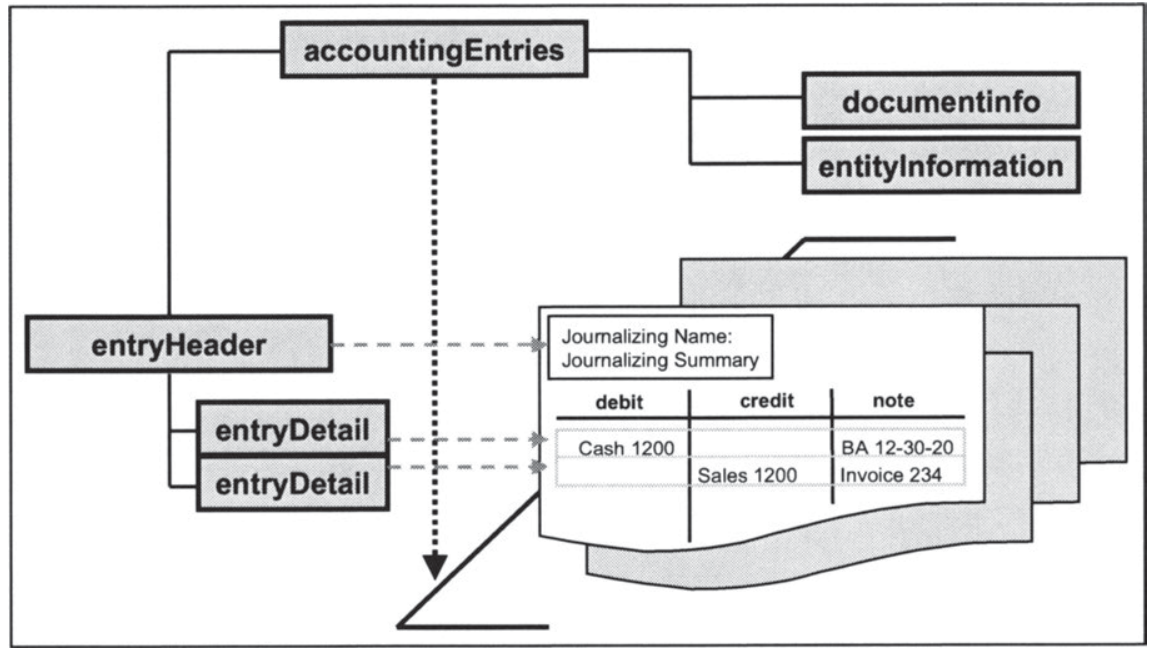

Abbildung 30: Aufbau von XBRL GL 645

Neben den Buchungssätzen lassen sich in den ,accountingEntries“ auch Summen- und Saldenlisten (balance), Buchungsjournale (journal) oder Saldenlisten einzelner Konten (ledger) abbilden. Darüber hinaus können auch weitere Informationen eingefügt werden, die indirekt aus der Buchhaltung stammen bzw. dem Hauptbuch vorgelagert sind. ${ }^{646} \mathrm{Zu}$ diesen Informationen gehören z. B. Offene-Posten-Listen, Vorratsinventarlisten oder der Anlagespiegel.

\subsubsection{Werttragende Instanzdokumente}

Während Taxonomien die inhaltlichen sowie strukturellen Vorgaben zur Verwendung von Konzepten des betrieblichen Berichtswesens enthalten, besitzen XBRL-Instanzdokumente die Aufgabe, die konkreten Berichtsdaten im XMLFormat vorzuhalten. ${ }^{647}$ Vor diesem Hintergrund lassen sich XBRL-Instanzen als werttragende Ausprägungen einer XBRL-Taxonomie bezeichnen.648 Die XBRL-Taxonomie dient dabei als Referenz bzw. Grammatik für das Instanzdokument. Dies hat zur Folge, dass eine valide XBRL-Instanz, die eine bestimmte XBRL-Taxonomie referenziert, die in der Taxonomie normierten Elemente, die

645 In Anlehnung an Ramin/Kesselmeyer/Ott (2006a), S. 184.

646 Vgl. Ramin/Kesselmeyer/Ott (2006a), S. 184.

647 Vgl. Baars (2005), S. 193; Pandrangi (2003).

648 MEYER-PRIES/GRÖNER sprechen in diesem Zusammenhang auch von einem „elektronischen“" Report. Vgl. Meyer-Pries/Gröner (2002), S. 49. 
Attribute ebenso wie die Beziehungen zwischen den Elementen enthalten muss. ${ }^{649}$

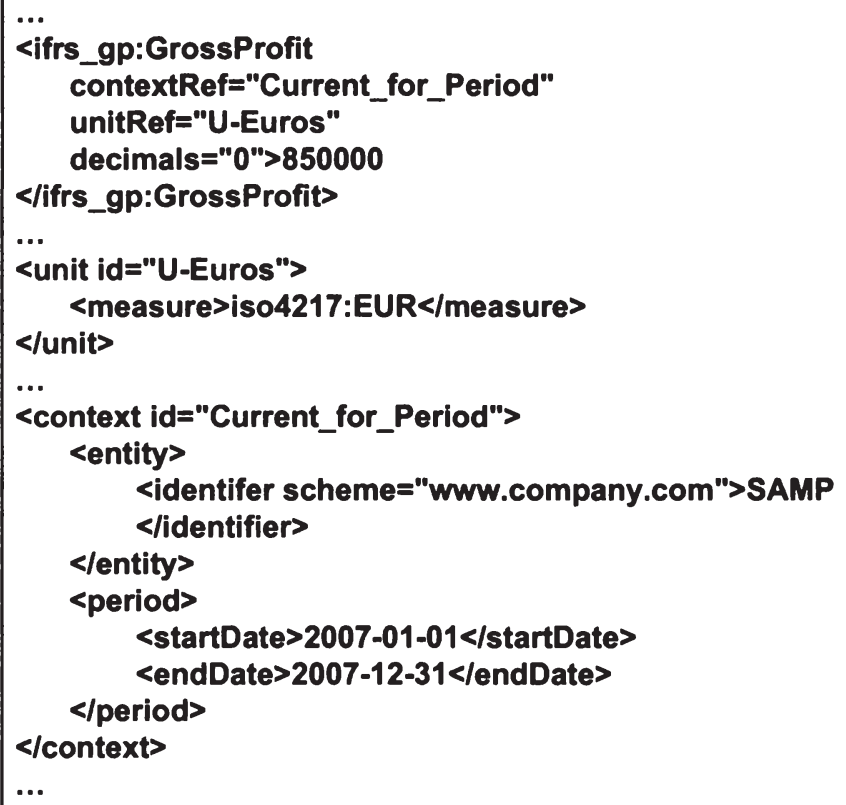

\section{Abbildung 31: Beispiel eines XBRL-Instanzdokuments}

Abbildung 31 stellt den Aufbau sowie einen Auszug des möglichen Inhalts eines XBRL-Instanzdokuments dar. Wie aus der Abbildung hervorgeht, enthält das Instanzdokument eine Reihe von Elementen, Attributen und Werten. Neben der Bezeichnung für das verwendete Taxonomieelement (GrossProfit) sind weitere Attribute zur Konkretisierung der Inhalte dieses Elements abgelegt.

Im gewählten Beispiel enthält das Instanzdokument einen Eintrag von 850.000 , der für die Höhe des Bruttogewinns steht. Aus dem Präfix "ifrs_gp" geht hervor, dass das GrossProfit-Element zu einem Namensraum für Schemaelemente gehört, die in der IFRS-General-Purpose-Taxonomie spezifiziert 
sind. ${ }^{650}$ Die Attribute unitref und contextref repräsentieren Verweise auf das unit- und context-Element, die wiederum weitere Unterelemente, Eigenschaften und Inhalte umfassen. Während das unit-Element eine Angabe über die zum Einsatz kommende Währung liefert, informiert das context-Element über die Gültigkeit dieses Eintrags, was über ein diesbezügliches Anfangs- (startdateElement) und Enddatum (enddate-Element) dargestellt wird. Ferner ist aus dem illustrierten Beispiel ableitbar, dass sich der Bruttogewinn auf das Geschäftsjahr 2007 bezieht. Dabei handelt es sich um einen Eurobetrag, welcher keine Dezimalstellen zulässt. Letztere Angabe wird über das decimals-Attribut bestimmt.

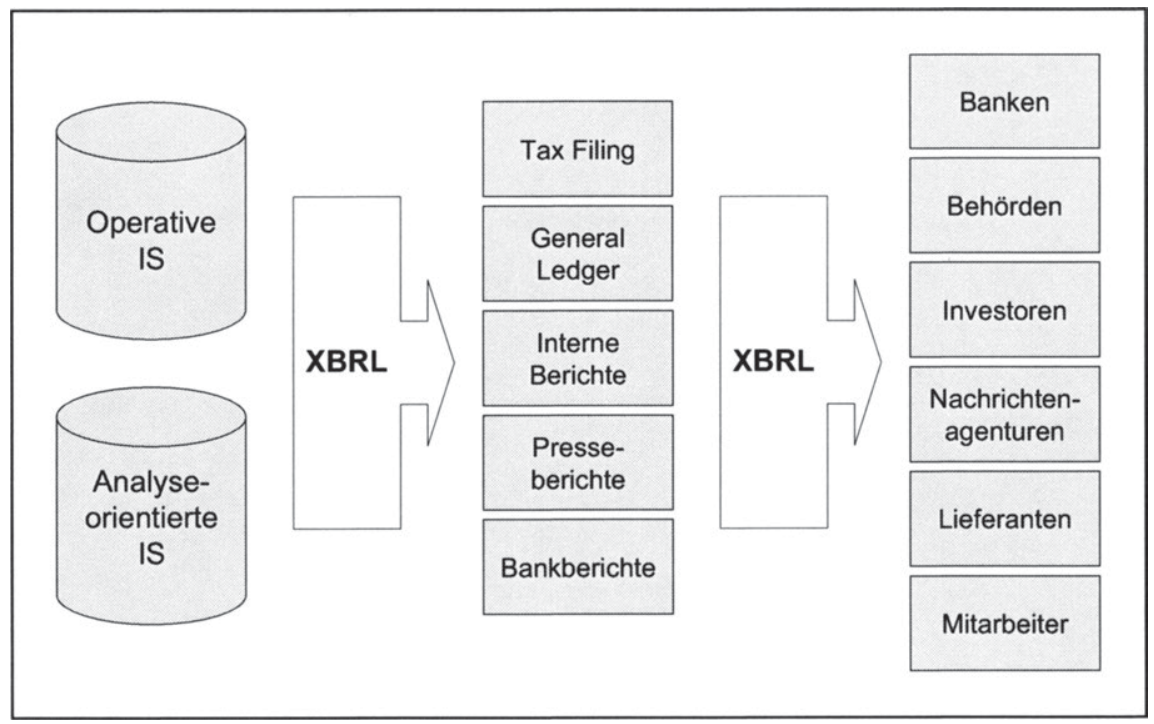

Abbildung 32: Nutzungsmöglichen von XBRL 651

Es lässt sich zusammenfassen, dass XBRL sowohl zur Verarbeitung operativer Berichtsdaten als auch zur Verarbeitung von Daten eingesetzt werden kann, die in Analyseorientierten IS (wie z. B. in DWH-Anwendungen) vorgehalten werden. Wie Abbildung 32 zeigt, ergeben sich hinsichtlich der Anwendungsmöglichkeiten von XBRL zahlreiche Einsatzfelder, wobei als nationale und internationale Adressaten eines Reportings auf Basis von XBRL die Shareholder einer

650 Vgl. hierzu IASB (2005).

651 In Anlehnung an Oehler (2006), S. 109. 
Unternehmung genauso in Frage kommen, wie ihre Stakeholder. ${ }^{652}$ Dabei bringt XBRL die Möglichkeit mit sich, dass sich der Standard sowohl für die Zwecke des internen als auch externen Reportings einsetzen lässt. ${ }^{653}$

\subsubsection{Einbindung der XBRL-Konzepte in das SOA-Ebenenmodell}

Abbildung 33 verdeutlicht das vollständige Architekturmodell, das sich zur konzeptionellen Gestaltung eines SOBP nutzen lässt. Festzuhalten ist, dass die XBRL-Ebene zwischen der Ebene der Anwendungssysteme und der Serviceebene positioniert ist. Als Architekturkomponenten der XBRL-Ebene kommen die XBRL-Taxonomien und die XBRL-Instanzen zum Einsatz.

Wie aus der Abbildung deutlich wird, dienen die XBRL-Taxonomien als Referenz für die XBRL-Instanzen. Mit Blick auf die kombinierte Verwendung von XBRL und Services ergeben sich zwei Nutzungsmöglichkeiten. Zum einen können Services direkt auf die Daten eines XBRL-Instanzdokuments zugreifen. In diesem Fall kommen XBRL-Instanzen als Informationsquelle für die Services zum Einsatz. Zum anderen ist es möglich, dass Dienste direkt die Daten der Anwendungssysteme verarbeiten. Wie beim Zugriff eines Services auf ein XBRL-Instanzdokument dienen die XBRL-Taxonomien in dieser Konstellation als Validierungsinstrument, das sicherstellt, dass nur Geschäfts- und Finanzinformationen verarbeitet werden, die zuvor durch die Zuweisung eines XBRLTaxonomieelements semantisch beschrieben wurden.

Damit sich XBRL und Services kombiniert nutzen lassen, ist im Rahmen eines als Mapping bezeichneten Vorgangs einmalig festzulegen, ${ }^{654}$ welche Objekte eines datenvorhaltenden IS mit welchen korrespondierenden Elementen einer in XBRL vorliegenden FR- oder GL-Taxonomie verknüpft werden können. ${ }^{655}$ Anschließend lassen sich aus dem IS die werttragenden Instanzdokumente unmittelbar erstellen.656 Nahezu alle führenden Datenbankhersteller haben eine derartige XML-Schnittstelle in ihre relationalen, objektrelationalen oder objektorientierten Produkte integriert. Nahe liegend erscheint darüber hinaus die Nutzung eines reinen XML-Datenbanksystems, das naturgemäß für die Speicherung von XML-Daten prädestiniert ist. ${ }^{657}$ In umgekehrter Weise bietet es sich

$652 \mathrm{Zu}$ den betriebswirtschaftlichen Einsatzmöglichkeiten von XBRL vgl. Gabriel/ Gluchowski/Pastwa (2006), S. 942f.

653 Vgl. Bovee et al. (2002), S. 166; Kemper/Mehanna/Unger (2004), S. 131.

654 Vgl. Dorloff/Leukel/Schmitz (2004a), S. 89f.; Dorloff/Leukel/Schmitz (2004b), S. 341 f.

655 Als Softwareunterstützung werden für diesen Zweck so genannte Mapping-Tools eingesetzt.

656 Vgl. Nutz/Strauß (2002), S. 454.

657 Zur XML-Unterstützung in Datenbanksystemen vgl. z. B. Schöning (2003), S. 166ff.; Klettke/Meyer (2003), S. $311 \mathrm{ff}$. 
an, die Geschäfts- und Finanzinformationen eingehender XBRL-Instanzen mit Hilfe eines Services auf die entsprechende Datenstruktur des IS zu verteilen.

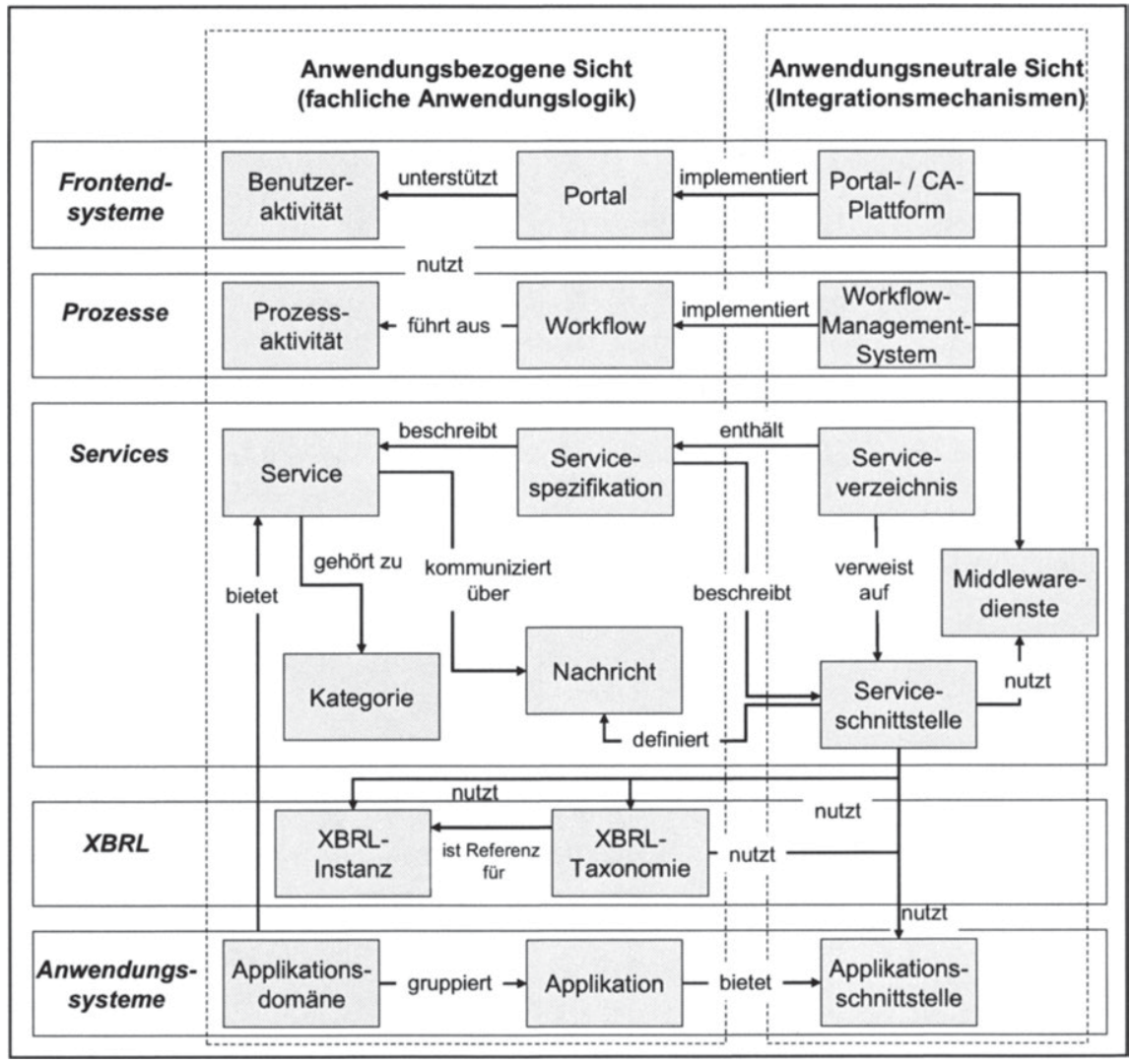

Abbildung 33: Erweiterung des SOA-Architekturmodells um die Ebene der Services

\subsection{Zusammenfassung und Würdigung des um XBRL erweiterten SOA- Architekturmodells}

Das vorliegende Kapitel fokussierte die Darstellung eines Architekturmodells, das sich zur Gestaltung und Ausführung von SOBP einsetzen lässt. In Abschnitt 4.1 wurde dazu der SOBP-Begriff präzisiert, der dieser Arbeit zugrunde liegt. Es wurde erklärt, dass sich ein SOBP dadurch auszeichnet, dass die Aktivitäten und Teilprozesse der Kernprozesse Informationsbeschaffung, Berichtserstel- 
lung, Berichtsdistribution und Berichtsaufnahme in Form von Services realisiert werden. Die Services eines SOBP lassen sich als Berichtsservices bzw. Berichtsdienste bezeichnen. SOBP werden mit dem Ziel durchgeführt, die Störungsursachen zu minimieren, die die Effizienz und Effektivität des betrieblichen Reportings gefährden. ${ }^{658}$

Ausgehend von dem in Abschnitt 3.5 präsentierten Verständnis einer SOA wurde in Abschnitt 4.2 ein Ebenenmodell vorgestellt, das mit Blick auf die aktuellen SOA-Beiträge mehrheitlich vorgeschlagen bzw. eingesetzt wird. Mit XBRL ließ sich das Architekturmodell in Abschnitt 4.3 um einen derzeit intensiv diskutierten XML-basierten Standard erweitern, der in dem Architekturmodell die Aufgabe hat, die im Rahmen des Berichtswesens zu verarbeitenden Geschäfts- und Finanzinformationen semantisch zu beschreiben.

Die Forschungsbemühungen zur Weiterentwicklung von XBRL waren von dem Ziel geprägt, einen Standard zu schaffen, mit denen sich die zu verarbeitenden Geschäfts- und Finanzinformationen gemäß den Sprach- und Syntaxvorgaben von XML anhand geeigneter und eindeutiger Elemente beschreiben lassen. Das Ergebnis dieser Anstrengungen mündete in einer Reihe von XBRL-Taxonomien, die sich für die Zwecke des internen und externen Berichtswesens nutzen lassen. Aufbauend auf den Begriffsverständnissen thematisch relevanter Autoren wurde XBRL dabei als frei verfügbarer, plattform- und systemunabhängiger (technologieunabhängiger) Open-Source-Kommunikationsstandard für die automatisierte und softwareunterstützte Erstellung, Verbreitung und Auswertung monetärer und nicht monetärer Geschäfts- und Finanzinformationen definiert.

Im Ergebnis ist zu konstatieren, dass das erarbeitete Architekturmodell das strukturelle Fundament liefert, um die mit dem Einsatz einer SOA erhofften Einsparungspotenziale, die aus der angestrebten hohen Wiederverwendbarkeit der Services resultieren, zu realisieren.659 In diesem Zusammenhang sieht das Architekturmodell vor, dass sich die hierin eingebundenen Dienste durch den Einsatz eines Serviceverzeichnisses auffinden und funktionsorientiert einsetzen lassen. Auf diese Weise können die Services von einer beliebigen Nutzerzahl mehrfach aufgerufen werden.

Die Potenziale zur schnellen Anpassbarkeit und höheren Wiederverwendbarkeit der Dienste werden dadurch gesteigert, dass durch die Veröffentlichung der Serviceschnittstellen nicht nur die Nutzung, sondern auch die Wartung der Dienste erleichtert wird. In diesem Zusammenhang wird mit der Einhaltung des Abstraktionsprinzips beabsichtigt, eine redundante Erstellung von Berichtsservices zu vermeiden. Hierzu sind die Metadaten bereits bestehender Dienste über die Servicebeschreibungen entsprechend auszuwerten.

659 Vgl. Laartz (2008), S. 72. 
Festzuhalten ist, dass sowohl die Portal-Technologie als auch der Einsatz einer Workflow-Engine eine wichtige Bedeutung bei der Ausführung von SOBP aufweisen. Mit Hilfe eines Portals lassen sich einzelne Berichtsservices aufrufen und die zu einem Berichtsprozess verknüpften Dienste ausführen. Zudem besteht die Möglichkeit, Berichtsservices zur Verfügung zu stellen, die von unternehmungsexternen Nutzern aufgerufen werden können. Derartige Services könne beispielsweise die Abfrage einzelner Informationen einer Bilanz zum Ziel haben. Gleichzeitig haben unternehmungsexterne Anbieter von Services die Möglichkeit, ihre Dienste am Portal der SOA-betreibenden Unternehmung anzumelden bzw. zu registrieren und damit zur Nutzung freizugeben.

Die WMS-Komponente ermöglicht, dass die auszuführenden Berichtsprozesse in der korrekten Reihenfolge unter Beachtung aller Vorgaben ablaufen und dokumentiert werden. Dadurch lassen sich die Berichtsprozesse transparent gestalten sowie ihre Kontrolle gewährleisten. Darüber hinaus birgt der Einsatz einer Workflow-Engine das Potenzial, eine applikations- und/oder unternehmungsübergreifende SOBP zu flexibilisieren und/oder zu automatisieren.

Zusätzlich unterstützt das Architekturmodell die kombinierte Verwendung von XBRL und Services. Dienste lassen sich in diesem Zusammenhang nutzen, um die Geschäfts- und Finanzinformationen, die in den XBRL-Instanzen semantisch beschrieben sind, aufzunehmen, zu transformieren und das Ergebnis der Transformation nachfolgenden Berichtsservices zur Verfügung zu stellen. Dadurch, dass Services genutzt werden, die die Daten bestehender Berichtsanwendungen verarbeiten, lassen sich zudem die verschiedenen IS, die für die Zwecke des Berichtswesens zum Einsatz kommen, auch weiterhin in einer SOA nutzen. Dies bedeutet für die Unternehmungen die Aussicht auf steigende Investitionssicherheit. 660 


\section{Vorgehensmodell zur konzeptionellen Gestaltung Serviceorien- tierter Berichtsprozesse}

Nachdem im vorigen Kapitel ein Architekturmodell für SOBP präsentiert wurde, richtet sich das Erkenntnisziel des vorliegenden Kapitels auf die Frage, wie sich SOBP unter Wahrung einer methodischen Vorgehensweise konzeptionell gestalten lassen. Festzuhalten ist, dass sich für die methodische Entwicklung einer SOA bisher keine einheitliche Vorgehensweise durchsetzen konnte. ${ }^{661}$ In Abschnitt 5.1 werden zunächst bestehende Vorgehensstrategien zur Einführung oder Entwicklung einer SOA hinsichtlich ihrer Eignung untersucht, für die konzeptionelle Gestaltung von SOBP eingesetzt zu werden. Darauf aufbauend wird in Abschnitt 5.2 ein Ansatz erarbeitet, der sich zur konzeptionellen Gestaltung von SOBP nutzen lässt. Das Kapitel schließt in Abschnitt 5.3 mit einer Zusammenfassung und der kritischen Würdigung des Vorgehensmodells.

\subsection{Evaluation bestehender Vorgehensstrategien zur Gestaltung Service- orientierter Anwendungen}

Die Ausführungen dieses Abschnitts stellen die grundsätzlichen Strategien zur Gestaltung Serviceorientierter Anwendungen auf Basis einer SOA vor. Prinzipiell kommen für diesen Zweck drei Vorgehensweisen in Frage. Dem Bottomup-Ansatz (Abschnitt 5.1.1) als erste Strategievariante steht der Top-downAnsatz (Abschnitt 5.1.2) gegenüber. ${ }^{662}$ Als dritte Variante lassen sich die beiden Ansätze zu einer integrierten Vorgehensweise kombinieren, die sich als Meet-in-the-middle-Ansatz bezeichnen lässt (Abschnitt 5.1.3).

\subsubsection{Top-down-Ansatz}

Der Top-down-Ansatz zeichnet sich allgemein dadurch aus, dass der spezifische Informationsbedarf Ausgangspunkt für die Entwicklung eines IS darstellt.663 Alle für die Entwicklung relevanten Datenquellen werden nach Bedarf ausgewählt und dem System hinzugefügt. Die grundsätzliche Vorgehensweise bei der Top-down-Entwicklung einer SOA ist in Abbildung 34 verdeutlicht.

Charakteristisch für die Top-down-Vorgehensweise ist, dass Services aus den geschäftlichen Anforderungen heraus identifiziert und beschrieben werden. 664

661 Vgl. Beverungen/Knackstedt/Müller (2008), S. 222.

662 Vgl. Niemann et al. (2002), S. 426.

663 Vgl. Leser/Naumann (2007), S. 105.

664 Vgl. Müller (2007b), S. 145. 
Ausgehend von der Geschäftsstrategie einer Unternehmung wird zunächst die IT-Strategie formuliert. Die IT-Strategie gibt vor, welche strategischen Mittel der Informationstechnologie zur Umsetzung der Geschäftsstrategie zum Einsatz kommen sollen. In diesem Zusammenhang ist über die technologische Plattform und damit über die zum Einsatz kommenden Software- und Hardwaresysteme zu entscheiden. Darüber hinaus ist die Wahl einer geeigneten Innovationsstrategie sowie einer Sourcing-Strategie, welche die Verteilung von internem und externem Know-how zum Gegenstand hat, von Bedeutung. Ferner muss eine ITStrategie die Regeln zur Freigabe von IT-Investitionen enthalten. Die Inhalte bzw. Vorgaben der IT-Strategie münden in der Definition einer IT-Architektur. 665

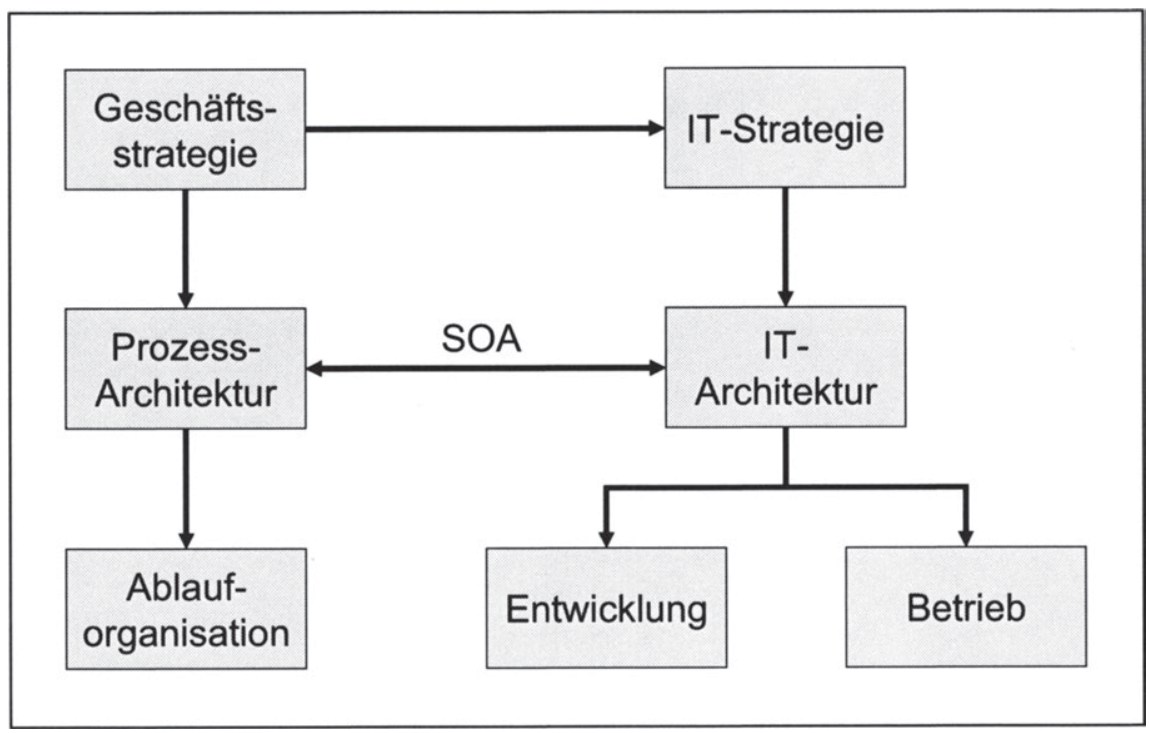

Abbildung 34: Top-down-Ansatz zur Einführung einer SOA

Neben der IT-Strategie und der IT-Architektur ist zur Operationalisierung der Geschäftsstrategie die Architektur der Geschäftsprozesse bzw. die ProzessArchitektur festzulegen. Eine Prozess-Architektur beschreibt den Gesamtzusammenhang der Leistungsentwicklung, Leistungserstellung und des Leis- 
tungsvertriebs in einer Organisation. ${ }^{666}$ Die Prozess-Architektur wirkt sich wiederum auf die Strukturierung der Ablauforganisation der SOA-betreibenden Unternehmung aus. ${ }^{667}$ Aus Abbildung 34 ist zu erkennen, dass SOA das integrierende Element zwischen der Prozess- und IT-Architektur darstellt. Als Architekturkonzept, das die Prozess- und die IT-Sicht verbindet, ermöglicht eine SOA durch einen Top-down-Ansatz, die Logik der Geschäftsprozesse unverändert in Form von orchestrierten Services zu implementieren. 668

Mit Blick auf die Vorgehensweise zur Gestaltung einer Serviceorientierten Anwendung ist festzuhalten, dass beim Top-down-Konzept die Analyse der Geschäftsprozesse sowie die schrittweise Ableitung von Services im Mittelpunkt stehen.669 Der Geschäftsprozessanalyse geht die Erstellung eines Anforderungskatalogs voraus, in welchem alle wesentlichen Kriterien an das zu entwerfende IS formuliert sind. Idealerweise liegt bereits ein Prozessmodell vor, das sich zur fachlichen Gestaltung und Implementierung der Services nutzen lässt. Liegt ein Prozessmodell nicht vor, muss es zunächst erstellt werden. ${ }^{670}$

Ausgehend von dem zu unterstützenden Geschäftsprozess, d. h. aus einer fachlichen Perspektive heraus, werden die entsprechenden Services identifiziert, entworfen, implementiert und in ein Verzeichnis eingebunden. Wie aus Abbildung 35 hervorgeht, ist es für diesen Zweck erforderlich, die zu unterstützenden Geschäftsprozesse schrittweise zu verfeinern und in Module bzw. Teilprozesse aufzuteilen. Die Modularisierung kann in Abhängigkeit von der Komplexität des Prozesses mehrere Detaillierungsebenen umfassen. ${ }^{671}$ Die Zerlegung der Geschäftsprozesse ist abgeschlossen, wenn die verfeinerten Prozess-

666 Vgl. Schelp/Hafner/Winter (2006), S. 214. Mit Hilfe einer Prozess-Architektur lassen sich die komplexen Zusammenhänge der Prozesse strukturiert erfassen. Zur Visualisierung, Analyse und Verbesserung werden die Prozesse in einem Modell mit vernetzten Ebenen und unterschiedlichen Detaillierungsstufen abgebildet, wobei jede Ebene der Prozess-Architektur unterschiedliche Aspekte und Zielgruppen adressiert. Vgl. Krcmar (2003), S. 261.

667 Unter Ablauforganisation wird die räumliche und zeitliche Ordnung von Geschäftsprozessen verstanden, die innerhalb einer Aufbauorganisation vorliegt. Die Aufbauorganisation einer Unternehmung legt die Zuständigkeiten für die arbeitsteilige Aufgabenerfüllung fest und beschreibt die Organisationseinheiten sowie die zwischen den Organisationseinheiten existierenden Beziehungen zueinander. Vgl. Oestereich/Weiss (2008), S. $416 f$.

668 Vgl. Liebhart (2007), S. 173.

669 Vgl. hierzu von Henning (2007), S. 343.

670 OEHLER bemängelt in diesem Zusammenhang, dass generell in vielen Unternehmungen keine umfassenden Prozessdokumentationen vorliegen oder die Prozesse nur unzureichend dokumentiert sind. Vgl. Oehler (2006), S. 104.

671 Vgl. Gimnich (2007), S. 72. 
module eine Granularität erreichen, die der Granularität der Services auf der Geschäftsebene entspricht.672

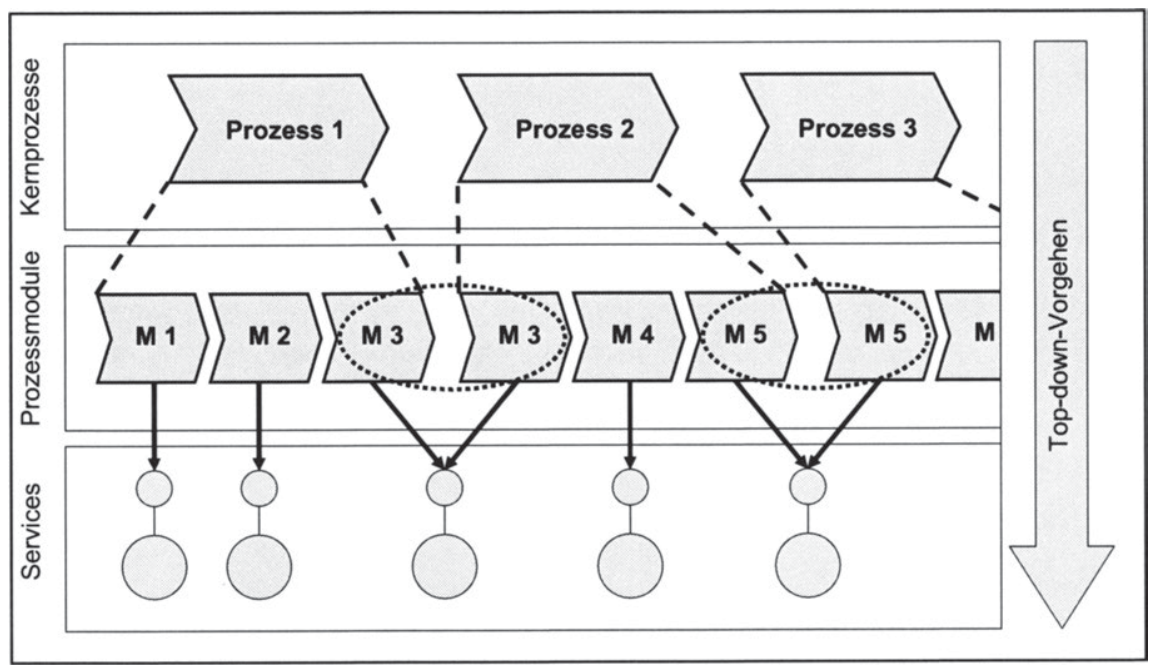

Abbildung 35: Vorgehen beim Top-down-Ansatz ${ }^{673}$

Eine wichtige Eigenschaft der Top-down-Vorgehensweise ist, dass gleichartige Prozessmodule identifiziert, zusammengefasst und schließlich durch Services implementiert werden. ${ }^{674}$ Diese Konstellation ist in dem in Abbildung 35 betrachteten Beispiel bei den Prozessmodulen M3 und M5 gegeben. Ziel dieses Vorgehens ist es, Redundanzen zu vermeiden und eine hohe Wiederverwendung von Diensten zu erzielen. Die Dienste erfüllen eine abgegrenzte fachliche Aufgabe und orientieren sich nicht an den IT-Applikationen, die von der SOAbetreibenden Unternehmung genutzt werden, ${ }^{675}$ sondern an den Aktivitäten der auszuführenden Geschäftsprozesse.

\subsubsection{Bottom-up-Ansatz}

Beim Bottom-up-Ansatz erfolgt eine Umkehrung der Teilschritte, die bei der Vorgehensweise der Top-down-Strategie durchlaufen werden. In Abgrenzung zur Top-down-Strategie steht beim Bottom-up-Ansatz generell die Integration

672 Vgl. Höß et al. (2007), S. 42. Zum Begriff der Granularität vgl. Abschnitt 5.2.2.1.

673 In Anlehnung an Höß et al. (2007), S. 43.

674 Vgl. Höß et al. (2007), S. 43.

675 Vgl. Müller (2007b), S. 145. 
bestehender und bekannter Datenquellen und Funktionalitäten im Vordergrund, die von den IS bereits zur Verfügung gestellt werden. ${ }^{676}$ Charakteristisch für diesen Ansatz ist, dass bei der Gestaltung einer Anwendung mit den Systemteilen auf der untersten Ebene eines hierarchisch gegliederten Systems begonnen wird. ${ }^{677}$ Beim Bottom-up-Ansatz handelt es sich folglich um ein eher technisch orientiertes Vorgehen. 678

Mit Blick auf die Gestaltung einer Serviceorientierten Anwendung stellen beim Bottom-up-Ansatz nicht die zu unterstützenden Geschäftsprozesse den Ausgangspunkt dar. Stattdessen fokussiert diese Vorgehensweise das bestehende Service- sowie Anwendungssystemportfolio einer Unternehmung.679 GIMNICH stellt in diesem Zusammenhang fest, dass sich viele Services aus den bestehenden Awendungen gewinnen lassen. ${ }^{680}$ Der Bottom-up-Ansatz wird durch Abbildung 36 verdeutlicht.

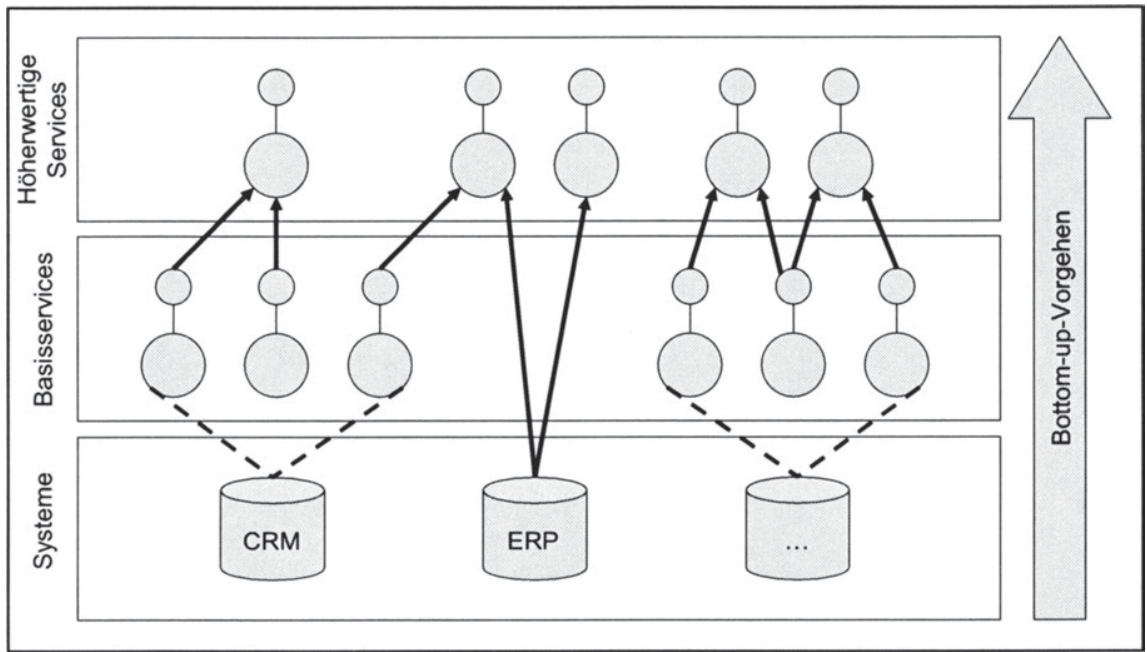

Abbildung 36: Vorgehen beim Bottom-up-Ansatz ${ }^{681}$

676 Vgl. Leser/Naumann (2007), S. 105; Höß et al. (2007), S. 44.

677 Vgl. Heinrich (2002), S. 94.

678 Vgl. Dorda/Steiert/Sellentin (2004), S. 203.

679 Vgl. Liebhart (2007), S. 173; Müller (2007b), S. 146.

680 Vgl. Gimnich (2007), S. 68. Zu diesen Services gehören z. B. die Angebotserstellung, die Neukundenanlage und die Ermittlung von Konditionen.

681 In Anlehnung an Höß et al. (2007), S. 44. Im Gegensatz zu der von HÖß ET AL. präsentierten Abbildung wurden in Abbildung 36 die Pfeile von den IS auf die Services gerichtet. Die dadurch angedeutete Vorgehensweise, aus den IS Services zu gestalten, entspricht dem Vorgehen nach dem Bottom-up-Ansatz. 
Wie aus der Abbildung ersichtlich, sind zunächst die von einer Unternehmung betriebenen Anwendungssysteme vor dem Hintergrund der Frage zu untersuchen, welche bereits bestehenden Funktionalitäten als potenzielle Services implementiert sind. ${ }^{682}$ Aus diesen Services lassen sich gegebenenfalls neue Dienste ableiten, die mit weiteren Services verknüpft werden können, um die geforderte Leistung zu erbringen. ${ }^{683}$ Liegen keine implementierten Services vor, empfiehlt es sich, zunächst wichtige Funktionalitäten, die von den bestehenden IS erbracht werden, als individuelle Services zu realisieren. ${ }^{684} \mathrm{Zu}$ diesem Zweck werden die bestehenden IS in Anwendungsdomänen zerlegt, ${ }^{685}$ aus denen sich entsprechende Services erstellen lassen. Aus diesen Services lassen sich gegebenenfalls neue Dienste ableiten, die mit weiteren Services verknüpft werden können, um die anvisierten Dienste bereitzustellen.686 Die individuellen Services übernehmen in diesem Fall die Aufgabe eines Basisservices für höherwertige Dienste, die eine wichtige Geschäftsfunktion erbringen.687 In Abbildung 36 ist $\mathrm{zu}$ erkennen, dass sich derartige Dienste aus verschiedenen Basisservices zusammensetzen.

Eine Erweiterung des Serviceportfolios erfolgt dann schrittweise. D. h., durch die folgenden Schritte sind Services bereitzustellen, welche Funktionen erbringen, die von weiteren Applikationen genutzt werden können. In einem vorletzten Schritt wird geprüft, inwieweit sich einzelne Services gruppieren oder zu einem übergeordneten Dienst verknüpfen lassen. Diese Services bieten das Potenzial, als Aktivitäten eines Serviceorientierten Geschäftsprozesses eingesetzt zu werden. In einem letzten Schritt erfolgt bei der Bottom-up-Strategie die Konfiguration der Services zu Serviceorientierten Geschäftsprozessen.

682 Im Beispiel der Abbildung 36 werden z. B. IS untersucht, die für die Zwecke des CRM sowie des ERP genutzt werden.

683 Vgl. Müller (2007b), S. 146.

684 HÖß ET AL. bemerken in diesem Zusammenhang, dass moderne Systeme beispielsweise im ERP-Umfeld über die technische Möglichkeit verfügen, komplette Funktionalitäten in einer fachlichen und technischen Form zur Verfügung zu stellen, die einem Service im Sinne einer SOA entspricht. Vgl. Höß et al. (2007), S. 44.

685 Zum Domänenbegriff vgl. Abschnitt 5.2.1.1. Neben der organisatorisch geprägten Auslegung lässt sich der Domänenbegriff auch aus einer informationstechnischen Perspektive betrachten. Eine Domäne wird hier verstanden als Gruppierung logisch bzw. fachlich zusammengehörender Anwendungen, die jeweils über sich gegenseitig verbindende Schnittstellen verfügen müssen. Vgl. Gronau et al. (2008), S. 430 und 434.

686 Vgl. Müller (2007b), S. 146.

687 Als Geschäftsfunktion wird eine Aktion aufgefasst, die eine geschäftsrelevante Änderung zur Folge hat. Vgl. Tilkov/Starke (2007), S. 14. 


\subsubsection{Meet-in-the-middle-Ansatz}

Als drittes Vorgehensmodell zur Entwicklung einer SOA wird nun ein Ansatz vorgestellt, der sowohl Eigenschaften des Top-down-Ansatzes als auch Merkmale des Bottom-up-Konzeptes aufweist. Bei dieser als Meet-in-the-middle bezeichneten Variante wird eine parallele Vorgehensweise zur Implementierung der Dienste angewendet. ${ }^{688}$ Sowohl die Geschäftsprozesse als auch die Anwendungssysteme, die zur Ausführung der Geschäftsprozesse zum Einsatz kommen, werden analysiert. Folglich sind geeignete Services sowohl auf Basis der vorhandenen Anwendungssysteme nach Vorbild des Bottom-up-Ansatzes als auch auf Grundlage der Geschäftsprozesse im Sinne des Top-down-Prinzips zu erstellen bzw. gegebenenfalls zu modifizieren.

Abbildung 37 stellt das Vorgehen des Meet-in-the-middle-Ansatzes dar. ${ }^{689}$ In einem ersten Schritt müssen die von der Unternehmung betriebenen IS auf ihre Zukunftsfähigkeit analysiert und potenzielle Services identifiziert werden. Der zweite Schritt sieht vor, aus den Hauptprozessen Prozessmodule zu bilden, die zur Ableitung von höherwertigen Services dienen. In einem dritten Schritt ist zu untersuchen, welche höherwertigen Dienste sich durch Basisservices der bereits bestehenden IS abbilden lassen. Ist diese Möglichkeit nicht oder nur eingeschränkt gegeben, muss entschieden werden, welche Services neu zu entwickeln sind.

Wie in der Abbildung 37 deutlich wird, ist beim Meet-in-the-middle-Ansatz die Ebene der höherwertigen Services von Bedeutung, da sich hier sowohl die fachliche als auch die IT-Sicht treffen. Auf diese Weise lassen sich die Geschäftsprozesse einerseits nach fachlichen Aspekten verbessern, andererseits können die IS und Services unterhalb der Ebene der höherwertigen Services nach IT-orientierten Gesichtspunkten verbessert werden. ${ }^{690}$ Potenziale ergeben sich z. B. dadurch, dass eine gemeinsame Datenhaltung für Kundendaten verschiedener Geschäftsbereiche durch die IT-orientierte Verbesserung ermöglicht wird.

688 Vgl. Liebhart (2007), S. 174.

689 Siehe hierzu die Ausführungen von Höß et al. (2007), S. 44f.

690 Vgl. Höß et al. (2007), S. 45. 


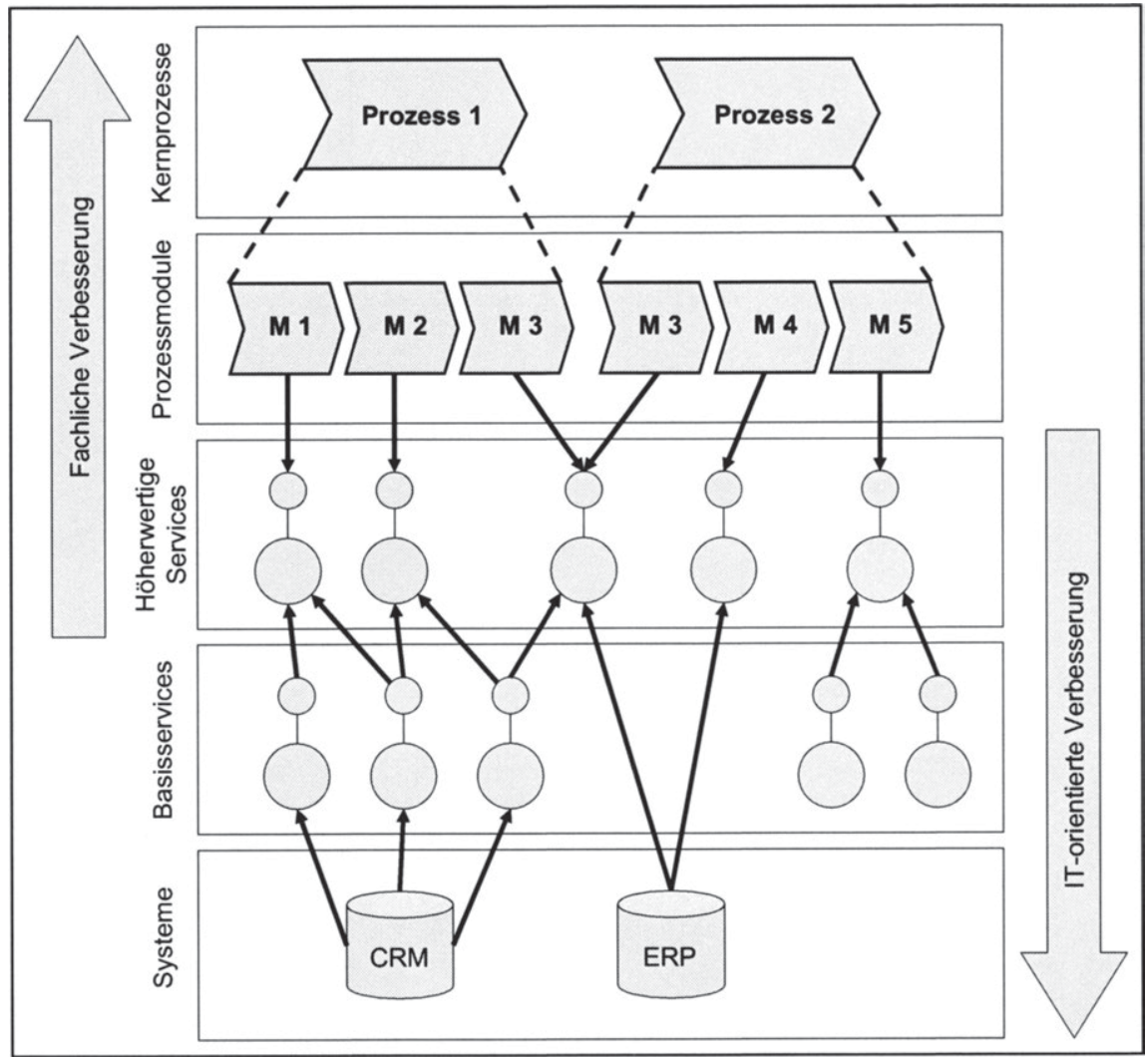

Abbildung 37: Meet-in-the-middle-Ansatz 691

\subsubsection{Bewertung der bestehenden Ansätze für die konzeptionelle Gestal- tung Serviceorientierter Berichtsprozesse}

Hinsichtlich der praktischen Anwendbarkeit des Top-down- oder Bottom-upAnsatzes zur konzeptionellen Gestaltung eines SOBP ist zu konstatieren, dass beide Ansätze in Abhängigkeit der vorliegenden Rahmenbedingungen als eigenständige Konzepte sinnvoll sind.

Für die Bottom-up-Vorgehensweise spricht, dass auf diese Weise keine Services übersehen werden können, ${ }^{692}$ die bereits in der Unternehmung realisiert wurden und im Hinblick auf das Anwendungsszenario zum Einsatz kommen

691 In Anlehnung an Höß et al. (2007), S. 44.

692 Vgl. Gimnich (2007), S. 73. 
können. Eine Begründung für eine Vorgehensweise nach dem Bottom-upAnsatz lässt sich zudem in den Anwendungsfällen identifizieren, bei denen bereits eine Reihe von Anwendungssystemen, in denen große Mengen wertvoller und unternehmungskritischer Daten vorliegen bzw. verarbeitet werden, von einer Unternehmung betrieben werden.693 Diese Applikationen lassen sich als Komponenten einer Serviceorientierten Anwendung verwenden oder erweitern. ${ }^{694}$ Mit Blick auf den Anwendungsbereich dieser Arbeit liegt eine derartige Situation in einer Unternehmung in der überwiegenden Mehrheit vor, da die Geschäfts- und Finanzinformationen, die bei der Ausführung der Berichtsprozesse verarbeitet werden, nicht nur in vielen operativen und analyseorientierten IS vorgehalten, sondern auch mit Hilfe diverser Analysetools ausgewertet werden.

Da der Bottom-up-Ansatz die „behutsame“ Einführung einer SOA anstrebt, sind im Hinblick auf die Amortisation der Anfangsinvestitionen schnellere und unmittelbare, kleine Erfolge zu erwarten. ${ }^{695}$ Darüber hinaus trägt das Bottomup-Prinzip zu einer effektiven Lernphase bei den Mitarbeitern und einem parallelen Aufbau einer technischen Umgebung, von personellem Know-how und organisatorischen Strukturen bei. 696

Im Vergleich zur Top-down-Strategie zeichnet sich der Bottom-up-Ansatz zudem durch eine pragmatische Herangehensweise aus. Dies führt allerdings zur Gefahr, dass im Ergebnis keine geschlossene Gesamtarchitektur im Sinne einer SOA erstellt wird, ${ }^{697}$ bei der alle Geschäftsprozesse mit Hilfe verknüpfter Services ausgeführt werden. Dies ist als Nachteil zu werten, da beim Betrieb einer SOA SOBP nur eine von vielen Prozesskategorien darstellen. Infolge der fehlenden Prozessorientierung beim Bottom-up-Vorgehen ist ein „Sammelsurium" an Berichtsservices zu erwarten, welche je nach Anwendungsbereich bzw. Berichtsprozess punktuell entwickelt bzw. eingesetzt werden und nicht die angestrebte Wiederverwendbarkeit erreichen. Als weiterer Nachteil ist bei der Bottom-up-Strategie zu werten, dass der Fokus auf die Daten und die Funktionalität gelenkt wird, die von den bereits betriebenen Anwendungssystemen vorgehalten bzw. erbracht werden. Eine solch einengende Sicht birgt die Gefahr, dass die Nutzen stiftenden Potenziale von domänenübergreifenden Berichtsservices nicht vollständig erkannt und ausgeschöpft werden.

693 Vgl. Höß et al. (2007), S. 43. SieBENHAAR ET AL. bemerken in diesem Zusammenhang, dass ein Bottom-up-Vorgehen als sinnvoll zu werten ist, da Serviceorientierte Anwendungen i. d. R. nicht auf der "grünen Wiese“ entwickelt werden. Vgl. Siebenhaar et al. (2008), S. 325.

Vgl. Siebenhaar et al. (2008), S. 325.

695 Vgl. Ernst et al. (2008).

696 Vgl. Bark/Fritsch (2006).

697 Vgl. Bark/Fritsch (2006). 
Im Vergleich zum Bottom-up-Ansatz ist bei der Anwendung der Top-downStrategie der Nachteil zu identifizieren, dass es sich hierbei um eine investitionsintensivere Vorgehensweise handelt. ${ }^{698}$ Hohe Aufwendungen entstehen, da sämtliche zu unterstützenden Berichtsprozesse modelliert bzw. angepasst werden müssen. Neben den hohen Anfangskosten ergeben sich zudem sowohl Gefahren unternehmungskultureller Verwerfungen und Widerstände als auch Tücken einer zu hastigen und damit unzureichenden Organisation.699 In diesem Zusammenhang ist zu bemerken, dass die konsequente Ausrichtung auf die Berichtsprozesse und die damit verbundene ,plötzliche" Umstellung der gesamten Arbeitsumgebung in Form einer „Big-Bang“-Lösung zu einer Überforderung der Mitarbeiter führen kann. Zu erwarten sind hierdurch weitere Effizienzeinbußen. $\mathrm{Zu}$ diesem Problemfeld trägt auch das Nichtvorhandensein einer Lernphase bei. Ferner sind durch die erhöhte Komplexität der Abhängigkeiten zwischen den Berichtsservices verlängerte Gesamtlaufzeiten eines entsprechenden Projekts zu erwarten, die sich wie bei anderen SOA-Projekten wiederum auf einen niedrigen ROI auswirken. ${ }^{700}$

Die von BEVERUNGEN/KNACKSTEDT/MÜLLER durchgeführte Gegenüberstellung verschiedener Ansätze zur Gestaltung einer SOA führt zum Ergebnis, dass in der Literatur der Top-down- und der Meet-in-the-middle-Ansatz favorisiert werden. ${ }^{701}$ Das Vorgehensmodell, das zur Gestaltung eines SOBP zum Einsatz kommen soll, muss in jedem Fall berücksichtigen, dass möglicherweise bereits Services in der Unternehmung existieren, die sich für die Zwecke des Reportings nutzen lassen und daher nicht neu implementiert werden müssen. Ebenso muss das Vorgehen berücksichtigen, dass in einer Unternehmung typischerweise bereits verschiedene Berichtsanwendungen genutzt werden, die nicht nur berichtsrelevante Geschäfts- und Finanzinformationen vorhalten, sondern auch wiederverwendbare Funktionen in Form von Services zur Verfügung stellen.

Für ein Top-down-gestütztes Vorgehen spricht, dass mit den in Abschnitt 2.2.2 identifizierten Kernprozessen vier Prozesskategorien vorliegen, die für die Modellierung eines vollständigen Berichtsprozesses als Ausgangspunkt dienen können. Damit eröffnet sich der Unternehmung die Möglichkeit, dem Leitgedanken einer SOA folgend, die Prozessmodellierung losgelöst von den zugrunde liegenden Anwendungssystemen zu betrachten. Ferner ergibt sich bei diesem Vorgehen der Vorteil, dass eine kohärente Umsetzung einer Berichtsstrategie ermöglicht wird. ${ }^{702}$ Wird die konzeptionelle Gestaltung eines SOBP auf Basis

Vgl. Liebhart (2007), S. 173.

Vgl. Bark/Fritsch (2006).

Vgl. Ernst et al. (2008).

gl. Beverungen/Knackstedt/Müller (2008)

702 LIEBHART spricht in diesem Zusammenhang allgemein von einer Geschäftsstrategie. Vgl. Liebhart (2007), S. 173. 
einer SOA angestrebt, ist mit Blick auf diese Aspekte die Top-down-Strategie dem Bottom-up-Ansatz vorzuziehen.

Um diesen Erwägungen Rechnung zu tragen, wird in dieser Arbeit die Auffassung vertreten, dass das im Folgenden entwickelte Vorgehensmodell zur Gestaltung eines SOBP sowohl den in der Wissenschaft favorisierten Topdown-Ansatz als auch den pragmatischen Bottom-up-Ansatz integrieren muss. Damit kommt als geeigneter Ansatz der Meet-in-the-middle-Ansatz in Frage. ${ }^{703}$

\subsection{Konzeptionelle Gestaltung Serviceorientierter Berichtsprozesse}

Aufbauend auf den vorherigen Ausführungen verfolgt der vorliegende Abschnitt das Ziel, einen Ansatz zu präsentieren, der sich zur konzeptionellen Gestaltung eines SOBP einsetzen lässt. Dabei repräsentiert die gewählte Gliederung bereits die dreistufige Vorgehensweise: Zunächst wird in Abschnitt 5.2.1 die Berichtsprozessanalyse aus der Perspektive der Serviceorientierung thematisiert. Die berichtsprozessmodellbasierte Serviceidentifikation ist dann Gegenstand des Abschnitts 5.2.2, während die fachliche Gestaltung von Berichtsservices in Abschnitt 5.2.3 diskutiert wird.

Mit Blick auf die in Abbildung 34 dargestellte Top-down-Vorgehensweise ist in diesem Zusammenhang festzuhalten, dass die folgenden Ausführungen zur konzeptionellen Gestaltung eines SOBP nicht die Phasen „Ableitung einer Geschäftsstrategie“ und „Ableitung einer IT-Strategie" thematisieren, sondern direkt bei der Berichtsprozessanalyse ansetzen. Die Phasen „Ableitung einer Geschäftsstrategie“ und „Ableitung einer IT-Strategie“ werden nicht behandelt, da sie Schritte darstellen, die unabhängig von den zu unterstützenden Geschäftsprozessen bei der Entwicklung einer SOA durchzuführen sind. Vor dem Hintergrund der im Rahmen dieser Arbeit untersuchten Fragestellung fokussieren die folgenden Ausführungen stattdessen die Phasen „Berichtsprozessanalyse“, „Berichtsserviceidentifikation“ und „Berichtsservicespezifikation“ als Kernphasen der konzeptionellen Gestaltung eines SOBP.

\subsubsection{Berichtsprozessanalyse aus der Perspektive der Serviceorientierung}

Unter dem Begriff „Berichtsprozessanalyse“ lassen sich alle vorbereitenden und unterstützenden Aktivitäten zur Zielbestimmung, Modellierung sowie Anpassung eines Berichtsprozesses verstehen, die im Vorfeld der Identifikation von Berichtsservices durchgeführt werden müssen. In Abschnitt 5.2.1.1 werden Kategorien vorgestellt, die sich zur Zielbestimmung des zu gestaltenden SOBP

703 Damit wird der Auffassung von Höß ET AL. entsprochen, zwecks einer Erfolg versprechenden und praxisorientierten Vorgehensweise für die Identifikation und Definition von Diensten beide Ansätze in Kombination anzuwenden. Vgl. Höß et al. (2007), S. 45.

Alexander Pastwa -978-3-631-75488-7 
nutzen lassen. Aspekte der Berichtsprozessmodellierung sowie -anpassung werden in Abschnitt 5.2.1.2 erörtert. Die Einbindung und Bewertung unternehmungsexterner Berichtsservices in ein Serviceverzeichnis sowie die Evaluation bestehender unternehmungsinterner Berichtsservices ist Gegenstand der Ausführungen von Abschnitt 5.2.1.3.

\subsubsection{Zielbestimmung der zu gestaltenden Serviceorientierten Berichts- prozesse anhand eines Ordnungsrahmens}

Die Zielbestimmung eines SOBP lässt sich anhand der Dimensionen „Berichtsempfänger“, „Verantwortlichkeit“, „Strukturierungsgrad des Workflows“, „Auslöser des Reportings“ sowie „Berichtszweck“ durchführen. Einen Überblick über die Ausprägungen dieser Dimensionen präsentiert Abbildung 38.

\begin{tabular}{|c|c|c|c|c|}
\hline Dimension & \multicolumn{4}{|c|}{ Ausprägung } \\
\hline Verantwortlichkeit & Geschäftszweig & Geschäftseinheit & Abteilung & Team \\
\hline $\begin{array}{c}\text { Strukturierungs- } \\
\text { grad }\end{array}$ & $\begin{array}{c}\text { Ad-hoc- } \\
\text { Workflow }\end{array}$ & $\begin{array}{r}\text { Semi-strukturierter } \\
\text { Workflow }\end{array}$ & $\begin{array}{c}\text { Vollständig definierter } \\
\text { Workflow }\end{array}$ \\
\hline $\begin{array}{c}\text { Auslöser des } \\
\text { Reporting }\end{array}$ & \multicolumn{3}{|c|}{ Aktives Reporting } & \multicolumn{2}{|c|}{ Passives Reporting } \\
\hline $\begin{array}{c}\text { Berichtszweck } \\
\text { Berichts- } \\
\text { emptänger }\end{array}$ & $\begin{array}{c}\text { Dokumentation von } \\
\text { Ereignissen }\end{array}$ & $\begin{array}{c}\text { Auslösung von } \\
\text { Aktivitäten } \\
\text { Operatives }\end{array}$ & $\begin{array}{c}\text { Vorbereitung und } \\
\text { Kontrolle von } \\
\text { Entscheidungen }\end{array}$ \\
\hline
\end{tabular}

Abbildung 38: Dimensionen zur Zielbestimmung eines SOBP

\section{Berichtsempfänger}

Vor dem Hintergrund der in dieser Arbeit zugrunde gelegten weiten Auslegung des Berichtswesens ist zu konstatieren, dass ein SOBP sowohl auf den Informationsbedarf unternehmungsinterner als auch -externer Berichtsempfänger ausgerichtet sein kann. Als potenzielle unternehmungsexterne Berichtsempfänger kommen sowohl die Stakeholder als auch die Shareholder in Frage. ${ }^{704}$

Eine Einteilung der unternehmungsinternen Berichtsempfänger(gruppen) lässt sich anhand der Managementebenen durchführen. $\mathrm{Zu}$ unterscheiden ist das 
operative, taktische und strategische Management. ${ }^{705}$ Manager, die der operativen Ebene angehören (Lower-Management), sind für ihre Aufgabenerfüllung auf historische und/oder aktuelle sowie quantitative Informationen angewiesen. ${ }^{706}$ Derartige Informationen lassen sich i. d. R. aus den unternehmungsinternen IS gewinnen. Das strategische Management (Top-Management) fokussiert indessen qualitative und aggregierte Informationen. Dabei werden auch unternehmungsexterne Informationen nachgefragt. Während sich der tendenziell stabile Informationsbedarf des operativen Managements mit Hilfe eines klassischen Berichtswesens befriedigen lässt, variiert der Informationsbedarf des strategischen Managements stark. Dies ist der Tatsache zu schulden, dass die bereitgestellten Informationen eher sporadisch bzw. unregelmäßig genutzt werden. Der Informationsbedarf des taktischen Managements (Middle-Management) liegt zwischen den Informationsanforderungen des operativen und strategischen Managements.

\section{Verantwortlichkeit}

$\mathrm{Da}$ an einem Berichtsprozess nicht nur verschiedene Personen mit unterschiedlichen Verantwortungsbereichen und Aufgaben partizipieren können, sondern auch diverse IS genutzt werden, liegt ein weiterer Gestaltungsparameter eines SOBP in seiner organisatorischen Reichweite. Mit Blick auf die organisatorische Reichweite eines SOBP sind die Verantwortlichkeiten für die Berichtsservices und IS, die bei der Ausführung der SOBP genutzt werden, festzulegen. Zur Gruppierung der zum Einsatz kommenden IS und Akteure wird der Domänenbegriff eingeführt. Eine Domäne steht für eine „natürliche“ Unternehmungseinheit innerhalb der Systemlandschaft mit klaren Verantwortlichkeiten, die in der Organisationsstruktur eingebettet ist. ${ }^{707}$ Als potenzielle Domänen, die in einem SOBP für die Berichtserstellung, -verteilung oder den -empfang verantwortlich sein können, kommen z. B. die Leiter eines bestimmten Geschäftszweigs, einer Geschäftseinheit, einer Abteilung oder eines Teams innerhalb einer Unternehmung in Frage.

Zur Analyse der organisatorischen Reichweite eines SOBP lässt sich ein unternehmungsinterner von einem unternehmungsübergreifenden SOBP abgrenzen. Ein unternehmungsinterner SOBP umfasst alle Informationsflüsse sowie Vorgänge zur Verarbeitung der Geschäfts- und Finanzinformationen, die von den Domänen innerhalb einer Unternehmung geplant, durchgesetzt und gesteuert werden. Die Ausrichtung auf die unternehmungsinternen Prozesse, Personen

705 Vgl. hierzu Gluchowski/Gabriel/Chamoni (1997), S. 73f.

$706 \mathrm{Zu}$ den Aufgaben des operativen, taktischen und strategischen Managements vgl. Hellriegel/Jackson/Slocum (1999), S. 11 ff.; Gluchowski/Gabriel/Chamoni (1997), S. 9 und $168 \mathrm{ff}$.

707 Vgl. Josuttis (2008), S. 135. 
und IS ist aber nicht gleichbedeutend mit der ausschließlichen Nutzung derartiger Prozesse für das interne Berichtswesen. In gleichem Maße lässt sich eine unternehmungsinterne SOBP auch auf die Produktion von Berichten ausrichten, welche den Informationsbedarf unternehmungsexterner Anspruchsgruppen fokussieren.

Mit Blick auf den in diesem Abschnitt präsentierten Domänenbegriff wird eine unternehmungsinterne SOBP auf einer tieferen Betrachtungsebene in die $\mathrm{Ka}-$ tegorien geschäftszweiginterne und geschäftszweigübergreifende SOBP eingeteilt. Da ein Geschäftszweig aus mehreren Geschäftseinheiten bestehen kann, lässt sich auf einer weiteren Detaillierungsstufe ein geschäftseinheitsinterner von einem geschäftseinheitsübergreifenden Berichtsprozess unterscheiden. Zur weiteren Systematisierung eines geschäftseinheitsinternen SOBP kommt die Abgrenzung eines abteilungsinternen gegenüber einem abteilungsübergreifenden SOBP in Frage. Auf der tiefsten Betrachtungsebene ist es denkbar, bei einem abteilungsinternen SOBP einen teaminternen von einem teamübergreifenden dienstorientierten Berichtsprozess zu separieren.

Als besondere Ausprägung eines unternehmungsinternen SOBP lässt sich ein konzerninterner dienstorientierter Berichtsprozess anführen. Hierbei handelt es sich um einen Berichtsprozess, in dem - je nach Ausprägung der Konzernstruktur - Domänen partizipieren können, die sowohl zur Konzernmutter als auch zu den Konzerntöchtern gehören.

Im Vergleich zu einem unternehmungsinternen SOBP richtet sich der Gegenstandsbereich eines unternehmungsübergreifenden SOBP auf die Einbindung von Berichtsservices, die von unternehmungsexternen Domänen bereitgestellt werden. Neben einzelnen Services können auch Teilprozesse, die auf orchestrierten Services basieren, von einer unternehmungsexternen Domäne erbracht und mit den Teilprozessen der berichtenden Unternehmung vernetzt werden. Wie bei einer unternehmungsinternen SOBP lassen sich ebenfalls die unternehmungsübergreifenden SOBP sowohl für die Zwecke des internen als auch externen Reporting gestalten.

\section{Strukturierungsgrad}

Da SOBP aus mehreren Berichtsservices bestehen, die sich - dem SOAGedanken folgend - mit Hilfe eines WMS zu einer Serviceorientierten Berichtskette zusammenfügen lassen, ist es möglich, vollständige Berichtsprozesse sowie Teilprozesse in unterschiedlicher Art und Weise zu automatisieren. Mit Blick auf den Automatisierungsgrad von Workflows lassen sich hierbei die Ausprägungen Ad-hoc-Workflow, Semi-strukturierter Workflow sowie vollständig definierter Workflow unterscheiden. ${ }^{708}$ 
Vollständig definierte Workflows zeichnen sich durch den höchsten Automatisierungsgrad aus, da sie eine hohe Wiederholungsfrequenz aufweisen. Alternativ wird für diese Workflowkategorie der Terminus Production-Workflow verwendet. Semi-strukturierte Workflows, die auch als administrative Workflows bezeichnet werden, haben eine niedrigere Wiederholungsfrequenz. Im Vergleich zu den vollständig definierten Workflows sind semi-strukturierte Workflows weniger komplex und haben eine geringe Zahl von Workflowbeteiligten. Charakteristisch für Ad-hoc-Workflows ist, dass sie lediglich einmal ausgeführt werden. Von allen Workflowtypen haben sie die geringste Wiederholungsfrequenz. I. d. R. entstehen Ad-hoc-Workflows im Rahmen der täglichen Arbeit. Sie sind folglich typischerweise nicht vollständig planbar. In Abgrenzung zu den erst genannten Prozessen werden Ad-hoc-Workflows daher auch als schwach strukturierte Prozesse bezeichnet.

\section{Auslöser des Reportings}

Ferner muss festgelegt werden, ob der SOBP nach dem Vorbild einer aktiven oder passiven Informationsversorgung ausgeführt werden soll. In diesem $\mathrm{Zu}$ sammenhang ist zu bestimmen, ob der jeweils betrachtete SOBP für ein Standard- oder Ad-hoc-Reporting zur Anwendung kommen soll. Während sich zur Erfüllung des Berichtszwecks „Dokumentation von Ereignissen“ ein StandardReporting eignet, lässt sich der Berichtszweck ,Auslösung von Aktivitäten“ mit Hilfe eines Ad-hoc-Reportings realisieren. Zur Vorbereitung und Kontrolle von Entscheidungen lassen sich beide Reporting-Konzepte nutzen.

\section{Berichtszweck}

Das letzte Kriterium zur Unterscheidung eines SOBP orientiert sich an den Berichtszwecken, die mit den zu gestaltenden Berichtsprozessen verfolgt werden. Mit Blick auf die Ausführungen in Abschnitt 2.1.3 lassen sich hierzu sowohl unternehmungsinterne als auch -externe SOBP zur Dokumentation von Ereignissen, Auslösung von Aktivitäten sowie Vorbereitung und Kontrolle von Entscheidungen einsetzen.

\subsubsection{Modellierungsmethodengestützte Erstellung neuer Berichtspro- zesse oder Veränderung bestehender Berichtsprozesse}

Bevor die Identifikation sowie die fachliche Gestaltung der Berichtsservices beginnt, muss sichergestellt werden, dass ein Berichtsprozess auch tatsächlich 
vorliegt. ${ }^{709}$ Liegt kein Berichtsprozess vor, so muss ein solcher erstellt werden. Existiert bereits ein Berichtsprozess, ist zu prüfen, inwieweit sich dieser für die weitere Betrachtung eignet oder zu überarbeiten ist.

\subsection{Vorgehen}

Damit aus einem Berichtsprozess geeignete Berichtsservices, die sich zu einem SOBP verknüpfen lassen, abgeleitet werden können, muss der zu gestaltende Berichtsprozess systematisch solange in Teilprozesse zerlegt werden, bis eine weitere Aufspaltung der Teilprozesse aus funktioneller Sicht nicht mehr sinnvoll ist. Die elementaren Teilprozesse bzw. die in ihnen enthaltenen Basisaktivitäten dienen im Anschluss als Ausgangspunkt für die Identifizierung der $\mathrm{Be}$ richtsservices. Als Startpunkt zur Zerlegung des Berichtsprozesses lassen sich die Prozesse Informationsbeschaffung, Berichtserstellung, Berichtsdistribution und Berichtsaufnahme heranziehen. Diese stellen die Kernprozesse des Berichtswesens dar und wurden in Abschnitt 2.2.2 erörtert.

Exemplarisch wird in Abbildung 39 die schrittweise Verfeinerung des Kernprozesses der Informationsbeschaffung des Berichtswesens dargestellt. Den Anwendungshintergrund des gewählten Beispiels stellt die Erstellung eines Berichts dar, der am Monatsende die wichtigsten Kennzahlen zur Performance der Konzerntöchter beinhaltet. Die Bereitstellung dieser Informationen erfolgt unter Einsatz eines DWH-gestützten Berichtssystems. ${ }^{710}$

In Abbildung 39 ist ersichtlich, dass sich der Kernprozess der Informationsbeschaffung auf der zweiten Prozessebene in die Teilprozesse der Informationsidentifikation und Informationsaufbereitung gliedert. Während die Informationsidentifikation darauf ausgerichtet ist, sämtliche im Hinblick auf die Berichtsanforderungen erforderlichen Informationsquellen und die von diesen jeweils vorgehaltenen Informationen zu bestimmen, fokussiert die Informationsaufbereitung alle Verarbeitungsanweisungen, welche die identifizierten Informationen in einen auswertbaren Zustand versetzen.

$\mathrm{Da}$ in dem hier betrachteten Anwendungsfall eine DWH-Anwendung für das Reporting zum Einsatz kommen soll, lässt sich der Prozess der Informationsaufbereitung in die Teilprozesse Extraktion, Transformation und Laden aufspalten. ${ }^{711}$ Der Teilprozess der Extraktion sorgt für die Selektion und Bereitstellung der Quelldaten, die für die Kennzahlenermittlung verwendet werden. Da diese

709 MÜLLER führt in diesem Zusammenhang aus, dass eine Geschäftsprozessanalyse immer einen Geschäftsprozess voraussetzt. Vgl. Müller (2007b), S. 149. Darüber hinaus stellt der Autor fest, dass neben der Serviceidentifikation die Analyse und Beschreibung der Informationen und Informationsflüsse für die Gestaltung von Services von wesentlicher Bedeutung ist. Vgl. Müller (2007b), S. 145.

710 Siehe hierzu die Ausführungen in Abschnitt 2.2.1.1.

711 Zum ETL-Prozess vgl. die Ausführungen in Abschnitt 2.2.1.1. 
Daten typischerweise in heterogener Form und in verschiedenen Vorsystemen vorliegen, ist sicherzustellen, dass sie aus den identifizierten Quellen vollständig extrahiert und allen relevanten weiteren Teilprozessen zugeführt werden.

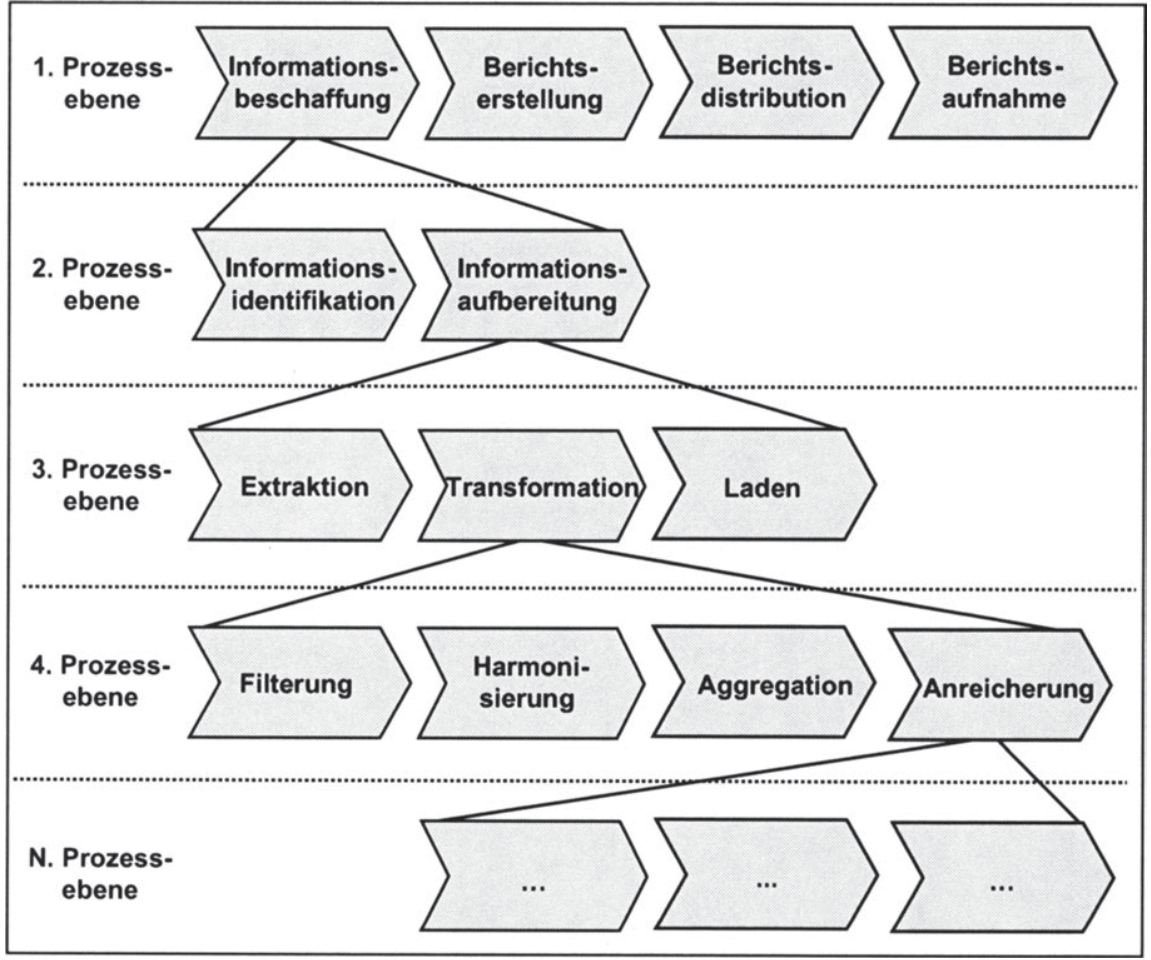

\section{Abbildung 39: Exemplarische Zerlegung des Berichtsprozesses Informationsbe- schaffung}

Der Teilprozess der Transformation fokussiert die Umwandlung der Quelldaten in das DWH-Zielformat. Als weitere Teilprozesse lassen sich die Filterung, Harmonisierung, Aggregation und Anreicherung identifizieren. Die Filterung der Daten gewährleistet, dass nur die für die Berichtserstellung sowie für eine eventuelle multidimensionale Analyse notwendigen Daten in das DWH des Berichtssystems gelangen. Der sich hieran anschließende Teilprozess der Harmonisierung befreit die Daten von syntaktischen und semantischen Mängeln. Zudem werden Kodierungen, Synonyme und Homonyme aufeinander abgestimmt sowie unterschiedliche Begriffsabgrenzungen vereinheitlicht. Der darauf einsetzende Teilprozess der Transformation hat zum Ziel, den nun konsistenten, aber 
in der niedrigsten Granularitätsstufe vorliegenden Datenbestand in Bezug auf individuell festzulegende Kriterien zu aggregieren, um eine Leistungssteigerung der nachfolgenden Analyse zu bewirken. Die Anreicherung schließt den Transformationsprozess ab. Hier findet eine Erweiterung der Daten um funktionale Aspekte statt, indem beispielsweise Berechnungen durchgeführt werden, die für die spätere Berichtserstellung notwendig sind.

Beim Ladeprozess, der dem Transformationsprozess folgt, werden die extrahierten und transformierten Daten in das DWH geladen und für eine spätere Nutzung dauerhaft abgelegt. ${ }^{712}$ Wie in der Abbildung $39 \mathrm{zu}$ erkennen ist, lassen sich die bestehenden Teilprozesse auf einer tieferen Ebene in weiteren Teilprozessen verfeinern. In diesem Zusammenhang muss entschieden werden, inwieweit eine weitere Zerlegung eines Teilprozesses fachlich sinnvoll ist.

\subsection{Einsatz von Modellierungsmethoden}

Für die strukturierte Darstellung der Prozessbeschreibungen sind geeignete Modellierungsmethoden einzusetzen. ${ }^{713}$ Dabei lassen sich zur Modellierung von Prozessen grafische Werkzeuge mit einer spezifischen Modellunterstützung verwenden. ${ }^{714}$ Angestrebt wird eine Standardisierung von Prozessen mit Hilfe grafischer Objekte, die von der jeweiligen Modellierungssprache zur Verfügung gestellt werden. Im deutschsprachigen Raum hat sich zur Konstruktion von Geschäftsprozessmodellen auf fachkonzeptioneller Ebene aufgrund ihres Anwendungsbezugs und einer umfassenden Tool-Unterstützung die ereignisgesteuerte Prozesskette (EPK) durchgesetzt. ${ }^{715}$ Als Modellierungskonzept hat sich die EPK sowohl in der Wissenschaft als auch in der Praxis etabliert. 716

712 Um eine zeitnahe Bereitstellung der Daten zu gewährleisten, ist dabei der Frage nachzugehen, in welchen Zeitintervallen die Daten in das DWH geladen werden müssen, um eine ausreichende Aktualität und Qualität zu ermöglichen. Diese Entscheidung ist vor dem Hintergrund der zur Verfügung stehenden Hardwareausstattung und eingesetzten Software zu treffen. Generell muss in diesem Zusammenhang abgewägt werden zwischen der gewünschten Qualität und Dauer einer Datenanalyse bzw. Berichtserstellung sowie den für die Aufbereitung der multidimensional modellierten Daten benötigten Systemressourcen. Ziel sollte es sein, ein adäquates Verhältnis von Leistungseinbußen und Erkenntnisgewinn zu gewährleisten.

713 Vgl. Beverungen/Knackstedt/Müller (2008), S. 223.

714 Vgl. Oehler (2006), S. 104.

715 Vgl. Nüttgens/Rump (2002), S. 64. Zur Prozessmodellierung lassen sich auch die Unified Modeling Language (UML) oder Petri-Netze einsetzen. Daneben ist auch der Einsatz von Aktivitätsdiagrammen möglich. Zur Klassifizierung verschiedener Methoden der Prozessmodellierung vgl. Gehring/Gadatsch (1999), S. 10f.; Specht/Weisbecker/ Spath (2006), S. 11.

716 Vgl. Seidlmeier/Scherfler (2007), S. 95. 
Mit einer EPK lässt sich der Ablauf eines Geschäftsprozesses logisch und zeitlich beschreiben.717 Die EPK-Darstellung ist ein bipartiter gerichteter Graph, in dem sich zwei Mengen von Knoten abwechseln: Ereignisse und Funktionen. Auf diese Weise wird eine logische 1:1-Zuordnung erreicht, die ausdrückt, welches Ereignis als Eingabe von welcher Funktion behandelt werden soll und welches Ereignis als Ausgabe einer Funktion entsteht. Die Verknüpfungslinien zwischen den Knoten sind gerichtet, da die Funktionen nach den Ereignissen ausgeführt werden bzw. die nächsten Ereignisse nach den Funktionen entstehen. Somit entsteht eine zeitliche und alternierende Abfolge von Ereignissen und Funktionen.

Die EPK bietet die Syntaxregeln UND, ODER und EXKLUSIV-ODER zur Verknüpfung und Verzweigung an. Vor einer Funktion lassen sich Ereignisse verknüpfen, die eine Bedingung zur Ausführung der nächsten Funktion darstellen, die selber wiederum mehrere Ereignisse erzeugen kann. Ebenso kann ein Ereignis zur Verzweigung in mehrere Funktionen führen, deren Ergebnisse wiederum zu einem Ereignis verbunden werden. Schließlich gibt es zur Partitionierung des Graphen zudem einen Prozesswegweiser, der mehrere Elemente zusammenfasst. Der Prozesswegweiser kann geöffnet werden, um die darin enthaltenen Schritte anzuzeigen.

Die ursprünglichen Möglichkeiten der EPK lassen sich um Elemente erweitern, mit denen den Funktionen auch die Organisationseinheiten, in denen die Prozesse ausgeführt werden, sowie die von der Ausführung betroffenen Dokumente zugeordnet werden. Diese und andere Elemente sowie weitere Regeln, wie z. B. solche zur Darstellung von Schleifen, sind in der erweiterten EPK (eEPK) enthalten. ${ }^{718}$ Aktuelle Ansätze versuchen, die Ausführung von Funktionen durch Services in der Modellbeschreibung zu berücksichtigen. ${ }^{719}$ Dafür werden weitere Elemente zur Beschreibung von Services (synchron, asynchron) und Ausnahmen-Ereignisse (Timeout, Nachricht nicht erhalten) unter dem Titel serviceorientierte EPK (sEPK) eingeführt, die sich in die Graphen aus den bekannten Elementen nahtlos integrieren lassen.

\subsubsection{Einbindung einer Lebenszyklusbetrachtung der Berichtsservices als übergeordneter Prozess}

Für die Gestaltung eines SOBP reicht die Anwendung einer geeigneten Methodik, mit der die Realisation der geforderten Funktionalität durch den zu kon-

717 Baumgartner/Ebert/Schleider (o. J.), S. 5.

718 Vgl. Scheer/Thomas (2005), S. 1069ff.

719 Vgl. Huth/Wieland (2008), S. 6. 
zipierenden Berichtsservice angestrebt wird, allein allerdings nicht aus. ${ }^{720}$ Gleichzeitig muss sichergestellt werden, dass die Qualität der eingesetzten Berichtsservices das Mindestmaß erreicht, das von der SOA-betreibenden Unternehmung vorgegeben ist. Wird dem Grundgedanken einer SOA gefolgt, applikations- und/oder unternehmungsübergreifende Geschäftsprozesse mittels verknüpfter Dienste effizient auszuführen, so muss die Nutzung der Berichtsservices über deren gesamte „Lebensdauer“ mit Hilfe geeigneter betriebswirtschaftlicher Instrumente begleitet werden. Dies betrifft auch unternehmungsexterne Berichtsservices, die in einem SOBP eingebunden werden sollen. Die Betrachtung der Lebensdauer eines Berichtsservices ist von Bedeutung, da sich Berichtsprozesse beispielsweise im Zuge neuer gesetzlicher Anforderungen oder einer Veränderung der Konzernstruktur verändern. Werden zudem unternehmungsexterne Berichtsservices eingesetzt, um beispielsweise Währungen umzurechnen oder Aktienkurse zu aktualisieren, erscheint die Bewertung der Qualität der Services umso wichtiger.

Als geeigneter Ansatz lässt sich für diese Zwecke eine Lebenszyklusbetrachtung durchführen. Eine solche Betrachtung wird im aktuellen Abschnitt angestrebt. Die Ausführungen stützen sich auf einen Beitrag von BERBNER ET AL., 721 in welchem die Autoren zur Durchführung einer Lebenszyklusbetrachtung von Web Services acht Phasen von einander abgrenzen. ${ }^{722}$ Diese sind in Abbildung 40 verdeutlicht.

720 Die Designprinzipien der Serviceorientierung stellen in diesem Zusammenhang die zentralen Konzepte für die Einbindung der Services in ein Verzeichnis, für die Orchestrierung sowie Nutzung der Dienste dar.

721 Vgl. Berbner et al. (2005), S. 269ff.

722 Ein Phasenmodell zur Lebenszyklusbetrachtung findet sich unter anderem auch bei Burghardt (2004), S. 18f. Da die Lebenszyklusbetrachtung bei BERBNER ET AL. detaillierter ist, wird diesem Modell der Vorzug gegeben. 


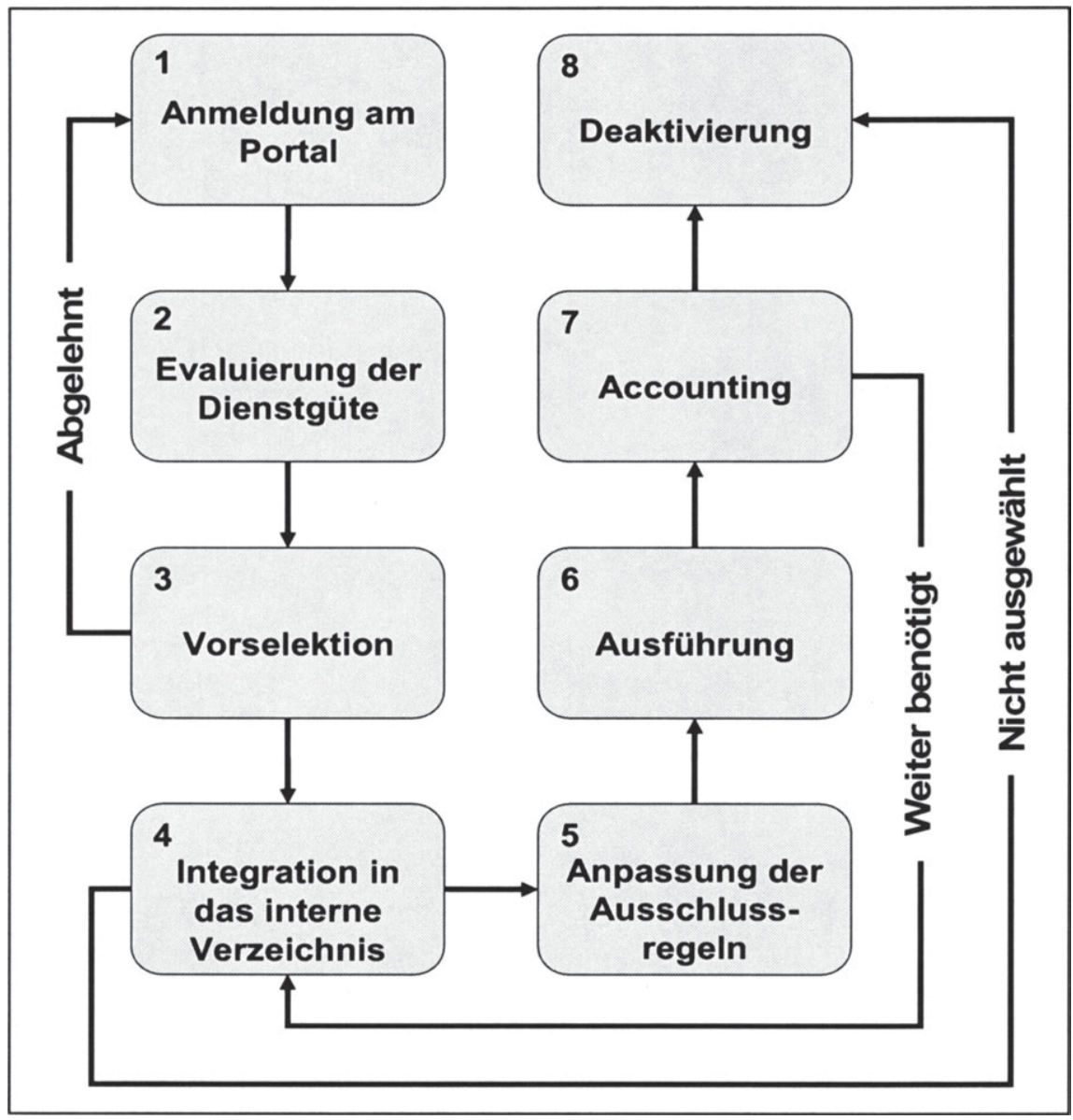

Abbildung 40: Lebenszyklus eines Berichtsservices

\section{Phase: Anmeldung am Portal des Nutzers}

Die erste Phase des Lebenszyklus eines Berichtsservices betrifft nur diejenigen Dienste, die von einem unternehmungsexternen Anbieter zur Verfügung gestellt werden. Bevor ein unternehmungsexterner Berichtsservice genutzt werden kann, muss der betreffende Service von den potenziellen Nutzern begutachtet und zur Nutzung freigegeben werden. Hierfür ist es notwendig, dass sich der 
Anbieter des Dienstes zunächst am Portal des Nutzers registriert. ${ }^{723}$ Bei der Registrierung sind alle erforderlichen Informationen zur Kontaktaufnahme wie Anschrift oder Ansprechpartner anzugeben. Im Anschluss hat der ServiceProvider die Möglichkeit, den von ihm angebotenen Berichtsservice am Portal des Nutzers anzumelden. Eine Anmeldung gilt als abgeschlossen, wenn der Service-Provider verbindlich alle Dienstgüteeigenschaften in Form eines Service Level Agreements (SLA) zu dem von ihm angebotenen Dienst zur Verfügung stellt. 724

\section{Phase: Evaluierung der Dienstgüte}

Nachdem der Berichtsservice mit zugehörigem SLA am Portal angemeldet wurde, ist im zweiten Schritt über die Qualität des angebotenen Services zu entscheiden. Die Bewertung der in Frage kommenden Services wird von den potenziellen Nutzern durchgeführt. Die Evaluation der Dienstgüte der Berichtsservices ist erforderlich, damit die angebotenen Dienste verschiedener Provider auf Basis ihrer Dienstgüteeigenschaften miteinander verglichen werden können. Mit Hilfe von Gewichtungsfaktoren lassen sich Präferenzen und einsatzspezifische Besonderheiten bei der Bewertung der Berichtsservices berücksichtigen. In gleicher Weise ist mit allen Services zu verfahren, die eigenentwickelt werden und dem Serviceportfolio zugeführt werden sollen.

\section{Phase: Vorselektion}

Die Phase der Vorselektion wird mit dem Ziel durchgeführt, auf Basis der Ergebnisse der zweiten Phase mit Hilfe von Ausschlussregeln zu entscheiden, welche Berichtsservices in das interne Verzeichnis aufgenommen werden. Grundsätzlich lassen sich drei Varianten einer Ausschlussregel definieren. Bei der ersten Variante werden alle Kriterien eines Dienstes bei der Definition einer Ausschlussregel einbezogen. In diesem Fall ist es möglich, Schwellenwerte vorzugeben, die ein Berichtsdienst erfüllen muss, um in das interne Serviceverzeichnis der SOA-betreibenden Unternehmung aufgenommen zu werden.

Im Unterschied zur ersten Variante setzt sich eine Ausschlussregel bei der zweiten Variante nur aus ausgewählten Kriterien zusammen. Schwellenwerte lassen sich wie bei der ersten Variante ebenfalls einsetzen, um eine Ausschlussregel zu definieren. Eine Verknüpfung der Teilkriterien ist ebenfalls möglich.

723 An dieser Stelle wird angenommen, dass der Bereitschaft des Service-Providers, einen Dienst anzumelden, eine Anbahnungsphase vorausgegangen ist. Ein Service-Provider kann beispielsweise infolge einer öffentlichen Ausschreibung seinen Berichtsservice anbieten. Alternativ ist der Fall denkbar, dass die SOA-betreibende Unternehmung ihrerseits aktiv in öffentlichen Verzeichnissen nach geeigneten Diensten sucht und die betreffenden Anbieter zur Anmeldung ihrer Services einlädt.

Zum Begriff des SLA vgl. Abschnitt 5.2.3.4. 
Darüber hinaus lässt sich neben einer absoluten Bewertung der Berichtsservices auch eine relative Betrachtung durchführen. Die letzte Bewertungsvariante liegt z. B. vor, wenn nur Dienste selektiert werden dürfen, die sich unter den zehn „besten“ Services befinden.

Als dritte Variante lassen sich Berichtsservices unabhängig von einer quantitativen Bewertung ausschließen. Ein solcher Fall ist gegeben, wenn die SOAbetreibende Unternehmung beispielsweise aus strategischen Erwägungen grundsätzlich nicht bereit ist, Dienste von einem bestimmten externen Provider zu verwenden.

Die unternehmungsinternen und -externen Provider der nicht berücksichtigten Berichtsservices können über Gründe für die Ablehnung benachrichtigt werden. ${ }^{725}$ Die unternehmungsexternen Provider haben im Anschluss die Möglichkeit, die Qualität des von ihnen angebotenen Services zu verbessern und den Dienst erneut am Portal anzumelden. Wird hingegen ein unternehmungsinterner Dienst abgelehnt, so besteht auch hier die Möglichkeit, Anpassungen an dem betreffenden Service vorzunehmen, um die Dienstgüte zu steigern.

\section{Phase: Integration in das interne Verzeichnis}

Die Aufnahme eines Berichtsservices in das interne Verzeichnis erfolgt bei allen Diensten, die vorselektiert wurden (3. Phase). Nach der Einbindung der Services in das interne Verzeichnis stehen die Dienste zur Nutzung zur Verfügung. ${ }^{726}$ Hier wird der Fall eintreten, dass einige Berichtsservices im Laufe ihrer Lebensdauer nicht oder nur selten ausgeführt werden, so dass nach einiger Zeit diese Services deaktiviert werden können (8. Phase).

\section{Phase: Anpassung der Ausschlussregeln}

In den Ausführungen zur zweiten und dritten Phase wurde deutlich, dass diejenigen Services zur Anwendung kommen sollten, deren Dienstgüteeigenschaften im Vergleich zu konkurrierenden Serviceangeboten gleich sind oder diese übertreffen. Bei der Selektion eines Berichtsservices zur Laufzeit lassen sich ergänzend zu den bereits vorhandenen Ausschlussregeln weitere Nebenbedingungen definieren, die bei der Aufnahme eines Dienstes in das Verzeichnis zu berücksichtigen sind. Der Einsatz solcher Nebenbedingungen hängt von den Anforderungen sowie der Bedeutung des auszuführenden Berichtsprozesses ab. Ist abzusehen, dass ein Berichtsservice besonders häufig ausgeführt werden muss,

725 Ebenso können die Serviceanbieter informiert werden, wenn der Dienst nach einer gewissen Nutzungszeit aus dem Verzeichnis genommen wird. Vgl. Burghardt (2004), S. 19.

726 Die Integration der Services in das Verzeichnis der SOA-betreibenden Unternehmung wird auch als Deployment bezeichnet. Vgl. hierzu Burghardt (2004), S. 18. 
lassen sich an diesen Service weitere bzw. höhere Anforderungen definieren, die erfüllt sein müssen, damit der jeweilige Dienst zum Einsatz kommt. ${ }^{727}$

\section{Phase: Ausführung}

Nachdem die Dienstgüte des jeweiligen Berichtsservices die erforderlichen Kriterien erfüllt hat und der Dienst in das interne Verzeichnis integriert wurde, steht der betreffende Service zur Nutzung zur Verfügung. Die Berichtsservices werden dynamisch zur Laufzeit zu einem SOBP gekoppelt und ausgeführt. Innerhalb des Lebenszyklus eines Berichtsservices nimmt die Phase der Ausführung die längste Zeit ein.

\section{Phase: Accounting}

Das Ziel dieser Phase ist die verursachungsgerechte Zurechnung der Nutzung eines Berichtsservices. Sowohl für den Serviceanbieter als auch für den -nachfrager ist die Be- und Zurechnung der Serviceausführung von Bedeutung. Kommen Berichtsservices zum Einsatz, die von einem unternehmungsexternen Provider gegen Entgelt bereitgestellt werden, ist Sorge zu tragen, dass die fälligen Gebühren für die Inanspruchnahme des Services an den Anbieter fristgerecht entrichtet werden. Den Servicenehmer unterstützt das Accounting bei der verursachungsgerechten Zuordnung der für die Inanspruchnahme der Berichtsservices anfallenden Kosten. Die Kosten sind den internen Organisationseinheiten zuzurechnen, welche die betreffenden Dienste nutzen.

In der Phase des Accounting ist ferner darüber zu entscheiden, ob der betreffende Berichtsservice weiterhin genutzt oder aus dem Servicebestand entfernt werden soll.

\section{Phase: Deaktivierung}

Die Phase der Servicedeaktivierung bildet den Abschluss des Lebenszyklus eines Berichtsservices. Ein Berichtsservice muss deaktiviert werden, wenn die von ihm bereitgestellte Funktionalität nicht mehr benötigt wird. In diesem Fall ist der betreffende Dienst aus dem internen Verzeichnis zu entfernen. Ein weiterer Grund für die Deaktivierung eines Berichtsservices ist gegeben, wenn der Anbieter des Dienstes den entsprechenden Service nicht mehr zur Nutzung zur Verfügung stellen kann oder möchte.

727 Derartige Anforderungen betreffen z. B. alle in Abschnitt 5.2.3.4.1 erläuterten technischen Dienstgüteeigenschaften. 


\subsubsection{Prozessmodellbasierte Serviceidentifikation}

Bevor Berichtsservices fachlich gestaltet und zu einem SOBP verknüpft werden können, ist es notwendig, die potenziellen Services aus einem Prozessmodell heraus $\mathrm{zu}$ identifizieren. ${ }^{728}$ In diesem Zusammenhang ist $\mathrm{zu}$ konstatieren, dass sich bisher kein einheitliches methodisches Vorgehen zur Serviceidentifikation durchgesetzt hat. ${ }^{729}$ Das Ziel der nachfolgenden Ausführungen richtet sich daher darauf, eine geeignete Methodik zu entwickeln, die sich zur Identifikation von Berichtsservices einsetzen lässt.

In Abschnitt 5.2.2.1 wird zunächst die Bedeutung der Wiederverwendung von Services beleuchtet. Die Wiederverwendbarkeit von Services ist das zentrale Ziel der Serviceidentifikation. In diesem Zusammenhang wird der Begriff der Granularität eingeführt, der für die weiteren Ausführungen von besonderer Relevanz ist. Da das in dieser Arbeit konzipierte Vorgehensmodell dem Meet-inthe-middle-Ansatz entspricht, 730 ist zu bemerken, dass bei der Serviceidentifikation die Top-down- und Bottom-up-gestützte Herangehensweise kombiniert und integrativ zum Einsatz kommen. Vor diesem Hintergrund widmet sich Abschnitt 5.2.2.2 zunächst der Top-down-gestützten Serviceidentifikation, während in Abschnitt 5.2.2.3 die Bottom-up-gestützte Herangehensweise im Vordergrund steht.

\subsubsection{Wiederverwendung als Ziel der prozessmodellbasierten Service- identifikation}

Die Modellierung des gesamten Berichtsprozesses hat zum Ziel, Berichtsservices zu identifizieren, die sich durch einen hohen Wiederverwendungsgrad auszeichnen. Dabei wird ein Service als wiederverwendbar bezeichnet, wenn er sich nicht nur innerhalb eines Anwendungsgebiets, sondern auch über Fachbereichsgrenzen hinweg für verschiedene Problemstellungen sowie in einer Vielzahl von heterogenen Anwendungen nutzen lässt. ${ }^{731}$ Mit der Wiederverwendbarkeit von Diensten wird ein Ziel verfolgt, das generell an Serviceorientierte Anwendungen gerichtet ist. Mit Blick auf die Gestaltung und Ausführung eines SOBP liegt eine hohe Wiederverwendbarkeit eines Berichtsservices dann vor, wenn der Dienst in verschiedenen SOBP einsetzbar ist. 732

728 Auf die generelle Bedeutung, bei der Konzeption einer SOA ein wichtiges Augenmerk auf die Identifikation der Services bzw. Strukturierung der Gesamtfunktionalität einer Anwendung in wohldefinierte Dienste zu legen, verweisen auch HÖß ET AL. und KLOSE/ KNACKSTEDT. Vgl. Höß et al. (2007), S. 40; Klose/Knackstedt (2007), S. 47.

Vgl. Klose/Knackstedt (2007), S. 47; Müller (2007b), S. 145.

Vgl. hierzu die Ausführungen in Abschnitt 5.1.4

Vgl. Gimnich (2007), S. 68; Winkler (2007), S. 258.

732 Vgl. Winkler (2007), S. 258. 
Die Wiederverwendung von Services ist von Bedeutung, da sich auf diese Weise signifikante Einsparpotenziale für die SOA-betreibende Unternehmung ergeben. Das Einsparpotenzial, das aus der Wiederverwendung vorhandener Software resultiert, beläuft sich nach der Einschätzung von BALZERT beispielsweise auf $1 / 4$ der Entwicklungszeit sowie der dafür eingesetzten Ressourcen. ${ }^{733}$ Ein wichtiger Faktor für die Wiederverwendbarkeit eines Services ist seine Granularität. Die Granularität steht für den Umfang und die Art der Funktionen, die durch einen Service ausgeführt werden. ${ }^{734}$ Services mit einer hohen Granularität verarbeiten eine Vielzahl von Datenobjekten sowie vereinen verschiedene Aktionen, die sequenziell ausgeführt werden. Demgegenüber führen Dienste mit einer niedrigen Granularität nur einzelne oder wenige Aktionen aus.

Die Bedeutung der Servicegranularität lässt sich anhand der Auswirkungen verdeutlichen, die sich einstellen, wenn Services nicht in der geforderten Granularität bereitgestellt werden. Werden Berichtsdienste mit geringer Granularität eingesetzt, ist das Ergebnis eine große Anzahl von Services. Dies hat zur Folge, dass die Verwaltung der Dienste erschwert sowie die Suche nach dem richtigen Service aufwendiger wird. Werden hingegen Reportingservices mit einer groben Granularität bevorzugt, besteht die Gefahr, dass die Dienste aufgrund ihres (zu) groben Funktionsumfangs nicht die Fähigkeit besitzen, in anderen Berichtsprozessen eingesetzt zu werden. Der Vorteil einer Wiederverwendung und die sich daraus ergebenden Potenziale zur Flexibilisierung der Geschäftsprozesse werden im Fall grobgranularer Services demzufolge gemindert. 735

Um Services mit einer hohen Wiederverwendbarkeit einzusetzen, muss sichergestellt werden, dass sich die Dienste ohne größeren Aufwand in die zu unterstützenden Prozesse einbinden lassen. ${ }^{736}$ Diese Anforderung wird vom Designprinzip der „Abstraktion von der Implementierungslogik“ berücksichtigt. ${ }^{737}$ Die Einhaltung dieses Designprinzips hat zur Folge, dass Aktionen, die gemeinsam auftreten, in einem Service gekapselt werden können. Die Kapselung derartiger Aktionen bietet den Vorteil, dass sich die Anzahl der Services verringern lässt.

Vgl. Balzert (2001), S. 79. An dieser Stelle ist darauf hinzuweisen, dass diese von BALZERT formulierte Regel allgemeingültigen Charakter besitzt, d. h. nicht auf die explizite Wiederverwendung von Services abzielt, sondern sich auf die generellen Vorteile wiederverwendbarer Software bezieht.

Vgl. Erl (2005), S. 209 und 302; Fiege/Stelzer (2007), S. 911.

Vgl. Buxmann/Hess/Widjaja (2007), S. 1318.

Vgl. hierzu Winkler (2007), S. 258.

Vgl. Abschnitt 3.3.2.1. 


\subsubsection{Top-down-gestützte Serviceidentifikation}

Der folgende Abschnitt zeigt, wie sich eine Top-down-gestützte Identifikation der Berichtsservices durchführen lässt. Ausgangspunkt sind die zugrunde liegenden Berichtsprozessmodelle. Ziel der Berichtsidentifikation ist es, aus den Berichtsprozessmodellen heraus Aktivitäten zu ermitteln, die als potenzielle Berichtsservices in Frage kommen. Die dabei anfallenden Schritte sind Gegenstand des Abschnitts 5.2.2.2.1, während zwei Beispiele eines potenziellen Berichtsservices im anschließenden Abschnitt 5.2.2.2.2 präsentiert werden.

\subsection{Vorgehen}

Grundsätzlich lässt sich die Top-down-gestützte Serviceidentifikation anhand von drei Schritten durchführen. Die hierbei relevanten Aktivitäten werden nun verdeutlicht.

\section{Schritt 1: Ermittlung mehrfach auftretender und/oder berichtsprozess- übergreifender Aktivitäten}

Nach der Modellierung der zu unterstützenden Berichtsprozesse wird eine Aufbereitung der Prozessmodelle dann erforderlich, wenn sich in ihnen gleichartige Aktivitäten bzw. Aktionen identifizieren lassen,738 die entweder innerhalb eines Berichtsprozesses mehrfach oder aber in verschiedenen Berichtsprozessen auftauchen. ${ }^{739}$ Derartige Aktivitäten sind potenzielle Berichtsservices, da sie aufgrund ihres mehrfachen Auftretens eine hohe Wiederverwendung in Aussicht stellen. Wie anhand des Aktivitätsdiagramms von Abbildung $41 \mathrm{zu}$ erkennen ist, stellt die Aktivität 4.1 einen solchen Berichtsservicekandidaten dar, da diese Aktion von den übergeordneten Aktivitäten 2.3, 3.1 und 3.2 verwendet wird. ${ }^{740}$

Um redundante Aktivitäten zu ermitteln und zu minimieren, sind hierbei inhaltlich gleiche Aktivitäten auch einheitlich zu benennen. Wird keine einheitliche Semantik gewährleistet, erweist sich die Identifikation mehrmals modellierter Aktivitäten mit Blick auf die mögliche Komplexität von Berichtsprozessen als schwierig bzw. unmöglich. Durch die eindeutige Benennung mehrfach vorkommender Aktionen lässt sich sicherstellen, dass im Ergebnis Aktivitäten vorliegen, die eindeutig voneinander abgrenzbare Aufgaben in dem zugrunde liegenden Berichtsprozess repräsentieren. Ist dieser Zustand nicht gegeben, muss an dieser Stelle eine Aufbereitung der Berichtsprozessmodelle erfolgen.

738 Die Begriffe „Aktivität“ und „Aktion“ werden im Folgenden synonym verwendet.

739 Vgl. Winkler (2007), S. 259.

740 In dem exemplarischen Aktivitätsdiagramm von Abbildung 41 sind die Aktivitäten durchnummeriert, wobei die Zahl vor dem Punkt für die Ebene steht, auf der sich die jeweilige Aktivität befindet. 
Bei der Anpassung der Berichtsprozessmodelle ist dabei zu beachten, dass die Beziehungen zwischen den mehrmals in einem Prozess vorkommenden Aktionen und den nur einmal vorkommenden Aktivitäten erhalten bleiben. So kann die korrekte Ausführung der Berichtsprozesse gewährleistet werden

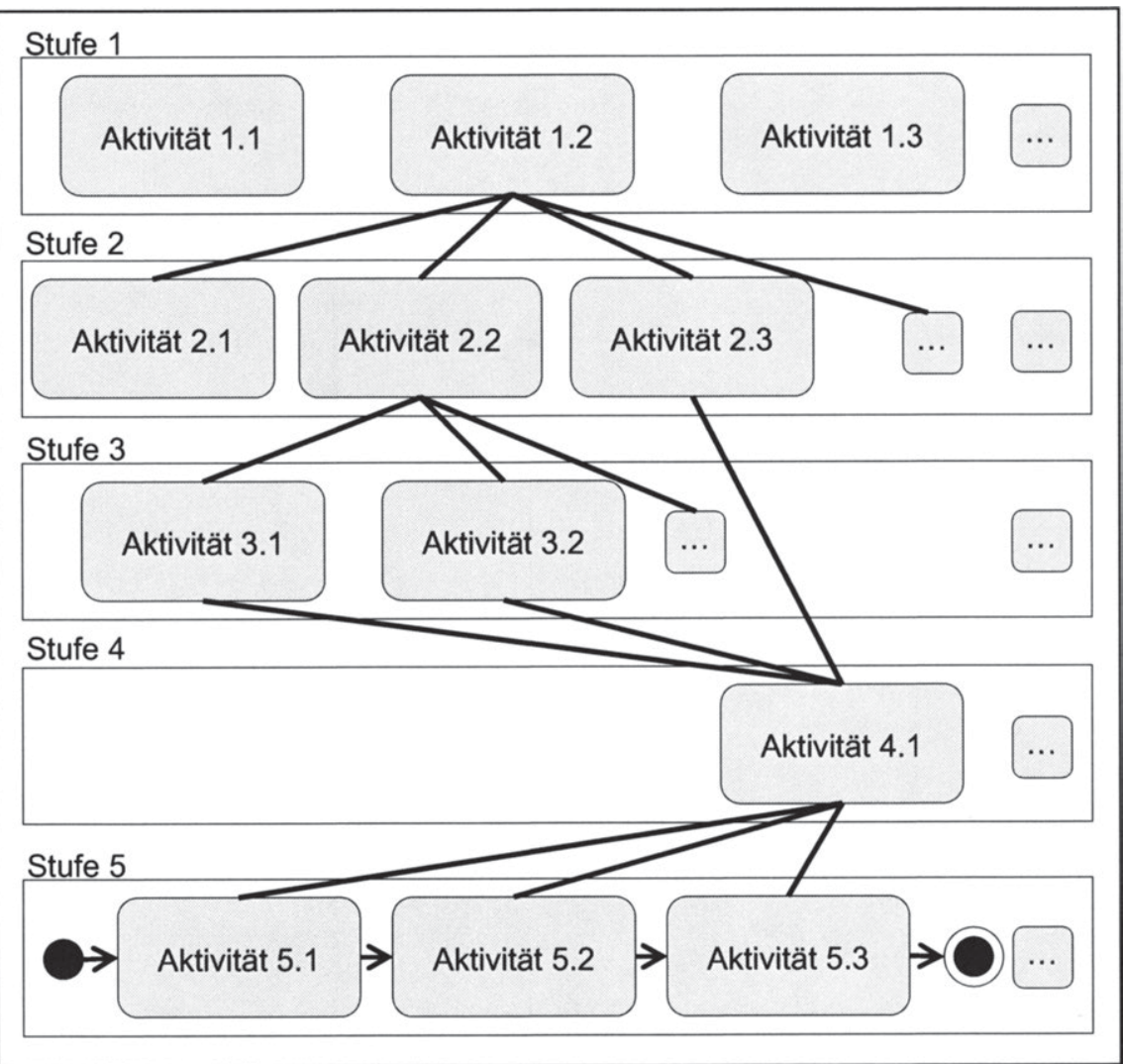

Abbildung 41: Beispiel eines aufbereiteten Aktivitätsdiagramms

\section{Schritt 2: Identifikation potenzieller Berichtsservices durch Verknüpfung zusammenhängender Berichtsprozessaktivitäten}

Nach der Aufbereitung der zugrunde liegenden Berichtsprozessmodelle erfolgt die Identifikation der potenziellen Berichtsservices, die in einem Berichtsprozess mehrfach und/oder in verschiedenen Berichtsprozessen auftreten. Das entscheidende Kriterium ist hierbei, alle Aktionen bzw. Aktivitäten zu einem Berichtsservice zusammenzufassen, die bei der Inanspruchnahme dieses Dienstes sequenziell ausgeführt werden. Die Identifikation derartiger Aktionen wird 
mit dem Ziel durchgeführt, alle Aktionen zu ermitteln, die sich zu einem Service kapseln lassen. In Abbildung 41 wird deutlich, dass diese Konstellation bei Aktion 4.1 vorliegt, da bei dieser Aktion die Basisaktivitäten 5.1 bis 5.3 hintereinander ausgeführt werden. ${ }^{741}$

\section{Schritt 3: Ermittlung der Häufigkeit der Serviceverwendung}

Nach der Identifikation der potenziellen Berichtsservices ist zu klären, wie häufig diese genutzt werden. Ziel dieses Schrittes ist es, den „Kandidatenkreis“ potenzieller Berichtsservices einzugrenzen. Die Häufigkeit der Servicenutzung lässt sich anhand von zwei Kriterien bestimmen. Zum einen ist zu ermitteln, wie oft ein Service innerhalb eines bestimmten Zeitraums aufgerufen wird (liegt eine häufige Nutzung vor, sind besonders hohe Anforderungen an die Verfügbarkeit des Dienstes zu formulieren). ${ }^{742}$ Zum anderen muss bekannt sein, von wie vielen weiteren Diensten ein Berichtsservice nachgefragt wird. Dies wiederum geht mit der Frage einher, in welchen weiteren SOBP der jeweilige Berichtsservice ausgeführt werden soll. Als potenzielle Servicenachfrager kommen hier sowohl unternehmungsinterne als auch -externe Servicenutzer in Frage.

In Anlehnung an WINKLER lässt sich in diesem Zusammenhang nicht präzisieren, was unter einer häufigen Serviceverwendung zu verstehen ist. ${ }^{743}$ Ein kritischer Wert, der als Mindestgrenze für die Häufigkeit einer Servicenutzung dient, ist daher von jeder SOA-betreibenden Unternehmung, $d$. h. von der ITsowie insbesondere von der Fachabteilung, welche die Nutzung des Berichtsservices anstrebt, individuell festzulegen.

\subsection{Beispiele für Berichtsservicekandidaten}

Die beiden folgenden Abschnitte 5.2.2.2.2.1 und 5.2.2.2.2.2 thematisieren Beispiele von Berichtsservicekandidaten, die sowohl in einem Berichtsprozess mehrmalig als auch berichtsprozessübergreifend zur Anwendung kommen können. Charakteristisch für diese Berichtsservices ist, dass sie aus verschiedenen Aktionen bestehen, die stets gemeinsam auftreten.

\subsection{Berechnung verknüpfter Kennzahlen im Rahmen des Prozesses „Berichtserstellung“}

Als Beispiel für eine Aktivität, die in verschiedenen SOBP zum Einsatz kommen kann, lässt sich die Berechnung von Kennzahlen bzw. Key-Performance-

741 Abschnitt 5.2.2.2.2 führt zwei Beispiele von Aktivitäten an, die als derartige Aktionen identifiziert werden können und sich damit als potenzielle Berichtsservices eignen.

742 Zur Dienstgüteeigenschaft der Verfügbarkeit vgl. Abschnitt 5.2.3.4.1.

743 Vgl. Winkler (2007), S. 261. 
Indikatoren (KPI) anführen. ${ }^{744}$ Kennzahlen besitzen für die Unternehmungssteuerung eine große Bedeutung. ${ }^{745}$ Mit Blick auf die in Abschnitt 2.2.2 vorgestellten Kernprozesse des Berichtswesens fällt die Ermittlung von Kennzahlen im Rahmen des Kernprozesses „Berichtserstellung“ an.

Kennzahlen dienen dazu, die Unternehmungsleitung und weitere Entscheidungsträger einer Unternehmung bei der kurzfristigen und langfristigen Planung, Steuerung und Kontrolle zu unterstützen. Sie stehen für Zahlen bzw. Messwerte, die messbare, betriebswirtschaftlich relevante Daten in komprimierter bzw. verdichteter Form zusammenfassen. ${ }^{746}$ Mit Hilfe von Kennzahlen lassen sich Zusammenhänge und Abhängigkeiten verdeutlichen sowie die Komplexität des dargestellten Sachverhalts reduzieren. ${ }^{747}$ Ferner kommen Kennzahlen zur Definition von Sollvorgaben und -bereichen, die sich beispielsweise auf bestimmte Produkte, Leistungen oder Geschäftsvorfälle beziehen, zum Einsatz. 748

Mit den absoluten Kennzahlen, den Verhältniszahlen und den Richtzahlen werden drei Kennzahlentypen unterschieden. ${ }^{749}$ Absolute Kennzahlen lassen sich den Betriebsdaten entnehmen. Sie können als Einzelzahlen, Summen, Differenzen oder Mittelwerte ausgeprägt sein. Umsatzerlöse, das Anlagevermögen sowie Forderungen aus Lieferungen und Leistungen sind Beispiele für eine absolute Kennzahl. Verhältniszahlen bzw. Relativzahlen setzen absolute Zahlen in Beziehung zueinander. Als Ausprägungen einer Verhältniszahl lassen sich Gliederungszahlen, Beziehungszahlen und Indexzahlen unterscheiden. Bei Gliederungszahlen wird ein Teilwert zu einem Gesamtwert in Beziehung gesetzt. Ein Beispiel hierfür ist die Eigenkapitalquote, die sich ergibt, wenn das Eigenkapital durch das Gesamtkapital dividiert wird. Eine Beziehungszahl wird berechnet, wenn absolute Zahlen, die in einem inhaltlichen Zusammenhang stehen, miteinander dividiert werden. Diese Konstellation liegt z. B. bei der Ermittlung des Deckungsgrads 1 vor. Die Berechnungsvorschrift lautet Deckungsgrad $1=($ Eigenkapital / Anlagevermögen $) * 100$. Indexzahlen, die auch als Messzahlen bezeichnet werden, zeichnen sich dadurch aus, dass gleichartige Zahlen, die zeitlich oder räumlich voneinander getrennt sind, zu einem Basis-

744 Nach SIEGWART haben Kennziffern im Gegensatz zu Kennzahlen eine grundsätzliche Bedeutung und gehen über betriebsspezifische Sachverhalte hinaus. Vgl. Siegwart (2002), S. 19. Im Gegensatz zu SIEGWART wird im Hinblick auf das Ziel dieses Abschnitts eine Unterscheidung der Termini Kennzahl und Kennziffer jedoch nicht weiterverfolgt.

745 Vgl. Weber et al. (2004), S. 98.

746 Vgl. Vollmuth/Zwettler (2006), S. 8.

747 Hierin liegt ein Unterschied zu den Informationen, die aus der Buchhaltung stammen.

Vgl. Vollmuth/Zwettler (2006), S. 9.

748 Vgl. Kronz (2005), S. 35.

749 Vgl. Zdrowomyslaw/Kasch (2002), S. 72. 
maß zusammengeführt werden. Richtzahlen als dritte Ausprägung einer Kennzahl lassen sich ermitteln, wenn Zahlen beispielsweise einer zu analysierenden Unternehmung mit branchenspezifischen Durchschnittszahlen in Beziehung gesetzt werden.

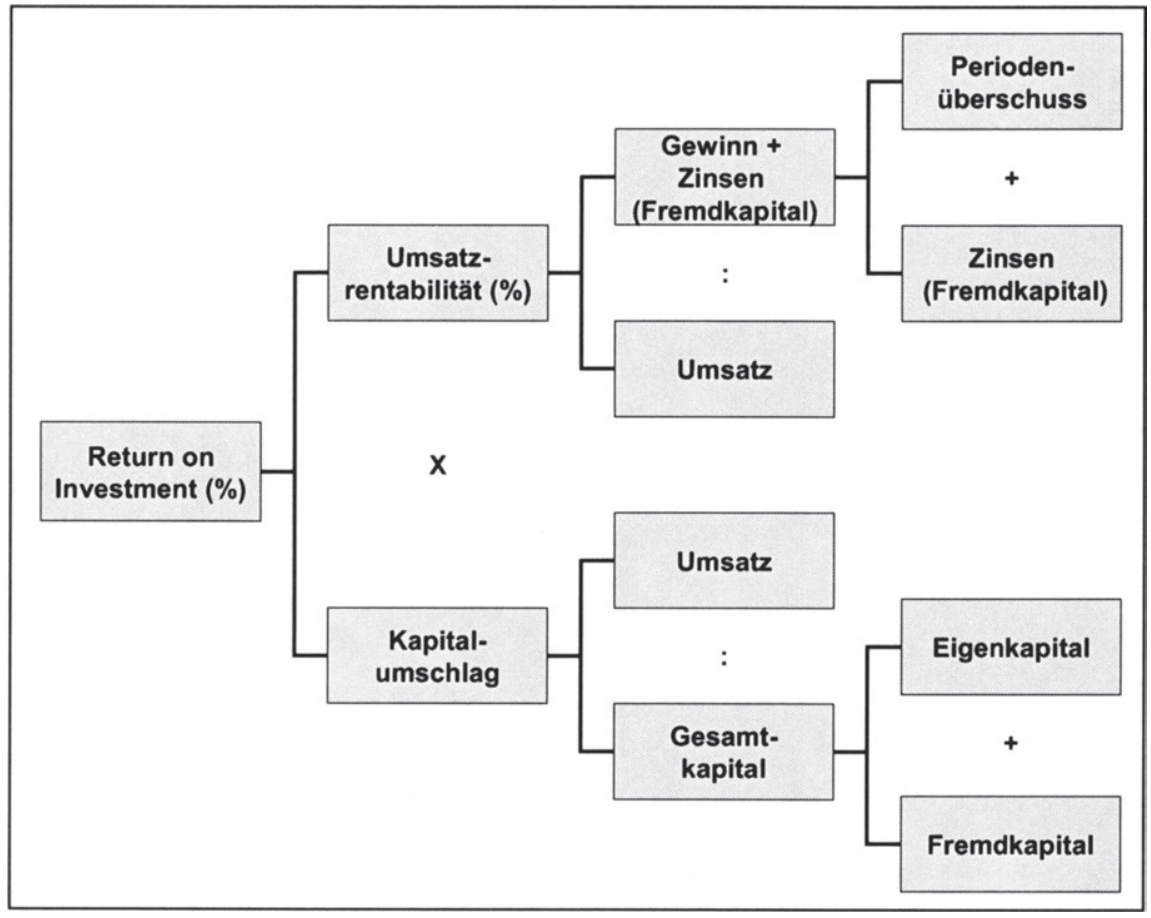

Abbildung 42: ROI-Baum nach dem DuPont-Schema 750

Mit Blick auf das in Abschnitt 5.2.2.2.1 vorgestellte Top-down-gestützte Vorgehen zur Identifikation gemeinsam auftretender Aktivitäten ist festzustellen, dass eine sequenzielle Ausführung mehrerer Aktionen z. B. bei der Ermittlung der Spitzenkennzahl eines Kennzahlensystems vorliegt. Ein Kennzahlensystem zeichnet sich dadurch aus, dass die Kennzahlen nicht isoliert voneinander be-

750 Vgl. Mertens/Billmeyer/Bradl (2003), S. 802. Der Kennzahlenbaum entspricht dem DuPont-Kennzahlensystem. 
trachtet, sondern in Beziehung zueinander gesetzt werden. ${ }^{751}$ Ein Beispiel für eine solche Spitzenkennzahl ist der ROI, der berechnet wird, um die Rendite des eingesetzten Kapitals zu ermitteln. Abbildung 42 verdeutlicht, aus welchen Teilkennzahlen sich der ROI zusammensetzt.

Wie Abbildung $42 \mathrm{zu}$ entnehmen ist, werden zur Berechnung des ROI verschiedene Teilkennzahlen in mathematische Beziehungen zueinander gesetzt. ${ }^{752}$ Aus der Perspektive der Serviceorientierung lassen sich die einzelnen Berechnungen der Teilkennzahlen als separate Aktivitäten auffassen, die abgestimmt aufeinander ausgeführt werden müssen, um die Spitzenkennzahl zu berechnen. Folglich lassen sie sich zu einem Berichtsservice kapseln, der die Berechnung des ROI zum Ziel hat.

\subsection{XBRL-Validierung von Jahresabschlussdaten im Rahmen der Prozesse „Informationsbeschaffung“ und „Berichtsdistribution“"}

Als weiteres Beispiel für einen Berichtsservice, der innerhalb eines Berichtsprozesses mehrfach oder in verschiedenen SOBP verwendet werden kann, ist ein Dienst zu nennen, der die Validierung von XBRL-Instanzdokumenten übernimmt. Ein solcher Service kann beispielsweise die Aufgabe erfüllen, Bilanzen, die nach einem bestimmten Rechnungslegungsstandard erstellt wurden und als XBRL-Instanzdokument vorliegen mit der XBRL-Taxonomie zu validieren, welche den zugrunde liegenden Rechnungslegungsstandard abbildet, nach dem das Instanzdokument erstellt wurde.

Eine derartige Validierung von Bilanzinformationen ist empfehlenswert, um die semantische Korrektheit der zu verarbeitenden Informationen zu garantieren. Ein Anwendungsfall ergibt sich bei der Konzernkonsolidierung, bei der die Bilanzen der Töchterunternehmungen zunächst beschafft werden müssen. Die Bilanzen der Töchter lassen sich einerseits als Dokumente in einem proprietären Datenformat anliefern. Alternativ können die Tochterbilanzen als XBRLInstanzen zur Verfügung gestellt werden. Um sicher zu gehen, dass die Inhalte der Instanzen dem zugrunde liegenden Rechnungslegungsstandard genügen, sind die Dokumente mit der entsprechenden XBRL-Taxonomie zu validieren. Die beim Validierungsprozess anfallenden Aktionen lassen sich zu einem Be-

751 Damit bilden Kennzahlensysteme die Grundlage für Berichtsprozesse, die auf das Benchmarking ausgerichtet sind. Als Benchmarking wird der Vergleich mit anderen Wirtschafts- oder Verwaltungssubjekten auf Basis identisch erhobener Messgrößen verstanden. Zum Vergleich wird der Beste einer Branche herangezogen. Durch den Einsatz von Kennzahlensystemen lässt sich nicht nur Wissen in komprimierter Form über die abgebildete Realität des betrachteten Subjekts abbilden, sondern lassen sich auch Veränderungsprozesse anstoßen.

752 Wie in der Abbildung 42 deutlich wird, ergibt sich der ROI, indem die Umsatzrentabilität mit dem Kapitalumschlag multipliziert wird, während sich dieser durch die Division des Umsatzes mit dem Gesamtkapital ermitteln lässt. 
richtsservice kapseln, der bei Bedarf in einem Berichtsprozess ausgeführt werden kann.

\subsubsection{Bottom-up-gestützte Serviceidentifikation}

Den bisherigen Ausführungen zur Berichtsserviceidentifikation lag eine Topdown-gestützte Vorgehensweise zugrunde. Um dem für die konzeptionelle Gestaltung eines SOBP geeigneten Meet-in-the-middle-Ansatz gerecht zu werden, ist die bestehende Betrachtung um eine Bottom-up-gestützte Vorgehensweise zu erweitern.

Mit Blick auf das Ziel, Berichtsservices zu verwenden, die sich durch einen hohen Wiederverwendungsgrad auszeichnen, ist im Rahmen der Bottom-upgestützten Serviceidentifikation zum einen zu analysieren, welche geeigneten Berichtsdienste von unternehmungsexternen Providern genutzt werden können. Zum anderen hat die Bottom-up-gestützte Vorgehensweise zum Ziel, alle unternehmungsinternen IS, die von ihnen vorgehaltenen Daten sowie bereitgestellten Funktionen zu analysieren, die sich zur Ausführung der Berichtsprozesse nutzen lassen. Hierbei muss eine Analyse der Systemschnittstellen erfolgen. In diesem Zusammenhang kann es sich z. B. um proprietäre Schnittstellen, Datenbanken und Web Services handeln. Um dabei den Überblick über die betriebenen Schnittstellen zu wahren, sollten alle identifizierten Schnittstellen funktionalen Domänen zugeordnet werden. ${ }^{753}$

Nach der Analyse der Schnittstellen sind in einem nächsten Schritt sämtliche Operationen bzw. Funktionen zu identifizieren, die von den betriebenen IS zur Verfügung gestellt werden. In Frage kommen hierfür alle lesenden und schreibenden Operationen. Ein Beispiel für lesende Operationen ist der automatisierte Import von Bilanzen der Töchter einer Unternehmung. Beispiele für lesende und schreibende Operationen sind die Teilschritte, die im Rahmen eines ETLProzesses ausgeführt werden. ${ }^{754}$ Ferner sind bei der Identifizierung der Operationen auch alle Funktionen zu berücksichtigen, denen eine Verarbeitungslogik zugrunde liegt. Als Beispiel hierfür sind die Berechnungsvorschriften von Kennzahlen zu nennen, die in einer Berichtsanwendung beispielsweise zur Erstellung einer Balanced Scorecard genutzt werden.

Neben der Identifikation von Serviceoperationen spielt auch die Identifikation der Datenobjekte, die von den zugrunde liegenden IS zur Verarbeitung der

753 Vgl. hierzu Offermann (2008), S. 466.

754 Vgl. Abschnitt 5.2.2.2.1 
Geschäfts- und Finanzinformationen verwendet werden, eine wichtige Rolle.755 Als Grundlage zur Identifikation der Datenobjekte lassen sich die semantischen und konzeptionellen bzw. logischen Datenmodelle der IS nutzen. ${ }^{756} \mathrm{Da}$ aufgrund der Vielzahl der zum Einsatz kommenden IS bzw. Datenmodelle die Datenobjekte in diesem Zusammenhang typischerweise unterschiedlich bezeichnet sind, ist es notwendig, die Datenobjekte dieser Datenmodelle zu konsolidieren.

$\mathrm{Da}$ zwischen den Datenobjekten eines Datenmodells vielfältige Beziehungen existieren, reicht es bei der Konsolidierung nicht aus, nur die Bezeichnung der Datenobjekte zu betrachten bzw. anzupassen. Stattdessen sind Verfahren der Schemaintegration sowie des Schema-Mappings anzuwenden, um eine konsistente und korrekte Sicht auf die Bedeutung der Datenobjekte sowie der zwischen den Objekten bestehenden Beziehungen zu erreichen. ${ }^{757}$ Für ähnliche oder gleiche Datenobjekte sind Objekte zu erstellen, die alle Eigenschaften der Datenobjekte enthalten, die sie vereinen. Um die Konsolidierung der Datenmodelle zu erleichtern, ist es dabei empfehlenswert, die Datenobjekte nach funktionalen Domänen zu gruppieren.

\subsubsection{Fachliche Servicegestaltung}

Die fachliche Servicegestaltung ist die letzte Phase der konzeptionellen Gestaltung eines SOBP. Ziel dieser Phase ist es, den Leistungsumfang bzw. die Funktionalität der identifizierten Berichtsservices zu definieren. ${ }^{758}$ Die fachliche Gestaltung von Services hat vor allem vor dem Hintergrund erster SOAUmsetzungen in der Praxis eine große Bedeutung. Diese Projekte haben gezeigt, dass sich die Flexibilitätsanforderungen, die derzeit an Applikationslandschaften gestellt werden, durch die rein technische Zerlegung eines IS in Services nicht zufriedenstellend erfüllen ließen. ${ }^{759}$

Neben einer fachlichen Eignung der Dienste, die durch die Flexibilisierung und/oder Automatisierung der Berichtsprozesse einen betriebswirtschaftlichen Mehrwert generieren sollen, ist bereits bei der fachlichen Servicegestaltung zu

755 Ausgedrückt in der Begriffswelt der Entity-Relationship-Modellierung (ERModellierung) werden im Folgenden als Datenobjekte sowohl die Entity-Types als auch die Attribute der Entity-Types aufgefasst. Zur ER-Modellierung vgl. Gabriel/Röhrs (1995), S. 104f.

$756 \mathrm{Zu}$ den Merkmalen und Unterschieden dieser beiden Ausprägungen eines Datenmodells vgl. Gabriel/Röhrs (1995), S. 104ff.

757 Das Vorgehen zur Schemaintegration sowie zum Schema-Mapping wird bei LESER/ NAUMANN beschrieben. Vgl. Leser/Naumann (2007), S. $116 \mathrm{ff}$.

758 Die Bestimmung des Leistungsumfangs von Services ist von Bedeutung, damit Dienste nicht nur einen technischen, sondern auch einen fachlichen Nutzen generieren. Vgl. hierzu Tilkov/Starke (2007), S. 14.

759 Vgl. Esch et al. (2008), S. 94. 
berücksichtigen, dass sich die identifizierten Services auch für eine spätere Realisierung eignen. ${ }^{760}$ Die Anforderungen, die dies sicherstellen, werden in Abschnitt 5.2.3.1 diskutiert. Die fachliche Berichtsservicegestaltung beginnt in Abschnitt 5.2.3.2 mit der Kategorisierung des Leistungsumfangs der identifizierten Berichtsservices. Darauf aufbauend ist zu überprüfen, inwieweit sich die zur Verfügung stehenden XBRL-Taxonomien zur semantischen Beschreibung der von einem Berichtsservice verarbeiteten Geschäfts- und Finanzinformationen nutzen lassen (Abschnitt 5.2.3.3). In Abschnitt 5.2.3.4 wird ein Vorgehen zur Bewertung der Dienstgüteeigenschaften der im Rahmen der Bottom-upAnalyse identifizierten unternehmungsinternen Services oder Dienste eines unternehmungsexternen Anbieters erarbeitet. Zum Abschluss fokussiert Abschnitt 5.2.3.5 eine Methode zur fachlichen Gestaltung neu zu entwickelnder Berichtsservices.

\subsubsection{Anforderungen zur fachlichen Servicegestaltung}

Mit Blick auf das Ziel einer SOA, eine hohe Wiederverwendbarkeit von Diensten zu ermöglichen, lassen sich fünf Anforderungen zur fachlichen Gestaltung von Services identifizieren. Diese werden nachfolgend erläutert.

\section{Anforderung 1: Normale Serviceoperationen}

Die erste Anforderung fokussiert die Gestaltung so genannter normaler Serviceoperationen. Die Anforderung der Normalität lässt sich aufteilen in die Teilanforderungen der Vollständigkeit und der Redundanzfreiheit. Die Anforderung der Vollständigkeit sieht vor, dass die Operationen, die ein Service ausführt, die gesamte an ihn geforderte Funktionalität abdecken.761 Dies betrifft sowohl schreibende als auch lesende Operationen. Die Redundanzfreiheit zielt darauf $a b$, dass kein Service eingesetzt werden darf, dessen Funktionalität bereits von einem anderen Dienst erbracht wird. ${ }^{762}$ Dies hat folglich zur Konsequenz, dass ein entsprechender Berichtsservice exakt einmal vorkommen darf. Wie HESS/HuMM/Voss in diesem Zusammenhang bemerken, gilt die Forderung der Redundanzfreiheit ausschließlich für Basis- und Funktionsservices. Eine Inkaufnahme redundanter Prozessservices ist hingegen möglich, falls dies aus Gründen der Performance und Nutzungsfreundlichkeit der Schnittstelle erforderlich ist. ${ }^{763}$

$760 \mathrm{Vgl}$. Beverungen/Knackstedt/Müller (2008), S. 223.

761 Vgl. Humm/Juwig (2006), S. 3.

762 Vgl. Gimnich (2007), S. 68.

$763 \mathrm{Vgl}$. Hess/Humm/Voss (2006), S. 403. 


\section{Anforderung 2: Idempotente Serviceoperationen}

Die Anforderung der Idempotenz hat zur Aussage, dass mehrmalige Aufrufe eines Services zu genau dem Ergebnis führen müssen, welches aus der einmaligen Nutzung dieses Dienstes resultiert. ${ }^{764}$ Der wiederholte Aufruf eines Services, der z. B. im Rahmen des Kernprozesses Berichtsproduktion den Kapitalwert einer Investition berechnet, 765 darf folglich nicht zu abweichenden Ergebnissen führen. Gleiche Ergebnisse wiederum setzen voraus, dass sich die Datengrundlage - im hier betrachteten Beispiel die Zahlungsüberschüsse, die Laufzeit der Investition und der zugrunde liegende Diskontierungszinssatz - zwischen den entsprechenden Serviceaufrufen nicht geändert hat.

\section{Anforderung 3: Datenhoheit der Basisservices}

Die Anforderung der Datenhoheit der Basisservices besagt, dass der Zugriff auf die Informationsobjekte eines IS, das Bestandteil einer SOA ist, über Basisservices erfolgen muss. D. h., alle lesenden und schreibenden Serviceoperationen sind mit Hilfe dieser Serviceart durchzuführen. Ziel ist es dabei, dass jeder Basisservice einen disjunkten Ausschnitt aller Informationsobjekte verwaltet.

\section{Anforderung 4: Wahrung der Abhängigkeitsbeziehungen zwischen den Servicekategorien}

In Abschnitt 4.2.2.1 ist bereits deutlich geworden, dass sich die im Rahmen einer SOA zur Anwendung kommenden Services anhand der Kategorien Bestandsservices, Funktionsservices und Prozessservices systematisieren lassen. Um Services miteinander verknüpfen zu können, ist es erforderlich, die Abhängigkeitsbeziehungen zwischen diesen drei Servicekategorien aufrechtzuerhalten. Die Abhängigkeitsbeziehungen zwischen zwei Services können hierbei als „,kennt"-, ,ruft auf"- oder ,bezieht Daten von“-Aussagen formuliert werden. In Anlehnung an HESS/HUM/VOß lassen sich dann folgenden Abhängigkeitsbeziehungen identifizieren: ${ }^{766}$

- Prozessservices sollen höchstens abhängig untereinander sowie abhängig zu Funktions- und Bestandsservices sein.

- Funktionsservices sollen höchstens abhängig untereinander sein sowie abhängig zu Bestandsservices sein.

- Bestandsservices sollen ausschließlich abhängig untereinander sein.

764 Wie HUMM/JUWIG bemerken, stammt diese Idee aus der Batch-Verarbeitung. Die Idempotenz garantiert in diesem Kontext, dass ein versehentlich angestoßener Batch-Lauf nicht zu einem unerwünschten bzw. fehlerhaften Ergebnis führt. Vgl. Humm/Juwig (2006), S. 3.

765 Zur Kapitalwertmethode vgl. z. B. Busse von Colbe/Laßmann (1990), S. 6ff; Ross/ Westerfield/Jaffe (1999), S. 64ff.

766 Vgl. Hess/Humm/Voss (2006), S. 401. 


\section{Anforderung 5: Kontextfreie Serviceoperationen}

Eine weitere Anforderung betrifft die Kontextfreiheit der Serviceoperationen. Nach Hess/Hum/Voss lässt sich diese Anforderung aus der Perspektive des Session- sowie des Transaktionskontextes betrachten. ${ }^{767}$ Serviceoperationen, die sessionkontextfrei sind, zeichnen sich dadurch aus, dass sie keine Informationen über den Servicenutzer, über die zugrunde liegenden Sessions sowie den Aufrufkontext benötigen. Ebenso soll die Berechtigungsprüfung außerhalb der fachlichen Funktionalität bzw. Operation des jeweiligen Services durchgeführt werden.

Die Anforderung, kontextfreie Serviceoperationen zu gestalten, gewährleistet darüber hinaus, dass sich Services als Transaktionen auffassen lassen. D. h., ein Dienst wird entweder vollständig oder gar nicht ausgeführt. Die Kontextfreiheit von Serviceoperationen hat damit zur Folge, dass kompensierende Operationen ebenfalls implementiert werden müssen, die beispielsweise im Falle einer fehlerhaften Ausführung eines Service aufgerufen werden. ${ }^{768}$

\subsubsection{Kategorisierung der potenziellen Berichtsservices}

Bevor Dienste implementiert werden, ist beim Entwurf der Dienste die Aufgabe zu erfüllen, Services in verschiedene Kategorien einzuordnen. ${ }^{769}$ Allgemein wird mit der Kategorisierung der Services das Ziel verfolgt, die betriebswirtschaftliche Funktionalität der in einer SOA zum Einsatz kommenden Dienste klar voneinander abzugrenzen. ${ }^{770}$ Die Anforderung, die identifizierten Services hinsichtlich ihres Aufgabenumfangs zu klassifizieren, ist daher auch bei der fachlichen Gestaltung der Berichtsservices von großer Bedeutung.

Die Kategorisierung der Berichtsservices fokussiert nicht nur auf die (betriebswirtschaftlichen) Aufgaben, die ein Berichtsservice erbringen soll. Auch unterstützende Aktionen, die (als Berichtsservice gekapselt) zur Erfüllung einer betriebswirtschaftlichen Funktionalität beitragen, sind anhand geeigneter Kategorien zu klassifizieren. Ein Beispiel für einen Berichtsservice, der derartige Aktionen ausführt, ist ein Dienst, der das Einlesen von Geschäfts- und Finanzinformationen ermöglicht, die im Rahmen des Kernprozesses Berichtsproduktion verwendet werden. Als geeignete Klassen lassen sich für die Kategorisierung der potenziellen Berichtsservices die in Abschnitt 4.2.2.1 vorgestellten Servicearten Datenservices, Funktionsservices und Prozessservices verwenden.

Mit der Kategorisierung von Berichtsservices lassen sich zwei Teilziele erfüllen. Erstens bewirkt die Zuweisung eines Services zu einer Serviceart eine bes-

Vgl. Hess/Humm/Voss (2006), S. 404.

768 Vgl. Humm/Juwig (2006), S. 3.

$769 \mathrm{Vgl}$. Beverungen/Knackstedt/Müller (2008), S. 223.

770 Vgl. Klose/Knackstedt (2007), S. 47. 
sere Pflege des Serviceportfolios. Da zu erwarten ist, dass der Servicebestand im Laufe der Nutzung einer SOA wachsen wird, ist frühzeitig Sorge zu tragen, dass die Services anhand eines zusätzlichen Kriteriums „Serviceart" systematisiert werden und sich folglich im Laufe ihrer Nutzung in einem Serviceverzeichnis leichter auffinden lassen. Zweitens ist die Kategorisierung der Services erforderlich, um Anforderung 4 bei der fachlichen Gestaltung von Services zu erfüllen. ${ }^{771}$ Diese Anforderung beinhaltet, dass Abhängigkeiten zwischen den Servicearten gewahrt werden müssen, um eine effektive (d. h. eine konsistente und korrekte) Gestaltung der Services sowie ihrer Beziehungen zueinander mit dem Ziel zu gewährleisten, eine effiziente Ausführung der Serviceorientierten Prozesse zu ermöglichen.

\subsubsection{Zuweisung von XBRL-Taxonomieelementen}

Nachdem die in Frage kommenden Berichtsservices einer Kategorie zugeordnet wurden, ist zu entscheiden, welche XBRL-Taxonomien zum Einsatz kommen sollen, um die zu verarbeitenden Geschäfts- und Finanzinformationen semantisch zu beschreiben. Je nach verfolgtem Berichtszweck können XBRL FRTaxonomien, die XBRL GL-Taxonomie sowie beide Taxonomiekategorien in kombinierter Form zum Einsatz kommen. $\mathrm{Zu}$ prüfen ist, welche Inputinformationen, die als Eingabewerte zur Ausführung einer Serviceoperation benötigt werden, sich mit Hilfe der zur Verfügung stehenden XBRL-Elemente der jeweiligen Taxonomie auszeichnen lassen. In gleicher Weise ist mit den Outputwerten zu verfahren.

\subsubsection{Bewertung der Dienstgüte bereits vorhandener interner oder ex- terner Berichtsservices}

Bevor mit der fachlichen Gestaltung neuer Berichtsservices begonnen wird, ist zu prüfen, inwieweit sich die im Rahmen der Bottom-up-Analyse identifizierten unternehmungsinternen Services oder Dienste eines unternehmungsexternen Anbieters zur konzeptionellen Gestaltung des SOBP nutzen lassen. Um die Eignung eines Berichtsservices zu bestimmen, ist es nötig, die Dienstgüte der potenziellen Services einer kritischen Bewertung zu unterziehen. Die Bewertung der Dienstgüte der Services ist von Bedeutung, da die am höchsten bewerteten Dienste potenzielle Kandidaten für eine softwaretechnische Implementierung darstellen. ${ }^{772}$

Da bei einem SOBP alle Vorgänge zur Verarbeitung der berichteten Geschäfts- und Finanzinformationen durch die Inanspruchnahme von Services

Vgl. Abschnitt 5.2.3.1.

772 Vgl. hierzu Gimnich (2007), S. 73. 
durchgeführt werden sollen, 773 muss bei der Gestaltung und dem Betrieb eines derartigen Prozesses gewährleistet sein, dass nur diejenigen Dienste zum Einsatz kommen, welche die im Rahmen des Servicevertrags vereinbarten Dienstgüteeigenschaften erfüllen. ${ }^{774}$ Die Einhaltung der Dienstgüteeigenschaften ist sowohl während der Nutzung als auch Entwicklung neuer Services oder Modifizierung bestehender Dienste zu gewährleisten. In gleicher Weise müssen Services, die je nach Berichtszweck von unternehmungsexternen Akteuren bereitgestellt werden, hinsichtlich ihrer Eignung überprüft werden.

Um die Dienstgüteeigenschaften zu überwachen, reicht es daher nicht aus, geeignete Qualitätskriterien zu definieren und technische Komponenten einzusetzen, die die Einhaltung der Dienstgüteeigenschaften garantieren. Darüber hinaus ist es erforderlich, Bewertungsmechanismen einzubinden, welche Entscheidungen untermauern, ob ein neu entwickelter, ein modifizierter oder extern bereitgestellter Service in das Dienstportfolio der Unternehmung aufgenommen und zur Nutzung freigegeben werden kann.

Zur Systematisierung der Inhalte einer Servicebeschreibung lassen sich die Informationen, die anhand quantitativer Kriterien beurteilt werden können, von denen unterscheiden, die eine qualitative Bewertung erfordern. ${ }^{775} \mathrm{Im}$ Vergleich zu den qualitativen Kriterien bietet der Einsatz von quantitativen Kriterien den Vorteil, dass ihnen objektiv messbare Werte zugeordnet werden. Sowohl die quantitativen als auch die qualitativen Dienstgüteeigenschaften sind Gegenstand von Service Level Agreements (SLA). ${ }^{776}$ Ein SLA gleicht einem Vertrag zwischen einem Servicegeber und einem Servicenehmer und beinhaltet Parameter, die als Service Level Objects (SLO) bezeichnet werden und das Qualitätsniveau der zu erbringenden Funktionalität spezifizieren. ${ }^{777}$ Die Dienstgüte wird in diesem Zusammenhang auch als Quality of Service (QoS) bezeichnet. ${ }^{778}$

773

Vgl. Abschnitt 4.1.

Generell stellen Verträge die Voraussetzung dar, dass Kooperationen zustande kommen. Ein Vertrag enthält alle expliziten oder impliziten Vereinbarungen zwischen den beteiligten Vertragsparteien, die eine bindende Wirkung für diese haben. Vgl. Scholtis (1998), S. 23. Mit Blick auf den vorliegenden Untersuchungsgegenstand wird ein derartiger Vertrag im Rahmen eines Service Level Agreements abgeschlossen.

Diese Unterscheidung geht auf den Vorschlag von BERBNER ET AL. zurück. Vgl. Berbner et al. (2005), S. 270f. Einen alternativen Ansatz zur Systematisierung der Informationen, die in einer Servicespezifikation enthalten sind, präsentiert LIEBHART. Seinem Vorschlag zufolge enthält eine Servicebeschreibung organisatorische, technische sowie planerische Informationen zu einem Service. Vgl. Liebhart (2007), S. 164ff.

Vgl. Josuttis (2007), S. 386.

Vgl. Hermann/Müller (2008), S. 237; Herden et al. (2006), S. 135; Berbner et al. (2005), S. 270.

Vgl. Ernst (2000), S. 675. 
Nachdem in Abschnitt 5.2.3.4.1 die Qualitätsparameter erläutert werden, die sich zur Beurteilung von Services nutzen lassen, stellt Abschnitt 5.2.3.4.2 das Vorgehen zur Bewertung der Servicequalität vor.

\subsection{Qualitätskriterien zur Bewertung der Berichtsservices}

Unter den objektiv messbaren Anforderungen lassen sich als mögliche Kriterien die Verfügbarkeit (Availability), Leistungsfähigkeit (Performance), Fehlerhäufigkeit (Error Rate) sowie die Kosten der Servicenutzung anführen. ${ }^{779}$ Eine Übersicht über diese und weitere quantitative und qualitative Kriterien zeigt Abbildung 43.

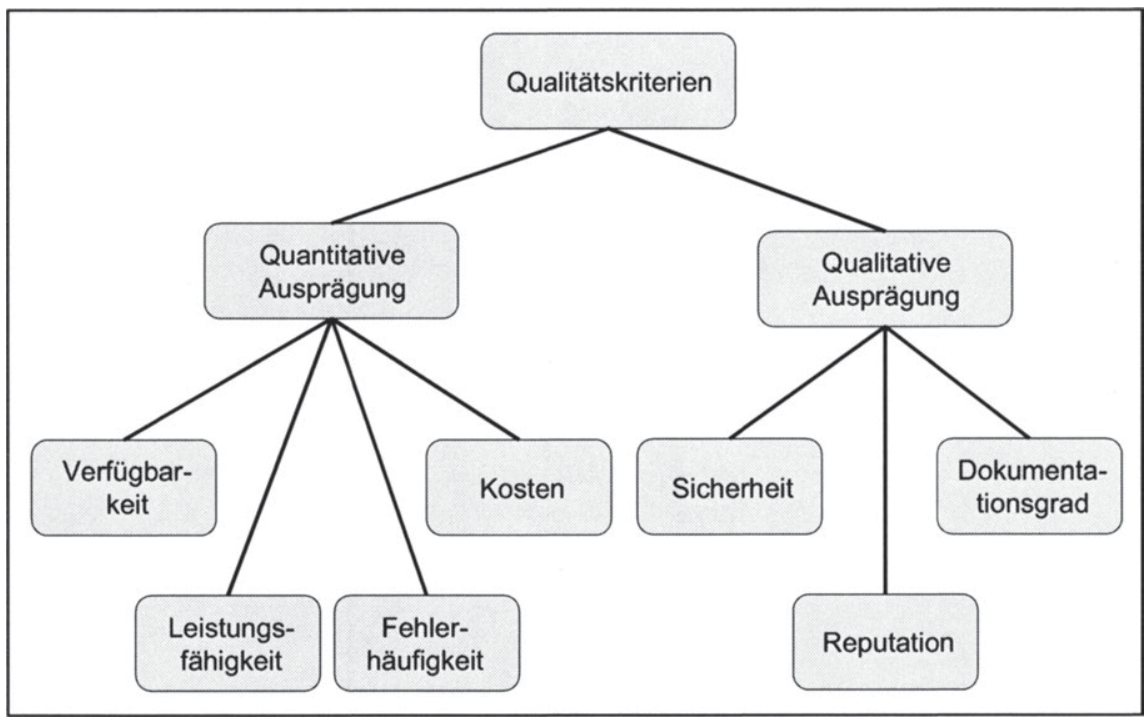

Abbildung 43: Übersicht der Qualitătskriterien zur Beurteilung der Dienstgüte

In Anlehnung an den Begriff der Verfügbarkeit eines IS informiert die Verfügbarkeit eines Services über die Wahrscheinlichkeit, dass ein Dienst zur Nutzung zur Verfügung steht. ${ }^{780}$ Ein Service lässt sich als verfügbar bezeichnen, wenn es diesem gelingt, eine Anfrage in einer geforderten Zeit zu verarbeiten. Das Kriterium der Leistungsfähigkeit wird in die Teilkriterien Durchsatz (Throughput) und Antwortzeit (Response Time) separiert. ${ }^{781}$ Die Antwortzeit setzt sich wie-

Vgl. Berbner et al. (2005), S. 270.

Vgl. Hansen/Neumann (2005), S. 287.

781 Vgl. Berbner et al. (2005), S. 270. 
derum aus der Zeit zusammen, die zur Übertragung und Bearbeitung der Anfrage verwendet wird, sowie der Zeit, die ein Service benötigt, um die angeforderte Antwort dem Servicenutzer zur Verfügung zu stellen. Die Fehlerhäufigkeit liefert eine Aussage über die Anzahl der Fehler, die innerhalb eines bestimmten Zeitraums aufgetreten sind. Als Fehlerursachen kommen z. B. fehlerhafte Ergebnisse oder Übertragungsfehler in Frage.

Neben der Verfügbarkeit, Leistungsfähigkeit und Fehlerhäufigkeit beschreiben die Kosten, die mit der Servicenutzung verbunden sind, eine weitere wichtige Eigenschaft eines Berichtsservice. ${ }^{782}$ Zur Abrechnung eines Services steht eine Vielzahl von Varianten zur Verfügung. Diesen liegt jeweils eine nutzenabhängige oder nutzenunabhängige Abrechnung zugrunde. Während bei der nutzenunabhängigen Abrechnung die Servicenutzung zu einem zuvor vereinbarten Pauschaltarif erfolgt, können Services im Rahmen einer nutzenabhängigen Abrechnung beispielsweise nach der Anzahl der Aufrufe in Rechnung gestellt werden. Darüber hinaus bietet sich hier die Möglichkeit an, Dienste nach dem Datenvolumen oder der in Anspruch genommenen Nutzungsdauer abzurechnen.

Bei den qualitativen Kriterien sind vor allem die Sicherheit, die Reputation des Serviceproviders sowie der Dokumentationsgrad eines Services relevant. ${ }^{783}$ Die Anforderung der Sicherheit lässt sich anhand der Teilkriterien Authentizität, Autorisierung, Vertraulichkeit und Datenverschlüsselung betrachten. Die Reputation des Serviceproviders liefert eine Aussage zu den Erfahrungen, die zum gegebenen Zeitpunkt mit den Diensten eines Serviceanbieters gemacht wurden. Dieses Kriterium spielt insbesondere bei der Nutzung von Services eine Rolle, die von einem unternehmungsexternen Anbieter bereitgestellt werden. Hierbei ist zu evaluieren, ob es sich bei dem Serviceprovider um einen seriösen und bekannten Anbieter handelt. Ein weiterer Aspekt, der in diesem Zusammenhang die Bewertung der Reputation eines Serviceproviders beeinflusst, richtet sich auf die Existenz sowie die Qualität einer Supportabteilung des Dienstanbieters. Insbesondere im Fall des Serviceausfalls oder bei notwendig gewordenem Änderungsbedarf eines Dienstes ist dieses Kriterium von tragender Bedeutung.

Der Dokumentationsgrad eines Services repräsentiert, ob und inwieweit Änderungen, welche im Laufe des Lebenszyklus eines Dienstes anfallen, nachvollziehbar belegt sind. Informationen, die den Dokumentationsgrad eines Berichtsservices beschreiben, müssen beispielsweise darüber Aufschluss geben, wer den Berichtsservice erstellt hat, wann der Service das letzte Mal geändert wurde und warum er modifiziert wurde. Auf diese Weise lassen sich Verantwortlichkeiten für die Pflege eines Services zuweisen. Das Kriterium des Dokumentationsgrads

782 Auf die Bedeutung, zur Dynamisierung von Serviceketten Bepreisungs- und Abrechnungsmodelle einzusetzen, verweisen Beimborn/Mintert/Weitzel (2002), S. 278. 
ist vor allem für Berichtsservices von Bedeutung, die häufig angepasst oder verändert werden müssen.

\subsection{Vorgehen zur Bewertung der Dienstgüte}

Bei der Vorgehensweise zur Bewertung der Dienstgüte der identifizierten unternehmungsinternen und -externen Berichtsservices ist $\mathrm{zu}$ unterscheiden, ob sich qualitative und/oder quantitative Kriterien verwenden lassen. Um eine Bewertung der quantitativ messbaren Kriterien (Verfügbarkeit, Leistungsfähigkeit, Fehlerhäufigkeit und Kosten) vorzunehmen, sind adäquate Gewichte festzulegen, welche die Bedeutung der jeweiligen Teilanforderung für die Beurteilung der Dienstgüte widerspiegeln. ${ }^{784} \mathrm{Da}$ die Ausprägungen, die zur Bewertung der quantitativen Kriterien zum Einsatz kommen, in verschiedenen Einheiten vorliegen, 785 ist es zweckmäßig, die zugeordneten Werte zu normalisieren. ${ }^{786}$ Auf diese Weise lässt sich die Vergleichbarkeit der eingesetzten Services realisieren. Dabei hängt die Normalisierung davon ab, ob das Ziel des jeweiligen Kriteriums ein hoher oder niedriger Wert ist. Während bei der Antwortzeit und der Fehlerhäufigkeit ein kleiner Wert angestrebt wird, liegt bei der Verfügbarkeit und beim Durchsatz der umgekehrte Fall vor. Die Normalisierung der Antwortzeit und der Fehlerhäufigkeit lässt sich durchführen, indem aus einer Auswahl $n$ der zur Verfügung stehenden Services der Dienst mit der längsten Antwortzeit bzw. der größten Fehlerhäufigkeit durch den zu bewertenden Service dividiert wird.

Bei Kriterien, bei denen möglichst hohe Werte wünschenswert sind (Verfügbarkeit und Durchsatz), werden der Zähler und der Nenner vertauscht. D. h., der zu bewertende Service wird durch den Dienst dividiert, der die geringste Wertausprägung bezüglich des betrachteten Kriteriums (Verfügbarkeit und Durchsatz) aufweist.

Im Vergleich zu den quantitativen Kriterien ist bei den qualitativen Kriterien eine manuelle Bewertung der Dienstgüteeigenschaft seitens der ITVerantwortlichen und/oder des Berichtsempfängers erforderlich. Zur qualitativen Beurteilung des Kriteriums lässt sich eine Bewertungsmatrix einsetzen, wie

784 Die Gewichtung ist eine häufig angewendete Möglichkeit zur Lösung von Zielkonflikten bei multikriteriellen Entscheidungsproblemen. Die verschiedenen quantitativ und qualitativ bewerteten Kriterien werden jeweils mit Faktoren gewichtet. Diese individuellen Gewichtungsfaktoren sind Maßstabsfaktoren, die das Verhältnis der einzelnen Faktoren zum Gesamtnutzen fixieren. Formal besteht die Zielgewichtung in der Multiplikation der Wertausprägungen mit ihren nichtnegativen Gewichten und der Addition der so gewichteten Kriterien. Auf diese Weise lässt sich ein Service bestimmen, der den höchsten Nutzen liefert. Vgl. Jenner (2000), S. 330.

Siehe hierzu Abbildung 44.

786 Vgl. Berbner et al. (2005), S. 271. 
sie exemplarisch in Abbildung 44 dargestellt ist. Festzuhalten ist, dass der $\mathrm{zu}$ bewertende Service in diesem Beispiel nutzungsabhängige Kosten von 10 Cent pro Aufruf verursacht. Zur Authentifizierung kommt X.509 zum Einsatz,787 während die Autorisierung auf Basis des Advanced Encryption Standard (AES) erfolgt. ${ }^{788}$ Die bisherigen Erfahrungen mit dem Provider des Services sowie mit dem Service selbst wurden als gut bzw. sehr gut eingeschätzt. Externe Referenzen sind existent und lassen sich über ein Informationsportal einsehen.

Die letzte Spalte verdeutlicht, dass die qualitativen Kriterien individuell bewertet werden müssen, wobei im vorliegenden Beispiel eine Bewertung von 5 Punkten auf eine herausragende Eignung des Dienstes im Hinblick auf die zugrunde liegende Anforderung deutet. Neben den individuellen Bewertungen enthält die Bewertungsmatrix die Gewichtungsfaktoren, die sowohl für die quantitativen als auch für die qualitativen Faktoren festgelegt werden müssen.

\begin{tabular}{|c|c|c|c|}
\hline 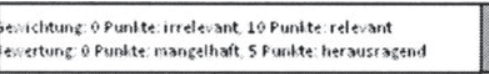 & Beurteilung & $\begin{array}{c}\text { Gewichtungsfaktor } \\
\text { (0-10 Punkte) }\end{array}$ & $\begin{array}{l}\text { Bewertuns } \\
\text { (0-5 Punkte) }\end{array}$ \\
\hline \multicolumn{4}{|l|}{ Technische Eigenschaften } \\
\hline Verfïgbarkeit (in \%) & 99 & 8 & - \\
\hline Durchsatz (in n Nachrichten pro Minute) & 360 & 7 & - \\
\hline Antwortzeit (in Millisekunden) & 33 & 9 & - \\
\hline Fehlerhäufigkeit (n fehler pro Tag) & 1 & 10 & - \\
\hline \multicolumn{4}{|l|}{ Kosten } \\
\hline Tarif (in Cent/Aufruf) & 10 & 6 & - \\
\hline \multicolumn{4}{|l|}{ Sicherheit } \\
\hline Authentifizierung & $\times .509$ & 8 & 5 \\
\hline Autorisierung & AES & 9 & 4 \\
\hline \multicolumn{4}{|l|}{ Reputation } \\
\hline Bisherige Erfahrungen mit dem Provider & Gute Erfahrungen & 5 & 4 \\
\hline $\begin{array}{r}\text { Bisherige Erfahrungen mit dem Web } \\
\text { Service }\end{array}$ & Sehr gute Erfahrungen & 5 & 5 \\
\hline Externe Referenzen & Informationsportal & 6 & 5 \\
\hline Dokumentationsgrad & Gut dokumentiert & 7 & 4 \\
\hline
\end{tabular}

\section{Abbildung 44: Beispiel einer Bewertungsmatrix}

Um eine abschließende Bewertung der Dienstgüte vorzunehmen, ist es erforderlich, die gewichteten qualitativen und die gewichteten sowie normalisierten quantitativen Kriterien zu einem Gesamtergebnis zu verdichten. Für diesen

787 Bei X.509 handelt es sich um einen Standard für digitale Zertifikate. Vgl. Schwenk (2005), S. 22ff.; Federrath/Pfitzmann (2006), S. 283.

788 AES ist ein symmetrisches Verschlüsselungsverfahren, das mit einer Schlüssellänge von 128, 192 und 256 Bit arbeitet. Vgl. Schwenk (2005), S. 8 f. 
Zweck lässt sich z. B. eine lineare Bewertungsfunktion nutzen, ${ }^{789}$ so dass sich die Dienstgüte eines Services als Summe der gewichteten Wertausprägungen der Einzelkriterien ermitteln lässt. Auf diese Weise können sowohl die unternehmungsexternen als auch die -internen Services vor dem Hintergrund ihrer Dienstgüteeigenschaften miteinander verglichen werden. Durch die Wahl der Gewichte lassen sich zudem einsatzspezifische Besonderheiten berücksichtigen. ${ }^{790}$

\subsubsection{Fachliche Gestaltung neuer Berichtsservices}

Charakteristisch für das Berichtswesen ist, dass zur Erstellung der berichteten Geschäfts- und Finanzinformationen Berechnungsvorschriften genutzt werden (müssen), die entweder vom Gesetzgeber im Rahmen eines Rechnungslegungsstandards vorgegeben sind oder sich als geeignet zur Erfüllung der Berichtszwecke erwiesen haben, ${ }^{791}$ jedoch keiner gesetzlichen Ermittlungsgrundlage unterliegen. Während die Ermittlung eines im Geschäftsbericht zu veröffentlichenden Gewinns oder Verlustes vom Gesetzgeber durch das HGB vorgegeben ist, liegt eine derartige Vorgabe beispielsweise für die Berechnung des Auftragsbestands nicht vor. Vielmehr ergibt sich das Verständnis, um was es sich bei der Kennzahl „Auftragsbestand“ handelt, aus der Definition dieser Größe durch die Fachabteilung, die diese Kennzahl nutzt. Um die Aktionen und damit die fachliche Funktionalität eines Berichtsservices zu spezifizieren ist - sofern vorhanden - auf bereits existierende Vorgehensweisen bzw. Berechnungsvorschriften zurückzugreifen, die dokumentieren, wie sich Kennzahlen ermitteln lassen bzw. zu ermitteln sind.

Dieser Abschnitt führt auf, welche Schritte notwendig sind, um Berichtsservices, die infolge der Top-down-Analyse als Servicekandidaten identifiziert wurden, unter Einbezug einer dokumentierten Vorgehensweise fachlich gestaltet werden können. Zur fachlichen Servicegestaltung sind fünf sequenziell auszuführende Schritte durchzuführen, die im Folgenden vorgestellt werden.792

\section{Schritt 1: Identifikation möglicher Vorgehensweisen}

$\mathrm{Zu}$ Beginn der fachlichen Servicegestaltung ist die Ausgangssituation zu ermitteln, was Aufschluss darüber geben soll, ob für eine Aktivität bereits eine Vor-

789 Mit Hilfe einer Bewertungsfunktion, die auch als Entscheidungsregel oder Präferenzfunktional bezeichnet wird, lassen sich die Präferenzen eines Entscheidungsträgers abbilden. Bewertungsfunktionen werden zur Unterstützung von Entscheidungen eingesetzt. Vgl. Werners (2008), S. 25.

Vgl. Berbner et al. (2005), S. 271.

791 Vgl. Abschnitt 2.1.3.

792 Vgl. Winkler (2007), S. $261 \mathrm{ff}$. 
gehensweise dokumentiert ist. Die Identifikation möglicher Vorgehensweisen sowie alle weiteren in diesem Abschnitt vorgestellten Schritte sind für jede aus dem Berichtsprozessmodell heraus identifizierte Aktivität durchzuführen. In diesem Zusammenhang lassen sich drei Fälle unterscheiden.

Liegt keine dokumentierte Vorgehensweise vor (Fall 1), muss eine entsprechende Vorgehensweise für die fachliche Funktionalität der betrachteten Aktion erstellt werden (Zustand 1.1). ${ }^{793}$ Eine derartige Konstellation liegt etwa dann vor, wenn eine Kennzahl berichtet werden soll, die weder eine gesetzliche Grundlage hat noch jemals in der Unternehmung für die Zwecke der Unternehmungssteuerung erhoben wurde. In diesem Fall ist eine Vorgehensweise zu erarbeiten und für die fachliche Servicegestaltung zu nutzen.

Kann auf eine dokumentierte Vorgehensweise zurückgegriffen werden (Fall 2), lässt sich diese zur fachlichen Gestaltung der Aktion übernehmen (Zustand 1.2). Dieser Fall ist z. B. dann gegeben, wenn Jahresabschlussinformationen erstellt werden müssen. Ein anderes Beispiel einer dokumentierten Vorgehensweise ist die Ermittlung des ROI als Spitzenkennzahl eines Kennzahlenbaums. ${ }^{794}$

Letztlich kann ein Zustand gegeben sein (Fall 3), bei dem mehrere Vorgehensweisen zur Verfügung stehen (Zustand 1.3). In diesem Fall ist in einem nächsten Schritt zu entscheiden, welche Vorgehensweise im Hinblick auf das jeweilige Anwendungsszenario der Aktion geeignet erscheint. Eine derartige Konstellation liegt beispielsweise dann vor, wenn eine zu berichtende Kennzahl (wie der Auftragsbestand) von verschiedenen Fachbereichen ohne explizite Erläuterung unterschiedlich gedeutet wird. ${ }^{795}$ Eine unterschiedliche Deutung wirkt sich wiederum auf die Berechnung der Kennzahl aus, ebenso wie auf die Interpretation der Ergebnisse. Für den Vertrieb ist es z. B. wichtig zu wissen, wie viele Aufträge insgesamt getätigt wurden (Kundenauftragsbestand), während die Produktion ihre Planung darauf ausrichtet, wie viele Aufträge sich gerade an den Maschinen befinden oder noch anstehen (Produktionsauftragsbestand).

\section{Schritt 2: Bestimmung sich ausschließender Voraussetzungen}

Von den oben genannten drei Ausgangssituationen bzw. Fällen ist die zuletzt genannte Konstellation (Zustand 1.3) für das weitere Vorgehen von übergeordneter Bedeutung, da sich aus dieser weitere Schritte für die fachliche Gestaltung der Berichtsservices ergeben. Der weitere Verlauf zur fachlichen Servicegestaltung wird dabei von zwei wesentlichen Zuständen beeinflusst.

793 Im Folgenden steht die zweite Zahl nach dem Punkt für die Ausprägung des jeweiligen Zustands, während die Zahl vor den Punkt informiert, bei welchem Schritt zur fachlichen Servicegestaltung der jeweilige Zustand auftritt.

795 Vgl. Lehmann/Ellerau (1997), S. 84. 
Der erste Zustand ist dadurch charakterisiert, dass die zur Verfügung stehenden Vorgehensweisen verschiedene Voraussetzungen beinhalten, die sich gegenseitig ausschließen (Zustand 1.3.1). In diesem Fall ist keine Auswahl der Vorgehensweisen erforderlich, da für die jeweils vorliegenden Voraussetzungen immer nur eine Vorgehensweise zur Durchführung geeignet ist.

Bei der zweiten Alternative schließen sich die vorliegenden Voraussetzungen zur Anwendung der Vorgehensweisen nicht aus (Zustand 1.3.2). Dies hat zur Konsequenz, dass sich alle zur Verfügung stehenden Vorgehensweisen potenziell in Form eines Services in einer Unternehmung realisieren lassen. Da im Gegensatz zum ersten Fall bei der zweiten Variante (Zustand 1.3.2) mehrere Vorgehensweisen für den zugrunde liegenden Anwendungsfall in Frage kommen, erfordert nur der zweite Fall eine detaillierte Betrachtung.

\section{Schritt 3: Bestimmung einer effizienten Vorgehensweise}

$\mathrm{Zu}$ untersuchen ist im Folgenden, welche Vorgehensweise für den gegebenen Anwendungsfall am besten geeignet ist. Zur Unterstützung einer derartigen Betrachtung lässt sich das Effizienzkriterium verwenden. ${ }^{796}$ Eine Vorgehensweise ist dabei effizient, wenn sie im Vergleich zu einer alternativen Vorgehensweise nicht schlechter abschneidet. Auf diese Weise lässt sich ein Ausschluss ineffizienter Vorgehensweisen durchführen. Um die Eignung der jeweiligen Vorgehensweise zu bewerten, müssen geeignete Evaluationskriterien verwendet werden. Die zur Anwendung kommenden Kriterien müssen dabei insbesondere die fachlichen Eigenschaften der Berichtsservices berücksichtigen. Hierfür lassen sich folgende Kriterien identifizieren:

\section{Grad der Genauigkeit}

Der Grad der Genauigkeit eines zu ermittelnden Werts hat unmittelbaren Einfluss auf die Dauer der Ausführung einer Aktion. Lautet die Forderung beispielsweise, eine Kennzahl mit mehreren Nachkommastellen zu berechnen, sind höhere Antwortzeiten beim Aufruf des betreffenden Dienstes zu erwarten.

\section{Grad der Korrektheit}

Bei der Bedingung „Grad der Korrektheit“ geht es um die Wahrscheinlichkeit, dass das von einem Berichtsservice ermittelte Ergebnis widerspruchsfrei ist und den tatsächlichen Wahrheitsgehalt widerspiegelt. Sind hohe Ansprüche an die Korrektheit eines Ergebnisses gegeben, ist zu überprüfen, ob und inwieweit 
Korrektheits- bzw. Plausibilitätsprüfungen in den betreffenden Dienst eingebunden werden müssen. ${ }^{797}$

\section{Grad der Objektivität}

Diese Bedingung fokussiert die semantische Dimension des von einem Berichtsservice berechneten Ergebnisses. Ein objektives Ergebnis liegt vor, wenn es sich eindeutig interpretieren lässt. Folglich ist in diesem Zusammenhang zu prüfen, ob bei der Verarbeitung der Geschäfts- und Finanzinformationen in einer Aktion, eine XBRL-Taxonomie vorgeschaltet werden muss, die gewährleistet, dass nur diejenigen Informationen, denen im Vorfeld XBRL-Elemente zugewiesen wurden, sowohl als Input- als auch als Outputwerte Gegenstand einer dokumentierten Vorgehensweise sein können.

\section{Grad der Aktualität}

Der „Grad der Aktualität“ stellt ebenfalls eine wichtige Bedingung zur Auswahl einer geeigneten Vorgehensweise dar. Durch diese Bedingung wird ermittelt, wie hoch der Aktualitätsgrad der Inputinformationen sein muss, die für die Ausführung der betrachteten Aktion als Eingabeparameter notwendig sind.

Nach Anwendung des Effizienzkriteriums können sich für den Zustand 1.3.2 zwei Folgezustände ergeben. Zum einen lässt sich eine dominante Vorgehensweise ermitteln, die im Vergleich zu den Alternativen mindestens in einem Bewertungskriterium besser abschneidet, während sie hinsichtlich der anderen Bewertungskriterien mindestens als gleichwertig eingestuft werden kann ( $\mathrm{Zu}-$ stand 1.3.2.1). Zum anderen ist der Fall denkbar, dass sich nach Anwendung des Effizienzkriteriums keine dominante Vorgehensweise ermitteln lässt (Zustand 1.3.2.2). Abbildung 45 fasst die Schritte 1 bis 3 zusammen.

797 Derartige Plausibilitätsprüfungen spielen insbesondere bei der Durchführung von ETLProzessen eine große Rolle. Hierzu werden entsprechende Plausibilitätsroutinen verwendet, um möglichst frühzeitig Fehler bei der Aufbereitung der Daten festzustellen, die ansonsten erst zum Ende eines ETL-Prozesses, der je nach DWH-Anwendung über mehrere Minuten bis zu Stunden dauern kann, auffallen würden. 


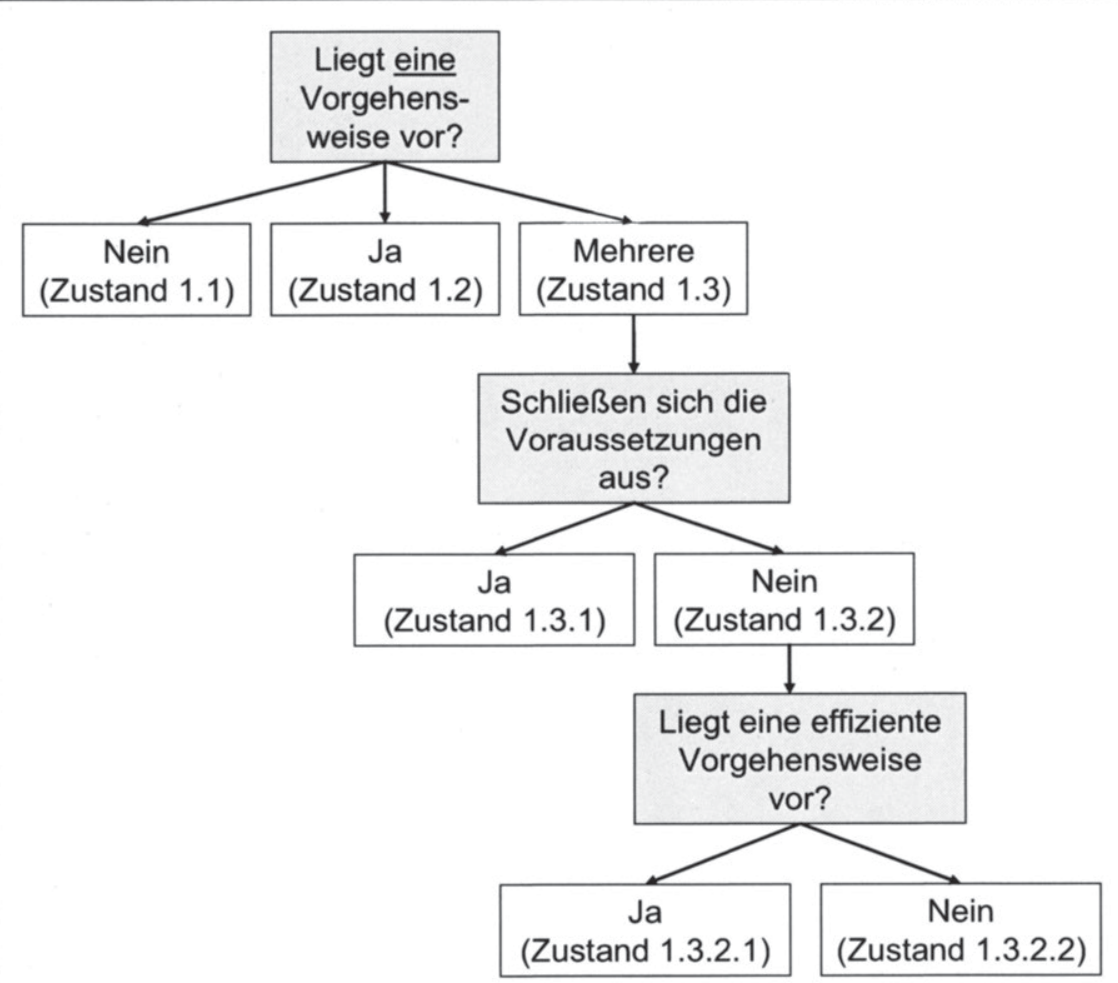

Abbildung 45: Übersicht der Schritte 1 bis 3 bei der fachlichen Servicegestaltung

\section{Schritt 4: Einbindung zusätzlicher Auswahllogiken}

Um im Rahmen der angestrebten Servicenutzung bei mehreren nicht dominierten Vorgehensweisen eine eindeutige Auswahl der Vorgehensweise zu ermöglichen, sind zusätzliche Bedingungen in den Prozess zur Auswahl der jeweiligen Aktion zu integrieren. Derartige Bedingungen bzw. Auswahllogiken bedürfen geeigneter Kriterien, anhand derer sich die Selektion der Aktionen beurteilen lässt.

Eine eindeutige Auswahl einer Vorgehensweise lässt sich gewährleisten, wenn die möglichen Vorgehensweisen in eine Ordnung gebracht werden. $\mathrm{Zu}$ diesem Zweck lassen sich die in Schritt 3 erarbeiteten fachlichen Kriterien heranziehen. Zusätzlich ist eine Priorisierung der angeführten Bewertungskriterien vorzunehmen, um zu gewährleisten, dass bei der Ausführung eines Services diejenige Vorgehensweise zur Anwendung kommt, die das am höchsten priorisierte fachliche Evaluationskriterium am besten erfüllt. Dieses Kriterium kann je 
nach Einsatzzweck des Berichtsservices vor seiner Nutzung flexibel definiert werden.

\section{Schritt 5: Anpassung der Aktivitätsdiagramme}

Nachdem die Identifikation und die Auswahl geeigneter Vorgehensweisen abgeschlossen sind, müssen im nächsten Schritt die bestehenden Aktivitätsdiagramme angepasst werden. Eine Anpassung wird durchgeführt, indem die existierenden Aktivitäten durch die ,neuen“ Aktionen, denen jetzt eine Vorgehensweise zugeordnet ist, ersetzt werden. Dies trifft auf die Aktivitäten mit dem $\mathrm{Zu}$ stand 1.2, 1.3.1, 1.3.2.1 und 1.3.2.2 zu.

Nachdem die neuen Aktvitäten ins Aktivitätsdiagramm überführt wurden, erfolgt eine erneute Überprüfung der bereits vorgenommenen Serviceidentifikation. Zu prüfen ist, ob die neuen Aktivitäten, die die bisherigen Aktionen ersetzt haben, zu einem Service gekapselt oder als separate Dienste ausgeführt werden sollen. Zur Beantwortung dieser Frage lässt sich die in Abschnitt 5.2.2.2.1 präsentierte Vorgehensweise heranziehen. Dabei ist auch bei den neuen Aktionen zu beachten, dass alle Aktivitäten, die gemeinsam auftreten, zu einem Service zusammengefasst werden können.

Eine Besonderheit ergibt sich bei der Identifikation von Services, denen mehrere nicht dominierte Vorgehensweisen zugrunde liegen (Zustand 1.3.2.2). In diesem Zusammenhang ist darauf hinzuweisen, dass es nicht erforderlich ist, für jede Vorgehensweise einen eigenen Service zu entwickeln. Stattdessen stellt die Auswahllogik, die in diesem Service implementiert werden muss, sicher, welche Vorgehensweise bei der Ausführung des jeweiligen Services anzuwenden ist, so dass alle Vorgehensweisen in diesem Service implementiert werden können.

\subsection{Zusammenfassung und Würdigung des Vorgehensmodells}

Die Ausführungen dieses Kapitels hatten zum Ziel, ein Vorgehensmodell zu präsentieren, das sich zur konzeptionellen Gestaltung von SOBP eignet. Ausgehend von der Vorstellung und Bewertung verschiedener Vorgehensstrategien zur Gestaltung Serviceorientierter Anwendungen wurden mit dem Meet-in-themiddle-Ansatz hierbei eine Strategie identifiziert, die sich zur konzeptionellen Gestaltung von SOBP grundsätzlich einsetzen lässt. Kennzeichnend für diese Vorgehensweise ist, dass sie mit der Berichtsprozessanalyse (Abschnitt 5.2.1) gemäß der Top-down-Vorgehensweise beginnt. Im Rahmen der berichtsprozessmodellbasierten Serviceidentifikation (Abschnitt 5.2.2) erfolgt eine Erweiterung der prozessorientierten Top-down-Vorgehensweise um die Bottom-upSicht. Als letzter Schritt ist die fachliche Berichtsservicegestaltung durchzuführen (Abschnitt 5.2.3). 
Im Vergleich zu alternativen Vorgehensweisen zeichnet sich der in diesem Kapitel präsentierte Ansatz dadurch aus, dass bei der konzeptionellen Gestaltung von SOBP nicht nur methodische Aspekte berücksichtigt werden, die die prozessmodellorientierte sowie fachliche Identifikation und Gestaltung der Berichtsservices betreffen, sondern zusätzlich Gesichtspunkte aufgegriffen werden, die aktuell im Kontext der Governance Serviceorientierter Anwendungen diskutiert werden. ${ }^{798} \mathrm{Zu}$ nennen ist in diesem Zusammenhang vor allem die Einbeziehung einer Lebenszyklusbetrachtung der Berichtsservices sowie die Integration eines Vorgehens zur Beurteilung der Dienstgüteeigenschaften. 799

Die Einbindung einer Lebenszyklusbetrachtung sowie einer Methodik zur Bewertung der Dienstgüte der Services ermöglicht es, bei der konzeptionellen Gestaltung von Berichtsservices Einfluss auf die Anzahl und damit Pflege der in einem Serviceverzeichnis registrierten Dienste zu nehmen. Angesichts der Vielzahl der von einer Unternehmung betriebenen Berichtsanwendungen, Informationsflüsse und somit potenziell in Frage kommenden Berichtsservices stellt die Pflege des Serviceverzeichnisses eine wichtige Herausforderung dar. Hierbei bietet die frühzeitige Prüfung der Potenziale der Nutzung unternehmungsexterner Services die Möglichkeit, einerseits aufwendige Eigenentwicklungen zu verringern und andererseits einen Mehrwert bei der Informationsversorgung der Entscheidungsträger durch die Bereitstellung zusätzlicher Informationen zu schaffen.

Ferner lässt sich durch diese Instrumente gewährleisten, dass die Berichtsservices in der von den Nutzern geforderten Qualität ausgeführt werden. Da sich Berichtsprozesse und die damit verbundene effiziente und effektive Bereitstellung verlässlicher Geschäfts- und Finanzinformationen unmittelbar auf die Leistungsfähigkeit der Unternehmungssteuerung auswirken, ist die Einhaltung der Qualitätskriterien sowohl bei der Nutzung unternehmungsexterner Services als auch bei der Gestaltung neuer (interner) Berichtsservices umso mehr von Bedeutung. Mit Blick auf die aktuelle SOA-Literatur findet dieser Aspekt bei der Gestaltung Serviceorientierter Geschäftsprozesse bisher eine nur untergeordnete Rolle.

Um zu gewährleisten, dass die zu gestaltenden SOBP dem Informationsbedarf der Berichtsempfänger genügen, wurde mit dem Ordnungsrahmen zur Zielbestimmung von SOBP ein Schema präsentiert, das alle wichtigen Gestaltungsparameter eines SOBP aufführt. ${ }^{800}$ Ein weiterer Vorteil des in diesem Kapitel präsentierten Vorgehensmodells ist in der Erweiterung der prozessmodellbasierten Vorgehensweise durch eine Bottom-up-gestützte Serviceidentifikation zu

798 Eine Zusammenstellung verschiedener Methoden zur Konzeption Serviceorientierter Anwendungen präsentiert Offermann (2008), S. 463f.

799 Siehe hierzu die Abschnitte 5.2.1.3 und 5.2.3.4.

800 Vgl. Abschnitt 5.2.1.1. 
sehen. Auf diese Weise lässt sich gewährleisten, dass die von den Berichtsanwendungen in vielfacher Ausprägung zur Verfügung gestellten Funktionen bei der konzeptionellen Gestaltung einer SOBP berücksichtigt werden und als potenzielle Berichtsservices bzw. Berichtsservicekandidaten in Frage kommen.

Wird das in diesem Kapitel präsentierte Vorgehen zur konzeptionellen Gestaltung von SOBP verfolgt, hat dies zur Konsequenz, dass im Rahmen der Berichtsprozessanalyse alle zur Verfügung stehenden Prozessmodelle betrachtet werden müssen. Folglich sind nicht nur die fachlichen, sondern auch die technischen Prozessmodelle bereits implementierter Berichtsprozesse bei der Berichtsprozessmodellanalyse einzubeziehen. Vor dem Hintergrund, dass Berichtsprozessmodelle als Kommunikationsinstrument von den bei der SOBPGestaltung beteiligten Fachabteilungen und Softwareentwickler genutzt werden, kann das Problem auftreten, dass unterschiedliche Prozessmodelle als Kommunikationsgrundlage zur Verfügung stehen, die zu einem uneinheitlichen Prozessverständnis führen. Der Grund hierfür liegt darin, dass die fachliche und technische Modellierung von Prozessen i. d. R. entkoppelt voneinander durchgeführt werden, so dass eine durchgängige Modellierung von der fachlichen bis zur technischen Ebene erschwert wird bzw. ausbleibt. ${ }^{801}$

Als Folge einer inkonsistenten Überführung der fachlichen Anforderungen der Services in die zum Einsatz kommenden IS lässt sich daher eine Modellierungs- sowie Dokumentationslücke identifizieren, die dadurch auftritt, dass Änderungen in den fachlichen Prozessmodellen meist nicht mit den technischen Prozessmodellen synchronisiert werden. Diese von THOMAS/LEYKING/DREIFUS als Top-down-Problem bezeichnete Herausforderung wirkt sich nicht auf den Entwurf, sondern auch auf den Betrieb einer SOA aus. ${ }^{802}$ Gleichzeitig lässt sich das Problem erkennen, dass notwendige Änderungen an den von einer Unternehmung betriebenen IS nicht oder nicht vollständig in den fachlichen Prozessmodellen dokumentiert werden. Hier kann von einem Bottom-up-Problem gesprochen werden.

Die von den Autoren angeführten Probleme lassen sich beheben, indem zu den in Abschnitt 5.2.1.2 aufgeführten Modellierungsmethoden alternative Notationen zur Prozessmodellierung eingesetzt werden, die sich als Kommunikationsinstrument zwischen den bei der SOBP-Entwicklung beteiligten Akteuren nutzen lassen. Einen geeigneten und derzeit viel diskutierten Ansatz zur Verringerung sowohl der Modellierungs- als auch der Dokumentationslücke liefert die Business-Process-Modeling-Notation (BPMN).

Mit Hilfe der BPMN lässt sich ein fachliches Prozessmodell in ein fachlichtechnisches Anwendungsmodell transformieren, das der Synchronisation und 
Integration der fachlichen und technischen Beschreibungsebene dient. ${ }^{803}$ Die BPMN stellt geeignete Sprachkonstrukte zur Verfügung, mit der das Grundgerüst für den Ablauf des Prozesses definiert werden kann. ${ }^{804}$ Als Ausgangspunkt dient die Ablauflogik, die in das fachliche Prozessmodell eingebettet ist. Die Erweiterung des fachlichen Prozessmodells erfolgt durch die Einbeziehung technischer Details zur Prozessausführung. ${ }^{805}$ Bei der Modellierung des fachlich-technischen Anwendungsmodells bedient sich die BPMN einer grafischen Darstellung der Prozesse und soll so dem Analysten, dem technischen Entwickler und dem Manager als gemeinsame Kommunikationsplattform dienen. 806 Damit repräsentiert die BPMN-Notation das Bindeglied zwischen dem Geschäftsprozessdesign und der Prozessimplementierung, wobei sie sich unabhängig von der späteren technischen Umsetzung bzw. Implementierung verwenden lässt.

803 Vgl. Thomas/Leyking/Dreifus (2007), S. 38.

804 Die BPMN wurde von der BusinEss Process MANAgEMENT INITIATIVE (BPMI) im Jahr 2002 entwickelt und 2006 von der OBJeCt MANAGEMENT Group (OMG) als Standard aufgenommen. 


\section{Zusammenfassung und Resümee}

In diesem Kapitel werden die zentralen Ergebnisse der vorliegenden Arbeit zusammengefasst. Hierzu führt Abschnitt 6.1 den Aufbau sowie die wichtigsten Erkenntnisse der jeweiligen Kapitel auf. Abschnitt 6.2 diskutiert die Potenziale des in dieser Arbeit präsentierten Architektur- und Vorgehensmodells, die sich für die Reduzierung der Problemfelder des Berichtswesens ergeben. Abschnitt 6.3 widmet sich einem Ausblick sowie weiterem Forschungsbedarf.

\subsection{Allgemeine Zusammenfassung}

Das Ergebnis dieser Arbeit ist ein Architektur- und Vorgehensmodell, das zur konzeptionellen Gestaltung von Berichtsprozessen auf Basis einer SOA und XBRL zum Einsatz kommen kann.

Die Ausführungen von Kapitel 2 hatten zum Ziel, mit der Prozessorientierung des betrieblichen Berichtswesens ein betriebswirtschaftliches Anwendungsfeld zu präsentieren, das vor dem Hintergrund einiger Problemfelder Verbesserungspotenziale bietet. Die Problemfelder verhindern sowohl eine effektive als auch effiziente Verarbeitung der im Rahmen des Berichtswesens anfallenden Geschäfts- und Finanzinformationen. Als zusammenfassendes Ergebnis der Ausführungen dieses Kapitels wurde das Berichtswesen einer Unternehmung als Gesamtheit aller Personen, Prozesse und IS aufgefasst, die zur Erzeugung, Aufbereitung, Übermittlung und Auswertung von formalisierten Berichten beitragen. Als Kernprozesse wurden die Informationsbeschaffung, Berichtserstellung, Berichtsdistribution und Berichtsaufnahme identifiziert.

Aus der Perspektive der Wirtschaftsinformatik stellt sich die Frage, welche Konzepte und Technologien sich zur Lösung oder Reduzierung betriebswirtschaftlicher Probleme einsetzen lassen. Mit dem Konzept einer SOA liegt ein in Wissenschaft und Praxis aktuell viel diskutierter Ansatz vor. Kapitel 3 hatte zum Ziel, aus den Beiträgen des Themenbereichs SOA ein integriertes SOA-Verständnis abzuleiten. Als Ergebnis ließ sich folgende Definition ermitteln: „Unter einer SOA wird ein abstraktes Architekturkonzept aufgefasst, bei dem wiederverwendbare Softwarekomponenten, die bestimmten Designprinzipien unterliegen und eine gezielte Funktion erfüllen, zur Unterstützung einer integrierten Informationsverarbeitung zum Einsatz kommen und insbesondere zur Flexibilisierung und/oder Automatisierung von Geschäftsprozessen beitragen sollen."

Zusammenfassend ist festzuhalten, dass es sich bei SOA um ein ganzheitliches Architekturkonzept zur Gestaltung und Integration von IS handelt, das das Ziel verfolgt, eine integrierte Informationsverarbeitung zu ermöglichen. Kenn- 
zeichnend für SOA ist, dass bei diesem Architekturkonzept nicht die Orientierung an den Organisationseinheiten sowie an den von diesen betriebenen monolithischen, heterogenen IS im Vordergrund steht, sondern die Nutzung kleiner, zeitlich stabiler Prozess- und Funktionsbausteine favorisiert wird.807 Die Bausteine werden innerhalb einer SOA als lose gekoppelte, standardisierte (Software-)Services bzw. (Software-)Dienste angeboten, die sich zu einem funktional erweiterbaren IS kombinieren lassen.

Damit verspricht der Betrieb einer SOA, eine agile Applikationslandschaft zu realisieren, die an unternehmungsindividuelle Geschäftsanforderungen angepasst werden kann und sich im Vergleich zu alternativen Integrationsansätzen durch ein höheres Maß an Flexibilität und Geschwindigkeit auszeichnet. ${ }^{808}$ Mit Blick auf die fachlichen Nutzenpotenziale erweist sich das sowohl von der Wissenschaft als auch von der Praxis vorgebrachte Versprechen, applikationsund/oder unternehmungsübergreifende Geschäftsprozesse durch die Verwendung orchestrierter Services zu flexibilisieren und/oder automatisieren, als wichtiger Treiber für das Interesse an SOA. Im Gegensatz zu monolithischen Anwendungen stellt der Ansatz einer SOA damit in Aussicht, prozessorientierte Anwendungen $\mathrm{zu}$ realisieren, die sich nicht isoliert voneinander betreiben lassen, sondern sich zum Ausführungszeitpunkt auf Basis dynamischer Services zusammensetzen. ${ }^{809}$ Vor diesem Hintergrund und der festzustellenden Defizite bei der Ausführung von Berichtsprozessen stellt SOA einen interessanten Ansatz für die Zwecke des Berichtswesens dar.

Kapitel 4 widmete sich der Vorstellung eines Architekturmodells, das für die Gestaltung von SOBP zum Einsatz kommen kann. Hierzu war es erforderlich, den SOBP-Begriff zu konkretisieren. Die dieser Arbeit zugrunde liegende SOBP-Definition lautet: „Unter einem SOBP wird ein Berichtsprozess verstanden, bei dem die Aktivitäten und Teilprozesse der Kernprozesse Informationsbeschaffung, Berichtserstellung, Berichtsdistribution und Berichtsaufnahme mit Hilfe von Berichtsservices implementiert werden, die dem Serviceverständnis einer SOA sowie den zugrunde liegenden Designprinzipien der Serviceorientierung folgen." Als Ergebnis ausgewerteter Vorschläge wurde zunächst ein Architekturmodell präsentiert, das sich zur Gestaltung bzw. Einführung einer SOA einsetzen lässt. Im Anschluss erfolgte eine Erweiterung des Architekturmodells, die zum Ziel hat, eine stärkere semantische Verarbeitung von Geschäfts- und Finanzinformationen zu ermöglichen. Die Erweiterung basiert auf dem XBRLStandard.

807

808 Dass die Serviceorientierung einen größeren Beitrag zur Agilität einer Unternehmung als einzelne Technologien leistet, ließ sich in verschiedenen Fallstudien belegen. Vgl. Schelp/Winter (2007), S. 44.

Vgl. Winkler (2007), S. 258. 
Die Ausführungen des Kapitels 5 fokussierten ein Vorgehensmodell zur konzeptionellen Gestaltung von SOBP. Aufbauend auf einer Diskussion bestehender Vorgehensstrategien, die generell für die Gestaltung Serviceorientierter Anwendungen in Frage kommen, wurde der Meet-in-the-middle-Ansatz, der sowohl Aspekte des Top-down- als auch des Bottom-up-Vorgehens integriert, als geeigneter Ansatz identifiziert.

\subsection{Zusammenfassung der Nutzenpotenziale des Architektur- und Vor- gehensmodells zur Reduzierung der Defizite beim Reporting}

Die folgenden Ausführungen fassen die zentralen Erkenntnisse dieser Arbeit zusammen im Hinblick auf die Möglichkeit, die in Abschnitt 2.3 diskutierten Defizite bei der Ausführung der Kernprozesse des Berichtswesens zu reduzieren. In diesem Zusammenhang werden auch die Nutzenpotenziale erörtert, die sich aus dem um XBRL erweiterten SOA-Architekturmodell (Kapitel 4) sowie dem Vorgehensmodell zur konzeptionellen Gestaltung von SOBP (Kapitel 5) identifizieren lassen. Nachdem in Abschnitt 6.2.1 die Nutzenpotenziale zur Reduzierung zweckbezogener Defizite diskutiert werden, widmet sich Abschnitt 6.2.2 den Nutzenpotenzialen zur Minderung inhaltsbezogener Defizite. Die Ausführungen des Abschnitts 6.2.3 richten sich auf die Vermeidung syntaxbezogener Defizite, die beim Reporting auftreten können.

\subsubsection{Nutzenpotenziale zur Vermeidung zweckbezogener Defizite}

Die im Architekturmodell von Kapitel 4 dargestellte Möglichkeit, Anwendungssysteme auf Basis von Services und XBRL zu koppeln, birgt nicht nur Potenziale, die sich auf die Effizienz der Informationsverarbeitung im betrieblichen Berichtswesen auswirken. ${ }^{810}$ Die Vorteile, die sich aus den beschriebenen Möglichkeiten zur semantischen sowie datenkonsistenten Verarbeitung der Geschäfts- und Finanzinformationen ergeben, haben unmittelbaren Einfluss auf die fachlichen Nutzungsmöglichkeiten einer SOA für die Zwecke des Berichtswesens, die sich wiederum auf die Effektivität des Reportings auswirken.

Der integrierte Einsatz von Services und XBRL eröffnet Möglichkeiten, die Empfängerorientierung und die Aktualität der Informationsversorgung im betrieblichen Berichtswesen zu steigern. Die Gestaltung geeigneter, auf den Informationsbedarf der Berichtsempfänger zugeschnittener Services, die ausgewählte Daten der XBRL-Instanzdokumente verarbeiten, ermöglicht es, eine empfängerorientierte Informationsversorgung zu implementieren. Indem $\mathrm{Be}-$ richtsservices realisiert werden können, die eine bestimmte Sicht auf einen Da-

810 Siehe hierzu die Ausführungen in Abschnitt 6.2.3. 
tenbestand abbilden, lässt sich gewährleisten, dass nur diejenigen Informationen eines Instanzdokuments einem Berichtsempfänger zur Verfügung gestellt werden, die für diesen eine Relevanz besitzen.

Eine stärkere Empfängerorientierung lässt sich zudem durch die verschiedenen Publikationsmöglichkeiten realisieren, die der Einsatz von XBRL bietet. Durch die Anwendung von Cascading Style Sheets (CSS) oder der Extensible Stylesheet Language (XSL) lassen sich XBRL-Berichte nach den Anforderungen der Berichtsempfänger layouten und in verschiedenen Formaten und Präsentationsvarianten beispielsweise auf der Web-Site einer Unternehmung oder im Intranet zum Download anbieten. ${ }^{811}$ Darüber hinaus lassen sich die Informationen dieser Dokumente durch den Einsatz z. B. von Web Services, die ausgewählte Informationen der veröffentlichten Dokumente abfragen, weiterverarbeiten.

\subsubsection{Nutzenpotenziale zur Minderung inhaltsbezogener Defizite}

Mit Blick auf die in Abschnitt 2.3.2 thematisierten inhaltsbezogenen Problemfelder, die eine effektive Ausführung der Berichtsprozesse verhindern, ist zu konstatieren, dass insbesondere der Einsatz von XBRL Nutzenpotenziale zur Reduzierung dieser Problemfelder bietet.

Eine wichtige Aufgabe erfüllen in diesem Zusammenhang die XBRLTaxonomien, die in dem in Kapitel 4 präsentierten Architekturmodell in einer separaten Schicht angeordnet sind. Als technische Referenz für die zum Einsatz kommenden Instanzdokumente gewährleisten die Taxonomien, dass sich nur vordefinierte Elemente bzw. Konzepte mit vereinbarten Attributen als Informationseinheiten innerhalb einer SOBP nutzen lassen. Da es sich bei den Taxonomien um eine normierte Begriffs- und Strukturvorschrift handelt, erhalten die Geschäfts- und Finanzinformationen, die in den Instanzdokumenten mit Hilfe der Taxonomieelemente beschrieben werden, eine Bedeutung. Auf diese Weise lassen sich die in einem Instanzdokument präsentierten Informationen durch den Einsatz entsprechender Berichtsservices nicht nur in weitere Berichtsanwendungen transferieren, sondern auch von menschlichen Nutzern interpretieren.

Im Zusammenhang mit der Nutzung von XBRL ist hervorzuheben, dass es sich bei diesem XML-Dialekt um einen ausschließlich technischen Standard handelt, dessen Ziel es ist, über ein von allen Nutzern geteiltes sowie eindeutiges Verständnis zu den Informationsobjekten des Berichtswesens Geschäfts-

811 Vgl. Nutz/Strauß (2002), S. 452. 
und Finanzinformationen automatisiert zu verarbeiten. ${ }^{812}$ Folglich geht es bei XBRL um die Abbildung bestehender Rechnungslegungsvorschriften und keineswegs um die Erstellung neuer inhaltlicher Rechnungslegungsstandards. ${ }^{813}$ Damit besitzt XBRL nicht das Potenzial, Probleme zu lösen, die sich beispielsweise aus der Interpretation sowie aus dem Vergleich von Bilanzwerten verschiedener Rechnungslegungsstandards ergeben.

Bei einem Blick auf die vergangenen sowie aktuellen Aktivitäten zur Etablierung von XBRL wird deutlich, dass dieser Standard zur Verarbeitung von Geschäfts- und Finanzinformationen eine große Bedeutung hat. ${ }^{814}$ Nach WILLIS/ HANNON ist in den kommenden Jahren eine stärkere Einbettung einer Funktionalität zur Verarbeitung von XBRL-Daten in die bestehenden Softwareprodukte zu erwarten. ${ }^{815}$ Eine zunehmende Nutzung von XBRL, die teilweise auf gesetzlichen Vorgaben beruht, ist gegenwärtig an ausländischen Börsenplätzen sowie Börsenaufsichten wie der Shanghai Stock Exchange (SSE), der Comisión Nacional del Mercado de Valores (CNMV), dem Committee of European Banking Supervisors (CEBS) oder der amerikanischen Bankenaufsicht festzustellen. ${ }^{816}$ Im internationalen Vergleich ist die Zahl der in Deutschland publizierten Jahresabschlüsse auf Basis von XBRL bis zum gegenwärtigen Zeitpunkt als gering einzustufen. Als Pioniere in diesem Bereich gelten die Unternehmungen Software AG, Fraport und Microsoft, die ausgewählte Geschäftszahlen im XBRLFormat an der Deutschen Börse veröffentlicht haben. ${ }^{817}$

\subsubsection{Nutzenpotenziale zur Reduzierung syntaxbezogener Defizite}

Der Einsatz von Berichtsservices, die gekapselte Funktionen zur Verfügung stellen, bietet die Möglichkeit, Geschäfts- und Finanzinformationen über Applikationsgrenzen hinweg zu verarbeiten. Auf diese Weise lassen sich syntaktisch bedingte Problemfelder reduzieren, die oftmals dadurch entstehen, dass diese Informationen bei der Ausführung der Berichtsprozesse zwischen verschiede-

812 Vgl. Baars (2005), S. 194. Dass es sich bei XBRL um ein technisches Framework handelt, wird auch bei RAMIN/KeSSELMEYER/OTT deutlich. Vgl. Ramin/Kesselmeyer/Ott (2006a), S. 181.

813 Vgl. Meyer-Pries/Gröner (2002), S. 45.

814 Hannon präsentiert hierzu einen Überblick zu den in den vergangenen Jahren initiierten Aktivitäten zur Weiterentwicklung sowie zur Verbreitung von XBRL. Vgl. Hannon (2004), S. 58.

815 Vgl. Willis/Hannon (2005), S. 59.

$816 \mathrm{Vgl}$. Ramin/Kesselmeyer/Ott (2006a), S. 180f. Einen Überblick über weitere XBRLProjekte liefert auch Hannon (2006), S. 59.

817 Vgl. Gruppe Deutsche Börse (2009). Als weitere Pioniere lassen sich auch die Unternehmungen MORGAN STANLEY und REUTERS nennen. Vgl. Kranich/Schmitz (2003), S. 79. 
nen IS ausgetauscht und von verschiedenen Anwendern bearbeitet werden müssen, die für die Analyse und Darstellung der Berichte eine Reihe von SoftwareTools und Dateiformate nutzen. ${ }^{818}$ Dass sich Geschäfts- und Finanzinformationen über Systemgrenzen hinweg durch den Einsatz entsprechender Berichtsservices verarbeiten lassen, wird durch das Prinzip der „Abstraktion von der Implementierungslogik" gewährleistet. ${ }^{819}$

Da gemäß des Prinzips der „Abstraktion von der Implementierungslogik“ lediglich die Serviceschnittstelle für die Servicenutzung ausreicht, besteht die Möglichkeit, gekapselte Dienste nicht nur zum Ablauf verschiedener Geschäftsprozesse schnell und ohne zusätzlichen Aufwand nutzen zu können, 820 sondern mit selbigen Vorteilen für die Gestaltung einer SOBP verwenden zu können. Kostenreduzierende Effekte ergeben sich dabei infolge einer einfachen Anpassung der Berichtsprozesse, ${ }^{821}$ da Änderungen in den zu unterstützenden Prozessen oder auch Teilprozessen durch eine Neu- bzw. Rekombination der Services und nicht durch eine Neuentwicklung oder einer vollständigen Anpassung der bestehenden Funktionalität der Dienste durchgeführt werden können. 822 Als Beispiel lässt sich der Einsatz eines Berichtsservice zur Währungsumrechnung nennen, der aufgrund seines Funktionsumfangs in vielen Berichtsprozessen verwendet werden kann, bei denen zur Ermittlung eines bestimmten Ergebnisses eine Währungskonvertierung erforderlich ist.

Die beim Betrieb einer SOA anvisierten Einsparungspotenziale, die sich aus einer hohen Wiederverwendung der Berichtsservices ergeben, sind insbesondere dann gegeben, wenn die Dienste nicht nur von einem großen Nutzerkreis, sondern auch in möglichst vielen Berichtsprozessen zum Einsatz kommen. Hierzu wurde in Kapitel 5 ein Vorgehensmodell präsentiert, das zum Ziel hat, Berichtsservices mit einem hohen Wiederverwendungsgrad konzeptionell zu gestalten.

Der Einsatz von Berichtsservices, die aus den Aktivitäten eines zugrunde liegenden fachlichen Berichtsprozessmodells gebildet und mit Hilfe einer Workflow-Engine orchestriert werden, birgt zudem Potenziale, eine applikations- und/oder unternehmungsübergreifende SOBP zu flexibilisieren und/oder zu automatisieren. Medienbrüche, fehlerhafte Eingaben sowie unproduktive Datentransformationen lassen sich auf diese Weise vermeiden.

818 Vgl. Abschnitt 2.3.3.

819 Vgl. hierzu Abschnitte 3.3.2.1.

820 Vgl. hierzu Winkler (2007), S. 258.

821 Wie SCHELP/WINTER in diesem Zusammenhang feststellen, erfordert eine flexible Organisation sowie Reorganisation der zu unterstützenden Geschäftsprozesse auf Basis modularer Services generell, dass die Applikationen ressourcenschonend integriert und entkoppelt werden können. Vgl. Schelp/Winter (2007), S. 44. 
Dabei können verschiedene Operationen Bestandteil eines automatisierten und flexibel anpassbaren Berichtsprozesses sein. ${ }^{823}$ Berichtsservices können z. B. sequentiell entlang einer vorgegebenen Anweisungskette ausgeführt werden. In diesem Zusammenhang eröffnet die Nutzung von Services zahlreiche Einsatzszenarien, bei denen formulierte Datenabfragen kaskadierend weitere Datenabfragen (d. h. weitere Services) entlang einer Prozesskette auslösen. Neben reinen Datenabfragen ist auch die Abbildung komplexer Prozesse vorstellbar, bei denen beispielsweise Services eingebunden werden, die ein automatisches Benchmarking oder ein Kundenrating in einem SOBP integrieren. ${ }^{824}$ Eine derartige Abarbeitung sequentiell angeordneter Schritte lässt sich erweitern, indem bedingte Ausdrücke aufgenommen werden. Hierbei lassen sich alle aus den Programmiersprachen bekannten Ausprägungen einer Bedingung (wie einfache, zweifache oder mehrfache Alternativen) zur Abbildung einer entsprechenden Prozesslogik einsetzen.

Über den Einsatz von Diensten lassen sich zudem Berichtsprozesse ausführen, die zu einem im Vorfeld festgelegten Zeitpunkt ausgelöst werden. In diesem Zusammenhang ist das Beispiel einer Servicegestützten Bereitstellung von Informationen zu nennen, die nach vordefinierten Zeitintervallen automatisiert in ein Ziel-IS eingelesen werden. In Abhängigkeit des spezifischen Anwendungsbereichs wäre hier eine minütliche, stündliche, tagesaktuelle, wöchentliche oder auch monatliche Informationsbereitstellung denkbar. Eine derartige Anwendung von Services, die sich zu einem zeitgesteuerten Prozessservice orchestrieren lassen, würde damit Aufgaben bzw. Funktionen übernehmen, die im Kontext eines DWH-Einsatzes klassischerweise von ETL-Systemen erfüllt werden. 825

Genauso wie bei der zeitgesteuerten Auslösung eines Berichtsprozesses kann eine Prozessautomatisierung auch durch das Eintreten eines vordefinierten Ereignisses bzw. Events erfolgen. Eine derartige Funktionalität wird im Kontext von Datenbanksystemen auch als Trigger bezeichnet. Als Beispiel für einen ereignisgesteuerten Prozess ist ein Service denkbar, der nach der Änderung eines Wertes eines bestimmten Attributs einen weiteren Dienst auslöst. Durch diesen wiederum lässt sich eine bestimmte Abfrage beispielsweise auf die Relation eines weiteren Datenbanksystems ausführen, bevor die hieraus resultierende Ergebnismenge schließlich in eine separate Tabelle geschrieben wird. 


\subsection{Ausblick und weiterer Forschungsbedarf}

Mit Blick auf die zum Teil euphorische Erwartungshaltung, die seitens einiger Autoren im Zusammenhang mit dem Betrieb einer SOA geäußert wurde, ${ }^{826}$ verspricht der Einsatz von Web Services generell Nutzenpotenziale für die kostengünstige Integration von Anwendungen bei gleichzeitiger Unterstützung unternehmungs- und/oder applikationsübergreifender Geschäftsprozesse. ${ }^{827} \mathrm{Da}$ durch, dass sie eine klar definierte Aufgabe erfüllen, bieten Web Services die Möglichkeit, die von ihnen angebotene Leistung zu zentralisieren und auszulagern. ${ }^{828}$ Damit eignen sich Web Services auch zur Ausführung von Berichtsservices, die die Verarbeitung von Geschäfts- und Finanzinformationen fokussieren und von einer großen Anwenderzahl in Anspruch genommen werden können. Im Vergleich zu konkurrierenden Ansätzen bieten Web Services den Vorteil einer schnelleren und kostengünstigeren Nutzung. ${ }^{829}$ Damit besitzt dieser Standard insbesondere eine Relevanz für kleine und mittlere Unternehmungen (KMU), die vor dem Hintergrund einer geringeren Ressourcenausstattung an kostengünstigen Integrationslösungen interessiert sind. 830

ALT/HEUTSCHI/ÖSTERLE prophezeien in diesem Zusammenhang, dass Web Services insbesondere für unternehmungsinterne Zwecke sowie bei bereits bestehenden Unternehmungspartnerschaften eingesetzt werden.831 Damit besitzt diese Technologie Nutzungspotenziale für die Gestaltung von Berichtsprozessen von und zwischen Unternehmungen, die beispielsweise innerhalb einer Konzernstruktur miteinander verbunden sind.

Im Ergebnis ist zu konstatieren, dass es bisher nur in wenigen Ausnahmefällen gelungen ist, eine unternehmungsweite SOA zu implementieren, innerhalb dieser sämtliche unternehmungsinterne und -übergreifende Geschäftsprozesse

Vgl. Abschnitt 3.2.

IssING prophezeit in diesem Zusammenhang, dass sich die Realisierung von Web Service-basierten Architekturen zu einem Mainstream entwickeln wird, wobei nach Auffassung des Autors positive Auswirkungen auf die Effizienz einer SOA in belegbarer Weise durch den Einsatz von Investitionsrechnungen zu erwarten sind. Vgl. Issing (2003), S. 30. Der Autor lässt in diesem Zusammenhang allerdings offen, auf welche Weise eine Quantifizierung der Effizienzvorteile - z. B. ausgedrückt in Form eines positiven Kapitalwerts - durchgeführt werden kann.

$828 \mathrm{Vgl}$. Alt/Heutschi/Österle (2003), S. 64.

829 Vgl. Küster (2003), S. 6.

830 Die Vorteile, die sich aus der Interoperabilität insbesondere für KMU ergeben, werden prägnant von KÜSTER zusammengefasst, der in diesem Zusammenhang schreibt: „WebServices bringen Interoperabilität für die Massen“.

$831 \mathrm{Vgl}$. Alt/Heutschi/Österle (2003), S. 69. 
auf Basis verknüpfter Services automatisiert und/oder flexibilisiert sind. 832 Stattdessen wurden in der Vergangenheit Projekte mit dem Ziel initiiert, einzelne Anwendungsbereiche bzw. Geschäftsprozesse einer Unternehmung durch die Nutzung ausgewählter Services zu unterstützten, so dass die daraus resultierenden softwaretechnischen Lösungen den Charakter einer Serviceorientierten Anwendung oder Plattform aufweisen. ${ }^{833}$ Als federführende Branchen, die sich in den vergangenen Jahren intensiv mit der Einführung von SOA beschäftigt haben, sind der Finanz- und der Logistiksektor zu nennen. 834

Der Einschätzung von RIEKS zufolge ist generell die augenblickliche Nutzung von SOA mit der zögerlichen Anwendung des Internets Mitte der 90er Jahre vergleichbar. ${ }^{835}$ Ein Blick auf die Vielzahl aktueller Beiträge und initiierter Integrationsprojekte verdeutlicht, dass das Interesse an SOA nach wie vor ungebrochen ist, so dass SOA den befürchteten Status eines neuen Schlagwortes in der Wirtschaftsinformatik hinter sich gelassen hat. ${ }^{836}$ Gleichzeitig ist aber auch zu bemerken, dass das Wissen zur umfassenden Einführung einer SOA insbesondere in den Fachabteilungen vieler Unternehmungen defizitär ist. 837 Vor dem Hintergrund der nach wie vor anhaltenden Aktualität im Zusammenhang mit der Entwicklung und Nutzung von Services sind daher weitere Forschungsanstrengungen zu erwarten, so dass SOA auch in den kommenden Jahren die Diskussion um geeignete Konzepte zur IT-Unterstützung einer integrierten Informationsverarbeitung beherrschen wird. ${ }^{838}$ Folglich ist für die weitere

832 Auf die bisher geringe Anzahl von SOA-Implementierungen und den damit verbundenen geringen Erfahrungsschatz mit SOA verweisen auch BUXMANN/HESS/WIDJAJA. Vgl. Buxmann/Hess/Widjaja (2007), S. 1316. Nach STREIBICH liegt in Deutschland der derzeitige Anteil von SOA-Projekten im Verhältnis zur Gesamtheit aller Integrationsprojekte auf einem niedrigen Niveau von ca. $15 \%$. Vgl. Streibich (2008), S. 73.

833 Vgl. hierzu die Beispiele einer Serviceorientierten Anwendung bei Hinkelmann/ Thönssen/Probst (2005), S. 356ff.; Modjo Kamneng et al. (2007), S. 289ff.; Theling/ Janssen/Loos (2006), S. 403ff.; Thomas/Leyking/Dreifus (2007), S. 39ff.; Luhmann/Meister/Wulff (2007), S. 344ff.; Hanhart/Legner/Österle (2008), S. 478ff.; Kubli et al. (2008), S. 544ff.; Schelp/Aier (2008), S. 535ff.

834 Vgl. Streibich (2008), S. 73. Prominente Beispiele von Unternehmungen, die mit SOA positive Erfahrungen gesammelt haben, sind die DeUTSCHE POST und Credit SuISSE. Vgl. Esch et al. (2008), S. 93.

835 Vgl. Rieks (2006), S. 6.

836 Der Einschätzung des Analystenhauses GARTNER zufolge werden bis 2010 rund $65 \%$ der großen Unternehmungen über $35 \%$ ihres Anwendungsportfolios mit Hilfe einer SOA realisieren. Vgl. Thomas/Leyking/Dreifus (2007), S. 44.

837 Vgl. Streibich (2008), S. 73.

838 Mit Blick auf den GARTNER HYPE CYCLE - eine von der Unternehmung GARTNER veröffentlichte Kurve, die für die kommenden zehn Jahre die wichtigsten Technologien prognostiziert, die bei der Anwendungsentwicklung zum Einsatz kommen - wird SOA für die kommenden zwei bis fünf Jahren herausragen. Vgl. Pütter (2008), S. 2. 
Akzeptanz des SOA-Konzepts zu fordern, dass die Nutzungspotenziale Serviceorientierter Anwendungen und ihre Implikationen für die Geschäftsmodelle von Softwareanbietern und -nachfragern in den jeweiligen Arbeiten, die sich mit diesem Aspekt beschäftigen, besser ausgearbeitet werden. ${ }^{839}$

Ein weiteres wichtiges Forschungsfeld umfasst die Weiterentwicklung und den Einsatz von SOA-Governance-Technologien, um die Einhaltung von SLA zu gewährleisten, den Lebenszyklus von Services zu steuern oder die Verwaltung der Benutzerprofile in einer SOA zu ermöglichen. ${ }^{840}$ Die Vorteile, die eine Nutzung von Berichtsservices zur Flexibilisierung und/oder Automatisierung von SOBP bieten, können sich nämlich umkehren, wenn der Aufwand zur Bewältigung der daraus resultierenden Servicekomplexität die Potenziale übersteigt, die sich durch die Flexibilisierung und/oder Automatisierung einer derartigen Lieferkette eröffnen. Die Gefahr einer steigenden Servicekomplexität ist insbesondere vor dem Hintergrund nachvollziehbar, wenn davon ausgegangen wird, dass die bereits im Einsatz befindlichen Services im Fall einer notwendig gewordenen Anpassung entweder von der SOA-betreibenden Unternehmung nachträglich geändert oder durch neue Dienste, die von einem unternehmungsexternen Anbieter stammen, ersetzt werden.

Die zu bewältigende Komplexität resultiert insbesondere aus den Abhängigkeiten zwischen den Diensten, wie sie im Rahmen der Orchestrierung von Services angestrebt werden. Wird ein Service in seinem Funktionsumfang modifiziert, sind die daraus resultierenden Auswirkungen auch an den abhängigen Diensten zu berücksichtigen. ${ }^{841}$ Bestehen vielfältige, in Teilen gar netzwerkartige Verknüpfungen zwischen den Services, sind von der SOA-betreibenden Unternehmung entsprechende Aufwendungen zu leisten, um eine Koordination sowie Überwachung der Servicenutzung zu ermöglichen.

Der Änderungsaufwand entsteht dadurch, dass die Serviceimplementierung im Fall einer notwendig gewordenen Änderung modifiziert werden muss. Sind von diesem Service weitere Dienste betroffen, so sind auch die von diesen erbrachten Funktionen zu überdenken und gegebenenfalls anzupassen. Ein wichtiger Kostentreiber bei der Änderung der Funktionalität eines Dienstes ist in diesem Zusammenhang in der Anpassung der Servicebeschreibungen zu identifizieren, deren Änderungsaufwand nach NEWCOMER/LOMOW im Vergleich zu den Aufwendungen, die bei der technischen Modifizierung eines Service entstehen, als höher einzustufen ist. ${ }^{842}$ Der bei der Anpassung einer Servicebeschreibung resultierende höhere Aufwand hängt insbesondere davon ab, inwie-

839 Dass dieser Zustand aktuell nicht gegeben ist, beklagen Nüttgens/Dirik (2008), S. 31 .

Vgl. hierzu Pütter (2008), S. 2.

841 Vgl. Schelp (2007), S. 146.

842 Vgl. Newcomer/Lomow (2005), S. 77. 
weit der $\mathrm{zu}$ ändernde Service als Leistungserbringer für weitere Dienste dient. ${ }^{843}$

Der in der Literatur vorgeschlagene Ausweg, Varianten des von der Änderung betroffenen Services zu gestalten, birgt die Gefahr, dass die daraus resultierende hohe Anzahl der Dienste zum einen zu höheren Wartungskosten, zum anderen zu einer geringeren Wiederverwendungsrate der Services führen kann. Dieses Problem tritt insbesondere dann zutage, wenn neue Dienste oder neue Varianten bestehender Dienste in einen bereits existierenden Servicebestand aufgenommen werden.

Generell ist zu konstatieren, dass die Definition der Dienste i. d. R. sich als keine triviale Aufgabe erweist. SCHELP/STUTZ zufolge liegt der Aufwand, der unternommen werden muss, um einen Dienst mit einem hohen Wiederverwendungsgrad zu gestalten, im Vergleich zu Services, die sich durch einen niedrigeren Wiederverwendungsgrad auszeichnen, um mehr als das Dreifache höher. ${ }^{844}$ Vor diesem Hintergrund bietet der in Kapitel 5 vorgeschlagene Ansatz eine methodische Vorgehensweise, Berichtsservices mit dem Ziel einer hohen Wiederverwendbarkeit konzeptionell zu gestalten.

Ferner wird über die Akzeptanz einer SOA entscheiden, ob insbesondere für unternehmungskritische Anwendungen, bei denen beispielsweise vertrauliche Daten auf Basis orchestrierter Dienste verarbeitet werden, eine sichere Servicenutzung gewährleistet werden kann. ${ }^{845}$ Diese Überlegungen sind insbesondere für SOBP anzustellen, bei denen Berichtsservices zum Einsatz kommen, die als Web Services implementiert werden und damit allen Sicherheitsbedenken einer web-gestützten Informationsverarbeitung unterliegen.

Als Letztes ist zu bemerken, dass in dieser Arbeit der Themenbereich Serviceorientiertes Prozesscontrolling (SOPC) nicht thematisiert wurde. In diesem Zusammenhang ist zu konstatieren, dass mit der Berücksichtigung eines Bewertungsverfahrens zur Auswahl der zum Einsatz kommenden Berichtsservices das Fundament für ein SOPC gelegt wurde. Das Controlling Serviceorientierter Prozesse ist eine wichtige Voraussetzung für ihre Gestaltung. In der Erarbeitung geeigneter Instrumente zur Abschätzung der Wirtschaftlichkeit der mit Hilfe einer SOA ausgeführten Prozesse ist weiterer Forschungsbedarf zu identifizieren. ${ }^{846}$

843 Vgl. Newcomer/Lomow (2005), S. 77. Zur Beherrschung der Abhängigkeiten zwischen den Diensten und den damit verbunden Kosten schlagen die Autoren den Einsatz formaler Mechanismen zur Versionierung der Serviceverträge vor.

844 Vgl. Schelp/Stutz (2007), S. 67.

845 Auf die Notwendigkeit, Sicherheitsprobleme bei der Nachrichtenübertragung zwischen Services zu lösen, verweisen z. B. Beimborn/Mintert/Weitzel (2002), S. 278.

846 Eine auch für die Zwecke des Controllings von Berichtsservices geeignete Konzeption liefert vom Brocke (2008), S. 55ff. 
Trotz der Vielzahl der publizierten Beiträge ist ein Grund für die derzeit zu beobachtende Zurückhaltung bei der Implementierung von XBRL-gestützten Anwendungen in der Unkenntnis hinsichtlich der Funktionsweise und der Nutzungsmöglichkeiten von XBRL auszumachen. ${ }^{847}$ Über eine produktive Nutzung von XBRL entscheiden neben einem erforderlichen Fachwissen zu XBRL selbstverständlich Kosten-Nutzen-Überlegungen einer Unternehmung. Der Aufwand zur Implementierung einer XBRL-basierten Informationskette bestimmt sich insbesondere aus der Realisierung der Mapping-Prozesse zwischen den Objekten der Daten liefernden sowie Daten empfangenden Systeme und den Elementen einer Taxonomie. Als weiterer Kostentreiber fällt die fortwährende Anpassung der XBRL-Taxonomien aufgrund von Änderungen in den Rechnungslegungsstandards ins Gewicht. Auf der Nutzenseite stehen diesen Aspekten die stakeholderspezifische Bereitstellung von Geschäfts- und Finanzinformationen, die schnellere und verlässlichere Informationsversorgung sowie die Möglichkeit zum Online-Reporting entgegen.

Zusammen mit dem Einsatz auf den Informationsbedarf der Berichtsempfänger zugeschnittener Berichtsservices bietet der Einsatz von XBRL Potenziale zur Ausführung effizienter und effektiver Berichtsprozesse. Das in dieser Arbeit präsentierte Architektur- und Vorgehensmodell zeigt auf, wie sich XBRL und das SOA-Architekturkonzept für die konzeptionelle Gestaltung von SOBP kombinieren lassen. 


\section{Literaturverzeichnis}

Ackermann, Ulf; Eicker, Stefan; Neuhaus, Sebastian; Schuler, Peter (2006): Das EPA-Modell - Ein Referenzmodell für prozessorientierte, dienstbasierte Unternehmensarchitekturen, in: Lehner, Franz; Nösekabel, Holger; Kleinschmidt, Peter (Hrsg.): Multikonferenz Wirtschaftsinformatik 2006, Tagungsband 2, Berlin 2006, S. 183-197.

Aier, Stephan; Schönherr, Marten (2006): Status quo geschäftsprozessorientierter Architekturintegration, in: Wirtschaftsinformatik, 48. Jg., Heft 3, 2006, S. 188-197.

Aier, Stephan; Winter, Robert (2009): Virtuelle Entkopplung von fachlichen und IT-Strukturen für das IT/Business Alignment - Grundlagen, Architekturgestaltung und Umsetzung am Beispiel der Domänenbildung, in: Wirtschaftsinformatik, 51. Jg., Heft 1, 2009, S. 1-15.

Alt, Rainer; Heutschi, Roger; Österle, Hubert (2003): Web Services - Hype oder Lösung?, in: io new management, 71. Jg., Heft 1-2, 2003, S. 63-70.

Alves, Alexandre; Arkin, Assaf; Askary, Sid; Bloch, Ben; Curbera, Francisco; Goland, Yaron; Kartha, Neelakantan; Liu, Kevin; König, Dieter; Mehta, Vinkesh; Thatte, Satish; van der Rijn, Danny; Yendluri, Prasad; Yiu, Alex (Hrsg.) (2006): Web Services Business Process Execution Language Version 2.0: Committee Draft, 17.05.2006, http://www.oasisopen.org/committees/download.php/18714/wsbpel-specification-draftMay17.htm, 2006, abgerufen am 23.06.2009.

Amadee AG; Martin, Wolfgang (2007): SOA Check 2007: Trends im deutschen Markt, http://www.soa-check.net/Internet/SOACheck/Files/SOACheck 2007 Summary.pdf, Annecy 2007, abgerufen am 23.06.2009.

Andresen, Katja; Gronau, Norbert (2005): Die Gestaltung von Unternehmensarchitekturen - Wieviel Flexibilität ist notwendig?, in: ERP Management, 1. Jg., Heft 1, 2005, S. 30-32.

Andrews, Tony; Curbera, Francisco; Dholakia, Hitesh; Goland, Yaron; Klein, Johannes; Leymann, Frank; Liu, Kevin; Roller, Dieter; Smith, Dough; Thatte, Satish; Trickovic, Ivana; Weerawarana, Sanjiva (2003): Business Process Execution Language for Web Services (BPEL-WS), Version 1.1, 05.05.2003, http://download.boulder.ibm.com/ibmdl/pub/software/ dw/specs/ws-bpel/ws-bpel.pdf, 2003, abgerufen am 23.06.2009.

Baars, Henning (2005): XBRL, in: Controlling, 17. Jg., Heft 3, 2005, S. 193194. 
Ballinger, Keith; Brittenham, Peter; Malhotra, Ashok; Nagy, William A.; Pharies, Stefan (2001): Web Services Inspection Language (WS-Inspection) 1.0, http://download.boulder.ibm.com/ibmd1/pub/software/dw/specs/ws-wsil spec/ws-wsilspec.pdf, 2001, abgerufen am 23.06.2009.

Balzert, Heide (2000): Objektorientierung in 7 Tagen: Vom UML-Modell zur fertigen Web-Anwendung, Heidelberg/Berlin 2000.

Balzert, Helmut (2001): Lehrbuch der Software-Technik: SoftwareEntwicklung, Heidelberg/Berlin 2001.

Bange, Carsten (2004): Business Intelligence aus Kennzahlen und Dokumenten: Integration strukturierter und unstruktierter Daten in entscheidungsunterstützenden Informationssystemen, Hamburg 2004.

Bange, Carsten; Friedrich, Dirk (2008): BI im Mittelstand: Werkzeuge im Visier, in: BI Spektrum, 3. Jg., Heft 1, 2008, S. 34-35.

Bark, Michael; Fritsch, Werner (2006): Die richtigen SOA-Züge, in: InformationWeek, http://www.informationweek.de/showArticle.jhtml?articleID= 196603666\&cid=SOA, 2006, abgerufen am 23.06.2009.

Bauer, Andreas; Günzel, Holger (2004): Data Warhouse Systeme, Architektur, Entwicklung, Anwendung, Heidelberg 2004.

Bauler, Pascal; Feltz, Fernand; Biri, Nicolas; Pinheiro, Philippe (2007): Entwurf von serviceorientierten Architekturen auf Basis von Open-SourceSoftware, in: HMD - Praxis der Wirtschaftsinformatik, 44. Jg., Heft 253, 2007, S. 57-65.

Baumgartner, Heinz; Ebert, Klaus; Schleider, Karsten (o. J.): Regeln zur Modellierung von ereignisgesteuerten Prozessketten, Beilage zur kaufmännischen ZPG - Mitteilung Nr. 24, http://www.netzwerk-welt.de/common_files/ BWL/EPK.pdf, o. J., abgerufen am 23.06.2009.

Becker, Jörg; Knackstedt, Ralf (2004): Referenzmodellierung im DataWarehousing: State-of-the-Art und konfigurative Ansätze für die Fachkonzeption, in: Wirtschaftsinformatik, 46. Jg., Heft 1, 2004, S. 39-49.

Becker, Jörg; Köster, Christoph; Sandmann, Dirk (2006): Konsolidierung des Berichtswesens, in: Controlling, 18. Jg., Heft 10, 2006, S. 501-507.

Becker, Jörg; Seidel, Stefan; Janiesch, Christian (2007): Konzeption des Unternehmensreportings - Ein modellgestütztes Vorgehensmodell zur fachkonzeptionellen Spezifikation, in: Oberweis, Andreas; Weinhardt, Christof; Gimpel, Henner; Koschmider, Agnes; Pankratius, Victor; Schnizler, Björn (Hrsg.): eOrganisation: Service-, Prozess-, Market-Engineering: 8. Internationale Tagung Wirtschaftsinformatik, Band 1, Karlsruhe 2007, S. 605-622. 
Becker, Jörg; Seidel, Stefan; Janiesch, Christian (2008): Analyse und Konzeption des Berichtswesens, in: Das Wirtschaftsstudium (Wisu), 37. Jg., Heft 2, 2008, S. 229-233.

Behme, Wolfgang; Ohlendorf, Thomas (1994): Datenbanksysteme, -modelle und Entwurfsmethoden als Grundlage von Controlling-Informationssystemen, in: Biethahn, Jörg; Huch, Burkhard (Hrsg.): Informationssysteme für das Controlling: Konzepte, Methoden und Instrumente zur Gestaltung von Controlling-Informationssystemen, Berlin 1994, S. 117-174.

Beimborn, Daniel; Franke, Jochen; Gomber, Peter; Wagner, Heinz-Theo; Weitzel, Tim (2006): Die Bedeutung des Alignment von IT und Fachressourcen in Finanzprozessen - Eine empirische Untersuchung, in: Wirtschaftsinformatik, 48. Jg., Heft 5, 2006, S. 331-339.

Beimborn, Daniel; Mintert, Stefan; Weitzel, Tim (2002): Web Services und ebXML, in: Wirtschaftsinformatik, 44. Jg., Heft 3, 2002, S. 277-280.

Beimborn, Daniel; Weitzel, Tim (2003): Web Services und Service-oriented IT-Architekturen, in: Das Wirtschaftsstudium (Wisu), 32. Jg., Heft 11, 2003, S. 1360-1364.

Berbner, Rainer; Heckmann, Oliver; Mauthe, Andreas; Steinmetz, Ralf (2005): Eine Dienstgüte unterstützende Webservice-Architektur für flexible Geschäftsprozesse, in: Wirtschaftsinformatik, 47. Jg., Heft 4, 2005, S. 268277.

Besemer, David (2008): Where Structured Data Search Fails, in: Business Intelligence Journal, 13. Jg., Heft 4, 2008, S. 8-16.

Bettag, Urban (2001): Web-Services, in: Informatik-Spektrum, 24. Jg., Heft 10, 2001, S. 302-304.

Beverungen, Daniel; Knackstedt, Ralf; Müller, Oliver (2008): Entwicklung Serviceorientierter Architekturen zur Integration von Produktion und Dienstleistung - Eine Konzeptionsmethode und ihre Anwendung am Beispiel des Recyclings elektronischer Geräte, in: Wirtschaftsinformatik, 50. Jg., Heft 3, 2008, S. 220-234.

Bleek, Wolf-Gideon; Wolf, Henning (2008): Agile Softwareentwicklung: Werte, Konzepte und Methoden, Heidelberg 2008.

Blohm, Hans (1974): Die Gestaltung des betrieblichen Berichtswesens als Problem der Leitungsorganisation, Herne/Berlin 1974.

Blohm, Hans (1980): Berichtssysteme, in: Grochla, Erwin (Hrsg.): Handwörterbuch der Organisation, Stuttgart 1980, S. 315-320.

Blohm, Hans (1982): Berichtswesen, betriebliches, in: Management Enzyklopädie, Band 1, München 1982, S. 866-876. 
Bloom, David (2008): Reinventing Your BI Program, in: Business Intelligence Journal, 13. Jg., Heft 3, 2008, S. 8-19.

Böcking, Hans-Joachim (1998): Zum Verhältnis von Rechnungslegung und Kapitalmarkt: Vom ,financial accounting“ zum „,business reporting“, in: Ballwieser, Wolfgang; Schildbach, Thomas (Hrsg.): Rechnungslegung und Steuern international, zfbf Sonderheft 40, S. 17-53.

Bovee, Matthew; Ettredge, Michael L.; Srivastava, Rajendra P.; Vasarhelyi, Miklos A. (2002): Does the Year 2000 XBRL Taxonomy Accommodate Current Business Financial-Reporting Practice?, in: Journal of Information Systems, 16. Jg., Heft 2, 2002, S. 165-182.

Braunwarth, Kathrin Susanne; Heinrich, Bernd (2008): IT-ServiceManagement - Ein Modell zur Bestimmung der Folgen von Interoperabilitätsstandards auf die Einbindung externer IT-Dienstleister, in: Wirtschaftsinformatik, 50. Jg., Heft 2, 2008, S. 98-110.

Brehm, Nico; Haak, Liane; Gómez, Jorge Marx (2008): Evaluation von Konzepten zur Integration von UDDI-Registries unter Berücksichtigung von Marktaspekten, in: Dinter, Barbara; Winter, Robert; Chamoni, Peter; Gronau, Norbert; Turowski, Klaus (Hrsg.): Synergien durch Integration und Informationslogistik, Bonn 2008, S. 491-508.

Brittenham, Peter (2002): An overview of the Web Services Inspection Language, http://www.ibm.com/developerworks/webservices/library/ws-wsil over, 2002, abgerufen am 23.06.2009.

Brodde, Edgar (2006): SOA erschwert die Qualitätssicherung, in: Computerwoche, 34. Jg., Heft 26, http://www.computerwoche.de/index.cfm?pid= 699\&pk $=578317,2006$, abgerufen am 23.06.2009.

Brown, Frank; Willis, Mike (2003): XBRL: Revolutionizing the Corporate Reporting Supply Chain, in: Financial Executive, o. Jg., Heft 5, 2003, S. 7072.

Buhl, Hans Ulrich (2008): IT-basierte Entscheidungsunterstützung am Beispiel der Subprimekrise: Wunderwaffe oder Teufelszeug?, in: Wirtschaftsinformatik, 50. Jg., Heft 6, 2008, S. 429-433.

Buhl, Hans Ulrich; Heinrich, Bernd; Henneberger, Matthias; Krammer, Alexander (2008): Service Science, in: Wirtschaftsinformatik, 50. Jg., Heft 1, 2008, S. 60-65.

Burghardt, Markus (2004): Web Services - Aspekte von Sicherheit, Transaktionalität, Abrechnung und Workflow, Wiesbaden 2004. 
Busch, Axel; Dangelmaier, Wilhelm (2002): Integriertes Supply Chain Management - ein koordinationsorientierter Überblick, in: Busch, Axel; Dangelmaier, Wilhelm (Hrsg.): Integriertes Supply Chain Management: Theorie und Praxis effektiver unternehmensübergreifender Geschäftsprozesse, Wiesbaden 2002, S. 1-21.

Busse von Colbe, Walther; Laßmann, Gert (1990): Betriebswirtschaftstheorie 3: Investitionstheorie, Berlin/Heidelberg 1990.

Buxmann, Peter (2001): Standardisierung und Netzeffekte, in: Das Wirtschaftsstudium (Wisu), 30. Jg., Heft 4, 2001, S. 544-558.

Buxmann, Peter; Hess, Thomas; Widjaja, Thomas (2007): Serviceorientierte Architekturen, in: Das Wirtschaftsstudium (Wisu), 36. Jg., Heft 10, 2007, S. 1316-1327.

Chamoni, Peter; Linden, Markus (2007): Business Intelligence, in: Das Wirtschaftsstudium (Wisu), 36. Jg., Heft 12, 2007, S. 1588-1592.

Chia, Yew Ming (1995): Decentralization, Management Accounting System (MAS) Information Characteristics and their Interaction Effects on Managerial Performance: A Singapore Study, in: Journal of Business Finance \& Accounting, 22. Jg., Heft 6, 1995, S. 811-830.

Coldewey, Jens (2007): Lohnt sich SOA?, in: Starke, Gernot; Tilkov, Stefan (Hrsg.): SOA-Expertenwissen: Methoden, Konzepte und Praxis serviceorientierter Architekturen, Heidelberg 2007, S. 49-56.

Conrad, Stefan; Hasselbring, Wilhelm; Koschel, Arne; Tritsch, Roland (2006): Enterprise Application Integration: Grundlagen - Konzepte - Entwurfsmuster - Praxisbeispiele, München 2006.

DeFelice, Alexandra (2007): XBRL Grows Up, in: Accounting Technology, 23. Jg., Heft 5, 2007, S. 29-33.

de Hesselle, Bastian; Klüpfel, Sebastian; Bauersachs, Christian (2008): Evaluation von Plattformen für Service-orientierte Architekturen: Der Blick hinter den SOA-Hype, in: is report, 12. Jg., Heft 11, 2008, S. 30-33.

Deppisch, Mario; Oppitz, Michael (2004): Workflow Management, in: Das Wirtschaftsstudium (Wisu), 33. Jg., Heft 4, 2004, S. 886-888.

Devlin, Barry (1997): Data Warehouse: from Architecture to Implementation, Reading, Massachusetts et al. 1997.

Dittmar, Carsten (2004): Knowledge Warehouse: Ein integrativer Ansatz des Organisationsgedächtnisses und die computergestützte Umsetzung auf Basis des Data Warehouse-Konzepts, Wiesbaden 2004. 
Dittmar, Carsten; Schulze, Klaus-Dieter (2006): Der Reifegrad von Business-Intelligence-Lösungen: Seine Stärken kennen lernen, in: BI Spektrum, 1. Jg., Heft 1, 2006, S. 27-31.

Dörner, Dietrich (1989): Die Logik des Misslingens - Strategisches Denken in komplexen Situationen, Reinbek bei Hamburg 1989.

Dorda, Clemens; Steiert, Hans-Peter, Sellentin, Jürgen (2004): Modellbasierter Ansatz zur Anwendungsintegration, in: it - Information Technology, 46. Jg., Heft 4, 2004, S. 200-210.

Dorloff, Frank-Dieter; Leukel, Jörg; Schmitz, Volker (2004a): Datentransformation bei XML-basierten Geschäftsdokumenten, in: Das Wirtschaftsstudium (Wisu), 33. Jg., Heft 1, 2004, S. 87-94.

Dorloff, Frank-Dieter; Leukel, Jörg; Schmitz, Volker (2004b): Konverter für XML-basierte Geschäftsdokumente, in: Das Wirtschaftsstudium (Wisu), 33. Jg., Heft 3, 2004, S. 341-348.

Dueck, Gunter (2006): Inter-Enterprise Services und Innovation, in: Informatik-Spektrum, 29. Jg., Heft 5, 2006, S. 364-368.

Durst, Michael; Daum, Michael (2007): Erfolgsfaktoren serviceorientierter Architekturen, in: HMD - Praxis der Wirtschaftsinformatik, 44. Jg., Heft 253, 2007, S. 18-27.

Eccles, Robert G.; Watson, Liv A.; Willis Mike (2007): Der schnelle Weg zu wichtigen Firmendaten, in: Harvard Business Manager, 29. Jg., Heft 2, 2007, S. 14-15.

Eckerson, Wayne; Sherman, Richard (2008): Strategies for Managing Spreadmarts, in: Business Intelligence Journal, 13. Jg., Heft 1, 2008, S. 2324.

Eckstein, Rainer; Eckstein, Silke (2004): XML und Datenmodellierung: XML-Schema und RDF zur Modellierung von Daten und Metadaten einsetzen, Heidelberg 2004.

Erl, Thomas (2005): Service-Oriented Architecture - Concepts, Technology, and Design, Upper Saddle River, NJ et al. 2005.

Ernst, Hartmut (2002): Grundlagen und Konzepte der Informatik, Braunschweig/Wiesbaden 2002.

Ernst, Thomas; Ember, Georg; Goldhan, Dirk; Kuffner, Marian; Schwarz, Frank (2008): SOA-Projektplanung und Vorgehen, http://soa-know-how.de/ index.php?id=45\&tx_bccatsandauthors[catid] $=20,2008$, abgerufen am 23.06.2009. 
Esch, Dagmar; Ganczarski, Wojciech; Gohr, Volker; Padberg, Jürgen (2008): Serviceorientierte Strukturen zur Unterstützung des integrierten Konzernrechnungswesens, in: ZfCM - Die Zeitschrift für Controlling \& Management, 52. Jg., Sonderheft 2, 2008, S. 92-99.

Eymann, Torsten; Winter, Robert (2008): SOA - Ein neues Paradigma der Gestaltung verteilter Informationssysteme, in: Wirtschaftsinformatik, 50. Jg., Heft 1, 2008, S. 70.

Farewell, Stephanie M. (2006): An Introduction to XBRL through the Use of Research and Technical Assignments, in: Journal of Information Systems, 20. Jg., Heft 1, 2006, S. 161-185.

Federrath, Hannes; Pfitzmann, Andreas (2006): IT-Sicherheit, in: Wind, Martin; Kröger, Detlef (Hrsg.): Handbuch IT in der Verwaltung, Berlin/Heidelberg 2006, S. 273-292.

Felden, Carsten (2006): Ein Format für alle Daten: Business Intelligence und XBRL, in: BI Spektrum, 1. Jg., Heft 3, 2006, S. 32-34.

Fellmann, Michael; Thomas, Oliver (2008): Extensible Markup Language, in: Das Wirtschaftsstudium (Wisu), 37. Jg., Heft 7, 2008, S. 972-976.

Fiege, René; Stelzer, Dirk (2007): Analyse des Beitrages von Axiomatic Design zum Entwurf Serviceorientierter Architekturen, in: Oberweis, Andreas; Weinhardt, Christof; Gimpel, Henner; Koschmider, Agnes; Pankratius, Victor; Schnizler, Björn (Hrsg.): eOrganisation: Service-, Prozess-, MarketEngineering: 8. Internationale Tagung Wirtschaftsinformatik, Band 2, Karlsruhe 2007, S. 909-926.

Fink, Andreas; Schneidereit, Gabriele; Voß, Stefan (2005): Grundlagen der Wirtschaftsinformatik, Heidelberg 2005.

Foegen, Malte; Battenfeld, Jörg (2001): Die Rolle der Architektur in der Anwendungsentwicklung, in: Informatik-Spektrum, 24. Jg., Heft 5, 2001, S. 290-301.

Frosch-Wilke, Dirk (2003): Data Warehouse, OLAP und Data Mining, in: Datenschutz und Datensicherheit (DuD), 27. Jg., Heft 10, 2003, S. 597-604.

Frotscher, Thilo (2007): Der Webservices-Technologiestack, in: Starke, Gernot; Tilkov, Stefan (Hrsg.): SOA-Expertenwissen: Methoden, Konzepte und Praxis serviceorientierter Architekturen, Heidelberg 2007, S. 489-506.

Gabaix, Xavier; Laibson, David; Moloche, Guillermo; Weinberg, Stephen (2006): Costly Information Acquisition: Experimental Analysis of a Boundedly Rational Model, in: The American Economic Review, 96. Jg., Heft 4, 2006, S. 1043-1068. 
Gabriel, Roland; Beier, Dirk (2003): Informationsmanagement in Organisationen, Stuttgart 2003.

Gabriel, Roland; Gluchowski, Peter; Pastwa, Alexander (2006): Extensible Business Reporting Language (XBRL) für das Rechnungswesen, in: Das Wirtschaftsstudium (Wisu), 35. Jg., Heft 7, 2006, S. 938-944.

Gabriel, Roland; Hoppe, Tobias; Pastwa, Alexander (2008): Intelligente Wohnungsmarktanalyse auf Basis einer Business Intelligence-Anwendung Anforderungen, Modellierung und prototypische Realisierung, in: Dinter, Barbara; Winter, Robert; Chamoni, Peter; Gronau, Norbert; Turowski, Klaus (Hrsg.): Synergien durch Integration und Informationslogistik, Bonn 2008, S. 287-298.

Gabriel, Roland; Röhrs, Heinz-Peter (1995): Datenbanksysteme: Konzeptionelle Datenmodellierung und Datenbankarchitekturen, Berlin et al. 1995.

Gabriel, Roland; Röhrs, Heinz-Peter (2003): Gestaltung und Einsatz von Datenbanksystemen: Data Base Engineering und Datenbankarchitekturen, Berlin et al. 2003.

Garbellotto, Gianluca (2006a): Exposing Enterprise Data: XBRL GL, Web Services, and Google, Part 1, in: Strategic Finance, 87. Jg., Heft 8, 2006, S. 59-61.

Garbellotto, Gianluca (2006b): Exposing Enterprise Data: XBRL GL, Web Services, and Google, Part 2, in: Strategic Finance, 87. Jg., Heft 9, 2006, S. 59-61.

Gassen, Joachim (2000): Datenbankgestützte Rechnungslegungspublizität: Ein Beitrag zur Evolution der Rechnungslegung, Frankfurt am Main et al. 2000.

Gehra, Berhard; Hess, Thomas (2004): XBRL - Ein Kommunikationsstandard für das Financial Reporting, in: ZfCM - Die Zeitschrift für Controlling \& Management, 48. Jg., Heft 6, 2004, S. 403-405.

Gehring, Hermann; Gadatsch, Andreas (1999): Ein Rahmenkonzept für die Modellierung von Geschäftsprozessen und Workflows, Fachbereichsbericht Nr. 274 des Fachbereichs Wirtschaftswissenschaft, FernUniversität Hagen, ftp://ftp.fernuni-hagen.de/pub/fachb/wiwi/winf/forschng/publi/hg_p15.pdf, 1999, abgerufen am 23.06.2009.

Gericke, Anke; Stutz, Matthias (2006): Internetressourcen zu IT/Business Alignment, in: Wirtschaftsinformatik, 48. Jg., Heft 5, 2006, S. 362-367.

Gimnich, Rainer (2007): SOA-Migration - Vorgehen und Projekterfahrungen, in: HMD - Praxis der Wirtschaftsinformatik, 44. Jg., Heft 257, 2007, S. 6779. 
Gleich, Ronald; Sasse, Alexander; Gräf, Jens; Kogler, Sabine (2002): Corporate Reporting: Empirische Erkenntnisse und Impulse zur Performancesteigerung, in: Controlling, 14. Jg., Heft 6, 2002, S. 337-345.

Glöckle, Herbert (2007): IT-Integration und Migration - Konzepte und Vorgehensweisen, in: HMD - Praxis der Wirtschaftsinformatik, 44. Jg., Heft 257, 2007, S. 7-19.

Gluchowski, Peter (1998): Werkzeuge zur Implementierung des betrieblichen Berichtswesens, in: Das Wirtschaftsstudium (Wisu), 27. Jg., Heft 10, 1998, S. 1174-1188.

Gluchowski, Peter (2001): Business Intelligence: Konzepte, Technologien und Einsatzbereiche, in: HMD - Praxis der Wirtschaftsinformatik, 38. Jg., Heft 222, 2001, S. 5-15.

Gluchowski, Peter (2006): Techniken und Werkzeuge zum Aufbau betrieblicher Berichtssysteme, in: Chamoni, Peter; Gluchowski, Peter (Hrsg.): Analytische Informationssysteme: Business Intelligence-Technologien und Anwendungen, Berlin et al. 2006, S. 207-226.

Gluchowski, Peter; Gabriel, Roland; Chamoni, Peter (1997): Management Support Systeme - Computergestützte Informationssysteme für Führungskräfte und Entscheidungsträger, Berlin/Heidelberg 1997.

Gluchowski, Peter; Gabriel, Roland; Dittmar, Carsten (2007): Management Support Systeme und Business Intelligence - Computergestützte Informationssysteme für Fach- und Führungskräfte, Berlin/Heidelberg 2007.

Gluchowski, Peter; Kemper, Hans-Georg (2006): Quo Vadis Business Intelligence?, in: BI Spektrum, 1. Jg., Heft 1, 2006, S. 12-19.

Gluchowski, Peter; Pastwa, Alexander (2006): Reporting-Standard vor dem Durchbruch - Details über Grundlagen, Bausteine, Erweiterungen und Potenziale der erhofften „Lingua Franca“ des Finanz-Berichtswesens: XBRL, in: is report, 10. Jg., Heft $1 \frac{1}{2}$, 2006, S. 66-69.

Göpfert, Ingrid (2002): Berichtswesen, in: Küpper, Hans-Ulrich; Wagenhofer, Alfred (Hrsg.): Handwörterbuch Unternehmensrechnung und Controlling, Stuttgart 2002, S. 143-156.

Göricke, Nico; Kirchhof, Oliver (2006): Optimierung der Prozesse der Konzernberichterstattung, in: Information Management \& Consulting (IM), 21. Jg., Heft 3, 2006, S. 54-58.

Grabowski, Götz (2008): Der letzte Excel-Cowboy, in: is report, 12. Jg., Heft 10,2008 , S. 22-23.

Grimm, Sebastian (2005): Prozessportale: Schnittstelle zwischen Anwendern und Unternehmen, in: IT Management, o. Jg., Heft 10, 2005, S. 16-22. 
Gronau, Norbert; Stein, Moreen; Eggert, Sandy; Lämmer, Anne (2008): Integration öffentlicher Anwendungslandschaften, in: Dinter, Barbara; Winter, Robert; Chamoni, Peter; Gronau, Norbert; Turowski, Klaus (Hrsg.): Synergien durch Integration und Informationslogistik, Bonn 2008, S. 425-436.

Grothe, Martin (2005): Business Intelligence in Telco-Unternehmen, in: ZfCM - Die Zeitschrift für Controlling \& Management, 49. Jg., Sonderheft 2, 2005, S. 136-144.

Grothe, Martin; Gentsch, Peter (2000): Business Intelligence - Aus Informationen Wettbewerbsvorteile gewinnen, München 2000.

Gruppe Deutsche Börse (2009): Gruppe Deutsche Börse, http://deutscheboerse.com/dbag/dispatch/de/kir/gdb_navigation/home, 2009, abgerufen am 23.06.2009.

Hahn, Oliver (2003): Data Warehousing und Data Mining in der Praxis, in: Datenschutz und Datensicherheit (DuD), 27. Jg., Heft 10, 2003, S. 605-608.

Hammerschall, Ulrike (2005): Verteilte Systeme und Anwendungen - Architekturkonzepte, Standards und Middleware-Technologien, München 2005.

Hanhart, Daniel; Legner, Christine; Österle, Hubert (2008): Eine Serviceorientierte Architektur (SOA) für die Integration von RFID-, Sensor- und Lokalisierungsdaten im Facility Management, in: Dinter, Barbara; Winter, Robert; Chamoni, Peter; Gronau, Norbert; Turowski, Klaus (Hrsg.): Synergien durch Integration und Informationslogistik, Bonn 2008, S. 475-490.

Hannon, Neal (2004): XBRL and Metcalfe's Rule of Technological Change, in: Strategic Finance, 85. Jg., Heft 1, 2004, S. 57-58.

Hannon, Neal (2005): XBRL Fundamentals, in: Strategic Finance, 86. Jg., Heft 4,2005 , S. 57-58.

Hannon, Neal (2006): In Search of ROI for XBRL, in: Strategic Finance, 87. Jg., Heft 3, 2006, S. 59-60.

Hansen, Hans Robert; Neumann, Gustaf (2005): Wirtschaftsinformatik I: Grundlagen der betrieblichen Informationsverarbeitung, Stuttgart 2005.

Härder, Theo; Rahm, Erhard (2001): Datenbanksysteme: Konzepte und Techniken der Implementierung, Berlin/Heidelberg 2001.

Heinrich, Lutz J. (2002): Informationsmanagement: Planung, Überwachung und Steuerung der Informationsinfrastruktur, München 2002.

Heinrich, Bernd; Klier, Mathias; Bewernik, Marc-Andre (2006): Unternehmensweite Anwendungsintegration - Zentrale Anreizsetzung zur Realisierung von Netzwerkeffekten bei dezentralen Entscheidungsstrukturen, in: Wirtschaftsinformatik, 48. Jg., Heft 3, 2006, S. 158-168. 
Hellriegel, Don; Jackson Susan E.; Slocum, John W. (1999): Management, Cincinnati, Ohio 1999.

Helmerich, Jürgen; Hümpfner, Klaus-Volker; Scherer, Jürgen (2004): XML - von der Dokumentstrukturierung zur Datenspeicherung, in: Das Wirtschaftsstudium (Wisu), 33. Jg., Heft 5, 2004, S. 623-625.

Herden, Sebastian; Gómez, Jorge Marx; Rautenstrauch, Claus; Zwanziger, André (2006): Software-Architekturen für das E-Business - EnterpriseApplication-Integration mit verteilten Systemen, Berlin/Heidelberg 2006.

Herrmann, Clemens; Müller, Stefan-Alexander (2008): Business Intelligence Services bei T-Mobile Deutschland: Service Level Agreements und servicebezogenes Datenqualitätsmanagement zur kundengerechten Leistungserbringung, in: Dinter, Barbara; Winter, Robert; Chamoni, Peter; Gronau, Norbert; Turowski, Klaus (Hrsg.): Synergien durch Integration und Informationslogistik, Bonn 2008, S. 229-248.

Heß, Helge (2005): Von der Unternehmensstrategie zur Prozess-Performance Was kommt nach Business Intelligence?, in: Scheer, August-Wilhelm; Jost, Wolfram; Heß, Helge; Kronz, Andreas (Hrsg.): Corporate Performance Management: ARIS in der Praxis, Berlin/Heidelberg 2005, S. 7-30.

Hess, Andreas; Humm, Bernhard; Voß, Markus (2006): Regeln für serviceorientierte Architekturen hoher Qualität, in: Informatik-Spektrum, 29. Jg., Heft 6, 2006, S. 395-411.

Hettler, Dora; Preuss, Peter; Niedereichholz, Joachim (2003): Vergleich ausgewählter Ansätze zur semantischen Modellierung von Data-WarehouseSystemen, in: HMD - Praxis der Wirtschaftsinformatik, 40. Jg., Heft 231, 2003, S. 97-107.

Heuser, Lutz; Lacher, Stefan; Perlmann, Simone (2007): Flexible Prozessgestaltung als Basis innovativer Geschäftsmodelle: Von der ServiceOrientierten Architektur zur Vision des Business Webs, in: Oberweis, Andreas; Weinhardt, Christof; Gimpel, Henner; Koschmider, Agnes; Pankratius, Victor; Schnizler, Björn (Hrsg.): eOrganisation: Service-, Prozess-, Market-Engineering: 8. Internationale Tagung Wirtschaftsinformatik, Band 1, Karlsruhe 2007, S. 19-28.

Heutschi, Roger (2007): Serviceorientierte Architektur - Architekturprinzipien und Umsetzung in die Praxis, Berlin 2007.

Heutschi, Roger; Legner, Christine; Österle, Hubert (2006): Serviceorientierte Architekturen: Vom Konzept zum Einsatz in der Praxis, in: Schelp, Joachim; Winter, Robert; Frank, Ulrich; Rieger, Bodo; Turowski, Klaus (Hrsg.): Integration, Informationslogistik und Architektur, Bonn 2006, S. 361-382. 
Hinkelmann, Knut; Thönssen, Barbara; Probst, Fabian (2005): Referenzmodellierung für E-Government-Services, in: Wirtschaftsinformatik, 47. Jg., Heft 5, 2005, S. 356-366.

Hirsch, Bernhard; Paefgen, Anne; Schaier, Sven (2008): Gestaltung von Monatsberichten in deutschen Großunternehmen, in: ZfCM - Die Zeitschrift für Controlling \& Management, 52. Jg., Heft 5, 2008, S. 326-332.

Hirsch, Bernhard; Sorg, Mascha (2008): Controller und Investor Relations: Konzeptionelle und empirische Untersuchung der Schnittstellen, in: Finanz Betrieb, 8. Jg., Heft 7-8, 2008, S. 428-434.

Hodge, Frank D.; Kennedy, Jane J.; Maines, Laureen A. (2004): Does Search-Facilitating Technology Improve the Transparency of Financial Reporting?, in: The Accouting Review, 79. Jg., Heft 3, 2004, S. 687-703.

Hoffmann, Werner; Kusterer, Frank (1997): Handels-Controlling auf Basis eines Datawarehouse und OLAP, in: Controlling, 9. Jg., Heft 1, 1997, S. 4653.

Hofmann, Oliver (2003): Web-Services in serviceorientierten ITArchitekturkonzepten, in: HMD - Praxis der Wirtschaftsinformatik, 40. Jg., Heft 234, 2003, S. 27-33.

Holten, Roland (2003): Integration von Informationssystemen, in: Wirtschaftsinformatik, 45. Jg., Heft 1, 2003, S. 41-52.

Holtschke, Bernhard; Heier, Hauke; Hummel, Thomas (2009): Quo vadis CIO?, Berlin/Heidelberg 2009.

Hoppe, Tobias; Pastwa, Alexander (2007): Dem Täter auf der Spur, in: BI Spektrum, 2. Jg., TDWI-Sonderheft, 2007, S. 39-43.

Horváth, Péter (2006): Controlling, München 2006.

Höß, Oliver; Weisbecker, Anette; Specht, Thomas; Drawehn, Jens (2007): Migration zu serviceorientierten Architekturen - top-down oder bottom-up?, in: HMD - Praxis der Wirtschaftsinformatik, 44. Jg., Heft 257, 2007, S. 3946.

Humm, Bernhard; Juwig, Oliver (2006): Eine Normalform für Services, http://www.fbi.h-da.de/fileadmin/personal/b.humm/Publikationen/Humm Juwig_-_Normalform_Services_Software_Engineering_2006_.pdf, 2006, abgerufen am 23.06.2009.

Huth, Stefan; Wieland, Thomas (2008): Geschäftsprozessmodellierung mittels Software-Services auf Basis der EPK, http://www.hs-coburg.de/ fileadmin/FB_EINF/staff/wieland/papers/services-EPK.pdf, Coburg 2008, abgerufen am 23.06.2009. 
IASB (2005): International Financial Reporting Standards, General Purpose Financial Reporting for Profit-Oriented Entities, Incorporating Additional Requirements for Banks and Similar Financial Institutions (IFRS-GP), 15.05.2005, http://xbrl.iasb.org/int/fr/ifrs/gp/2005-05-15/summary_page.htm, 2005, abgerufen am 23.06.2009.

Issing, Frank (2003): Die Softwarestrategie für Web-Services und Unternehmensinfrastrukturen der Firma Sun Microsystems, in: HMD - Praxis der Wirtschaftsinformatik, 40. Jg., Heft 234, 2003, S. 17-26.

Jarke, Matthias; Lenzerini, Maurizio; Vassiliou, Yannis; Vassiliadis, Panos (2000): Fundamentals of Data Warehouses, Berlin et al. 2000.

Jenner, Thomas (2000): Zur Messung des Unternehmenserfolges, in: Das Wirtschaftsstudium (Wisu), 29. Jg., Heft 3, 2000, S. 326-331.

Josuttis, Nicolai (2007): SOA und Performance, in: Starke, Gernot; Tilkov, Stefan (Hrsg.): SOA-Expertenwissen: Methoden, Konzepte und Praxis serviceorientierter Architekturen, Heidelberg 2007, S. 381-388.

Josuttis, Nicolai (2008): SOA in der Praxis: System-Design für verteilte Geschäftsprozesse, Heidelberg 2008.

Kaczmarek, Tomasz; Wecel, Krzysztof (2008): Hype over Service Oriented Architecture Continues, in: Wirtschaftsinformatik, 50. Jg., Heft 1, 2008, S. 52-58.

Kaib, Michael (2002): Enterprise Application Integration: Grundlagen, Integrationsprodukte, Anwendungsbeispiele, Wiesbaden 2002.

Karch, Steffen; Heilig, Loren; Bernhardt, Christian; Hardt, Andreas; Heidfeld, Frank; Pfennig, Roland (2004): SAP NetWeaver, Bonn 2004.

Kazakos, Wassilios; Schmidt, Andreas; Tomczyk, Peter (2002): Datenbanken und XML: Konzepte, Anwendungen, Systeme, Berlin/Heidelberg/ New York 2002.

Keller, Wolfgang (2002): Enterprise Application Integration: Erfahrungen aus der Praxis, Heidelberg 2002.

Keller, Wolfgang (2007): Regeln und Heuristiken für gutes Schnittstellendesign, in: Starke, Gernot; Tilkov, Stefan (Hrsg.): SOA-Expertenwissen: Methoden, Konzepte und Praxis serviceorientierter Architekturen, Heidelberg 2007, S. 357-380.

Kemper, Hans-Georg; Mehanna, Walid; Unger, Carsten (2004): Business Intelligence - Grundlagen und praktische Anwendungen: Eine Einführung in die IT-basierte Managementunterstützung, Wiesbaden 2004. 
Kink, Natalie (2008): Anwendungssystemintegration und Unternehmensgrenzen, in: ZfCM - Die Zeitschrift für Controlling \& Management, 52. Jg., Heft 6, 2008, S. 359-360.

Kink, Natalie; Höhne, Elisabeth; Hess, Thomas (2008): Wirkungen von Management Support Systemen (MSS) auf die Steuerung von Unternehmen, in: ZfCM - Die Zeitschrift für Controlling \& Management, 52. Jg., Sonderheft 2, 2008, S. 5-14.

Kleinschmidt, Peter; Rank, Christian (2002): Relationale Datenbanksysteme: Eine praktische Einführung, Berlin/Heidelberg 2002.

Klempt, Philipp; Werners, Brigitte (2009): Tool-basierte Evaluation und Steuerung der unternehmensweiten Informationssicherheit, in: Meyer, JörnAxel (Hrsg.): Management-Instrumente in kleinen und mittleren Unternehmen, Jahrbuch der KMU-Forschung und -Praxis 2009, Köln 2009, S. 307329.

Klesse, Mario; Wortmann, Felix; Schelp, Joachim (2005): Erfolgsfaktoren der Applikationsintegration, in: Wirtschaftsinformatik, 47. Jg., Heft 4, 2005, S. 259-267.

Klettke, Meike; Meyer, Holger (2003): XML \& Datenbanken: Konzepte, Sprachen und Systeme, Heidelberg 2003.

Klose, Karsten; Knackstedt, Ralf (2007): Serviceidentifikation für die Produktionsplanung eines mittelständischen Auftragsfertigers, in: HMD - Praxis der Wirtschaftsinformatik, 44. Jg., Heft 253, 2007, S. 47-56.

Koch, Rembert (1994): Betriebliches Berichtswesen als Informations- und Steuerungsinstrument, Europäische Hochschulschriften, Reihe 5: Volks- und Betriebswirtschaft, Band 1483, Frankfurt am Main 1994.

Kossmann, Donald; Leymann, Frank (2004): Web Services, in: InformatikSpektrum, 27. Jg., Heft 2, 2004, S. 117-128.

Köthner, Dietmar (2006): Reporting: 50 Prozent des Aufwandes einsparen, in: is report, 10. Jg., Heft 11, 2006, S. 6.

Köthner, Dietmar (2008): Befragung: Excel alleine reicht für die Planung nicht aus, in: is report, 12. Jg., Heft 10, 2008, S. 26.

Krafzig, Dirk; Banke, Karl; Slama, Dirk (2007): Enterprise SOA: Best Practices für Serviceorientierte Architekturen - Einführung, Umsetzung, Praxis, Frechen 2007.

Kranich, Pascal; Schmitz, Hans (2003): Die Extensible Business Reporting Language - Standard, Taxonomien und Entwicklungsperspektiven, in: Wirtschaftsinformatik, 45. Jg., Heft 1, 2003, S. 77-80. 
Krause, Herbert; Schmitz, Uwe (2006): Entwicklung einer Methode für eine werttreiberbezogene Informationsbedarfsermittlung, in: Controlling, 18. Jg., Heft 7, 2006, S. 351-361.

Krcmar, Helmut (2003): Informationsmanagement, Berlin/Heidelberg 2003.

Kronz, Andreas (2005): Management von Prozesskennzahlen im Rahmen der ARIS-Methodik, in: Scheer, August-Wilhelm; Jost, Wolfram; Heß, Helge; Kronz, Andreas (Hrsg.): Corporate Performance Management: ARIS in der Praxis, Berlin/Heidelberg 2005, S. 31-44.

Kruczynski, Klaus (2006): DW 2.0 - das neue Konzept für Data Warehousing, in: is report, 10. Jg., Heft 12, 2006, S. 22-25.

Kruppke, Helmut; Bauer, Tino (2005): Keine Business Intelligence ohne Process Intelligence, in: Scheer, August-Wilhelm; Jost, Wolfram; Heß, Helge; Kronz, Andreas (Hrsg.): Corporate Performance Management: ARIS in der Praxis, Berlin/Heidelberg 2005, S. 77-98.

Kubli, Rolf; Burkard, Michael; Rantakallio, Elina; Kreczy, Annette (2008): SOA Implementierung in der Luftfahrtindustrie am Beispiel der Lufthansa, in: Dinter, Barbara; Winter, Robert; Chamoni, Peter; Gronau, Norbert; Turowski, Klaus (Hrsg.): Synergien durch Integration und Informationslogistik, Bonn 2008, S. 543-558.

Kuhn, Thomas S. (1976): Die Struktur wissenschaftlicher Revolutionen, Frankfurt am Main 1976.

Kuhn, Alfred (1990): Unternehmensführung, München 1990.

Künkele, Julia; Schäffer, Utz (2007): Zur erfolgreichen Gestaltung der Budgetkontrolle - Ergebnisse einer empirischen Untersuchung, in: Die Betriebswirtschaft (DBW), 67. Jg., Heft 1, 2007, S. 75-92.

Küpper, Hans-Ulrich (1997): Controlling, Stuttgart 1997.

Kurz, Andreas (1999): Data Warehousing: Enabling Technology, Bonn 1999.

Küster, Marc W. (2003): Web-Services - Versprechen und Realität, in: HMD - Praxis der Wirtschaftsinformatik, 40. Jg., Heft 234, 2003, S. 5-15.

Laartz, Jürgen (2008): SOA revolutioniert das Management, in: Wirtschaftsinformatik, 50. Jg., Heft 1, 2008, S. 72-73.

Lankes, Josef; Stapel, Stephan; Herwig, Volker; Ernst, Alexander M. (2008): Die nächste Generation von Unternehmensdatenmodellen? Management von Geschäftsobjekten - Informationsmodellierung auf Unternehmensebene, in: is report, 12. Jg., Heft 11, 2008, S. 12-18.

Laudon, Kenneth C.; Laudon, Jane P.; Schoder, Detlef (2006): Wirtschaftsinformatik - Eine Einführung, München 2006. 
Lauer, Daniel; Juwig, Oliver (2007): Qualitätssicherung in einer serviceorientierten Architektur, in: Starke, Gernot; Tilkov, Stefan (Hrsg.): SOAExpertenwissen: Methoden, Konzepte und Praxis serviceorientierter Architekturen, Heidelberg 2007, S. 263-284.

Lehmann Peter; Ellerau, Peter (1997): Implementierung eines Data Warehouse für die Verpackungsindustrie, in: HMD - Praxis der Wirtschaftsinformatik, 34. Jg., Heft 195, 1997, S. 76-93.

Lehner, Franz (2000): Organisational Memory: Konzepte und Systeme für das organisatorische Lernen und das Wissensmanagement, München/Wien 2000.

Leitl, Michael (2006): Was sind Stakeholder?, in: Harvard Businessmanager, 28. Jg., Heft 7, 2006, S. 10.

Leser, Ulf; Naumann, Felix (2007): Informationsintegration - Architekturen und Methoden zur Integration verteilter und heterogener Datenquellen, Heidelberg 2007.

Leßweng, Hans-Peter (2003a): Business Intelligence Tools: Plädoyer für die Integration des Prozesses Berichtsdiskussion, in: Uhr, Wolfgang; Esswein, Werner; Schoop, Eric (Hrsg.): Wirtschaftsinformatik 2003: Medien - Märkte - Mobilität, Band 2, Heidelberg 2003, S. 333-352.

Leßweng, Hans-Peter (2003b): Einsatz der Internet-Technologie im betrieblichen Berichtswesen, Köln 2003.

Leßweng, Hans-Peter (2004): Einsatz von Business Intelligence Tools (BIT) im betrieblichen Berichtswesen, in: Controlling, 16. Jg., Heft 1, 2004, S. 4149.

Lev, Baruch; Zarowin, Paul (1999): The Boundaries of Financial Reporting and How to Extend Them, in: Journal of Accounting Research, 37. Jg., Heft 2, 1999, S. 353-385.

Leyking, Katrina; Ziemann, Jörg; Loos, Peter (2006): Geschäftsprozessmodelle und serviceorientierte Architekturen (SOA), in: Das Wirtschaftsstudium (Wisu), 35. Jg., Heft 8-9, 2006, S. 1037-1040.

Liebetruth, Thomas; Otto, Andreas (2006): Ein formales Modell zur Auswahl von Kennzahlen, in: Controlling, 18. Jg., Heft 1, 2006, S. 13-23.

Liebhart, Daniel (2007): SOA goes real - Service-orientierte Architekturen erfolgreich planen und einführen, München/Wien 2007.

Liebowitz, Jay (2006): Strategic Intelligence: Business Intelligence, Competitive Intelligence, and Knowledge Management, Boca Raton, Florida 2006. 
Ließmann, Harald; Kaufmann, Thomas; Schmitzer, Benno (1999): Bussysteme als Schlüssel zur betriebswirtschaftlich-semantischen Kopplung von Anwendungssystemen, in: Wirtschaftsinformatik, 41. Jg., Heft 1, 1999, S. 1219.

Luhmann, Till; Meister, Jürgen; Wulff, Christian (2007): Serviceorientierte Produktplattform für das Energiemanagementsystem der Zukunft, in: Wirtschaftsinformatik, 49. Jg., Heft 5, 2007, S. 343-351.

Lymer, Andy (1999): The Internet and the future of corporate reporting in Europe, in: The European Accounting Review, 8. Jg., Heft 2, 1999, S. 289301.

Mahlberg, Michael (2007): SOA "von unten«, in: Starke, Gernot; Tilkov, Stefan (Hrsg.): SOA-Expertenwissen: Methoden, Konzepte und Praxis serviceorientierter Architekturen, Heidelberg 2007, S. 169-186.

Mantel, Stephan; Schissler, Martin (2002): Application Integration, in: Wirtschaftsinformatik, 44. Jg., Heft 2, 2002, S. 171-174.

Martens, Wim; Neven, Frank; Schwentick, Thomas; Bex, Geert J. (2006): Expressiveness and Complexity of XML Schema, in: ACM Transactions on Database Systems, 31. Jg., Heft 3, 2006, S. 770-813.

Mas y Parareda, Benedikt; Pizka, Markus (2007): Web-basierte und andere junge Legacy-Systeme, in: Information Management \& Consulting, 22. Jg., Heft 2, 2007, S. 60-68.

Mattern, Thomas (2003): Web-Services als Basis neuer betriebswirtschaftlicher Konzepte, in: HMD - Praxis der Wirtschaftsinformatik, 40. Jg., Heft 234,2003, S. 34-41.

Meall, Lesley (2007): Taming the beast: Has the eXtensible Business Reporting Language finally come of age?, in: accountancymagazine.com, o. Jg., Heft 7, 2007, S. 73-74.

Melzer, Ingo; Dostal, Wolfgang; Jeckle, Mario; Zengler, Barbara (2007): Service-orientierte Architekturen mit Web Services: Konzepte - Standards Praxis, München 2007.

Mertens, Peter (1999): Integration interner, externer, qualitativer und quantitativer Daten auf dem Weg zum Aktiven MIS, in: Wirtschaftsinformatik, 41. Jg., Heft 5, 1999, S. 405-415.

Mertens, Peter; Billmeyer, Andreas; Bradl, Peter (2003): Informationsverarbeitung in der strategischen Unternehmensplanung, in: Das Wirtschaftsstudium (Wisu), 32. Jg., Heft 6, 2003, S. 795-803.

Mertens, Peter; Griese, Joachim (2002): Integrierte Informationsverarbeitung 2: Planungs- und Kontrollsysteme in der Industrie, Wiesbaden 2002. 
Meyer-Pries, Lars; Gröner, Susanne (2002): Web-Publizität und Datenaustausch mit XBRL, in: Finanzbetrieb, 4. Jg., Heft 1, 2002, S. 44-53.

Mintert, Stefan (2005): Implementierung von Webservices: REST vs. SOAP?, in: Wirtschaftsinformatik, 47. Jg., Heft 1, 2005, S. 63-65.

Modjo Kamneng, Martin L.; Brandt, Volker; Steckel, Thilo; Nüßer, Willi (2007): Prozessintegration mobiler Landmaschinen mittels automatisch erzeugter BPEL-Prozesse, in: Wirtschaftsinformatik, 49. Jg., Heft 4, 2007, S. 289-294.

Mucksch, Harry; Behme, Wolfgang (2001): Anwendungsgebiete einer Data Warehouse-gestützten Informationsverarbeitung, in: Behme, Wolfgang; Mucksch, Harry (Hrsg.): Data Warehouse-gestützte Anwendungen: Theorie und Praxiserfahrungen in verschiedenen Branchen, Wiesbaden 2001, S. 3-32.

Müller, Andreas M.; Hess, Thomas (2006): Integration von Anwendungssystemen: eine netzeffekttheoretische Analyse des Nutzens, in: ZfCM - Die Zeitschrift für Controlling \& Management, 50. Jg., Heft 2, 2006, S. 108-116.

Müller, Felix R. (2007a): Anwendungsarchitektur-Beurteilung unter Berücksichtigung zu erwartender Flexibilität, in: Oberweis, Andreas; Weinhardt, Christof; Gimpel, Henner; Koschmider, Agnes; Pankratius, Victor; Schnizler, Björn (Hrsg.): eOrganisation: Service-, Prozess-, Market-Engineering: 8. Internationale Tagung Wirtschaftsinformatik, Band 2, Karlsruhe 2007, S. 89106.

Müller, Sascha; Kuhn, Walter; Meiler, Christian; Petrov, Ilia; Jablonski, Stefan (2006): Integratives IT-Architekturmanagement, in: Reussner, Ralf; Hasselbring, Wilhelm (Hrsg.): Handbuch der Software-Architektur, Heidelberg 2006, S. 187-210.

Müller, Rudolf; Klatt, Michael; Pfitzmayer, Karl-Heinz (2000): Das Spannungsfeld Konzernreporting, in: Controller Magazin, 31. Jg., Heft 4, 2000, S. 354-360.

Müller, Thomas (2007b): Der Weg zum guten Service, in: Starke, Gernot; Tilkov, Stefan (Hrsg.): SOA-Expertenwissen: Methoden, Konzepte und Praxis serviceorientierter Architekturen, Heidelberg 2007, S. 141-159.

Nastansky, Ludwig; Huth, Carsten (2006): Workflow-Management - Systemstatus und Entwicklungsströmungen, in: Das Wirtschaftsstudium (Wisu), 35. Jg., Heft 4, 2006, S. 528-541.

Navrade, Frank (2006): Strategische Planung: Zusammenhänge sehen und steuern, in: BI Spektrum, 1. Jg., Heft 2, 2006, S. 12-15.

Newcomer, Eric; Lomow, Greg (2005): Understanding SOA with Web Services, Upper Saddle River, NJ et al. 2005. 
Niemann, Heiko; Hasselbring, Wilhelm; Wendt, Thomas; Winter, Alfred; Meierhofer, Matthias (2002): Kopplungsstrategien für Anwendungssysteme im Krankenhaus, in: Wirtschaftsinformatik, 44. Jg., Heft 5, 2002, S. 425-434.

Nüttgens, Markus; Dirik, Iskender (2008): Geschäftsmodelle für dienstebasierte Informationssysteme - Ein strategischer Ansatz zur Vermarktung von Webservices, in: Wirtschaftsinformatik, 50. Jg., Heft 1, 2008, S. 31-38.

Nüttgens, Markus; Rump, Frank J. (2002): Syntax und Semantik Ereignisgesteuerter Prozessketten (EPK), in: Desel, Jörg; Weske, Mathias (Hrsg.): Prozessorientierte Methoden und Werkzeuge für die Entwicklung von Informationssystemen (Promise 2002), Hasso-Plattner-Institut für Softwaresystemtechnik an der Universität Potsdam, 9.-11. Oktober 2002, Bonn 2002, S. 64-77.

Nutz, Andreas; Strauß, Markus (2002): eXtensible Business Reporting Language (XBRL) - Konzept und praktischer Einsatz, in: Wirtschaftsinformatik, 44. Jg., Heft 5, 2002, S. 447-457.

OASIS (2008): OASIS, http://www.oasis-open.org, 2008, abgerufen am 23.06.2009.

Object Management Group (2006): Business Process Modeling Notation (BPMN) Specification, http://www.bpmn.org/Documents/OMG\%20Final\% 20Adopted\%20BPMN\%201-0\%20Spec\%2006-02-01.pdf, 2006, abgerufen am 23.06.2009.

Oehler, Karsten (2006): Corporate Performance Management mit Business Intelligence Werkzeugen, München/Wien 2006.

Oestereich, Bernd (2007): SOA - Mythos und Wahrheit, in: Starke, Gernot; Tilkov, Stefan (Hrsg.): SOA-Expertenwissen: Methoden, Konzepte und Praxis serviceorientierter Architekturen, Heidelberg 2007, S. 633-650.

Oestereich, Bernd; Weiss, Christian (2008): APM - Agiles Projektmanagement: Erfolgreiches Timeboxing für IT-Projekte, Heidelberg 2008.

Offermann, Philipp (2008): SOAM - Eine Methode zur Konzeption betrieblicher Software mit einer Serviceorientierten Architektur, in: Wirtschaftsinformatik, 50. Jg., Heft 6, 2008, S. 461-471.

Pandrangi, Srinivas (2003): A Technical Overview of XBRL, http://xml.syscon.com/node/40706, 2003, abgerufen am 23.06.2009.

Pellens, Bernhard; Fülbier, Rolf U.; Gassen, Joachim (2006): Internationale Rechnungslegung, Stuttgart 2006.

Pernul, Günther; Unland, Rainer (2003): Datenbanken im Unternehmen: Analyse, Modellbildung und Einsatz, München/Wien 2003. 
Petersohn, Helge (2004): Data-Mining-Anwendungsarchitektur, in: Wirtschaftsinformatik, 46. Jg., Heft 1, 2004, S. 15-21.

Petersohn, Helge (2005): Data Mining: Verfahren, Prozesse, Anwendungsarchitektur, München 2005.

Poluha, Rolf G. (2007): Anwendung des SCOR-Modells zur Analyse der Supply Chain - Explorative empirische Untersuchung von Unternehmen aus Europa, Nordamerika und Asien, Köln 2007.

Pomberger, Gustav; Blaschek, Günther (1993): Grundlagen des Software Engineering: Prototyping und objektorientierte Software-Entwicklung, München/Wien 1993.

Pomberger, Gustav; Pree, Wolfgang (2004): Software Engineering: Architektur-Design und Prozessorientierung, München et al. 2004.

Posselt, Siegfried G. (1986): Budgetkontrolle als Instrument zur Unternehmungssteuerung, Stuttgart 1986.

Pütter, Christiane (2008): Gartners Hype um die Zukunft der AnwendungsEntwicklung, http://www.cio.de/knowledgecenter/soa/857401, 2008, abgerufen am 23.06.2009.

Raimann, Jörg; Mähler, Lutz (2006): Business Intelligence zur Unterstützung des Instandhaltungs-Controllings, in: Controlling, 18. Jg., Heft 1, 2006, S. 39-45.

Ramin, Kurt P.; Kesselmeyer, Bodo; Ott, Stefan (2006a): XBRL im Internal Financial Reporting von Unternehmensgruppen, in: Zeitschrift für internationale und kapitalmarktorientierte Rechnungslegung (KoR), 6. Jg., Heft 3, 2006, S. 179-191.

Ramin, Kurt P.; Kesselmeyer, Bodo; Ott, Stefan (2006b): XBRL im Internal Financial Reporting von Unternehmensgruppen - Neue Möglichkeiten für die Standardisierung und Integration von Finanzprozessen und IT-Systemen (Langfassung), http://anubo.com/fileadmin/user_upload/Fachartikel/XBRL Internal_Financial_Reporting_KRSOBK_060301_Lang.pdf, 2006, abgerufen am 23.06.2009.

Rebstock; Michael; Lipp, Michael (2003): Webservices zur Integration interaktiver elektronischer Verhandlungen in elektronische Marktplätze, in: Wirtschaftsinformatik, 45. Jg., Heft 3, 2003, S. 293-306.

Rechkemmer, Kuno (1998): Qualitatives Rechnungswesen: Grundzüge aus Sicht der Wirtschaftsinformatik, in: Wirtschaftsinformatik, 40. Jg., Heft 5, 1998, S. 380-385. 
Reinefeld, Alexander; Schintke, Florian (2004): Grid Services: Web Services zur Nutzung verteilter Ressourcen, in: Informatik-Spektrum, 27. Jg., Heft 4, 2004, S. 129-135.

Reinheimer, Stefan; Lang, Florian; Purucker, Jörg; Brügmann, Hinnerk (2007): 10 Antworten zu SOA, in: HMD - Praxis der Wirtschaftsinformatik, 44. Jg., Heft 253, 2007, S. 7-17.

Richter, Jan-Peter; Haller, Harald; Schrey, Peter (2005): Serviceorientierte Architektur, in: Informatik-Spektrum, 28. Jg., Heft 5, 2005, S. 413-416.

Ridder, Christopher; Bommer, Kay (2006): Investor Relationship Management - Ein Ansatz für die systematische Kapitalmarktorientierung in der Investor Relationship-Arbeit, in: Finanz Betrieb, 8. Jg., Heft 10, 2006, S. 614621.

Rieks, Marcus (2006): Serviceorientierte Architekturen - Architekturparadigma für die Integration von IT-Systemen, in: Information Management \& Consulting, 21. Jg., Heft 1, 2006, S. 6-10.

Rob, Peter; Coronel, Carlos (2007): Database Systems: Design, Implementation, and Management, Boston, Massachusetts 2007.

Ross, Stephen A.; Westerfield, Randolph W.; Jaffe, Jeffrey (1999): Corporate Finance, Singapur 1999.

Roth, Roman (2007): Wertschöpfungsorientierter Serviceentwurf, in: Starke, Gernot; Tilkov, Stefan (Hrsg.): SOA-Expertenwissen: Methoden, Konzepte und Praxis serviceorientierter Architekturen, Heidelberg 2007, S. 87-110.

Sambamurthy, Vallabh; Bharadwaj, Anandhi; Grover, Varun (2003): Shaping Agility through Digital Options: Reconceptualizing the role of IT in Contemporary Firms, in: MIS Quarterly, 27. Jg., Heft 2, 2003, S. 237-263.

Samtleben, Michael; Stadlbauer, Florian; Hess, Thomas (2006): Anwendungssystemintegration im Controlling: Atueller Stand und wichtige Trends, in: ZfCM - Die Zeitschrift für Controlling \& Management, 50. Jg., Heft 2, 2006, S. 86-93.

Scheer, August-Wilhelm; Bungert, Winfried (2004): Intelligentes Business, in: technologie \& management, o. Jg., Heft 3-4, 2004, S. 12-14.

Scheer, August-Wilhelm; Thomas, Oliver (2005): Geschäftsprozessmodellierung mit der ereignisgesteuerten Prozesskette, in: Das Wirtschaftsstudium (Wisu), 34. Jg., Heft 8-9, 2005, S. 1069-1078. 
Schelp, Joachim (2007): Ansatzpunkte zur Übertragung Serviceorientierter Konzepte auf das Data Warehousing, in: Gluchowski, Peter; Chamoni, Peter; Gersch, Martin; Krebs, Stefan; Reinersmann, Martina (Hrsg.): Schlaglichter der Wirtschaftsinformatik - Festschrift zum 60. Geburtstag von Roland Gabriel, Chemnitz 2007, S. 143-156.

Schelp, Joachim; Aier, Stephan (2008): Serviceorientierte Architekturen - Potentiale für eine nachhaltige Steigerung der Unternehmensagilität, in: Dinter, Barbara; Winter, Robert; Chamoni, Peter; Gronau, Norbert; Turowski, Klaus (Hrsg.): Synergien durch Integration und Informationslogistik, Bonn 2008, S. 529-542.

Schelp, Joachim; Hafner, Martin; Winter, Robert (2006): Organisation des Architekturmanagements, in: Reussner, Ralf; Hasselbring, Wilhelm (Hrsg.): Handbuch der Software-Architektur, Heidelberg 2006, S. 211-227.

Schelp, Joachim; Stutz, Matthias (2007): SOA-Governance, in: HMD - Praxis der Wirtschaftsinformatik, 44. Jg., Heft 253, 2007, S. 66-73.

Schelp, Joachim; Winter, Robert (2007): Agilität und SOA, in: Starke, Gernot; Tilkov, Stefan (Hrsg.): SOA-Expertenwissen: Methoden, Konzepte und Praxis serviceorientierter Architekturen, Heidelberg 2007, S. 41-47.

Scherm, Ewald; Pietsch, Gotthard (2008): Management- und Entscheidungsunterstützung in Organisationen: zwischen Technik und Interaktion, in: Bortfeldt, Andreas; Homberger, Jörg; Kopfer, Herbert; Pankratz, Giselher; Strangmeier, Reinhard (Hrsg.): Intelligent Decision Support - Current Challenges and Approaches: Intelligente Entscheidungsunterstützung - Aktuelle Herausforderungen und Lösungsansätze, Wiesbaden 2008, S. 429-446.

Schinzer, Heiko; Thome, Rainer (1999): Extensible Markup Language, in: Das Wirtschaftsstudium (Wisu), 28. Jg., Heft 2, 1999, S. 208-215.

Schmelz, Jürgen (1995): Business Objects: Schaufenster zum Data Warehouse, in: IT-Management, o. Jg., Heft 3/4, 1995, S. 72-74.

Schmidt, Herrad; Agbor, Ekema (2008): Business Performance Management, in: Das Wirtschaftsstudium (Wisu), 37. Jg., Heft 3, 2008, S. 357-363.

Schmitz, Ulrich (2008): Implementierung von BI: Keine Wunderwaffe ohne solides Fundament, in: BI Spektrum, 3. Jg., TDWI-Sonderheft, 2008, S. 12.

Scholtis, Thomas (1998): Vertragsgestaltung bei Informationsasymmetrie, Wiesbaden 1998.

Schöning, Harald (2003): XML und Datenbanken: Konzepte und Systeme, München/Wien 2003. 
Schröpfer, Christian; Schönherr, Marten (2008): Ein Framework zur Einführung einer SOA-Governance in Unternehmen, in: Dinter, Barbara; Winter, Robert; Chamoni, Peter; Gronau, Norbert; Turowski, Klaus (Hrsg.): Synergien durch Integration und Informationslogistik, Bonn 2008, S. 407-424.

Schruff, Lothar; Kayser, Marc (2002): Das Internet als Medium zur Publizität von Jahres- und Konzernabschlüssen, in: Gabriel, Roland; Hoppe, Uwe (Hrsg.): Electronic Business: Theoretische Aspekte und Anwendungen in der betrieblichen Praxis, Heidelberg 2002, S. 347-359.

Schwalm, Stephan; Bange, Carsten (2004): Einsatzpotenziale von XML in Business-Intelligence-Systemen, in: Wirtschaftsinformatik, 46. Jg., Heft 1, 2004, S. 5-14.

Schwarz, Holger; Kraft, Tobias; Rantzau, Ralf; Mitschang, Bernhard (2003): Optimierung von Anfragesequenzen in Business-IntelligenceAnwendungen, in: it - Information Technology, 45. Jg., Heft 4, 2003, S. 196202.

Schwenk, Jörg (2005): Sicherheit und Kryptographie im Internet: Von sicherer E-Mail bis zu IP-Verschlüsselung, Wiesbaden 2005.

Seidlmeier, Heinrich; Scherfler, Gabriele (2007): Modellgetriebene Integration und Migration - vom Fachprozess zur ausführbaren Anwendung, in: HMD - Praxis der Wirtschaftsinformatik, 44. Jg., Heft 253, 2007, S. 92-104.

Seufert, Andreas; Schiefer, Josef (2008): Business Intelligence als Enabler eines wertorientierten Controllings für das Financial Supply Chain Management, in: ZfCM - Die Zeitschrift für Controlling \& Management, 52. Jg., Sonderheft 2, 2008, S. 16-27.

Siebenhaar, Melanie; Lehrig, Tim; Braun, Johannes; Görge, Thomas (2008): Entwicklung einer SOA-basierten Webanwendung zur Buchung und Verwaltung von Segeltouren: Proprietäre Software vs. Open Source, in: Wirtschaftsinformatik, 50. Jg., Heft 4, 2008, S. 325-329.

Siegwart, Hans (2002): Kennzahlen für die Unternehmungsführung, Bern et al. 2002.

Sommerville, Ian (2001): Software Engineering, München 2001.

Sorter, George H. (1969): An "Events" Approach to Basic Accounting Theory, in: The Accounting Review, 44. Jg., Heft 1, 1969, S. 12-19.

Specht, Thomas; Weisbecker, Anette; Spath, Dieter (2006): Integration Engineering-Methode: Realisierung kooperativer Geschäftsprozesse mit Service-orientierten Architekturen, in: Information Management \& Consulting, 21. Jg., Heft 1, 2006, S. 11-18. 
Tilkov, Stefan; Starke, Gernot (2007): Einmaleins der serviceorientierten Architekturen, in: Starke, Gernot; Tilkov, Stefan (Hrsg.): SOA-Expertenwissen: Methoden, Konzepte und Praxis serviceorientierter Architekturen, Heidelberg 2007, S. 9-36.

Stiemerling, Oliver (2002): Web-Services als Basis für evolvierbare Softwaresysteme, in: Wirtschaftsinformatik, 44. Jg., Heft 5, 2002, S. 435-445.

Stock, Steffen; Büttner, Andreas (2004): Datenqualität in Data-WarehouseSystemen, in: Das Wirtschaftsstudium (Wisu), 33. Jg., Heft 8-9, 2004, S. 1064-1070.

Stock, Steffen; Düsing, Roland (2003): Betriebswirtschaftliche Anforderungen an Data-Warehouse-Systeme, in: it - Information Technology, 45. Jg., Heft 4, 2003, S. 186-189.

Strangmeier, Reinhard (2008): Was ist Wirtschaftsinformatik? - Zum Selbstverständnis einer jungen Wissenschaft, in: Bortfeldt, Andreas; Homberger, Jörg; Kopfer, Herbert; Pankratz, Giselher; Strangmeier, Reinhard (Hrsg.): Intelligent Decision Support - Current Challenges and Approaches: Intelligente Entscheidungsunterstützung - Aktuelle Herausforderungen und Lösungsansätze, Wiesbaden 2008, S. 447-456.

Streibich, Karl-Heinz (2008): Der Paradigmenwechsel ist in vollem Gange, in: Wirtschaftsinformatik, 50. Jg., Heft 1, 2008, S. 73-74.

Strunz, Horst (1990): Informations- und Kommunikationssysteme, München/ Wien 1990.

Klostermeier, Johannes (2008): Credit Suisse setzt 2.200 Web-Services ein, http://www.cio.de/subnet/oracle/praxis-und-diskussion/858941, 2008, abgerufen am 23.06.2009.

Sunyaev, Ali; Leimeister, Jan M.; Schweiger, Andreas; Krcmar, Helmut (2006): Integrationsarchitekturen für das Krankenhaus - Status quo und Zukunftsperspektive, in: Information Management \& Consulting, 21. Jg., Heft 1, 2006, S. 28-35.

Talarczyk, Harald (2008): Excel im Einsatz für BI: An Gewohntes ankoppeln, in: BI Spektrum, 3. Jg., Heft 1, 2008, S. 36.

Terzidis, Orestis; Sure, York; Brelage, Christian (2008): SOA - Flexibility and Agility, in: Wirtschaftsinformatik, 50. Jg., Heft 1, 2008, S. 76.

Theling, Thomas; Janssen, Andre; Loos, Peter (2006): Die Unterstützung von Ausschreibungs- und Vergabeprozessen durch Web Services, in: Schelp, Joachim; Winter, Robert; Frank, Ulrich; Rieger, Bodo; Turowski, Klaus (Hrsg.): Integration, Informationslogistik und Architektur, Bonn 2006, S. 403-426. 
Thomas, Oliver; Leyking, Katrina; Dreifus, Florian (2007): Prozessmodellierung im Kontext serviceorientierter Architekturen, in: HMD - Praxis der Wirtschaftsinformatik, 44. Jg., Heft 253, 2007, S. 37-46.

Thome, Rainer (1998): Informationsverarbeitung als Basis einer neuen zwischenbetrieblichen Firmenkultur, in: Das Wirtschaftsstudium (Wisu), 27. Jg., Heft 8-9, 1998, S. 964-970.

Thome, Rainer (2007): Integration der Informationsverarbeitung, in: Das Wirtschaftsstudium (Wisu), 36. Jg., Heft 5, 2007, S. 655-658.

Thome, Rainer; Böhn, Martin; Hagn, Alexander (2003): DokumentenManagement-Systeme, in: Das Wirtschaftsstudium (Wisu), 32. Jg., Heft 7, 2003, S. 914-922.

Tiobe (2009): TIOBE Programming Community Index for June 2009, http://www.tiobe.com/index.php/content/paperinfo/tpci/index.html, abgerufen am 23.06.2009.

Vielmeyer, Uwe (2004): Risikoorientierte Unternehmenspublizität, Frankfurt am Main et al. 2004.

Vollmuth, Hilmar; Zwettler, Robert (2006): Kennzahlen, Freiburg 2006.

Volnhals, Martina; Hirsch, Bernhard (2008): Information Overload und Controlling, in: ZfCM - Die Zeitschrift für Controlling \& Management, 52. Jg., Sonderheft 1, 2008, S. 50-56.

vom Brocke, Jan (2007): Wirtschaftlichkeit serviceorientierter Architekturen Management und Controlling von Prozessen als Serviceportfolios, in: HMD - Praxis der Wirtschaftsinformatik, 44. Jg., Heft 253, 2007, S. 84-94.

vom Brocke, Jan (2008): Serviceorientierte Architekturen - SOA: Management und Controlling von Geschäftsprozessen, München 2008.

vom Brocke, Jan; Buddendick, Christian (2004): Organisationsformen in der Referenzmodellierung: Forschungsbedarf und Gestaltungsempfehlungen auf Basis der Transaktionskostentheorie, in: Wirtschaftsinformatik, 46. Jg., Heft 5, 2004, S. 341-352.

von Henning, Hans-Jürgen (2007): Granularität von Services: Kriterien zur Strukturierung einer Servicelandschaft, in: Starke, Gernot; Tilkov, Stefan (Hrsg.): SOA-Expertenwissen: Methoden, Konzepte und Praxis serviceorientierter Architekturen, Heidelberg 2007, S. 343-356.

W3C (2009): World Wide Web Consortium, http://www.w3.org, abgerufen am 23.06.2009. 
Wagenhofer, Alfred (2003): Economic Consequences of Internet Financial Reporting, in: Schmalenbach Business Review, 55. Jg., Heft 4, 2003, S. 262279.

Wall, Friederike (2008): Die IT als Enabler guter Entscheidungen: Potenziale und verhaltensbedingte Risiken aus Sicht des Controllings, in: $\mathrm{ZfCM}-\mathrm{Die}$ Zeitschrift für Controlling \& Management, 52. Jg., Sonderheft 1, 2008, S. 8288.

Weber, Jürgen; Bramsemann, Urs; Heineke, Carsten; Hirsch, Bernhard (2002): Value Based Management erfolgreich umsetzen, Weinheim 2002.

Weber, Jürgen; Bramsemann, Urs; Heineke, Carsten; Hirsch, Bernhard (2004): Wertorientierte Unternehmenssteuerung: Konzepte - Implementierung - Praxisstatements, Wiesbaden 2004.

Wenzke, Frank (2005): Business Intelligence, in: Das Wirtschaftsstudium (Wisu), 34. Jg., Heft 4, 2005, S. 631-634.

Werner, Hartmut (2002): Supply Chain Management: Grundlagen, Strategien, Instrumente und Controlling, Wiesbaden 2002.

Werners, Brigitte (2008): Grundlagen des Operations Research, Berlin/ Heidelberg 2008.

Werners, Brigitte (1994): Approximative Inferenz mit linguistischen Variablen, in: Werners, Brigitte; Gabriel, Roland (Hrsg.): Operations Research Reflexionen aus Theorie und Praxis, Festschrift zum 60. Geburtstag von Hans-Jürgen Zimmermann, Berlin et al. 1994, S. 243-274.

Willis, Mike (2003): Corporate Reporting Enters the Information Age, in: Regulation, 26. Jg., Heft 3, 2003, S. 56-60.

Willis, Mike; Hannon, Neal (2005): Combating Everyday Data Problems with XBRL, in: Strategic Finance, 86. Jg., Heft 1, 2005, S. 57-59.

Wilms, Hartmut (2007): WCF als Basis einer SOA, in: Starke, Gernot; Tilkov, Stefan (Hrsg.): SOA-Expertenwissen: Methoden, Konzepte und Praxis serviceorientierter Architekturen, Heidelberg 2007, S. 551-573.

Winkler, Veronika (2007): Identifikation und Gestaltung von Services - Vorgehen und beispielhafte Anwendung im Finanzdienstleistungsbereich, in: Wirtschaftsinformatik, 49. Jg., Heft 4, 2007, S. 257-266.

Workflow Management Coalition (2009): Reference Model, http://www. wfmc.org/reference-model.html, abgerufen am 23.06.2009.

XBRL International (2009): XBRL International, http://www.xbrl.org/Home, abgerufen am 23.06.2009. 
Xiao, Zezhong; Jones, Michael J.; Lymer, Andy (2002): Immediate trends in Internet Reporting, in: The European Accounting Review, 11. Jg., Heft 2, 2002, S. 245-275.

Yusuf, Yahaya Y.; Sarhadi, Mansoor; Gunasekaran, Angappa (1999): Agile manufacturing: The drivers, concepts and attributes, in: International Journal of Production Economics, 62. Jg., Heft 1-2, 1999, S. 33-43.

Zdrowomyslaw, Norbert; Kasch, Robert (2002): Betriebsvergleiche und Benchmarking für die Managementpraxis, München 2002. 
Alexander Pastwa - 978-3-631-75488-7

Downloaded from PubFactory at 01/11/2019 04:20:29AM

via free access 


\section{Bochumer Beiträge zur Unternehmensführung}

Herausgegeben vom Direktorium des Instituts

für Unternehmensführung der Ruhr-Universităt Bochum

Band 1 Busse von Colbe, Walther/Mattessich, Richard (Hrsg.): Der Computer im Dienste der Unternehmungsführung (1968)

Band 2 Busse von Colbe, Walther/Meyer-Dohm, Peter (Hrsg.): Unternehmerische Planung und Entscheidung (1969)

Band 3 Anthony, Robert N.: Harvard-Fälle aus der Praxis des betrieblichen Rechnungswesens. Herausgegeben von Richard V. Mattessich unter Mitarbeit von Klaus Hermberger und Wolf Lange (1969)

Band 4 Mattessich, Richard: Die wissenschaftlichen Grundlagen des Rechnungswesens (1970)

Band 5 Schweim, Joachim: Integrierte Unternehmungsplanung (1969)

Band 6 Busse von Colbe, Walther (Hrsg.): Das Rechnungswesen als Instrument der Unternehmungsführung (1969)

Band 7 Domsch, Michel: Simultane Personal- und Investitionsplanung im Produktionsbereich (1970)

Band 8 Leunig, Manfred: Die Bilanzierung von Beteiligungen. Eine bilanztheoretische Untersuchung (1970)

Band 9 Franke, Reimund: Betriebsmodelle. Rechensystem für Zwecke der kurzfristigen Planung, Kontrolle und Kalkulation (1972)

Band 10 Wittenbrink, Hartwig: Kurzfristige Erfolgsplanung und Erfolgskontrolle mit Betriebsmodellen (1975)

Band 11 Lutter, Marcus (Hrsg.): Recht und Steuer der internationalen Unternehmensverbindungen (1972)

Band 12 Niebling, Helmut: Kurzfristige Finanzrechnung auf der Grundlage von Kosten- und Erlosmodellen (1973)

Band 13 Perlitz, Manfred: Die Prognose des Unternehmenswachstums aus Jahresabschlüssen deutscher Aktiengesellschaften (1973)

Band 14 Niggemann, Walter: Optimale Informationsprozesse in betriebswirtschaftlichen Entscheidungssituationen (1973)

Band 15 Richardt, Harald: Der aktienrechtliche Abhăngigkeitsbericht unter okonomischen Aspekten (1974)

Band 16 Backhaus, Klaus: Direktvertrieb in der Investitionsgüterindustrie - Eine Marketing-Entscheiung (1974)

Band 17 Plinke, Wulff: Kapitalsteuerung in Filialbanken (1975)

Band 18 Steffen, Rainer: Produktionsplanung bei Fließbandfertigung (1977)

Band 19 Kolb, Jürgen: Industrielle Erlosrechnung - Grundlagen und Anwendungen (1978)

Band 20 Busse von Colbe, Walther/Lutter, Marcus (Hrsg.): Wirtschaftsprüfung heute: Entwicklung oder Reform? (1977)

Band 21 Uphues, Peter: Unternehmerische Anpassung in der Rezession (1979)

Band 22 Gebhardt, Günther: Insolvenzprognosen aus aktienrechtlichen Jahresabschlüssen (1980)

Band 23 Domsch, Michel: Systemgestützte Personalarbeit (1980) 
Band 24 Schmied, Volker: Alternativen der Arbeitsgestaltung und ihre Bewertung (1982)

Band 25 Wäscher, Gerhard: Innerbetriebliche Standortplanung bei einfacher und mehrfacher Zielsetzung (1982)

Band 26 Weber, Martin: Entscheidungen bei Mehrfachzielen - Verfahren zur Unterstützung von Individual- und Gruppenentscheidungen (1983)

Band 27 Kroesen, Alfred: Instandhaltungsplanung und Betriebsplankostenrechnung (1983)

Band 28 Plinke, Wulf: Erlösplanung im industriellen Anlagengeschäft (1985)

Band 29 Chamoni, Peter: Simulation störanfälliger Systeme (1986)

Band 30 Arning, Andreas: Die wirtschaftliche Bewertung der Zentrenfertigung - Dargestellt am Beispiel einer Fertigungsinsel (1987)

Band 31 Gebhardt, Günther: Finanzielle Planung und Kontrolle bei internationaler Unternehmenstätigkeit

Band 32 Markiewicz, Michael: Ersatzteildisposition im Maschinenbau - Betriebswirtschaftliche Methoden der Planung und Überwachung (1988)

Band 33 Pellens, Bernd: Der Informationswert von Konzernabschlüssen - Eine empirische Untersuchung deutscher Börsengesellschaften (1989)

Band 34 Mrotzek, Rüdiger: Bewertung direkter Auslandsinvestitionen mit Hilfe betrieblicher Investitionskalküle (1989)

Band 35 Deppe, Joachim: Quality Circle und Lernstatt - Ein integrativer Ansatz (1989, 3. Auflage 1993)

Band 36 Rademacher, Michael: Arbeitszeitverkürzung und -flexibilisierung - Formen und betriebliche Auswirkungen (1990)

Band 37 Kaiser, Klaus: Kosten- und Leistungsrechung bei automatisierter Produktion (1991, 2. Auflage 1993)

Band 38 Müller, Hermann: Industrielle Abfallbewältigung - Entscheidungsprobleme aus betriebswirtschaftlicher Sicht (1991)

Band 39 Schörner, Peter: Gesetzliches Insiderhandelsverbot - Eine ordnungspolitische Analyse (1991)

Band 40 Bentler, Martin: Grundsătze ordnungsmäßiger Bilanzierung für die Equitymethode (1991)

Band 41 Brüggerhoff, Jürgen: Management von Desinvestitionen (1992)

Band 42 Bröker, Erich W.: Erfolgsrechnung im industriellen Anlagengeschäft - Ein dynamischer Ansatz auf Zahlungsbasis - (1993)

Band 43 Frankenberg, Peter: Transnationale Analyse US-amerikanischer und deutscher Jahresabschlüsse - Eine theoretische und empirische Untersuchung (1993)

Band 44 Kleinaltenkamp, Michael: Standardisierung und Marktprozeß - Entwicklungen und Auswirkungen im CIM-Bereich (1993)

Band 45 Pellens, Bernhard: Aktionärsschutz im Konzern - Empirische und theoretische Analyse der Reformvorschläge der Konzernverfassung (1994)

Band 46 Reckenfelderbăumer, Martin: Marketing-Accounting im Dienstleistungsbereich - Konzeption eines prozeßkostengestützten Instrumentariums (1995)

Band 47 Knittel, Friedrich: Technikgestützte Kommunikation und Kooperation im Büro. Entwicklungshindernisse - Einsatzstrategien - Gestaltungskonzepte (1995)

Band 48 Riezler, Stephan: Lebenszyklusrechnung - Instrument des Controlling strategischer Projekte (1996) 
Band 49 Schulte, Jörn: Rechnungslegung und Aktienkursentwicklung - Erklärung und Prognose von Aktienrenditen durch Einzel- und Konzernabschlußdaten (1996)

Band 50 Muhr, Martin: Zeitsparmodelle in der Industrie - Grundlagen und betriebswirtschaftliche Bedeutung mehrjähriger Arbeitszeitkonten (1996)

Band 51 Brotte, Jörg: US-amerikanische und deutsche Geschäftsberichte. Notwendigkeit, Regulierung und Praxis jahresabschlußergänzender Informationen (1997)

Band 52 Gersch, Martin: Vernetzte Geschäftsbeziehungen. Die Nutzung von EDI als Instrument des Geschäftsbeziehungsmanagement (1998)

Band 53 Währisch, Michael: Kostenrechnungspraxis in der deutschen Industrie. Eine empirische Studie (1998)

Band 54 Völkner, Peer: Modellbasierte Planung von Geschäftsprozeßabläufen (1998)

Band 55 Fülbier, Rolf Uwe: Regulierung der Ad-hoc-Publizităt. Ein Beitrag zur okonomischen Analyse des Rechts (1998)

\section{Band 1 - 55 erschienen beim Gabler Verlag Wiesbaden}

Band 56 Ane-Kristin Reif-Mosel: Computergestützte Kooperation im Büro. Gestaltung unter Berücksichtigung der Elemente Aufgabe, Struktur, Technik und Personal (2000)

Band 57 Claude Tomaszewski: Bewertung strategischer Flexibilităt beim Unternehmenserwerb. Der Wertbeitrag von Realoptionen (2000)

Band 58 Thomas Erler: Business Objects als Gestaltungskonzept strategischer Informationssystemplanung (2000)

Band 59 Joachim Gassen: Datenbankgestützte Rechnungslegungspublizităt. Ein Beitrag zur Evolution der Rechnungslegung (2000)

Band 60 Frauke Streubel: Organisatorische Gestaltung und Informationsmanagement in der lernenden Unternehmung. Bausteine eines Managementkonzeptes organisationalen Lernens (2000)

Band 61 Andreas von der Gathen: Marken in Jahresabschluß und Lagebericht (2001)

Band 62 Lars Otterpohl: Koordination in nichtlinearen dynamischen Systemen (2002)

Band 63 Ralf Schremper: Aktienrückkauf und Kapitalmarkt. Eine theoretische und empirische Analyse deutscher Aktienrückkaufprogramme (2002)

Band 64 Peter Ruhwedel: Aufsichtsratsplanungssysteme. Theoretische Grundlagen und praktische Ausgestaltung in Publikumsaktiengesellschaften (2002)

Band 65 Jens Thorn: Taktisches Supply Chain Planning. Planungsunterstützung durch deterministische und stochastische Optimierungsmodelle (2002)

Band 66 Dirk Beier: Informationsmanagement aus Sicht der Betriebswirtschaftslehre. Theoretische Ansätze und das Beispiel Mobile Business. (2002)

Band 67 Nils Crasselt: Wertorientierte Managemententlohnung, Unternehmensrechnung und Investitionssteuerung. Analyse unter Berücksichtigung von Realoptionen. (2003)

Band 68 Franca Ruhwedel: Eigentümerstruktur und Unternehmenserfolg. Eine theoretische und empirische Analyse deutscher börsennotierter Unternehmen. (2003)

Band 69 Andreas Bonse: Informationsgehalt von Konzernabschlüssen nach HGB, IAS und USGAAP. Eine empirische Analyse aus Sicht der Eigenkapitalgeber. (2004) 
Band 70 Thorsten Sellhorn: Goodwill Impairment. An Empirical Investigation of Write-Offs under SFAS 142. (2004)

Band 71 Bernd Slaghuis: Vertragsmanagement für Investitionsprojekte. Quantitative Projektplanung zur Unterstützung des Contract Managements unter Berücksichtigung von Informationsasymmetrie. (2005)

Band 72 Stephanie Freiwald: Supply Chain Design. Robuste Planung mit differenzierter Auswahl der Zulieferer. (2005)

Band 73 Rolf Uwe Fülbier: Konzernbesteuerung nach IFRS. IFRS-Konsolidierungsregeln als Ausgangspunkt einer konsolidierten steuerlichen Gewinnermittlung in der EU? (2006)

Band 74 Marc Richard: Kapitalschutz der Aktiengesellschaft. Eine rechtsvergleichende und ökonomische Analyse deutscher und US-amerikanischer Kapitalschutzsysteme. (2007)

Band 75 Sonja Schade: Kennzahlengestütztes Controlling für mittelständische Unternehmenskooperationen. (2007)

Band 76 Peter Weber: Analyse von Lern-Service-Geschäftsmodellen vor dem Hintergrund eines sich transformierenden Bildungswesens. (2008)

Band 77 Karsten Detert: Bilanzpolitisches Verhalten bei der Umstellung der Rechnungslegung von HGB auf IFRS. Eine empirische Untersuchung deutscher Unternehmen. (2008)

Band 78 Stefan Neuhaus: Auslagerung betrieblicher Pensionszusagen. Eine ökonomische Analyse der Motive und Durchführungsformen. (2009)

Band 79 Alexander Pastwa: Serviceorientierung im betrieblichen Berichtswesen. Entwicklung eines Architektur- und Vorgehensmodells zur konzeptionellen Gestaltung von Berichtsprozessen auf Basis einer SOA und XBRL. (2010)

uww.peterlang.de 